\title{
The evolution of beliefs and strategic behavior
}

Citation for published version (APA):

Opolot, D. C. (2015). The evolution of beliefs and strategic behavior. [Doctoral Thesis, Maastricht University]. Maastricht University. https://doi.org/10.26481/dis.20150603do

Document status and date:

Published: 01/01/2015

DOI:

10.26481/dis.20150603do

Document Version:

Publisher's PDF, also known as Version of record

\section{Please check the document version of this publication:}

- A submitted manuscript is the version of the article upon submission and before peer-review. There can be important differences between the submitted version and the official published version of record.

People interested in the research are advised to contact the author for the final version of the publication, or visit the DOI to the publisher's website.

- The final author version and the galley proof are versions of the publication after peer review.

- The final published version features the final layout of the paper including the volume, issue and page numbers.

Link to publication

\footnotetext{
General rights rights.

- You may freely distribute the URL identifying the publication in the public portal. please follow below link for the End User Agreement:

www.umlib.nl/taverne-license

Take down policy

If you believe that this document breaches copyright please contact us at:

repository@maastrichtuniversity.nl

providing details and we will investigate your claim.
}

Copyright and moral rights for the publications made accessible in the public portal are retained by the authors and/or other copyright owners and it is a condition of accessing publications that users recognise and abide by the legal requirements associated with these

- Users may download and print one copy of any publication from the public portal for the purpose of private study or research.

- You may not further distribute the material or use it for any profit-making activity or commercial gain

If the publication is distributed under the terms of Article $25 \mathrm{fa}$ of the Dutch Copyright Act, indicated by the "Taverne" license above, 


\section{The evolution of beliefs and strategic behavior}

Daniel Opolot

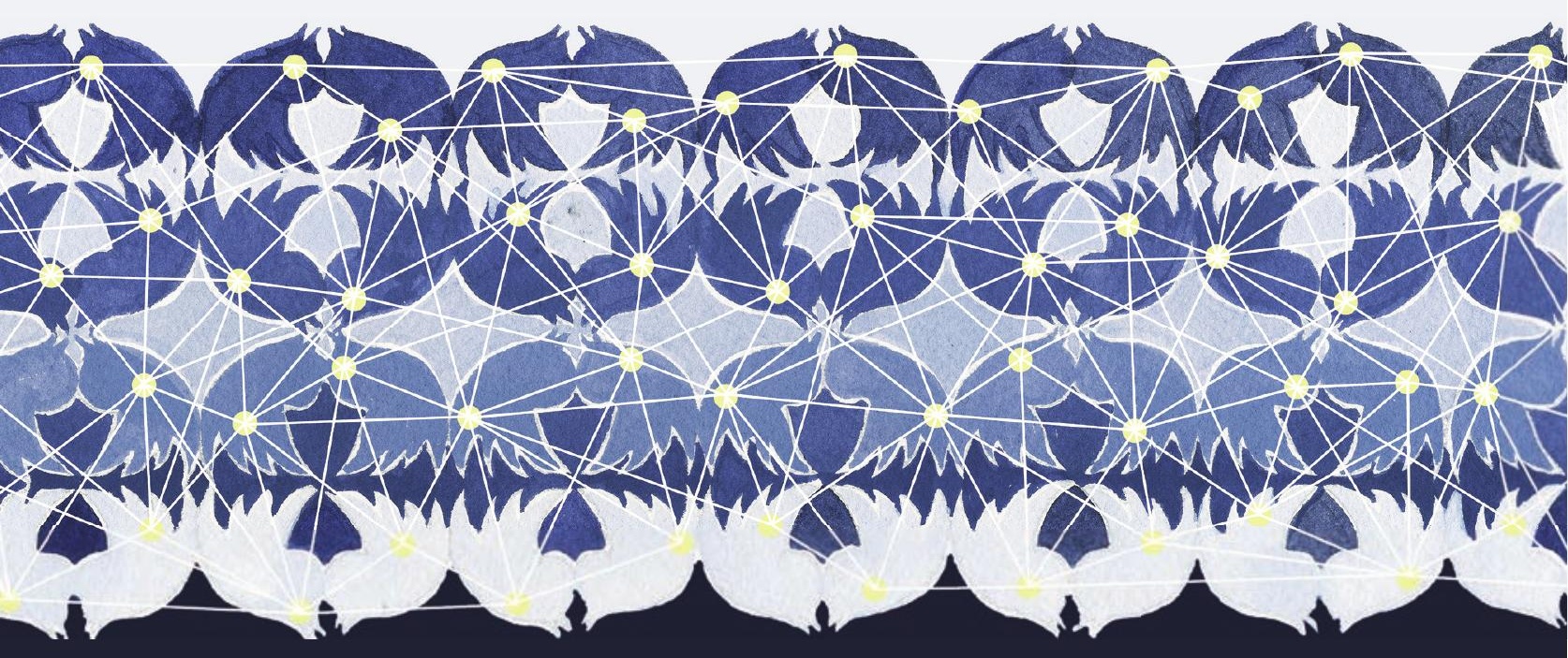


The front page image is a painting created by Vanessa Hodgkinson, and was inspired by the content of this dissertation.

http://www.vanessahodgkinson.com/ 
THE EVOLUTION OF BELIEFS AND STRATEGIC BEHAVIOR

\section{DISSERTATION}

to obtain the degree of Doctor at Maastricht University, on the authority of the Rector Magnificus, Prof. dr. L. L. G.

Soete, in accordance with the decision of the Board of Deans, to be defended in public on

Wednesday 03 June 2015, at 12.00 hours

by

Daniel Christopher Opolot 


\section{Supervisor}

Prof. dr. Théophile T. Azomahou

\section{Assessment Committee}

Prof. dr. Robin Cowan (Chair)

Prof. dr. Vianney Dequiedt (Universite d'Auvergne)

Prof. dr. Jean-Jacques Herings

Prof. dr. Alan Kirman (Aix-Marseille Universite, EHESS and IUF)

Associate Prof. dr. Arkadi Predtetchinski 
This dissertation theoretically studies how evolutionary game theory and observational learning can be used to model three aspects of economic and social interactions: repeated interactions, experimentation and communication. All these three aspects influence individual beliefs and behavior and in turn social welfare. Unlike classical game theory, learning and evolutionary game theory relaxes the assumptions of perfect rationality and foresight making it suitable for modeling such interaction environments. The organization of the dissertation is into two themes. The first part focuses on the aspects of repeated interactions and experimentation when agents behave strategically, and the second part focuses on evolution of individual beliefs under repeated interactions and communication.

Chapter 2 analyzes aggregate outcomes of stochastic evolutionary processes, that is repeated strategic interactions with experimentation. It develops computational methods for identifying the most likely outcomes in such interactions. In this regard, we define epsilon stability as the appropriate solution concept. That is, epsilon stable states (hence strategies) are the most likely outcomes in the long-run given the level of experimentation. We use this concept to show that if probabilities of mistakes depend on payoff losses associated with the transition, then risk-dominant strategies are the most likely to be played in the long-run. Risk-dominant strategies however need not be epsilon stable in general.

Chapter 3 studies learning and evolutionary processes under limited information. That is players are assumed to observe the behavior of only a select few from the population. These select few are each player's social or ego-network. The Chapter particularly focuses on large decentralized societies, whereby we define asymptotic global stability as an appropriate solution concept. A state and hence strategy is said to be asymptotically globally stable if it is uniquely stable in the long-run at the limit of the population size. Unlike the case of global interactions, limited information ensures that asymptotic global stability is robust to the model of experimentation. We identify global and path-wise contagion as key factors that are compatible with asymptotic global stability. These two factors depend on the relative payoff gains and network structure. The implications are that for any given payoff structure and hence relative payoff gains, it is possible to design a network such that a unique strategy is asymptotically globally stable. Similarly, for a given network structure 
and set of strategies, one can determine the relative payoff gains that lead to a given strategy to be globally stable.

Chapter 4 studies rates of convergence of stochastic evolutionary processes. The main result is that the speed of evolution is influenced by three main factors: the payoff gains, the topology of the interactions among agents and the level of noise. For a given level of noise, there is no clear-cut linear relationship between convergence rates and payoff gains and topology of interactions. The key factor is whether or not long-run epsilon stable strategies are globally contagious. Global contagion leads to shorter convergence time to the epsilon stable states but longer convergence times to stationarity. When global contagion is feasible, the expected waiting time from any other subset of states to the long-run stable set is shorter for highly than sparsely connected networks. The reverse is true for convergence time. Networks for which global contagion is infeasible lead to shorter convergence time.

Chapter 5 studies the evolution of individual beliefs through repeated interactions and word-of-mouth communication. Three main factors are modeled as relevant in determining the structure of beliefs over time: historical factors-prior beliefs, the learning mechanism (the manner in which individuals incorporate new information into their beliefs)-rational or bounded-rational learning, and the topology of communication structure governing information exchange. Heterogeneity in public beliefs resulting from such interactions is more likely when individuals are rational than when they are not. This could result from heterogeneity in historical factors, topology of interaction structure or both. We also examine conditions under which the resulting public beliefs correctly aggregate decentralized private information. Under rational learning, the only relevant factors for correct aggregation is knowledge of the network topology (and not the network topology) and boundedness of private beliefs. The network topology however plays a significant role for correct aggregation under bounded-rational learning 
This dissertation is a final product of the journey that began nearly six years ago when a very good friend, Anne-Marie Nzioki, told me about the PhD program offered by UNU-MERIT. The journey has been long, tedious but equally gratifying. It has taken a lot of support from colleagues, friends and family to arrive at this stage. In this regard, I would like to extend my sincere gratitude and appreciation to the following categories of people.

First and foremost to my supervisor and promoter Théophile Azomahou, you have been extremely supportive in all respects. This project has evolved a lot from what the original proposal had suggested and this would not have been the case if you were not to be patient enough with my ever evolving ideas. You also encouraged me to always go for that one extra step, the benefits of which are not always apparent until the final stage. Thank you very much for this. A special thank you also goes to the reading committee: Robin Cowan, Jean-Jacques Herings, Alan Kirman, Vianney Dequiedt, Arkadi Predtetchinski. Thank you for the time you spent reading this thesis. Your comments and suggestions have been valuable. Chapter 3 has particularly benefited from the comments you provided on the earlier version. The thesis has also benefited a lot from comments and suggestion by the seminar participants at The Centre d'Economie de La Sorbonne and Paris School of Economics, University of Cergy Pontoise THEMA, Cournot seminars at Bureau d'Economie Théorique et Appliquée (BETA), University of Strasbourg, Royal Economic Society Postgraduate Presentation Meeting \& Job Market, 1oth Workshop on Networks in Economics and Sociology at Utrecht School of Economics, and Maastricht Lecture Series in Economics.

The second category of people from whom this thesis has directly benefited from is those that I have discussed it with multiple times for the last four years. Francois Lafond and Giorgio Triulzi, you two have been a huge influence on the direction that this project took. Stefania Innocenti, discussions with you have been influential and not forgetting all the last stage contributions you made towards proof-reading and the final outlook of the book. Samyukta Bhupatiraju, Sheng Zhong, Francesca Guadagno and George Okongo, thank you all for all forms of proof-readings you did.

The third category of people I would like to thank is those that have contributed indirectly to the success of this PhD journey. My parents, your influence in the success of this journey goes way back to my childhood. The principles of hard work and perseverance that you inculcated in me early on could not have come any handier. 
Uncle Igulot H. Patrick, you have done quite a lot in this regard as well. Elham Nikpey, you were a part of my life for most of this journey and I greatly appreciate the level of patience you did exercise. Eveline de Braek, your open-door policy was of great help right from my first day in Maastricht. Wilma Coenegrachts, Marc Vleugels, Eric Engelen, Howard Hudson, Herman Pijpers, Mourik, Ad Notten and Monique Raedts, thank you for all the administrative help you offered throughout.

I am very fortunate to have met a lot of nice and friendly people in Maastricht. Tatevik Poghosyan, Alejandro Lavopa and Jocelyn Olivari, I could not have asked for better batch-mates. The fun moments will forever be in memory. A lot of people made Maastricht feel like home: Jennifer Taborda, Andrea Franco, Charlotte Guillard, Daniel Vertesy, Shuan, Iman, Lilia, Rodolfo, Eduardo, Hibret, Michael, Ajay, Simone, Ilire, Andrés, Andy, Alison (you were a fantastic addition to the office), Elisa, Elvis, Omar, Boris, Jojo, Michiko, Marco, Sachin, Saba, Ibrahima, Mary, Juan Carlos, Ayo, Rachael, Eleni, Dorcas, Maty, Nyasha, Yesuf, Ayla, Fernando, Eva, Tobias, Paula, Thuy, Agustine, Julieta, Irina B, Francesca R, Tigist, Nevena, Gabri and many other Maastrichtians, it would not have been the same without you all.

Finally, I would like to extend my most enthusiastic gratitude to the cover image crew, Charlotte Rooijackers and Vanessa Hodgkinson, you two were last minute saviors in this respect. Vanessa you did a tremendous job to conceptualize and finish up the painting in less than two days. It is a befitting image that completes the story of the thesis.

This project was made possible by the financial support from UNU-MERIT, to which I am grateful for. 


\section{CONTENTS}

1 INTRODUCTION 1

2 EPSILON STABILITY AND ROBUST STOCHASTIC STABILITY 7

2.1 Introduction 7

2.2 Conceptual example 11

2.3 The model 14

2.3.1 Dynamics and learning scheme 14

2.3.2 Stochastic and epsilon stability 16

2.4 Stochastic and epsilon stable sets $\quad 17$

2.5 The mistakes model 22

2.6 Multinomial logit model 24

2.7 Examples 26

2.8 Conclusion 30

2.9 Appendix 31

2.9.1 Proof of Lemma 531

2.9.2 Proof of Proposition $4 \quad 32$

2.9.3 Proof of Theorem $1 \quad 34$

2.9.4 Proof of Proposition 235

3 StOCHAStic EVOLUTION IN NETWORKS 37

3.1 Introduction 37

3.2 The model 42

3.3 Asymptotic global convergence 44

3.3.1 Exit probabilities 45

3.3.2 Contagion probabilities $\quad 46$

3.4 Global contagion 52

3.5 Path-wise contagion 55

3.6 Other examples 60

3.7 Concluding remarks 62

3.8 Appendix 62

3.8.1 Proof of Lemma 562

3.8.2 Proof of Proposition 563

3.8.3 Proofs for Example $6 \quad 66$

3.8.4 Proof of Proposition $7 \quad 67$ 
3.8.5 Proof of Theorem $2 \quad 68$

4 CONVERGENCE RATES FOR STOCHASTIC EVOLUTION IN NETWORKS 71

4.1 Introduction 71

4.2 The model 75

4.2.1 Dynamics and learning scheme $\quad 76$

4.2.2 Closed communication classes and basins of attraction $\quad 77$

4.3 Expected waiting time $\quad 78$

4.3.1 Example 8o

4.4 Convergence time 81

4.5 Deterministic networks 86

4.5.1 $n$-dimensions nearest neighbor interactions $\quad 87$

4.5.2 $n$-dimensions $l$-max distance interactions 90

4.6 Random networks 92

4.7 Step-by-step evolution 95

4.8 Concluding remarks 99

4.9 Appendix 99

4.9.1 Proof of Lemma 1099

4.9.2 Proof of Theorem 3100

4.9.3 Proof of Theorem 4 101

5 BELIEFS DYNAMICS IN COMMUNICATION NETWORKS 105

5.1 Introduction 105

5.1.1 Summary of results 107

5.1.2 Relation to the literature 108

5.2 The model 112

5.2.1 Communication network 113

5.2.2 Dynamic networks 114

5.2.3 Rational learning 114

5.2.4 Bounded-rational learning 115

$5 \cdot 3$ Public beliefs 116

5.3.1 Rational learning 116

5.3.2 Bounded-rational learning 119

5.4 Correct public beliefs 122

5.4.1 Rational learning 123

5.4.2 Bounded rational learning 125

5.4.3 Asymptotically balanced networks 126

5.5 Convergence rate and efficient information aggregation 130

5.5.1 Convergence rate 130 
5.5.2 Efficient information aggregation 131

5.6 Conclusion 134

5.7 Appendix 136

5.7.1 Proof of Theorem $5 \quad 136$

5.7.2 Proof of Proposition $11 \quad 140$

5.7.3 Proof of Theorem $6 \quad 142$

5.7.4 Proof of Theorem $7 \quad 143$

5.7.5 Proof of Proposition $12 \quad 145$

5.7.6 Proof of Theorem $8 \quad 147$

5.7.7 Proof of Proposition $13 \quad 147$

5.7.8 Proof of Proposition $14 \quad 148$

6 VALORIZATION 151 



\section{I}

\section{INTRODUCTION}

Many economic and social environments involve repeated interactions, experimentation and communication among agents (individuals, firms, political parties or countries) over time. The following four examples illustrate such environments. The first is bargaining, which ranges from cross-border negotiations among neighboring countries, to policy negotiations among legislators and expenditure negotiations among household members. The second example is the formation of conventions such as social norms concerning punctuality and social contracts, and adoption of technological innovations. Both of these cases involve an element of repeated interactions and trial and error. The third example is the emergence of cooperation among agents that can be viewed as a result of repeated interactions. Unless if one assumes that the interacting entities posses high levels of rationality and common knowledge, there is no particular reason to assume that a one-short meeting or interaction can directly lead to cooperation. Cooperation requires trust, and trust can only be developed after repeated interactions. Here, we use the word "experimentation" both as an abstraction from the process of trial and error and to model the assumption that agents occasionally make mistakes in their decision processes.

The fourth example is that of belief and preference formation. Individual preferences and beliefs about certain subjects generally evolve over time through observational learning and/or direct communication with others. Beliefs resulting from such interactions play a significant role in determining public opinions and decisions made under uncertainty, both of which in turn shape social welfare. For example the level of heterogeneity in beliefs about government policies such as public health and social integration initiatives affects their implementation. Similarly, beliefs among scientists about the truthfulness of certain scientific theories shape their evolution. Decision making under uncertainty such as agents deciding whether or not to adopt a certain product or practice without complete information of its benefit, is a ubiquitous phenomenon in social and economic interactions. Under such situations individuals update their beliefs after observing others' choices or after communicating 
their private beliefs to each other, and the resulting beliefs determine actions that will be taken. The private belief is what results after an agent incorporates his private information into his prior belief. It is therefore important to study the properties of beliefs resulting from such interactions and whether or not they correctly aggregate private information.

To explicitly model such economic and social situations, it is necessary to capture their dynamic aspect, underlying interaction and incentive structures, as well as the behavioral rules governing individual decision process. What makes such interaction environments even more complicated to model is that they in most cases exhibit strategic complementarity, a property that also implies multiplicity of possible outcomes. A robust theoretical model is therefore that which is able to equally predict the most probable among all possible outcomes. The theory of learning and evolution in games provides the most suitable method in that its flexibility allows for a detailed modeling of repeated interactions, experimentation as well as equilibrium selection. The basic idea behind the theory of learning in games can be analogized as follows. Players (economic agents) interact repeatedly, whereby each player can be viewed as a machine. Each machine possesses a certain memory and an output rule that maps every possible state of memory to a decision. The machine's state evolves over time due to new information learned from others. The set of rules, specified for each player, relating decisions to states of memory and how players incorporate information learned from others then determine the evolution of play.

The theory of learning in games dates back to the work of Brown [24] and Robinson [93] on fictitious play. Under fictitious play, the learning process follows the above analogized steps and players are assumed to possess infinite memory. That is, at each period a player's beliefs about the opponents' behavior are given by the time average of the entire history of play. These beliefs are then used in choosing the best response to opponents' behavior. Brown [24] first introduced fictitious play as an alternative justification for the concept of Nash equilibrium as opposed to the original explanation that relied on assumptions of individual rationality and common knowledge. These two assumptions were viewed as stringent and also later seen to be unrepresentative of real-world economic and social decision processes (e.g. McKelvey and Palfrey [73], Nasar [84]). Brown [24] and Robinson [93] showed that indeed players learn to play Nash equilibrium (in two player finite strategy zero-sum games), confirming Nash's statement that "It is unnecessary to assume that the participants have full knowledge of the total structure of the game, or the ability and inclination to go through any complex reasoning process" (Nash [85, p. 21]). 
Despite the developments of the theory of learning in games in the 1950's and early 6o's, the general interest faded from mid 6o's after Shapley et al. [105] provided a counter example in which play fails to converge. That is cases in which play ends up cycling among a subset of alternatives indefinitely. From the early 1990's however, there has been a revival of the theory. The main reason for this revival is scant evidence in support of the assumptions of individual rationality and common knowledge on one hand (e.g. McKelvey and Palfrey [73], Nasar [84]), and on the other hand the growing empirical evidence for the theory of learning (e.g. Camerer and Ho [25], Cooper et al. [30], Erev and Roth [39], Lettau and Uhlig [69], Roth and Erev [96] and Goeree and Holt [48]). ${ }^{1}$ The current literature can be categorized into two. The first class follows in the tradition of Brown [24] and Robinson [93] and focuses on investigating the foundations of the concept of Nash equilibrium (e.g. Fudenberg and Kreps [44], Fudenberg and Kreps [45], Kalai and Lehrer [56], Hofbauer and Sandholm [50] and Nachbar [83]). Not surprisingly, its analysis centers around identifying game environments in which play converges to Nash equilibria.

The second class of the literature focuses on equilibrium selection (e.g. Foster and Young [41], Young [113] and Kandori et al. [57]). In this class of models, the learning process still proceeds as analogized above but players are instead assumed to posses bounded memory and occasionally experiment on their strategies. The objective is then to identify, for a given underlying game, the most likely states in the long-run at the limit of noise. These long-run stable states are then said to be stochastically stable. As the reader might have already guessed, such learning mechanisms and hence stochastically stable states can be sensitive to the model and level of experimentation or noise. In the case of robustness to the model of experimentation, we can take comfort in studying the outcomes of the learning process for specific families of models provided of course that one can identify models that are appropriate for each interaction environments. In the case of robustness to the level of noise however, it is less comforting in that if for a given model of experimentation the stochastically stable outcomes are not necessarily those that are most likely at moderately small levels of noise, then caution aught to be taken when interpreting the results.

This dissertation makes theoretical and methodological contributions to the theory of learning both in strategic (games) and non-strategic interaction environments. In the case of strategic interaction environments, our approach is in line with the second class of models in that we assume players to posses bounded memory. We

I Since its resurgence, several applications such as bargaining, signaling, reputation formation, cultural integration, contagion and social innovation have been developed. See for example Vega-Redondo [111], Nöldeke and Samuelson [88], Kandori and Rob [58], Kirman and Vriend [6o], Binmore et al. [18], Kuran and Sandholm [65], Alós-Ferrer and Weidenholzer [9], Huck et al. [53]. 
however emphasize the relevance of the process of experimentation in the agents' decision processes. This implies that we have to define an appropriate solution concept, that we refer to as epsilon stability, rather than that of stochastic stability. But since stochastic stability is relatively computationally convenient, our analysis also involves providing conditions under which epsilon stability can be approximated by stochastic stability. The second aspect we emphasize concerns limited information. Not only do agents posses bounded memory, in some interaction environments information can be decentralized. This is particularly the case if interactions are restricted to ego-social networks. Similarly, under such settings stochastic stability is no longer an appropriate solution concept especially if one is interested in large decentralized societies. We then also define an appropriate solution concept for this interaction environment to be asymptotic global stability. That is stability at the limit of population size.

In the case of non-strategic interactions, we maintain the structure of the learning process as analogized above but this time agents revise their beliefs rather than strategies. We also model in more detail the process by which agents incorporate information learned from others. That is, the case where they are assumed to be rational or boundedly rational. In both strategic and non-strategic interaction environments, our analysis involves the study of long-run behavior, computational methods for identifying long-run outcomes and analysis of convergence rates of the processes of learning.

Chapter 2 focuses on computational methods and the refinement of the widely employed solution concept in evolutionary game theory: stochastic stability. An outcome is said to be stochastically stable if the probability with which it is played in the long-run is positive at the limit of noise. The main questions we deal with relate to the relevance of performing limit noise analysis as discussed above. In so doing, we introduce a related concept of stability that we refer to as epsilon stability. Just like stochastically stable outcomes are those which are the "most" stable in the long-run in the limit of noise, epsilon stable outcomes are those that are most stable in the long-run for positive noise levels. We then say that stochastic stability is robust to noise for a given model of mistakes if there exists an equivalence between epsilon and stochastic stability. We provide conditions for robustness for two classes of models of mistakes. The mistakes model in which errors result from pure experimentation, and the endogenous model of mistakes in which errors result from payoff perturbations.

Chapter 2 also provides the computational methods for both epsilon and stochastic stability. The existing computational methods for stochastic stability are specific to the models of mistakes. Here, we provide a more general method that is inde- 
pendent of the model of mistakes. This enables us to derive general results for the characteristics of the stochastically stable outcomes for each category of model of mistakes.

Chapter 3 studies the process of learning under limited information. Limited information results from the assumption that agents interact with only those in their social circle or ego-networks. We particularly focus on large decentralized societies and define asymptotic global stability as an appropriate solution concept. That is, a state and hence strategy is said to be asymptotically globally stable if it is uniquely stable in the long-run at the limit of the population size. We provide an explicit characterization of how the interaction network and underlying base game structures interactively influence asymptotic global stability.

Chapter 4 deals with the question of convergence rates. One of the earlier criticisms of stochastic stability as a solution concept is that at the limit of noise, the expected waiting time to reach the stochastically stable outcome from any other possible equilibrium outcome becomes exceedingly long. This then casts doubts on whether the long-run properties of such models carry any realizable economic implications. In some cases however, such as when individuals interactions are localized, the expected waiting times are shown to be bounded. In this chapter, we explicitly characterize the convergence rates for stochastic evolution in networks and for positive noise levels. In particular, we characterize the expected waiting time as the measure of medium-run dynamics and the convergence time or selection time as the measure for long-run dynamics. We provide the characterization for convergence rates for both deterministic and random families of networks. We highlight how the topology of interactions acts to influence both the expected waiting time and convergence time.

Chapter 5 focuses on communication aspects of repeated interactions. It asks questions concerning the evolution of individual beliefs through repeated word-of-mouth communication with others in ones social network. Contrary to the predictions from the literature of rational learning that beliefs resulting from such a process of deliberation are homogeneous, disagreement among agents across social and economic settings is a common phenomenon. This then prompts a question of why such heterogeneities persist. Could it be a result of historical factors (prior beliefs), the learning process (the manner in which individuals incorporate new information into their beliefs), the topology of communication structure governing information exchange? Or could it be that the rate at which private information propagates through the population is too slow that we never get to observe an equilibrium state in which beliefs should be uniform? The learning mechanism depends on the assumptions 
made about individuals' cognitive abilities to perform the complex process of extracting private information from other agents' beliefs or actions. We refer to the case in which agents are capable of precisely extracting private information from others' beliefs as rational learning and that when they are not capable as boundedrational learning. More precisely, under rational learning agents are assumed to be able to extract private information from other agents' beliefs through Bayesian inference. Under bounded-rational learning on the other hand, for reasons that may involve lack of knowledge of informational and communication structures or simply the inability to perform complex inferences associated with Bayesian rational learning, we assume that agents incorporate other agents' beliefs by taking weighted averages. The speed at which learning occurs becomes important in "exit games" where individuals have to decide how long to wait before taking an action, or when the time available to gather information before making a decision is limited. This Chapter provides insights to these questions using a simple but robust theoretical model of social learning. 


\title{
2
}

\section{EPSILON STABILITY AND ROBUSTNESS IN STOCHASTIC EVOLUTIONARY GAMES ${ }^{1}$}

\begin{abstract}
Stochastic evolutionary models provide a useful framework for analyzing repeated interactions, experimentation and predictions concerning equilibrium selection. The commonly employed solution concept, stochastic stability, is still faced with numerous questions regarding its robustness and computational methods. This Chapter introduces a related but more general concept that we refer to as epsilon stability. An outcome is said to be epsilon stable if for a given level of experimentation it is the most stable in the long-run. Epsilon stability is an appropriate stability concept for stochastic evolution when the level of noise is positive as opposed to stochastic stability for limit noise. We then define robustness for stochastic stability as relating to the equivalence between epsilon and stochastically stable states. We provide conditions under which robustness exists. We also provide an efficient algorithm for computing both epsilon and stochastically stable sets. Application of the algorithm shows how risk-dominant strategies are more likely to be selected under multinomial logit than mistakes models. Dominant strategies however need not be selected in general.
\end{abstract}

\subsection{INTRODUCTION}

Multiplicity of equilibria is a well known phenomenon in economic models of strategic interactions. A typical example is economic environments with strategic complementarities, whereby the payoff a strategy or an action generates is a nondecreasing function of the number of players who adopt it. The question as to which among the possible equilibria gets selected or is much more likely in a long-run is of high interest in game theoretic modeling. In their pioneering work, Foster and Young [41], Kandori et al. [57] and Young [113] showed that a disequilibrium process in which agents learn their opponent's play and subsequently revise their strategies-generally known as stochastic evolutionary dynamics—can be employed as a mechanism for equilibrium selection. ${ }^{2}$ The basic idea of stochastic evolution is that

1 This Chapter has been published in UNU-MERIT working paper series: Opolot and Azomahou [91]

2 Since its development as an equilibrium selection mechanism, several applications and similar approaches to stochastic evolutionary dynamics (such as bargaining, signaling, contagion and social innovation) have 
individuals play their "optimal" strategies but the evaluation process is subjects to errors. The solution concept employed is stochastic stability according to Foster and Young [41]. An outcome is said to be stochastically stable if the probability with which it is played in the long-run is positive at the limit of noise.

Despite its effectiveness in predicting unique outcomes, several questions concerning robustness of stochastic stability remain unanswered. More specifically, for any given game the stochastically stable outcomes depend on the model of mistakes and the specifics concerning the nature of the dynamics. The related critical observation concerns implications of evolutionary dynamics analysis that focuses on limits of noise, especially in relations to the rates of convergence or time to selection. We argue in this Chapter that the most relevant question relates to robustness of stochastic stability to noise levels rather than the model of mistakes, which directly also relates to issues concerning the relevance of limit noise analysis. If stochastic stability is not robust to noise levels then caution needs to be taken when interpreting what stochastically stable sets imply. To fully capture this concept, we start by introducing the closely related concept to stochastic stability that we refer to as epsilon stability. Just like stochastically stable states or subsets are those which are the "most" stable in the long-run in the limit of noise, epsilon stable sets are those that are most stable in the long-run for positive noise levels. The first objective is then to derive methods and measures for computing epsilon stable sets. We do this in such a way that the measures for identifying stochastically stable sets are derived from those for identifying epsilon stable sets by simply taking limits of noise. We then say that stochastic stability is robust to noise for a given model of mistakes if there exists an equivalence between epsilon and stochastically stable sets. That is, the stochastically stable sets are those that are also epsilon stable.

In defining the model of stochastic evolution, the main assumptions we make are that players follow Darwinian dynamics and simultaneous revision protocol. The first assumption is customary in the literature and simply means that players do not possess infinite memory. The only relevant information in forming expectations at each period is the strategies of others in the previous period. There however exist approaches that consider infinite memory in the literature (generally called fictitious play) but with a focus on explaining how players learn to play Nash equilibrium rather than selection. The second assumption is mainly for computational reasons. Alós-Ferrer and Netzer [7] examine the robustness of stochastic stability to the revision protocol under logit dynamics, in which the probabilities of mistakes depend on

been explored. See for example Vega-Redondo [111], Nöldeke and Samuelson [88], Kandori and Rob [58], Alós-Ferrer and Weidenholzer [9], Huck et al. [53]. 
the payoff losses associated with the transition. They find the lack of robustness. That the states, hence strategies, that maximize the local potential, or equivalently that are stochastically stable under asynchronous revision protocol, are not necessarily those that are stochastically stable under independent simultaneous protocols. Blume [20] and Okada and Tercieux [89] identify this phenomenon as resulting from the peculiar characteristic of local potential functions and hence the logit dynamics. They show that if the payoffs are supermodular then stochastic stability is robust to the revision protocol. This phenomenon is also specific to cases in which probabilities of mistakes depend on payoff losses, such that in other cases like the mistakes model of Young [113], there is no particular reason as to why stochastic stability should not be robust to the revision protocol. The choice of the revision protocol however becomes very significant in determining the rates of convergence.

The approach we adopt in this Chapter circumvents some of the critical issues concerning stochastic stability and the relevance of considering limit analysis in stochastic evolutionary dynamics in the following ways. First, several authors have shown that the problems concerning selection time or expected waiting time to reach the stochastically stable sets from any other, is mitigated if players possess limited information. Limited information could be resulting from considering local interactions in which players observe strategies of only those in their social network (e.g. Ellison [37], Montanari and Saberi [77] and Young [115]. Recent work by Kreindler and Young [63] however shows that even under global interactions, selection could be fast provided noise levels are kept sufficiently positive. The concept of epsilon stability that we introduce is thus an appropriate solution concept for the setting with positive noise levels. The first contribution of this Chapter is thus providing the computational algorithm for epsilon stable sets.

Secondly, we analyze the question of robustness of stochastic stability to noise levels. We distinguish between two categories of models of mistakes; the mistakes model in which errors result from mutations, and the endogenous model of mistakes in which errors result from perturbations on payoffs, where we specifically focus on the multinomial logit model. For a given model of mistakes we then examine the robustness of stochastic stability to noise. The key assumption for the validity of stochastic stability is that the invariant distribution of the underlying stochastic process converges uniformly in the limit of noise. A violation of this assumption leads to arbitrary outcomes. In particular, under the mistakes model Bergin and Lipman [15] show that the long-run stable outcome of an evolutionary process strictly depends on the specifications made about the mutation rates. That it is possible to make any outcome stable in the long-run by suitably choosing the mutation rates structure. 
Here, by relating stochastic stability to epsilon stability, we provide conditions under which stochastic stability is robust to the mutation rates structure. In the case of endogenous mistakes model, and in particular the multinomial logit, the parameters that tune the noise level are state-independent and hence the effects identified by Bergin and Lipman [15] should not necessarily apply to this category of models. We indeed find robustness of stochastic stability to noise levels.

Finally, we provide efficient computational algorithms for both epsilons and stochastic stability that are independent of the model of mistakes. They make use of the quotients of invariant distributions, an idea originally introduced by Ellison [38] for identifying stochastically stable sets under the mistakes model. We extend this to the computation of epsilon stables sets and for endogenous mistakes models. The algorithmic steps we propose imply that the stochastic tree algorithm of Freidlin and Wentzell [42] that is normally employed in the literature but rather computationally complex, becomes necessary only after the quotients method fails to identify the unique epsilon and stochastically stable sets. But even so, the number of states that one has to construct their stochastic potentials can be drastically reduced by first employing the quotients method. With an exception of Alós-Ferrer and Netzer [7], the existing computation of stochastically stable sets under logit dynamics relies on the use of local potential maximization. But this method is specific to the case of asynchronous dynamics and as Alós-Ferrer and Netzer [7] pointed out, local potential maximizer is not necessarily stochastically stable under simultaneous revision protocol. It is thus relevant to develop simpler algorithms that are independent of the revision protocol. This Chapter equally makes a contribution in this direction and complements Alós-Ferrer and Netzer [7]. By applying our algorithm to both mistakes and multinomial logits models, it is shown states in which players play risk-dominant strategies are most likely to be epsilon and stochastically stable under the later than the former. But risk-dominant strategies need not be selected in both cases in general.

The remainder of the chapter is organized as follows. Section 2.2 provides a conceptual example demonstrating the non-robustness of stochastic stability and its relation to epsilon stability. In Section 2.3, we outline the general model for noisy stochastic evolutionary dynamics. Section 2.4 provides algorithmic characterization for epsilon and stochastic stability. Sections 2.5 and 2.6 are applications of the computational methods developed to the mistakes and multinomial logit models respectively. Section 2.7 is devoted to examples that illustrate the computational algorithm and the main results. Section 2.8 concludes the study. 
The following example illustrates the distinction we make between stochastic and epsilon stability using mutations model of mistakes. It demonstrates that unless the details of the model of mistakes are clearly defined, the result obtained by taking limits are not necessarily representative of small but finite noise behavior.

Consider a set of three players $\mathcal{N}=\{1,2,3\}$ who are uniformly and randomly matched to play the coordination game in Table 1 . Let the learning model be that defined in Young [113], in which each player follows the "Best Reply" dynamics with probability $1-\varepsilon$ and with probability $\varepsilon$ randomizes among the action set. Given the action set $X=\{a, b\}$, let $\mathbf{x}$ be the action profile, $U_{i}(x, \mathbf{x})$ be the payoff to $i \in \mathcal{N}$ when playing action $x$, and denote by $B R_{i}(x, \mathbf{x})$ for the probability that $i$ plays the Best Reply action. That is $B R_{i}(x, \mathbf{x})=1$ if $x \in \arg \max _{x_{i}} U_{i}\left(x_{i}, \mathbf{x}\right)$ and zero otherwise. Let $\mathcal{P}(x, \mathbf{x})$ be the mistakes distribution (or more specifically the probability mass function) identical to all players. Assume also that the payoff is identical for all players, such that the probability of playing action $x \in X$ given the mutation rate $\varepsilon$ and profile $\mathbf{x}$ is

$$
\mathbb{P}(x, \mathbf{x})=(1-\varepsilon) B R(x, \mathbf{x})+\varepsilon \mathcal{P}(x, \mathbf{x})
$$

From the payoff structure in Table $\mathbf{1}$, the states $\mathbf{a}$ and $\mathbf{b}$ in which all agents play $a$ and

Table 1: The action profile $(a, a)$ is risk-dominant

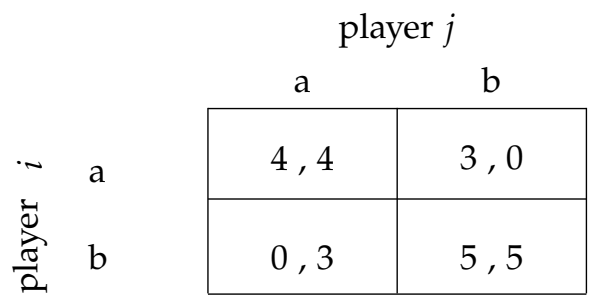

$b$ respectively are the equilibria (or limit states) of the best reply dynamics without mistakes. When players are matched uniformly and randomly, there are only four relevant states or action profiles; $\mathbf{a}, \mathbf{b}, b b a$ in which one agent plays $a$ and the other two play $b$, and $a a b$ in which one agent plays $b$ and the other two play $a$. Write $\mathbf{X}$ for the state space in the order $\mathbf{X}=(\mathbf{a}, a a b, b b a, \mathbf{b})$. Let $P_{\varepsilon}$ be the transition matrix of the Markov chain $\left(\mathbf{X}, P_{\varepsilon}\right)$ induced by the dynamics in (1). $P_{\varepsilon}$ is irreducible meaning that there exists a unique stationary distribution $\pi_{\varepsilon}$ whose structure is determined by the 
(normalized) left eigenvector corresponding to the leading eigenvalue of $P_{\mathcal{\varepsilon}}$. That is $\pi_{\varepsilon} P_{\varepsilon}=\pi_{\varepsilon}$.

The first step in the computation of the stochastically stable set normally is to determine the basins of attraction limit sets. From the payoff in Table 1 , it can easily be shown through best reply argument that the basin of attraction $\tilde{\mathbf{a}}$ of $\mathbf{a}$ is $\tilde{\mathbf{a}}=$ $\{\mathbf{a}, a a b, b b a\}$, and for $\mathbf{b}, \tilde{\mathbf{b}}=\{\mathbf{b}\}$. Let $e=\varepsilon \mathcal{P}(b, \mathbf{a}), f=\varepsilon \mathcal{P}(b, a a b)$ and $h=\varepsilon \mathcal{P}(a, \mathbf{b})$. Then the transition matrix $P_{\varepsilon}$ induced by the dynamics in (1) is given by,

$$
P_{\mathcal{\varepsilon}}=\left(\begin{array}{ccc:c}
(1-e)^{3} & 3(1-e)^{2} e & 3(1-e) e^{2} & e^{3} \\
(1-e)(1-f)^{2} & (1-f)(e+2 f-3 e f) & f(2 e+f-3 e f) & e f^{2} \\
(1-f)(2 f+h-3 f h) & (1-f)^{2}(1-h) & f^{2} h & f(f+2 h-3 f h) \\
\hdashline h^{3}-(1-h) h^{2} & 3(1-h)^{2} h & (1-h)^{3}-
\end{array}\right)
$$

The dashed lines partition $P_{\varepsilon}$ into block matrices describing transitions within basins of attraction (diagonal block matrices) and between basins of attraction (offdiagonal block matrices). The computation of stochastically stabel sets involves comparing the sizes of basins of attractions of limit sets or more generally minimum costs trees using the computational methods in Freidlin and Wentzell [42, Chapter 6]. Since the size of $\tilde{\mathbf{a}}$ is greater than that of $\tilde{\mathbf{b}}$, the stochastically stable state is $\mathbf{a}$.

Now, consider the case in which $\varepsilon=0.01$. Then there exists a mistakes distribution, for example $\mathcal{P}(b, \mathbf{a})=0.9, \mathcal{P}(b, a a b)=0.9$ and $\mathcal{P}(a, \mathbf{b})=10^{-5}$ for which $\pi(\mathbf{b})=$ $0.71>\pi(\mathbf{a})=0.28$. If $\varepsilon=10^{-4}$, then substituting $\mathcal{P}(b, \mathbf{a})=0.9, \mathcal{P}(b, a a b)=0.9$ and $\mathcal{P}(a, \mathbf{b})=10^{-9}$ into $P_{\mathcal{\varepsilon}}$ yields $\pi(\mathbf{b})=0.70>\pi(\mathbf{a})=0.29$.

This example shows that for every value of $\varepsilon \in(0,1)$ there exists a mistakes distribution for which an arbitrary limit set maximizes the stationary distribution or stable in the long-run. The state or subset of states that maximize the stationary distribution for a given model of mistakes and amount of noise is that we refer to as epsilon stable sets. The underlying reasoning behind stochastic stability is that for sufficiently small noise epsilon stable sets are equivalent to stochastically stable sets. But as this example shows and by other in the literature, it is not necessarily the case. For the special case of mutations model considered above, this reasoning holds whenever the probabilities $\mathcal{P}(x, \mathbf{x})=\frac{1}{m}$, where $m$ is the size of the action set. More generally however, it is safe to say that when the probabilities $\mathcal{P}(x, \mathbf{x})$ are bounded then there exists an $\varepsilon^{\prime}$ such that for any $\varepsilon<\varepsilon^{\prime}$, stochastic stability is equivalent to epsilon stability. We elaborate on this argument in section 2.5.

The general line of reasoning is as follows. First consider the case of the mutations model of mistakes as in ( 1 ) above. Let $\mathcal{P}(x, \mathbf{x})=\mathcal{P}$ be identical for all players and 
$\varepsilon=e^{-\beta}$ such $\varepsilon$ tending to zero implies $\beta$ tending to infinity. If $R(\mathbf{x}, \mathbf{y})$ is the number of mistakes required for the transition from (the limit state) $\mathbf{x}$ to $\mathbf{y}$ to occur, then

$$
P_{\mathcal{E}}(\mathbf{x}, \mathbf{y})=\left(\mathcal{P} e^{-\beta}\right)^{R(\mathbf{x}, \mathbf{y})}=\exp \{-(R(\mathbf{x}, \mathbf{y}) \beta-\ln \mathcal{P})\}
$$

such that

$$
\lim _{\beta \rightarrow \infty}-\frac{1}{\beta} \ln P_{\varepsilon}(\mathbf{x}, \mathbf{y})=R(\mathbf{x}, \mathbf{y})
$$

Implying that $R(\mathbf{x}, \mathbf{y})$ is the decay rate of transition probabilities between limit sets. The existing computational methods for stochastic stability rely on the assumption that these decay rates are uniform and depend on only values of $R(\mathbf{x}, \mathbf{y})$, which are also sizes of basins of attraction. The cost of getting from one limit state or subset of states to another is then computed by adding up the decay rates associated with such a path. Note however that in (2) we assumed that $R(\mathbf{x}, \mathbf{y})$ are not only identical but also finite, otherwise it does not hold. When computing epsilon stable states on the other hand we consider the entire quantity $R(\mathbf{x}, \mathbf{y}) \beta-\ln \mathcal{P}$ for a given level of noise $\beta$.

Now consider the case of logit choice probabilities commonly employed in the literature. For the game in Table $\mathbf{1}$ where $\mathbf{a}$ and $\mathbf{b}$ are the limit states, we have

$$
\begin{aligned}
\mathbb{P}(a, \mathbf{b})=\frac{\exp (\beta U(a, \mathbf{b}))}{\exp (\beta U(a, \mathbf{b}))+\exp (\beta U(b, \mathbf{b}))} & =\frac{1}{1+\exp (\beta[U(b, \mathbf{b})-U(a, \mathbf{b})])} \\
& \approx \exp \left(-\beta \Delta U_{b a}(\mathbf{b})\right)
\end{aligned}
$$

where $\Delta U_{b a}(\mathbf{b})=U(b, \mathbf{b})-U(a, \mathbf{b})$. The approximation is for $\beta$ sufficiently large. Implying that

$$
P_{\varepsilon}(\mathbf{b}, \mathbf{a})=\left(\exp \left(-\beta \Delta U_{b a}(\mathbf{b})\right)\right)^{R(\mathbf{b}, \mathbf{a})}=\exp \left\{-R(\mathbf{b}, \mathbf{a}) \Delta U_{b a}(\mathbf{b}) \beta\right\}
$$

such that

$$
\lim _{\beta \rightarrow \infty}-\frac{1}{\beta} \ln P_{\mathcal{\varepsilon}}(\mathbf{b}, \mathbf{a})=R(\mathbf{b}, \mathbf{a}) \Delta U_{b a}(\mathbf{b})
$$

Similarly,

$$
\lim _{\beta \rightarrow \infty}-\frac{1}{\beta} \ln P_{\mathcal{E}}(\mathbf{a}, \mathbf{b})=R(\mathbf{a}, \mathbf{b}) \Delta U_{a b}(\mathbf{a})
$$

The decay rates of transition probabilities for logit dynamics thus depend on both the sizes of basins of attraction and payoff differences. When computing the stochastically stable states, the cost of getting from one limit state to another thus is the sum of the quantities $R(\mathbf{a}, \mathbf{b}) \Delta U_{a b}(\mathbf{a})$ of the associated path. This implies that for given sizes of basins of attraction, states that incur higher payoff losses to exit are more stable. Since risk-dominant strategies exhibit larger payoff losses, it is precisely why 
risk-dominant strategies are stochastically stable in $2 \times 2$ matrix games under logit dynamics (e.g Maruta [71], Blume [20], Okada and Tercieux [89]). This is not necessarily true for (2) above. When computing epsilon stable sets in this specific case of logit dynamics, we would have to consider the quantities $R(\mathbf{x}, \mathbf{y}) \Delta U_{x y}(\mathbf{x}) \beta$.

The basis of the analysis in this Chapter is that given any model of mistakes, for any pair of limit sets say $\mathbf{x}$ and $\mathbf{y}$, the transition probabilities can be expressed as

$$
P_{\mathcal{E}}(\mathbf{x}, \mathbf{y})=K \exp \left\{R(\mathbf{x}, \mathbf{y}) \ln \mathbb{P}_{A}(\mathbf{x}, \mathbf{y})+\mathcal{O}(1)\right\}
$$

where $K$ is some constant and $\mathbb{P}_{A}(\mathbf{x}, \mathbf{y})=\frac{1}{N} \sum_{i=1}^{N} \mathbb{P}\left(y^{i}, \mathbf{x}\right)$

\subsection{THE MODEL}

We study the class of 2 -persons $m \times m$ matrix games. The set of players is denoted by $\mathcal{N}=\{1, \cdots, i, \cdots, N\}$ the action set by $X_{i}=\{a, b, \cdots\}$ for each player. Denote by $t=1,2, \cdots$ for the successive periods of play in which players simultaneously play the game. We also assume that players are randomly matched such that at each period each observes the previous period's strategies of the entire population. The resulting population state or profile at period $t$ will be denoted by $\mathbf{x}_{t}$ and the set of all possible such realizations by $\mathbf{X}=\prod_{i=1}^{n} X_{i}$.

For each game, let $U \in \mathbb{R}^{m \times m}$ be the associated payoff matrix such that $U_{i}(a, b)$ is the base payoff to the player playing $a$ when his opponent plays $b$. A typical example is the pure coordination game in Table 12. Given the information set of agent $i$, that is the observed distribution of strategies $\mathbf{x}$, the expected payoff to $i$ from playing action $a$ when his opponents profile is $\mathbf{x}_{-i}$ is then

$$
U_{i}\left(a, \mathbf{x}_{-i}\right)=\sum_{j \in \mathcal{N}} J_{i j} U\left(a, x^{j}\right)
$$

where $x^{j}$ is the $j^{\text {th }}$ coordinate of $\mathbf{x}$. The parameter $J_{i j}$ takes on values in the closed interval $[0,1]$. For example if agents consider average payoff over their opponents' strategies, then $J_{i j}=\frac{1}{N}$ for all $j \in \mathcal{N}$.

\subsubsection{Dynamics and learning scheme}

There are two main components that completely describe the learning process; the revision protocol and choice probabilities. We consider the simultaneous revision protocol for reasons mentioned in the introduction. When a player receives a revision opportunity, they evaluate the current expected payoff to each of the available pure 


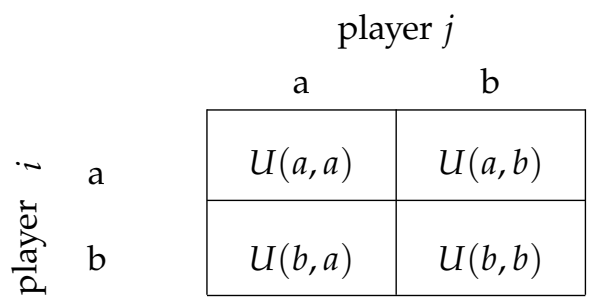

Table 2: Payoff structure for the symmetric coordination game between $i$ and $j$. The indicated payoffs are for the row-player

strategies. Each player's objective is to select strategies that are evaluated as best, this assessment is however subject to random shocks. The random shocks can either be in form of perturbations on the payoffs or strategies. Under the former, $i$ 's objective is to choose a strategy $a$ that maximizes the perturbed payoff $U_{i}\left(a, \mathbf{x}_{-i}\right)+\varepsilon_{a}$, that is

$$
a \in \underset{b \in X}{\arg \max } U_{i}\left(b, \mathbf{x}_{-i}\right)+\varepsilon_{b}
$$

where $\varepsilon_{b}$ 's are the random components of the expected payoff. The components of $\varepsilon=\left(\varepsilon_{a}, \varepsilon_{b}, \cdots\right)$ are assumed to be independently distributed. The conditional (on the realization of $\varepsilon_{a}$ ) perturbed best response function for $i$ is given by the distribution

$$
\mathbb{P}_{i}\left(a, \mathbf{x} ; \varepsilon_{a}\right)=\mathbb{P}\left(\bigcap_{b \neq a}\left\{U_{i}\left(a, \mathbf{x}_{-i}\right)+\varepsilon_{a} \geq U_{i}\left(b, \mathbf{x}_{-i}\right)+\varepsilon_{b}\right\}\right)
$$

In the case where perturbations are on agents' strategies/actions, the perturbed best response function is given by,

$$
\mathbb{P}_{i}(a, \mathbf{x})=\left(1-\varepsilon_{i}(\mathbf{x})\right) B R_{i}(a, \mathbf{x})+\varepsilon_{i}(\mathbf{x}) \mathcal{P}_{i}(a, \mathbf{x})
$$

where $B R_{i}(a, \mathbf{x})$ is the probability that $i$ plays action $a$ under best-reply dynamics given that the population state $\mathbf{x}$. That is

$$
B R_{i}(a, \mathbf{x})= \begin{cases}1 & \text { if } a \in \arg \max _{b \in X} U_{i}\left(b, \mathbf{x}_{-i}\right) \\ 0 & \text { otherwise. }\end{cases}
$$

For each $i \in \mathcal{N}, \varepsilon_{i}(\mathbf{x})$ is the state-dependent mutation rate, the probability that $i$ randomizes among the elements of $X$ with the conditional distribution defined by $\mathcal{P}_{i}(a, \mathbf{x})$. The product $\mathscr{P}_{i}(a, \mathbf{x})=\varepsilon_{i}(\mathbf{x}) \mathcal{P}_{i}(a, \mathbf{x})$ is the probability of playing action $a$ by mistake. We denote the vector of mutation rates by $\varepsilon=\left(\varepsilon_{1}(\mathbf{x}), \cdots, \varepsilon_{n}(\mathbf{x})\right)$. Note that for each $i \in \mathcal{N}$ and $a \in X, \sum_{a \in X} \mathcal{P}_{i}(a, \mathbf{x})=1$. 
The dynamics (8) in which payoffs are perturbed has been studied extensively in evolutionary game theory. See for example Hofbauer and Sandholm [50] and Hofbauer and Sandholm [51] who prove the general equilibrium existence for such dynamics. The special cases are the logit, and multinomial probit in Blume [20] and Myatt and Wallace [82] respectively. The special case of dynamics (9) are Kandori et al. [57] and Young [113] where mutation rates are state and player independent.

Each of the above dynamics defines a Markov chain on a finite state space $\mathbf{X}$ whose transition matrix $P_{\varepsilon}$ is defined by transition probabilities $P_{\varepsilon}(\mathbf{x}, \mathbf{y})$,

$$
P_{\mathcal{\varepsilon}}(\mathbf{x}, \mathbf{y})=\prod_{i=1}^{n} \mathbb{P}_{i}\left(y^{i}, \mathbf{x}\right)
$$

\subsubsection{Stochastic and epsilon stability}

To distinguish between the Markov chain without noise, which derives from the dynamics in (10), and that with noise we use the notations $(\mathbf{X}, P)$ and $\left(\mathbf{X}, P_{\varepsilon}\right)$ respectively. Chain $(\mathbf{X}, P)$ consists of limit or closed communication classes that we denote by $\Omega$, and the set of all such subsets by $\Omega .^{3}$ In the presence of noise, the closed communication classes of $(\mathbf{X}, P)$ become metastable. That is the chain $\left(\mathbf{X}, P_{\mathcal{E}}\right)$ spends an extended amount of time in each closed communication class but will eventually transition to another. To avoid notational and conceptual clutter, we equally use closed communication classes to refer to metastable sets since at sufficiently low levels of noise their compositions are equivalent.

The perturbed Markov chain $\left(\mathbf{X}, P_{\mathcal{E}}\right)$ has a unique invariant (stationary) distribution $\pi_{\varepsilon}=\lim _{t \rightarrow \infty} \mathbf{q}_{0} P_{\varepsilon}^{t}$, where $\mathbf{q}_{t}$ is the vector of probability mass functions at period $t$. It describes the amount of time the process spends in each state in the long-run. The underlying notion of stochastic stability is that the states or subsets of states that are not stochastically stable will be observed less frequently compared to those that are. Formally (definition from Foster and Young [41]),

Definition 1: Given the chain $\left(\mathbf{X}, P_{\mathcal{E}}\right)$, the closed communication class $\Omega$ is stochastically stable if $\lim _{\mathcal{\varepsilon} \rightarrow 0} \pi_{\mathcal{\varepsilon}}(\Omega)>0$.

Clearly for any given game, the stochastically stable states (hence strategies) depend not only on the model of mistakes. This distinction would not be relevant if stochastically stability is robust to both the model of mistakes and the noise levels.

3 A set $\Omega \subset \mathbf{X}$ is a limit set of $(\mathbf{X}, P)$ if $\forall \mathbf{y} \in \Omega, \mathbb{P}\left(\mathbf{x}_{t+1} \in \Omega \mid \mathbf{x}_{t}=\mathbf{y}\right)=1$, and that $\forall \mathbf{y}, \mathbf{z} \in \Omega$, there exists a $\mathscr{T}>0$ such that $\mathbb{P}\left(\mathbf{x}_{t+\mathscr{T}}=\mathbf{z} \mid \mathbf{x}_{t}=\mathbf{y}\right)>0$. 
This is however not the case as argued in Sections 2.1 and 2.2 above. For this reason, we introduce a related concept of epsilon stability. Formally,

Definition 2: Given $\left(\mathbf{X}, P_{\varepsilon}\right)$, a subset $\Omega^{*}$ is said to be epsilon stable if

$$
\Omega^{*}=\arg \max _{\Omega \in \Omega} \pi_{\varepsilon}(\Omega)
$$

An epsilon stable set can thus be equivalently defined as that for which $\pi_{\varepsilon}\left(\Omega^{*}\right)>$ $\pi_{\varepsilon}(\Omega)$ for all $\Omega \neq \Omega^{*}$. A stochastically stable set is epsilon stable only under some restrictions on the model of mistakes. If for a given model of mistakes the stochastically stable sets are also those that are epsilon stable, then we say there exists an equivalence between stochastic and epsilon stability. If this equivalence is true for all level of noise then we say that stochastic stability is robust, otherwise robustness exists for only some levels of noise.

We use the following additional notations for the remainder of the Chapter. The boundary of a closed communication class $\Omega$ will be denoted by $\partial \Omega$. That is, all states in the basin of attraction of $\Omega$ with $\Omega$ excluded. ${ }^{4}$ The basin of attraction of $\Omega$ is then $\tilde{\Omega}=\partial \Omega \cup \Omega$. We write $\left(\tilde{\Omega}, \tilde{P}_{N, \varepsilon}\right)$ for the chain with transitions between elements $\tilde{\Omega}$.

\subsection{STOCHASTIC AND EPSILON STABLE SETS}

The question that follows the above definitions is how to compute states or subsets of states that are stochastically and epsilon stable, and if there exists a relationship between the two. In other words, when should we expect epsilon stable sets to also be stochastically stable. As discussed above, this will of course depend on the specific model of mistakes. But for a given model of mistakes, we seek for conditions under which an equivalence exists between stochastic and epsilon stability.

There are two methods for computing stochastically stable sets which directly extend to the computation of epsilon stable sets. The first considers quotients of stationary distributions values and the other involves a complete characterization of the stationary distribution using the combinatorial methods of Freidlin and Wentzell [42]. The quotients methods is computationally less complex but coarser than the tree algorithms associated with the combinatorial methods. For most stochastic evolutionary setups however, the quotient method is sufficient. Hence the most efficient computational algorithm would be that which combines both methods in the ascending order of computational complexity. We begin by elaborating on the quotients method.

4 The basin of attraction of a closed communication class $\Omega$ is defined as $\tilde{\Omega}=\left\{\mathbf{x} \in \mathbf{X} \mid \mathbb{P}\left(\exists T\right.\right.$ s.t $\mathbf{x}_{t} \in \Omega \forall t>$ $\left.\left.T \mid \mathbf{x}_{0}=\mathbf{x}\right)=1\right\}$. That is, the set of states from which the chain without noise converges to $\Omega$. 
LeMMA 1: Let $\Omega$ and $\Omega^{\prime}$ be any two closed communication classes such that $P_{\varepsilon}\left(\Omega^{\prime}, \Omega\right)$ is the probability of the transition from some state in $\Omega^{\prime}$ to another in $\Omega$. Let also $\Omega^{c}$ be the complement of $\Omega$. Then

$$
\frac{\pi_{\varepsilon}\left(\tilde{\Omega}^{\prime}\right)}{\pi_{\mathcal{E}}(\tilde{\Omega})} \leq \frac{P_{\mathcal{\varepsilon}}\left(\Omega, \Omega^{c}\right)}{P_{\mathcal{E}}\left(\Omega^{\prime}, \Omega\right)} \approx \frac{\max _{\Omega^{\prime \prime}} P_{\mathcal{\varepsilon}}\left(\Omega, \partial \Omega^{\prime \prime}\right)}{P_{\mathcal{E}}\left(\Omega^{\prime}, \partial \Omega\right)}
$$

Proof. See Appendix 3.8.1

The probabilities $P_{\mathcal{\varepsilon}}\left(\Omega, \Omega^{\prime}\right)$ are in principle single step probabilities involving states belonging to two different closed communication classes. The quotients method in (3.3.1) is coarse in the sense that it may not necessarily identify the unique long-run stable states. It is however possible to make it finer by extending it to paths rather than just binary relations. This can be achieved by use of the chain-rule argument. That is, if there exists an intermediate closed communication class $\Omega^{\prime \prime}$ then,

$$
\frac{\pi_{\varepsilon}\left(\tilde{\Omega}^{\prime}\right)}{\pi_{\varepsilon}(\tilde{\Omega})}=\frac{\pi_{\varepsilon}\left(\tilde{\Omega}^{\prime}\right)}{\pi_{\varepsilon}\left(\tilde{\Omega}^{\prime \prime}\right)} \frac{\pi_{\varepsilon}\left(\tilde{\Omega}^{\prime \prime}\right)}{\pi_{\varepsilon}(\tilde{\Omega})} \leq \frac{P_{\mathcal{\varepsilon}}\left(\Omega^{\prime \prime}, \Omega^{\prime \prime c}\right)}{P_{\mathcal{E}}\left(\Omega^{\prime}, \Omega^{\prime \prime}\right)} \frac{P_{\mathcal{\varepsilon}}\left(\Omega, \Omega^{c}\right)}{P_{\mathcal{\varepsilon}}\left(\Omega^{\prime \prime}, \Omega\right)}
$$

In which case the chain starts from $\Omega^{\prime}$ then to $\Omega^{\prime \prime}$ and finally to $\Omega$.

The second technique for computing long-run stable sets follows from the combinatorial methods of Freidlin and Wentzell [42]. Given the Markov chain $\left(\mathbf{X}, P_{\varepsilon}\right)$, let $W \subset \mathbf{X}$ be a subset of $\mathbf{X}$ and $W^{c}=\mathbf{X} \backslash W$ its complement. For any oriented graph $g \subset \mathbf{X} \times \mathbf{X}$ and any $\mathbf{x} \in \mathbf{X}$, write $g(\mathbf{x})=\{\mathbf{y}:(\mathbf{x}, \mathbf{y}) \in g\}$ denote the immediate successors of $\mathbf{x}$. More generally $g^{n}(\mathbf{x})=\bigcup_{\mathbf{y} \in g^{n-1}(\mathbf{y})} g(\mathbf{x})$.

Definition 3: Let $G(W)$ be the set of oriented graphs $g \subset \mathbf{X} \times \mathbf{X}$ satisfying

1. for any $\mathbf{x} \in \mathbf{X}, \# g(\mathbf{x})=\mathbf{1}_{W^{c}}$ (that is no arrows start from $W$ and exactly on arrow starts from each state outside of $W$ ),

2. for any $\mathbf{x} \in \mathbf{X}, \mathbf{x} \notin \mathcal{O}_{g}(\mathbf{x})$, where $\mathcal{O}_{g}(\mathbf{x})=\bigcup_{n=1}^{+\infty} g^{n}(\mathbf{x})$ is the the set of points that can be reached from $\mathbf{x}$; the orbit of $\mathbf{x}$ under $g$ (that is $g$ has no loops).

From the definition of $G(W)$ graphs above, $G(\{\mathbf{x}\})$ is the set of all spanning trees in which there exists a unique directed path from every $\mathbf{y} \neq \mathbf{x}$ to $\mathbf{x}$, also known as $\mathbf{x}$-trees. The following Lemma is proved in Freidlin and Wentzell [42] (see also Catoni [26]).

LemMa 2: The unique invariant distribution of $P_{\varepsilon}$ is,

$$
\pi_{\mathcal{\varepsilon}}(\mathbf{x})=\left(\sum_{g \in G(\{\mathbf{x}\})} P_{\mathcal{\varepsilon}}(g)\right)\left(\sum_{\mathbf{y} \in \mathbf{X}} \sum_{g \in G(\{\mathbf{y}\})} P_{\mathcal{\varepsilon}}(g)\right)^{-1}
$$

where $P_{\mathcal{\varepsilon}}(g)=\prod_{(\mathbf{x}, \mathbf{y}) \in g} P_{\mathcal{\varepsilon}}(\mathbf{x}, \mathbf{y})$. 
Similarly, if we consider the graph defined on the space $\Omega$, then

$$
\pi_{\mathcal{\varepsilon}}(\Omega)=\left(\sum_{g \in G(\{\Omega\})} P_{\mathcal{E}}(g)\right)\left(\sum_{\Omega^{\prime} \in \tilde{\Omega}} \sum_{g \in G\left(\left\{\Omega^{\prime}\right\}\right)} P_{\mathcal{\varepsilon}}(g)\right)^{-1}
$$

where $P_{\mathcal{\varepsilon}}(g)=\prod\left(\Omega, \Omega^{\prime}\right) \in g\left(P_{\mathcal{\varepsilon}}\left(\Omega, \Omega^{\prime}\right)\right.$.

In both cases, the quotients and tree algorithmic methods, the most relevant quantities are the probabilities $P_{\mathcal{E}}\left(\Omega, \Omega^{\prime}\right)$ which is approximately equivalent to $P_{\mathcal{E}}\left(\Omega, \partial \Omega^{\prime}\right)$ (see Lemma 5). The following proposition provides the bounds for the $P_{\mathcal{\varepsilon}}\left(\Omega, \partial \Omega^{\prime}\right)$ for any pair of closed communication classes. For each pair of closed communication classes, $R\left(\Omega, \partial \Omega^{\prime}\right)$ is the number of players required to play action $x_{\Omega^{\prime}}$ by mistake for the transition $\Omega \rightarrow \partial \Omega^{\prime}$ to occur, such that $\mathbb{P}_{i}\left(x_{\Omega^{\prime}}, \Omega\right)$ is the probability that player $i$ plays $x_{\Omega^{\prime}}$ given that the chain is in some state of $\Omega$. We also write $r\left(\Omega, \partial \Omega^{\prime}\right)=\frac{1}{N} R\left(\Omega, \partial \Omega^{\prime}\right)$ and $\mathbb{P}_{A}\left(x_{\Omega^{\prime}}, \Omega\right)=\frac{1}{N} \sum_{i=1}^{N} \mathbb{P}_{i}\left(x_{\Omega^{\prime}}, \Omega\right)$.

Proposition 1: The probability $P_{N, \varepsilon}\left(\Omega, \partial \Omega^{\prime}\right)$ of the transition from a state in $\Omega$ to another in $\partial \Omega^{\prime}$ in a single time step is bounded by

$$
\begin{aligned}
K_{P} \exp \left\{-N\left(c\left(\Omega, \partial \Omega^{\prime}\right)+F\left(r\left(\Omega, \partial \Omega^{\prime}\right)\right)\right)\right\} & \leq P_{N, \varepsilon}\left(\Omega, \partial \Omega^{\prime}\right) \\
& \leq \exp \left\{-N\left(c\left(\Omega, \partial \Omega^{\prime}\right)+F\left(r\left(\Omega, \partial \Omega^{\prime}\right)\right)\right)\right\}
\end{aligned}
$$

where $c\left(\Omega, \partial \Omega^{\prime}\right)=-r\left(\Omega, \partial \Omega^{\prime}\right) \ln \mathbb{P}_{A}\left(x_{\Omega^{\prime}}, \Omega\right), F(r)=r \ln \left(\frac{r}{1-r}\right)+\ln (1-r)$ and $K_{P}$ is some constant.

Proof. The details of the proof are relegated to the Appendix 2.9.2 and its sketch is as follows. Given $x_{\Omega^{\prime}}$, the action that must be played by mistake for the transition $\Omega \rightarrow \partial \Omega^{\prime}$ to occur, Let $N_{t}\left(x_{\Omega^{\prime}}\right)$ be the number of players playing $x_{\Omega^{\prime}}$ at period $t$. Then $P_{\mathcal{E}}\left(\Omega, \partial \Omega^{\prime}\right)$ can be equivalently expressed as

$$
P_{\mathcal{\varepsilon}}\left(\Omega, \partial \Omega^{\prime}\right)=\mathbb{P}\left(N_{t+\tau}\left(x_{\Omega^{\prime}}\right) \geq R\left(\Omega, \partial \Omega^{\prime}\right) \mid \mathbf{x}_{t} \in \Omega\right)
$$

That is, the probability that at least $R\left(\Omega, \partial \Omega^{\prime}\right)$ players simultaneously play $x_{\Omega^{\prime}}$ after $\tau$ periods given that the process starts from some state in $\Omega$. For the case of simultaneous revision protocol considered here, $\tau=1$. To derive bounds for the right hand side of (3.3.3), we first transform the problem into that of Bernoulli random variables. That is, if we let $I_{i}$ be a binary random variable taking on values 1 with probability $\mathbb{P}_{i}\left(x_{\Omega^{\prime}}, \Omega\right)$ and zero otherwise, then

$$
\mathbb{P}\left(N_{t+1}\left(x_{\Omega^{\prime}}\right) \geq R\left(\Omega, \partial \Omega^{\prime}\right) \mid \mathbf{x}_{t} \in \Omega\right) \equiv \mathbb{P}\left(\sum_{i=1}^{N} I_{i} \geq R\left(\Omega, \partial \Omega^{\prime}\right)\right)
$$

The structure of bounds for $\mathbb{P}\left(\sum_{i=1}^{N} I_{i} \geq R\left(\Omega, \partial \Omega^{\prime}\right)\right)$ can then be established using techniques for deriving concentration bounds. If the individual probabilities of 
making a mistake were identical then the concentrations bounds would directly follow from the large deviation results for Bernoulli random variables. For the case of heterogeneity of mistakes probabilities, a constructive proof's approach is the appropriate method. The details of the steps that follow are in Appendix 2.9.2.

Each $c\left(\Omega, \partial \Omega^{\prime}\right)$ is the cost of the transition $\Omega \rightarrow \partial \Omega^{\prime}$. It is a function of the number of mistakes required for such a transition to occur and the actual probability of mistakes. The following shorthand and definitions are used for the Sections that follow. First, we write $Q\left(\Omega, \Omega^{\prime}\right)=c\left(\Omega, \partial \Omega^{\prime}\right)+F\left(r\left(\Omega, \partial \Omega^{\prime}\right)\right), Q_{\min }(\Omega)=\min _{\Omega^{\prime} \neq \Omega} Q\left(\Omega, \Omega^{\prime}\right)$ and $Q_{\max }(\Omega)=\max _{\Omega^{\prime} \neq \Omega} Q\left(\Omega^{\prime}, \Omega\right)$. Such that $Q(\Omega)=Q_{\max }(\Omega)-Q_{\min }(\Omega)$. Secondly, we define the paths in relation to the expression in (13). Let $\mathscr{H}_{\Omega_{1} \Omega_{J}}$ be the set of all directed paths starting from $\Omega_{1}$ and terminating at $\Omega_{J}$, and let $H_{\Omega_{1} \Omega_{J}}=$ $\left(\Omega_{1}, \cdots, \Omega_{j}, \cdots, \Omega_{J}\right)$ be the typical path in $\mathscr{H}_{\Omega_{1} \Omega_{J}}$. Then the path potential $\xi\left(H_{\Omega_{1} \Omega_{J}}\right)$ of $H_{\Omega_{1} \Omega_{J}}$ is defined as

$$
\xi\left(H_{\Omega_{1} \Omega_{J}}\right)=\sum_{j=1}^{J-1}\left(Q\left(\Omega_{j}, \Omega_{j+1}\right)-Q_{\min }\left(\Omega_{j+1}\right)\right)
$$

That is, the total cost of the path minus the total of minimum deviations from the path. The path potential is thus a measure of how accessible or reachable a given closed communication class is from another through that particular path. The logic behind the definition is that the accessibility of $\Omega_{J}$ from $\Omega_{1}$ through $H_{\Omega_{1} \Omega_{J}}$ depends on the total cost associated with $H_{\Omega_{1} \Omega_{J}}$ and the likelihood of deviating from $H_{\Omega_{1} \Omega_{J}}$. The higher the cost the more difficult it is to reach $\Omega_{J}$ through $H_{\Omega_{1} \Omega_{J}}$ and the lower the resistance of the basins of attraction that the path traverses to reach $\Omega_{J}$ the more likely that the process will follow such a path.

We then define the maximum path potential of any given closed communication class $\Omega_{J}$ as follows

$$
\xi\left(\Omega_{J}\right)=\max _{\Omega_{j} \neq \Omega_{J}} \min _{\Omega_{\Omega_{j} \Omega_{J}} \in \mathscr{H}_{\Omega_{j} \Omega_{J}}} \xi\left(H_{\Omega_{j} \Omega_{J}}\right)
$$

Thirdly, we define the measure related to the tree algorithm. Given the definition of $G(W)$ graphs above, the stochastic potential of $\Omega$ is then defined as

$$
\mathscr{S}(\Omega)=\min _{g \in G(\{\Omega\})} \sum_{\left(\Omega_{j}, \Omega_{\kappa}\right) \in g} Q\left(\Omega_{j}, \Omega_{\kappa}\right)
$$

THeOREM 1: For the process $P_{\varepsilon}$, if $\bar{\Omega}$ is the subset containing the epsilon stable state then,

(i) $Q(\bar{\Omega})<0$,

(ii) $\xi(\bar{\Omega})<0$ and 
(iii) $\mathscr{S}(\bar{\Omega})=\min _{\Omega \in \Omega} \mathscr{S}(\Omega)$.

Proof. See Appendix 2.9.3

Theorem 1 provides algorithmic steps for computing the epsilon stable sets. It involves three measures derived from quotients rule and the spanning tree algorithms. An efficient algorithm is then that combines the three measures in the ascending order of computational complexity. More specifically, it consists of the following steps.

The first step is to identify the closed communication classes for the given underlying game together with the associated basins of attraction. This can usually be done heuristically from the underlying payoff structure. In the process of identifying the closed communication classes and basins of attraction, one also identifies the respective action that players should play for the transition to occur, and hence the associated average probabilities. The second step involves computing the quantities $Q(\bar{\Omega})$ for each closed communication class and identifying those for which $Q(\bar{\Omega})<0$. If this procedure identifies one closed communication class, then it is the unique epsilon stable set. If the procedure leads to a null set, then compute the maximum path potentials for each closed communication class and identify those for which the potential is negative. A set that is a union of all closed communication classes whose maximum path potential are negative, contains the epsilon stable set. If this procedure identifies one closed communication class, then it is the unique epsilon stable set. If either of the procedures selects more than one closed communication class then proceed to the third step, which employs the spanning tree algorithm to identify the epsilon stable set. More specifically, compute the stochastic potentials of all sets that survived the second step and select those with the minimum stochastic potential.

The spanning tree algorithm is therefore only necessary if the first two steps fail to select a unique epsilon stable set. Moreover, the first two steps reduce the number of communication classes for which one has to construct their spanning trees. This tremendously reduces the computational burden of constructing the spanning trees for each closed communication class and the associated stochastic potentials.

To compute stochastically stable sets, we take the limits of transitions between closed communication classes. From Proposition 4

$$
\lim _{\varepsilon \rightarrow 0}-\frac{\varepsilon}{N} \ln P_{\varepsilon}\left(\Omega, \partial \Omega^{\prime}\right)=\lim _{\varepsilon \rightarrow 0} \varepsilon Q\left(\Omega, \Omega^{\prime}\right)=V\left(\Omega, \Omega^{\prime}\right)
$$

Since $-0.7 \leq F(r) \leq 0$ and independent of the noise parameters, then $V\left(\Omega, \Omega^{\prime}\right)=$ $\lim _{\varepsilon \rightarrow 0} \varepsilon c\left(\Omega, \partial \Omega^{\prime}\right)$. Following the same definitions and measures above, we write 
$V_{\min }(\Omega)=\min _{\Omega^{\prime} \neq \Omega} V\left(\Omega, \Omega^{\prime}\right)$ and $V_{\max }(\Omega)=\max _{\Omega^{\prime} \neq \Omega} V\left(\Omega^{\prime}, \Omega\right)$. Such that $V(\Omega)=$ $V_{\max }(\Omega)-V_{\min }(\Omega)$. Similarly,

$$
\begin{gathered}
\xi_{\mathcal{S}}\left(H_{\Omega_{1} \Omega_{J}}\right)=\sum_{j=1}^{J-1}\left(V\left(\Omega_{j}, \Omega_{j+1}\right)-V_{\min }\left(\Omega_{j+1}\right)\right) \\
\xi_{S}\left(\Omega_{J}\right)=\max _{\Omega_{j} \neq \Omega_{J}} \min _{H_{\Omega_{j} \Omega_{J}} \in \mathscr{H}_{\Omega_{j} \Omega_{J}}} \xi_{\mathcal{S}}\left(H_{\Omega_{j} \Omega_{J}}\right) \\
\mathscr{S}_{S}(\Omega)=\min _{g \in G(\{\Omega\})} \sum_{\left(\Omega_{j}, \Omega_{\kappa}\right) \in g} V\left(\Omega_{j}, \Omega_{\kappa}\right)
\end{gathered}
$$

Corollary 1: For the process $P_{\varepsilon}$, if $\Omega^{*}$ is the subset containing the stochastically stable state then,

(i) $V\left(\Omega^{*}\right)<0$,

(ii) $\xi_{s}\left(\Omega^{*}\right)<0$ and

(iii) $\mathscr{S}_{S}\left(\Omega^{*}\right)=\min _{\Omega \in \Omega} \mathscr{S}_{S}(\Omega)$.

The efficient algorithm for computing stochastically stable sets thus involves similar steps as for epsilon stable sets with the only difference being the quantities $Q\left(\Omega, \Omega^{\prime}\right)$ and $V\left(\Omega, \Omega^{\prime}\right)$. The following sections provide applications of Theorem 1 and Corollary 1 to specific choice probabilities. More specifically, the mistakes and the multinomial logit models.

\subsection{THE MISTAKES MODEL}

The key property of the mistakes model in (4.2.2) is that the probability of making a mistake to play an action that may or may not be optimal is a function of the mutation rate $\varepsilon_{i}$ and probability distribution over the action set $\mathcal{P}_{i}(x, \mathbf{x})$. As demonstrated in the conceptual example above, both epsilon and stochastically stable sets depend on the structure of $\mathcal{P}_{i}(x, \mathbf{x})$. The following proposition provides conditions under which the epsilon stable sets are also those that are stochastically stable.

Proposition 2: Given the set of mutation rates $\varepsilon$ that are state independent and the size of action set to be $m$, let $0<\mathcal{P}_{i}(x, \mathbf{x})<1 \forall i \in \mathcal{N}, \forall x \in X$ and $\forall \mathbf{x} \in \mathbf{X}$.

(i) If $\mathcal{P}_{i}(x, \mathbf{x})=\frac{1}{m} \forall i, \forall x \in X$ and $\forall \mathbf{x} \in \mathbf{X}$, then for all values of $\varepsilon_{i}$ for all $i$, the epsilon stable sets are also those that are stochastically stable.

(ii) If $\mathcal{P}_{i}(x, \mathbf{x}) \neq \frac{1}{m}$, then there exists an $\varepsilon^{\prime}>0$ such that for all $\varepsilon_{i}<\varepsilon^{\prime}$ for all $i$, the epsilon stable sets are also those that are stochastically stable. 
Proof. See Appendix 2.9.4

Proposition 2 is a formal statement of the findings mentioned in the conceptual example above. It shows that if the probability distribution over the action set is uniform then there is an equivalence between epsilon and stochastically stable sets so long as the mutation rates are state independent. When this equivalence exists, we say that stochastic stability is robust. If on the other hand these distributions are skewed, then the first necessary condition for robustness of stochastic stability is they are bounded away from zero and one. Even so however, only for some values of $\varepsilon_{i}$ for all $i$ does there exists the equivalence between stochastic and epsilon stability. The main conclusion here is then that when applying the mistakes model to any evolutionary setting, unless the probability distributions over the action set is known, caution must be taken when interpreting what stochastically stable sets actually imply.

In Proposition 2 we assumed that the mutation rates are state independent. The effect of relaxing this assumption can equivalently be achieved by appropriately choosing the structure of probability distributions over the action set. In the literature, it is commonly assumed that the mutation rates are identical for all players and states. In this regard, let $\varepsilon_{i}=\exp (-\beta)$ for all players and states, such that $\varepsilon \rightarrow 0$ is equivalent to $\beta \rightarrow \infty$. For $\beta$ sufficiently large, we then have

$$
Q\left(\Omega, \Omega^{\prime}\right) \approx r\left(\Omega, \partial \Omega^{\prime}\right) \beta-r\left(\Omega, \partial \Omega^{\prime}\right) \ln \mathcal{P}_{A}\left(x_{\Omega^{\prime}}, \Omega\right)
$$

where $\mathcal{P}_{A}\left(x_{\Omega^{\prime}}, \Omega\right)=\frac{1}{N} \sum_{i=1}^{N} \mathcal{P}_{i}\left(x_{\Omega^{\prime}}, \Omega\right)$. Similarly,

$$
V\left(\Omega, \Omega^{\prime}\right)=\lim _{\beta \rightarrow \infty} \frac{1}{\beta} r\left(\Omega, \partial \Omega^{\prime}\right) \beta-r\left(\Omega, \partial \Omega^{\prime}\right) \ln \mathcal{P}_{A}\left(x_{\Omega^{\prime}}, \Omega\right)=r\left(\Omega, \partial \Omega^{\prime}\right)
$$

Such that $V(\Omega)=r_{\max }(\Omega)-r_{\min }$, where $r_{\min }(\Omega)=\min _{\Omega^{\prime} \neq \Omega} r\left(\Omega, \Omega^{\prime}\right)$ and $r_{\max }(\Omega)=$ $\max _{\Omega^{\prime} \neq \Omega} r\left(\Omega^{\prime}, \Omega\right)$ are the radius and co-radius of $\Omega$ according to Ellison [38]. Implying that the stochastically stable states are those with the minimum co-radius and maximum radius. In $2 \times 2$ coordination games, these are precisely states in which players play the risk-dominant strategies. Beyond $2 \times 2$ games however, riskdominant strategies are not necessarily stochastically stable (see Young [113] for a counter example). There is no guarantee however that risk-dominant strategies will be epsilon stable. It all depends on the specifications on the probability distributions over the action sets as illustrated above. In other words, stochastic stability is generally not robust under the mistakes model. 


\subsection{MULTINOMIAL LOGIT MODEL}

This section analyses the application of Theorem 1 and Corollary 1 to the multinomial logit model. Multinomial logit probabilities are a special case of perturbed best-response dynamics in which payoff errors are independent and extreme value distributed. The aim is to identify the states hence strategies that are typically epsilon and stochastically stable, and the robustness of stochastic stability.

Given the action set $X=\{a, b, c, \cdots\}$ of size $m$ and the set of errors $\varepsilon=\left\{\varepsilon_{a}, \varepsilon_{b}, \varepsilon_{c}, \cdots\right\}$. Each $\varepsilon_{x}$ is extreme value distributed, that is

$$
\mathbb{P}\left(\varepsilon_{x} \leq u\right)=\exp (\exp (-\beta u+\gamma))
$$

where $\gamma$ is Euler's constant and $\beta$ is the parameter that measures the level of noise. The smaller $\beta$ the larger the noise level and as $\beta$ tends to infinity the noise level tends to zero. Let $\Delta^{a}=\left[\delta_{b c}\right]$ where

$$
\delta_{b c}= \begin{cases}-1 & \text { if } b=c \\ 1 & \text { if } b=a \\ 0 & \text { otherwise }\end{cases}
$$

Such that $\varepsilon_{a b}=\left[\Delta^{a^{T}} \varepsilon\right]_{b}=\varepsilon_{a}-\varepsilon_{b}$. Similarly, if we write

$$
\mathbf{U}_{i}(\mathbf{x})=\left(U_{i}(a, \mathbf{x}), U_{i}(b, \mathbf{x}), U_{i}(c, \mathbf{x}), \cdots\right),
$$

then $\left[\Delta^{a^{T}} \mathbf{U}_{i}(\mathbf{x})\right]_{b}=U_{i}(a, \mathbf{x})-U_{i}(b, \mathbf{x})$. The conditional probability in (8) can equally be written as

$$
\mathbb{P}\left(\bigcap_{b \neq a}\left\{U_{i}\left(a, \mathbf{x}_{-i}\right)+\varepsilon_{a} \geq U_{i}\left(b, \mathbf{x}_{-i}\right)+\varepsilon_{b}\right\} ; \varepsilon_{a}\right)=\mathbb{P}\left(\Delta^{a^{T}} \varepsilon \leq \Delta^{a^{T}} \mathbf{U}_{i}(\mathbf{x}) ; \varepsilon_{a}\right)
$$

Following Anderson et al. [10, Section 2.6], (22) and (24) lead to the choice probabilities of the form

$$
\mathbb{P}_{i}(a, \mathbf{x})=\frac{\exp \left(\beta U_{i}\left(a, \mathbf{x}_{-i}\right)\right)}{\sum_{b \in X} \exp \left(\beta U_{i}\left(b, \mathbf{x}_{-i}\right)\right)}=\frac{1}{1+\sum_{b \neq a} \exp \left(-\beta\left[\Delta^{a^{T}} \mathbf{U}_{i}(\mathbf{x})\right]_{b}\right)}
$$

Now, let $\left[\Delta^{a^{T}} \mathbf{U}_{i}(\mathbf{x})\right]_{\min }=\min _{b \neq a}\left[\Delta^{a^{T}} \mathbf{U}_{i}(\mathbf{x})\right]_{b}$ and $U_{i}(b, \Omega)$ be the payoff of playing $a$ when the state is some state of the closed communication class $\Omega$. Generally $\left[\Delta^{a^{T}} \mathbf{U}_{i}(\Omega)\right]_{b} \leq 0$ otherwise $\Omega$ would not be a closed communication class. Hence, for $\beta$ sufficiently large $1+\sum_{b \neq a} \exp \left(-\beta\left[\Delta^{a^{T}} \mathbf{U}_{i}(\Omega)\right]_{b}\right) \approx \exp \left(-\beta\left[\Delta^{a^{T}} \mathbf{U}_{i}(\Omega)\right]_{\min }\right)$. Such that $\mathbb{P}_{i}(a, \mathbf{x}) \approx \exp \left(\beta\left[\Delta^{a^{T}} \mathbf{U}_{i}(\Omega)\right]_{\min }\right)$. It then follows that for all $i \in \mathcal{N}$

$$
c\left(\Omega, \partial \Omega^{\prime}\right)=-r\left(\Omega, \partial \Omega^{\prime}\right) \ln \left(\frac{1}{N} \sum_{i=1}^{N} \exp \left\{\beta\left[\left(\Delta^{x} \Omega^{\prime}\right)^{T} \mathbf{U}_{i}(\Omega)\right]_{\min }\right\}\right)
$$


LEMmA 3: Let $\left[\left(\Delta^{x_{\Omega^{\prime}}}\right)^{T} \mathbf{U}_{\max }(\Omega)\right]_{\min }=\max _{i \in \mathcal{N}}\left[\left(\Delta^{x_{\Omega^{\prime}}}\right)^{T} \mathbf{U}_{i}(\Omega)\right]_{\min }$. Given (26), $Q\left(\Omega, \partial \Omega^{\prime}\right)$ and $V\left(\Omega, \partial \Omega^{\prime}\right)$ are respectively given by

$$
\begin{gathered}
Q\left(\Omega, \partial \Omega^{\prime}\right)=-r\left(\Omega, \partial \Omega^{\prime}\right) \ln \left(\frac{1}{N} \sum_{i=1}^{N} \exp \left\{\beta\left[\left(\Delta^{x} \Omega^{\prime}\right)^{T} \mathbf{U}_{i}(\Omega)\right]_{\min }\right\}\right)+F\left(r\left(\Omega, \partial \Omega^{\prime}\right)\right) \\
V\left(\Omega, \partial \Omega^{\prime}\right) \leq-r\left(\Omega, \partial \Omega^{\prime}\right)\left[\left(\Delta^{x} \Omega^{\prime}\right)^{T} \mathbf{U}_{\max }(\Omega)\right]_{\min }
\end{gathered}
$$

Proof. The proof for (27) follows directly from (26). For the case of (29) we have that,

$$
\begin{aligned}
\sum_{i=1}^{N} \exp \left\{\beta\left[\left(\Delta^{x} \Omega^{\prime}\right)^{T} \mathbf{U}_{i}(\Omega)\right]_{\min }\right\} & \leq \sum_{i=1}^{N} \exp \left\{\beta\left[\left(\Delta^{x_{\Omega^{\prime}}}\right)^{T} \mathbf{U}_{\max }(\Omega)\right]_{\min }\right\} \\
& =N \exp \left\{\beta\left[\left(\Delta^{x} \Omega^{\prime}\right)^{T} \mathbf{U}_{\max }(\Omega)\right]_{\min }\right\}
\end{aligned}
$$

Such that

$$
\begin{aligned}
V\left(\Omega, \partial \Omega^{\prime}\right) & =\lim _{\beta \rightarrow \infty}-\frac{1}{\beta} r\left(\Omega, \partial \Omega^{\prime}\right) \ln \left(\frac{1}{N} \sum_{i=1}^{N} \exp \left\{\beta\left[\left(\Delta^{x} \Omega^{\prime}\right)^{T} \mathbf{U}_{i}(\Omega)\right]_{\min }\right\}\right) \\
& \leq-r\left(\Omega, \partial \Omega^{\prime}\right)\left[\left(\Delta^{x^{\prime}}\right)^{T} \mathbf{U}_{\max }(\Omega)\right]_{\min }
\end{aligned}
$$

The following Proposition states the result on robustness of stochastic stability under multinomial logit model.

Proposition 3: There exists an equivalence between stochastically stable and epsilon stable sets under the multinomial logit model.

Proof. From Lemma 3, we see that the only relevant quantities in the computational process of both epsilon and stochastically stable sets are $r\left(\Omega, \partial \Omega^{\prime}\right)\left[\left(\Delta^{x} \Omega^{\prime}\right)^{T} \mathbf{U}_{i}(\Omega)\right]_{\min }$. In fact, since $-0.7 \leq F\left(r\left(\Omega, \partial \Omega^{\prime}\right)\right) \leq 0$, then for $\beta$ sufficiently large

$$
Q\left(\Omega, \partial \Omega^{\prime}\right) \leq-r\left(\Omega, \partial \Omega^{\prime}\right)\left[\left(\Delta^{x_{\Omega^{\prime}}}\right)^{T} \mathbf{U}_{\max }(\Omega)\right]_{\min } \beta
$$

such that $-r\left(\Omega, \partial \Omega^{\prime}\right)\left[\left(\Delta^{x_{\Omega^{\prime}}}\right)^{T} \mathbf{U}_{\max }(\Omega)\right]_{\min }$ are the only relevant quantities for both epsilon and stochastic stability.

Proposition 3 directly implies the robustness of stochastic stability under multinomial logit model. This result is contrary to that of the mistakes model above. From the expressions of $Q\left(\Omega, \Omega^{\prime}\right)$ and $V\left(\Omega, \Omega^{\prime}\right)$ above, it is clear that the only relevant quantities in the computations of both epsilon and stochastically stable sets 
is $r\left(\Omega, \partial \Omega^{\prime}\right)\left[\left(\Delta^{x} \Omega^{\prime}\right)^{T} \mathbf{U}_{i}(\Omega)\right]_{\text {min. }}$. Unlike in the usual computation of stochastic stability where only $r\left(\Omega, \partial \Omega^{\prime}\right)$ are relevant, here we find an additional component $\left[\left(\Delta^{x} \Omega^{\prime}\right)^{T} \mathbf{U}_{i}(\Omega)\right]_{\text {min }}$, the payoff loss associated with the transition $\Omega \rightarrow \Omega^{\prime}$. Implying that the larger the payoff loss, the larger the cost of such a transition. Since risk-dominant actions are usually the ones that exhibit the largest payoff losses, they should then be the most likely to be selected in the long-run under multinomial logit dynamics. This has been shown to be the case for $2 \times 2$ matrix games (e.g Maruta [71], Blume [20], Okada and Tercieux [89] among others). Beyond $2 \times 2$ games however, there is no guarantee that dominant actions are selected (see Example 2). In comparison to the mistakes model, dominant actions are for sure more likely to be selected under multinomial logit models.

In symmetric games, $\mathbb{P}_{i}\left(x_{\Omega^{\prime}}, \Omega\right)$ is identical for all players, such that

$$
V\left(\Omega, \Omega^{\prime}\right)=-r\left(\Omega, \partial \Omega^{\prime}\right)\left[\left(\Delta^{x} \Omega^{\prime}\right)^{T} \mathbf{U}(\Omega)\right]_{\min }
$$

The stochastic potential for any given tree $g$ then becomes

$$
\mathscr{S}_{\mathcal{S}}(\Omega)=\min _{g \in G(\{\Omega\})} \sum_{\left(\Omega_{j}, \Omega_{\kappa}\right) \in g}-r\left(\Omega, \partial \Omega^{\prime}\right)\left[\left(\Delta^{x_{\Omega^{\prime}}}\right)^{T} \mathbf{U}(\Omega)\right]_{\min }
$$

The quantities on the right hand side of (31) are readily computable from the payoffs. Note also that the expression of the stochastic potential that results from the above measures such as (31), is equivalent to the measure of waste of a tree $g$ according to Alós-Ferrer and Netzer [7] when players follow simultaneous revision protocol.

\subsection{EXAMPLES}

The following examples provide an illustration of how to compute epsilon stable sets based on the algorithmic steps above. They also act as applications of the results of Theorem 1 and Corollary 1 to both mistakes and multnomial logit models. The example adopted from Young [113] is used to show the case in which risk-dominant actions are selected under multinomial logit but not under mistakes model. The second example shows that risk-dominant actions need not be selected under multinomial logit models.

Example 1. Consider the $3 \times 3$ games of Table 3 played by a sufficiently large number of players.

(i) If players follow the mistakes model with $\varepsilon=\exp (-\beta)$ and $\mathcal{P}=\frac{1}{m}$, then $\mathbf{b}$ is both epsilon and stochastically stable. 
(ii) If players follow the mistakes model with $\varepsilon=\exp (-\beta)$ and $\mathcal{P}(a, \mathbf{x})=\frac{6}{21}, \mathcal{P}(b, \mathbf{x})=$ $\frac{7}{21}, \mathcal{P}(c, \mathbf{x})=\frac{8}{21}$ for all $i$ and $\mathbf{x}$, then for all $\beta<1.6, \mathbf{c}$ is epsilon stable and for all $\beta>1.6, \mathbf{b}$ is both epsilon and stochastically stable.

(iii) If players follow the multinomial logit model, then $\mathbf{c}$ is both epsilon and stochastically stable.

Table 3: For any pair of players the profile $(c, c)$ is risk-dominant.

a

\begin{tabular}{|c|c|c|}
\hline 6,6 & 0,5 & 0,0 \\
\hline 5,0 & 7,7 & 5,5 \\
\hline 0,0 & 5,5 & 8,8 \\
\hline
\end{tabular}

Proof. For the game in table 3, if players are uniformly and randomly matched then there are three singleton closed communication classes $\Omega=\{\mathbf{a}, \mathbf{b}, \mathbf{c}\}$, the all $a$, all $b$ and all $c$ states respectively. The second step is to calculate for the quantities $r\left(\Omega, \partial \Omega^{\prime}\right)$ for each pair of limit states. For the pair $(\mathbf{a}, \mathbf{b})$ for example we have 5

$$
\begin{array}{r}
r(\mathbf{a}, \partial \mathbf{b})=\max \left\{\frac{U(a, a)-U(b, a)}{(U(a, a)-U(b, a))+(U(b, b)-U(a, b))},\right. \\
\left.\frac{U(a, a)-U(b, a)}{(U(c, a)-U(b, a))+(U(b, b)-U(c, b))}\right\}
\end{array}
$$

where $U(x, y)$ is the payoff derived from playing $x$ when the opponent plays $y$. By substituting the payoffs from Table 3 it follows that $r(\mathbf{a}, \partial \mathbf{b})=1 / 8$. A similar argument applies to all directed relations between all pairs of limit sets yielding $r(\mathbf{a}, \partial \mathbf{b})=\frac{1}{8}, r(\mathbf{b}, \partial \mathbf{a})=\frac{7}{8}, r(\mathbf{b}, \partial \mathbf{c})=\frac{2}{5}, r(\mathbf{c}, \partial \mathbf{b})=\frac{3}{8}, r(\mathbf{a}, \partial \mathbf{c})=\frac{5}{8}, r(\mathbf{c}, \partial \mathbf{a})=\frac{5}{6}$. Note for example that for the the direct transition $\mathbf{c} \rightarrow \mathbf{b}$ it requires $\frac{3}{5}$ players to simultaneously switch to play $b$, the shortest indirect transition is for at least $\frac{3}{8}$ to play $a$.

$$
\begin{aligned}
& r(\mathbf{a}, \partial \mathbf{b}) U(b, b)+(1-r(\mathbf{a}, \partial \mathbf{b})) U(b, a)>r(\mathbf{a}, \partial \mathbf{b}) U(a, b)+(1-r(\mathbf{a}, \partial \mathbf{b})) U(a, a) \\
& r(\mathbf{a}, \partial \mathbf{b}) U(b, b)+(1-r(\mathbf{a}, \partial \mathbf{b})) U(b, a)>r(\mathbf{a}, \partial \mathbf{b}) U(c, b)+(1-r(\mathbf{a}, \partial \mathbf{b})) U(c, a)
\end{aligned}
$$


(i) Each $V\left(\Omega, \Omega^{\prime}\right)$ is equivalent to $r\left(\Omega, \partial \Omega^{\prime}\right)$ and each $Q\left(\Omega, \Omega^{\prime}\right)=r\left(\Omega, \partial \Omega^{\prime}\right)(\beta-$ $\left.\ln 3^{-1}\right)+F\left(r\left(\Omega, \partial \Omega^{\prime}\right)\right)$. We then have $Q(\mathbf{a}, \mathbf{b})=\frac{1}{8} \beta-0.24, Q(\mathbf{b}, \mathbf{a})=\frac{7}{8} \beta+0.59$, $Q(\mathbf{b}, \mathbf{c})=\frac{2}{5} \beta-0.23, Q(\mathbf{c}, \mathbf{b})=\frac{3}{8} \beta-0.25, Q(\mathbf{a}, \mathbf{c})=\frac{5}{8} \beta-0.12, Q(\mathbf{c}, \mathbf{a})=\frac{5}{6} \beta+$ 0.47 .

The above quantities yield $V(\mathbf{a})=r(\mathbf{b}, \partial \mathbf{a})-r(\mathbf{a}, \partial \mathbf{b})=\frac{7}{8}-\frac{1}{8}>0, V(\mathbf{b})=$ $r(\mathbf{c}, \partial \mathbf{b})-r(\mathbf{b}, \partial \mathbf{c})=\frac{3}{8}-\frac{2}{5}<0$ and $V(\mathbf{c})=r(\mathbf{a}, \partial \mathbf{c})-r(\mathbf{c}, \partial \mathbf{b})=\frac{5}{8}-\frac{3}{8}>0$. Similarly, $Q(\mathbf{a})=\frac{3}{4} \beta+0.83>0, Q(\mathbf{b})=-\frac{1}{40} \beta-0.02<0$ and $Q(\mathbf{c})=\frac{3}{20} \beta+$ $0.12>0$. Implying that $\mathbf{b}$ is both the epsilon and stochastically stable state.

(ii) For $\mathcal{P}(a, \mathbf{x})=\frac{6}{21}, \mathcal{P}(b, \mathbf{x})=\frac{7}{21}, \mathcal{P}(c, \mathbf{x})=\frac{8}{21} ; Q(\mathbf{a}, \mathbf{b})=\frac{1}{8} \beta-0.2, Q(\mathbf{b}, \mathbf{a})=\frac{7}{8} \beta+$ $0.72, Q(\mathbf{b}, \mathbf{c})=\frac{2}{5} \beta-0.29, Q(\mathbf{c}, \mathbf{b})=\frac{3}{8} \beta-0.25, Q(\mathbf{a}, \mathbf{c})=\frac{5}{8} \beta-0.06, Q(\mathbf{c}, \mathbf{a})=$ $\frac{5}{6} \beta+0.59$. When $\beta<1.6, Q(\mathbf{a})>0, Q(\mathbf{b})=-\frac{1}{40} \beta+0.04>0$ and $Q(\mathbf{c})=$ $\frac{1}{40} \beta-0.04<0$. But when $\beta>1.6, Q(\mathbf{a})>0, Q(\mathbf{b})<0$ and $Q(\mathbf{c})>0$.

(iii) We need to derive values for the quantities $\left[\left(\Delta^{x} \Omega^{\prime}\right)^{T} \mathbf{U}(\Omega)\right]_{\min }$ for each pair $\left(\Omega, \Omega^{\prime}\right)$. Note that we write $\left[\left(\Delta^{x} \Omega^{\prime}\right)^{T} \mathbf{U}(\Omega)\right]_{\min }$ for all players since the game is symmetric, in which case the error probabilities are identical. Consider $(\mathbf{a}, \mathbf{b})$ for example, $\left[\left(\Delta^{b}\right)^{T} \mathbf{U}(\mathbf{a})\right]_{a}=-1,\left[\left(\Delta^{b}\right)^{T} \mathbf{U}(\mathbf{a})\right]_{b}=0,\left[\left(\Delta^{b}\right)^{T} \mathbf{U}(\mathbf{a})\right]_{c}=5$. Implying that $\left[\left(\Delta^{b}\right)^{T} \mathbf{U}(\mathbf{a})\right]_{\min }=-1$. Similarly for $(\mathbf{c}, \mathbf{b})$, since $a$ is the action that is played for the transition $\mathbf{c} \rightarrow \partial \mathbf{b}$ to occur, we then have $\left[\left(\Delta^{a}\right)^{T} \mathbf{U}(\mathbf{c})\right]_{a}=0$, $\left[\left(\Delta^{a}\right)^{T} \mathbf{U}(\mathbf{c})\right]_{b}=-5,\left[\left(\Delta^{a}\right)^{T} \mathbf{U}(\mathbf{c})\right]_{c}=-8$. Implying that $\left[\left(\Delta^{a}\right)^{T} \mathbf{U}(\mathbf{c})\right]_{\min }=-8$. For the remainder of the pairwise relations we have, $\left[\left(\Delta^{x_{a}}\right)^{T} \mathbf{U}(\mathbf{b})\right]_{\min }=-7$, $\left[\left(\Delta^{x_{\mathfrak{c}}}\right)^{T} \mathbf{U}(\mathbf{b})\right]_{\min }=-2,\left[\left(\Delta^{x_{\mathbf{c}}}\right)^{T} \mathbf{U}(\mathbf{a})\right]_{\min }=-6$ and $\left[\left(\Delta^{x_{\mathbf{a}}}\right)^{T} \mathbf{U}(\mathbf{c})\right]_{\min }=-8$.

For $\beta$ sufficiently large, we can approximate

$$
Q\left(\Omega, \Omega^{\prime}\right) \approx-r\left(\Omega, \partial \Omega^{\prime}\right)\left[\left(\Delta^{x} \Omega^{\prime}\right)^{T} \mathbf{U}_{i}(\Omega)\right]_{\min } \beta
$$

. We then have $Q(\mathbf{a}, \mathbf{b}) \approx 0.13 \beta, Q(\mathbf{b}, \mathbf{a})=6.13 \beta, Q(\mathbf{b}, \mathbf{c})=0.8 \beta, Q(\mathbf{c}, \mathbf{b})=3 \beta$, $Q(\mathbf{a}, \mathbf{c})=3.75 \beta, Q(\mathbf{c}, \mathbf{a})=6.67 \beta$. See Figure 1 for the graphical representation of costs of transitions.

Hence $Q(\mathbf{a})=6.13 \beta-0.13 \beta>0, Q(\mathbf{b})=3 \beta-0.8 \beta>0$ and $Q(\mathbf{c})=0.8 \beta-$ $3 \beta>0$. Implying that $\mathbf{c}$ is both the epsilon and stochastically stable state. A similar result obtains by using the tree algorithm. From Figure 1 the minimum cost tree is $\{\mathbf{a} \rightarrow \mathbf{b} \rightarrow \mathbf{c}\}$ with the stochastic potential of $\mathscr{S}(\mathbf{c})=0.93 \beta$.

Example 2. Consider the $3 \times 3$ games of Table 4 played by a sufficiently large number of players. If players follow the multinomial logit model, then $\mathbf{b}$ is both epsilon and stochastically stable. 


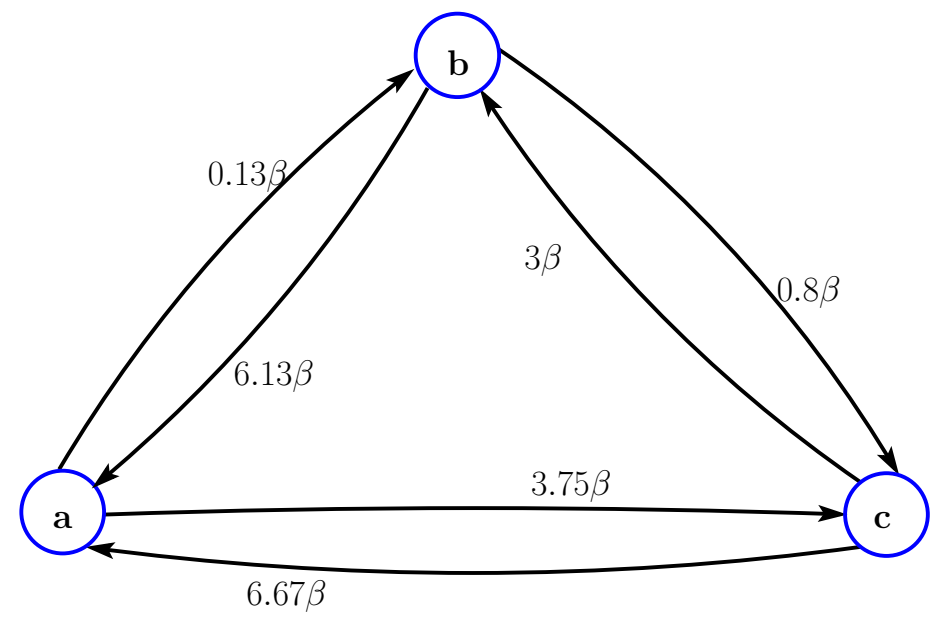

Figure 1: The costs of transitions between closed communication classes for the multinomial logit model.

Proof. The limit states are $\mathbf{b}$ and $\mathbf{c}$. Following similar steps as in the proof of Example 3 above, we find that $r(\mathbf{b}, \mathbf{c})=\frac{3}{6.5}$ and $r(\mathbf{c}, \mathbf{b})=\frac{3.5}{10.5}$. The later follows from the fact that for the transition $\mathbf{c} \rightarrow \partial \mathbf{b}$ to occur, at least $\frac{3.5}{10.5}$ of the players should play action $a$. Similarly $\left[\left(\Delta^{x_{\mathfrak{c}}}\right)^{T} \mathbf{U}(\mathbf{b})\right]_{\min }=-3$ and $\left[\left(\Delta^{x_{\mathfrak{b}}}\right)^{T} \mathbf{U}(\mathbf{c})\right]_{\min }=-1$, and hence $V(\mathbf{b}, \mathbf{c})=\frac{9}{6.5}$ and $V(\mathbf{c}, \mathbf{b})=\frac{3.5}{10.5}$. Consequently $V(\mathbf{b})<0$ and $V(\mathbf{c})>0$. Implying that $\mathbf{b}$ is the stochastically stable state.

Examples 3 and 2 act to illustrate the three aspects of our main findings. The first is that for the mistakes model, provided the probability distributions over actions are bounded then there always exists the level of noise below which stochastic stability is robust. For the game 4 , if the probability distribution over actions is proportional to the equilibrium payoffs, then for all values of $\beta>1.6$ or equivalently $\varepsilon<0.2$ stochastic stability is robust to noise levels. The second aspect is that dominant strategies are more likely to be selected under multinomial logit model than mistakes model. Lastly, in Example 2, $c$ is the risk-dominant action but the stochastically stable state is $\mathbf{b}$. Implying that risk-dominant actions need not be selected in general even under multinomial logit models. Admittedly however, there are not many (if they exist) real world games that are representative of Example 2. 
Table 4: For any pair of players the profile $(c, c)$ is risk-dominant.

a

b

C

\begin{tabular}{|c|c|c|}
\hline \multicolumn{1}{c|}{$\mathrm{a}$} & $\mathrm{b}$ & $\mathrm{c}$ \\
\hline 0,0 & 7,7 & 6,0 \\
\hline 7,7 & 8,8 & 5,5 \\
\hline 0,6 & 5,5 & $8.5,8.5$ \\
\hline
\end{tabular}

\subsection{CONCLUSION}

Stochastic evolutionary models have been and still are an important approach in game theory. They are most appealing in their remarkable ability to select among multiple equilibria and as a means of modeling repeated interactions and experimentation among agents. This Chapter developed a general framework for noisy stochastic evolutionary dynamics with the objective of circumventing some of the main limitations and criticisms surrounding such models. The first main contribution of this Chapter has been to define the concept of epsilon stability as a more general solution concept than the commonly used notion of stochastic stability. We then provided an efficient algorithm for computing epsilon and stochastically stable sets that is based on fairly fundamental measures.

We have been able to establish analytical results concerning robustness of stochastic stability to noise levels by relating it to epsilon stability. The question of robustness is only critical for the mistakes models. We show that provided the probability distribution over the strategy set is bounded, then there exists the level of noise below which stochastic stability is robust. If the probability distribution over strategies is uniform then stochastic stability is robust to all levels of noise. Under multinomial logit models, stochastic stability is robust to noise.

Finally, application of the computational algorithm shows that dominant strategies are more likely to be selected under multinomial logit model than the mistakes model. Dominant strategies however need not be selected in general. We conjecture that the results concerning robustness and selection of risk-dominant strategies for multinomial logit model directly extend to multinomial probit models that are studied in Myatt and Wallace [82] and Dokumac1 and Sandholm [35]. This is based on the fact that in both multinomial logit and probit models, the probabilities of errors are an exponential functions of payoff losses associated with the transitions and that 
it is this property that influences robustness and selection of dominant strategies. A proof of this conjecture however requires a more rigorous analysis that we postpone as a future research agenda.

\subsection{APPENDIX}

\subsubsection{Proof of Lemma 5}

The proof follows by considering the properties of the reduced chain $\left(\tilde{\Omega}, \tilde{P}_{\varepsilon}\right)$ with stationary distribution $\tilde{\pi}$. The formal definition of $\left(\tilde{\Omega}, \tilde{P}_{\mathcal{\varepsilon}}\right)$ is as follows (a generalization of the collapsed Markov chain in Aldous and Fill [4, Chapter 2]):

$$
\begin{aligned}
\tilde{P}_{\mathcal{E}}(\mathbf{x}, \mathbf{y}) & =P_{\mathcal{\varepsilon}}(\mathbf{x}, \mathbf{y}) \\
\tilde{P}_{\mathcal{E}}(\mathbf{y}, \tilde{\Omega}) & =\sum_{\mathbf{x} \in \tilde{\Omega}} P_{\mathcal{E}}(\mathbf{y}, \mathbf{x}) \\
\tilde{P}_{\mathcal{E}}(\tilde{\Omega}, \mathbf{y}) & =\frac{1}{\pi(\tilde{\Omega})} \sum_{\mathbf{x} \in \tilde{\Omega}} \pi(\mathbf{x}) P_{\mathcal{\varepsilon}}(\mathbf{x}, \mathbf{y}), \\
\tilde{P}_{\mathcal{E}}\left(\tilde{\Omega}, \tilde{\Omega}^{\prime}\right) & =\frac{1}{\pi(\tilde{\Omega})} \sum_{\mathbf{x} \in \tilde{\Omega}} \sum_{\mathbf{y} \in \tilde{\Omega}^{\prime}} \pi(\mathbf{x}) P_{\mathcal{\varepsilon}}(\mathbf{x}, \mathbf{y})
\end{aligned}
$$

The following lemma is an immediate consequence of the above definition of a collapsed Markov chain.

LeMmA 4: Let $\pi$ and $\tilde{\pi}$ be the stationary distributions of $\left(\mathbf{X}, P_{\varepsilon}\right)$ and $\left(\tilde{\Omega}, \tilde{P}_{\varepsilon}\right)$ respectively. Then for any $\tilde{\Omega} \in \tilde{\Omega}, \tilde{\pi}(\tilde{\Omega})=\pi(\tilde{\Omega})=\sum_{\mathbf{x} \in \tilde{\Omega}} \pi(\mathbf{x})$

Proof. Note that $\pi(\tilde{\Omega})=\sum_{\mathbf{x} \in \tilde{\Omega}} \pi(\mathbf{x})$. Let $\# \mathbf{X}$ and $\# \tilde{\Omega}$ be the cardinality of $\mathbf{X}$ and $\tilde{\Omega}$ respectively. Define an event matrix $\mathcal{E}$ as an $\# \mathbf{X} \times \# \tilde{\Omega}$ matrix whose entries take on a value one if a state $\mathbf{x} \in \Omega$ belongs to $\tilde{\Omega} \in \tilde{\Omega}$ and zero otherwise. Denote by $\mathcal{E}_{\tilde{\Omega}}$ for the $\tilde{\Omega}^{\text {th }}$ column of $\mathcal{E}$. It then follows that $\tilde{P}_{\mathcal{E}} \mathcal{E}=\mathcal{E} \tilde{P}_{\mathcal{E}}$, and that

$$
\pi(\tilde{\Omega})=\pi \mathcal{E}_{\tilde{\Omega}} \quad \forall \tilde{\Omega} \in \tilde{\Omega}
$$

Consequently, $\pi \mathcal{E}=\pi \tilde{P}_{\mathcal{E}} \mathcal{E}=\pi \mathcal{E} \tilde{P}_{\mathcal{E}}$. Implying that $\pi \mathcal{E}$ is the stationary distribution of $\tilde{P}_{\mathcal{E}}$, hence $\tilde{\pi}=\pi \mathcal{E}$.

Now, since the reduced chain is also irreducible just like the original chain, for any pair of subsets $\tilde{\Omega}, \tilde{\Omega}^{\prime} \in \tilde{\Omega}$ with $U_{\tilde{\Omega} \tilde{\Omega}^{\prime}}=\tilde{\Omega} \cup \tilde{\Omega}^{\prime}$ and $U_{\tilde{\Omega} \tilde{\Omega}^{\prime}}^{c}$ its complement.

$$
\begin{aligned}
& \pi_{\mathcal{E}}(\tilde{\Omega}) \tilde{P}_{\mathcal{E}}(\tilde{\Omega}, \tilde{\Omega})+\pi_{\mathcal{E}}\left(\tilde{\Omega}^{\prime}\right) \tilde{P}_{\mathcal{E}}\left(\tilde{\Omega}^{\prime}, \tilde{\Omega}\right)+\pi_{\mathcal{\varepsilon}}\left(U_{\tilde{\Omega} \tilde{\Omega}^{\prime}}^{c}\right) \tilde{P}_{\mathcal{E}}\left(U_{\tilde{\Omega} \tilde{\Omega}^{\prime \prime}}^{c} \tilde{\Omega}\right)=\pi_{\mathcal{\varepsilon}}(\tilde{\Omega}) \\
& \pi_{\varepsilon}(\tilde{\Omega})\left(1-\tilde{P}_{\mathcal{\varepsilon}}(\tilde{\Omega}, \tilde{\Omega})\right)=\pi_{\varepsilon}\left(\tilde{\Omega}^{\prime}\right) \tilde{P}_{\mathcal{\varepsilon}}\left(\tilde{\Omega}^{\prime}, \tilde{\Omega}\right)+\pi_{\varepsilon}\left(U_{\tilde{\Omega} \tilde{\Omega}^{\prime}}^{c}\right) \tilde{P}_{\mathcal{\varepsilon}}\left(U_{\tilde{\Omega} \tilde{\Omega}^{\prime \prime}}^{c}, \tilde{\Omega}\right)
\end{aligned}
$$




$$
\frac{\pi_{\varepsilon}\left(\tilde{\Omega}^{\prime}\right)}{\pi_{\varepsilon}(\tilde{\Omega})}=\frac{1-\tilde{P}_{\mathcal{\varepsilon}}(\tilde{\Omega}, \tilde{\Omega})}{\tilde{P}_{\varepsilon}\left(\tilde{\Omega}^{\prime}, \tilde{\Omega}\right)}-\frac{\pi_{\varepsilon}\left(U_{\tilde{\Omega}}^{c} \tilde{\Omega}^{\prime}\right)}{\pi_{\varepsilon}(\tilde{\Omega})} \frac{\tilde{P}_{\mathcal{E}}\left(U_{\tilde{\Omega} \tilde{\Omega}^{\prime \prime}}^{c}, \tilde{\Omega}\right)}{\tilde{P}_{\mathcal{\varepsilon}}\left(\tilde{\Omega}^{\prime}, \tilde{\Omega}\right)}
$$

By substituting for $1-\tilde{P}_{\mathcal{E}}(\tilde{\Omega}, \tilde{\Omega})=\tilde{P}_{\mathcal{E}}\left(\tilde{\Omega}, \tilde{\Omega}^{c}\right)$, we then have

$$
\frac{\pi_{\varepsilon}\left(\tilde{\Omega}^{\prime}\right)}{\pi_{\varepsilon}(\tilde{\Omega})} \leq \frac{\tilde{P}_{\varepsilon}\left(\tilde{\Omega}, \tilde{\Omega}^{c}\right)}{\tilde{P}_{\varepsilon}\left(\tilde{\Omega}^{\prime}, \tilde{\Omega}\right)}
$$

Since we are considering simultaneous revision protocol, the transition $\tilde{\Omega} \rightarrow \tilde{\Omega}^{\prime}$ can equivalently be treated as a step-by-step transition $\partial \Omega \rightarrow \Omega \rightarrow \partial \Omega^{\prime} \rightarrow \Omega^{\prime}$. Such that

$$
\tilde{P}_{\mathcal{E}}\left(\tilde{\Omega}, \tilde{\Omega}^{\prime}\right) \equiv P_{\mathcal{E}}(\partial \Omega, \Omega) P_{\mathcal{E}}\left(\Omega, \partial \Omega^{\prime}\right) P_{\mathcal{E}}\left(\partial \Omega^{\prime}, \Omega^{\prime}\right)
$$

The above argument follows from the fact that under simultaneous revision protocol, at least $R\left(\Omega, \partial \Omega^{\prime}\right)$ players must simultaneous make a mistake for the transition $\Omega \rightarrow$ $\partial \Omega^{\prime}$ to occur. Meaning that the process spends most time in $\Omega$ when in the basin of attraction $\tilde{\Omega}$. Under random matching, the transitions $\partial \Omega \rightarrow \Omega$ and $\partial \Omega^{\prime} \rightarrow \Omega^{\prime}$ occur more rapidly compared to $\Omega \rightarrow \partial \Omega^{\prime}$. Hence

$$
\tilde{P}_{\mathcal{\varepsilon}}\left(\tilde{\Omega}, \tilde{\Omega}^{\prime}\right) \approx P_{\mathcal{\varepsilon}}\left(\Omega, \Omega^{\prime}\right) \approx P_{\mathcal{\varepsilon}}\left(\Omega, \partial \Omega^{\prime}\right)
$$

\subsubsection{Proof of Proposition 4}

The rest of the proof follows a constructive approach. Consider the following experiment. Pick a random profile $\left(\mathbb{P}_{1}\left(x_{\Omega^{\prime}}, \Omega\right), \cdots, \mathbb{P}_{N}\left(x_{\Omega^{\prime}}, \Omega\right)\right)$ from the distributions of $I_{1}, \cdots, I_{N}$, and from this profile pick a set $U \sim \operatorname{Bin}(N, \sigma)$, where $0 \leq \sigma \leq 1$ is an arbitrary parameter whose optimal value will be computed. Each $\mathbb{P}_{i}\left(x_{\Omega^{\prime}}, \Omega\right)$ is therefore in $U$ with probability $\sigma$.

Define an event $\forall_{i \in U} I_{i}=1$; that is all members of $U$ choose $x_{\Omega^{\prime}}$, and consequently $\mathbb{P}\left(\forall_{i \in U} I_{i}=1\right)$ is the probability that all $i \in U$ choose $x_{\Omega^{\prime}}$. From the partition theorem for sets, the following inequality holds

$$
\begin{aligned}
& \mathbb{P}\left(\sum_{i=1}^{N} I_{i} \geq r\left(\Omega, \partial \Omega^{\prime}\right) N\right) \mathbb{E}\left[\forall_{i \in U} I_{i}=1 \mid \sum_{i=1}^{N} I_{i} \geq r\left(\Omega, \partial \Omega^{\prime}\right) N\right] \leq \mathbb{E}\left[\forall_{i \in U} I_{i}=1\right] \\
& \leq K_{1} \mathbb{P}\left(\sum_{i=1}^{N} I_{i} \geq r\left(\Omega, \partial \Omega^{\prime}\right) N\right) \mathbb{E}\left[\forall_{i \in U} I_{i}=1 \mid \sum_{i=1}^{N} I_{i} \geq r\left(\Omega, \partial \Omega^{\prime}\right) N\right]
\end{aligned}
$$

where the expectations are taken over the realizations of $U$. Define the set

$$
\mathscr{F}_{r}=\{U: U \subseteq\{1, \cdots, N\}, \# U=r\}
$$


such that the cardinality of $\mathscr{F}_{r}, \# \mathscr{F}_{r}=\left(\begin{array}{c}N \\ r\end{array}\right)$, is a binomial coefficient. Since $\mathbb{P}\left(\forall_{i \in U} I_{i}=\right.$ $1)=\prod_{i \in U} \mathbb{P}_{i}\left(x_{\Omega^{\prime}}, \Omega\right)$, we then have

$$
\mathbb{E}\left[\forall_{i \in U} I_{i}=1\right]=\sum_{r=0}^{N} \sum_{U \in \mathscr{F}_{r}}\left(\sigma^{\# U}(1-\sigma)^{n-\# U} \prod_{i \in U} \mathbb{P}_{i}\left(x_{\Omega^{\prime}}, \Omega\right)\right)
$$

Using the following identity

$$
\sum_{r=0}^{N} \sum_{U \in \mathscr{F}_{r}}\left\{\left(\prod_{i \in U} \alpha_{i}\right)\left(\prod_{j \in U^{c}} \gamma_{j}\right)\right\}=\prod_{i=1}^{N}\left(\alpha_{i}+\gamma_{i}\right)
$$

equation (2.9.3) becomes

$$
\mathbb{E}\left[\forall_{i \in U} I_{i}=1\right]=\prod_{i=1}^{N}\left(\sigma \mathbb{P}_{i}\left(x_{\Omega^{\prime}}, \Omega\right)+1-\sigma\right)
$$

We can now employ the bounds for the difference between arithmetic and geometric means to place bounds on $\mathbb{E}\left[\forall_{i \in U} I_{i}=1\right]$. Given the vector $V=\left(p_{1}, \cdots, p_{N}\right)$ of individual probabilities, let $\mathbb{P}_{A}(V)$ and $\Pi_{A}(V)$ denote its arithmetic and geometric means respectively. Tighter bounds for the difference $\mathbb{P}_{A}(V)-\Pi_{A}(V)$ can be found in the literature but here, we make use of the weaker relation $0 \leq \mathbb{P}_{A}(V)-\Pi_{A}(V) \leq$ $\left(p_{\max }-p_{\min }\right)$, where $p_{\min }$ and $p_{\max }$ are the minimum and maximum entries of $V$. It then follows that there exists a constant $K_{A}$ such that

$$
K_{A}\left(\sigma \mathbb{P}_{A}\left(x_{\Omega^{\prime}}, \Omega\right)+1-\sigma\right)^{N} \leq \mathbb{E}\left[\forall_{i \in U} I_{i}=1\right] \leq\left(\sigma \mathbb{P}_{A}\left(x_{\Omega^{\prime}}, \Omega\right)+1-\sigma\right)^{N}
$$

where $\mathbb{P}_{A}\left(x_{\Omega^{\prime}}, \Omega\right)=\frac{1}{n} \sum_{i=1}^{N} \mathbb{P}_{i}\left(x_{\Omega^{\prime}}, \Omega\right)$ is the arithmetic mean of all $\mathbb{P}_{i}\left(x_{\Omega^{\prime}}, \Omega\right)$.

To obtain the bound for $\mathbb{E}\left[\forall_{i \in U} I_{i}=1 \mid \sum_{i=1}^{N} I_{i} \geq r\left(\Omega, \partial \Omega^{\prime}\right) N\right]$, recall that $1-\sigma$ is the probability that an element of $U$ is such that $I_{i}=0$. We also note that if at least $r\left(\Omega, \partial \Omega^{\prime}\right) N$ of the elements of $U$ are ones, then there are $N-r\left(\Omega, \partial \Omega^{\prime}\right) N$ zeros, that is, $N-r\left(\Omega, \partial \Omega^{\prime}\right) N$ players are not in set $U$. It follows that

$$
\mathbb{E}\left[\forall_{i \in U} I_{i}=1 \mid \sum_{i=1}^{N} I_{i} \geq r\left(\Omega, \partial \Omega^{\prime}\right) N\right]=(1-\sigma)^{\left(1-r\left(\Omega, \partial \Omega^{\prime}\right)\right) N}
$$

Equations (2.9.6) together with (2.9.7) when substituted into (2.9.2) yield,

$$
K_{P}\left(\frac{\left(\sigma \mathbb{P}_{A}\left(x_{\Omega^{\prime}}, \Omega\right)+1-\sigma\right)}{(1-\sigma)^{\left(1-r\left(\Omega, \partial \Omega^{\prime}\right)\right)}}\right)^{N} \leq \mathbb{P}\left(\sum_{i=1}^{N} I_{i} \geq r\left(\Omega, \partial \Omega^{\prime}\right) N\right) \leq\left(\frac{\left(\sigma \mathbb{P}_{A}\left(x_{\Omega^{\prime}}, \Omega\right)+1-\sigma\right)}{(1-\sigma)^{\left(1-r\left(\Omega, \partial \Omega^{\prime}\right)\right)}}\right)^{N},
$$


where $K_{P}=\frac{K_{A}}{K_{1}}$. If we choose $\sigma$ that optimizes the quantity $\varphi=\frac{\left(\sigma \mathbb{P}_{A}\left(x_{\Omega^{\prime}}, \Omega\right)+1-\sigma\right)}{(1-\sigma)^{\left(1-r\left(\Omega, \partial \Omega^{\prime}\right)\right)}}$ (by equating the derivative to zero and solving for $\sigma$ ) and substituting back gives

$$
\begin{aligned}
& K_{P}\left(\left(\frac{\mathbb{P}_{A}\left(x_{\Omega^{\prime}}, \Omega\right)}{r\left(\Omega, \partial \Omega^{\prime}\right)}\right)^{r\left(\Omega, \partial \Omega^{\prime}\right)}\left(\frac{1-\mathbb{P}_{A}\left(x_{\Omega^{\prime}}, \Omega\right)}{1-r\left(\Omega, \partial \Omega^{\prime}\right)}\right)^{1-r\left(\Omega, \partial \Omega^{\prime}\right)}\right)^{N} \leq \mathbb{P}\left(\sum_{i=1}^{N} I_{i} \geq r\left(\Omega, \partial \Omega^{\prime}\right) N\right) \\
& \leq\left(\left(\frac{\mathbb{P}_{A}\left(x_{\Omega^{\prime}}, \Omega\right)}{r\left(\Omega, \partial \Omega^{\prime}\right)}\right)^{r\left(\Omega, \partial \Omega^{\prime}\right)}\left(\frac{1-\mathbb{P}_{A}\left(x_{\Omega^{\prime}}, \Omega\right)}{1-r\left(\Omega, \partial \Omega^{\prime}\right)}\right)^{1-r\left(\Omega, \partial \Omega^{\prime}\right)}\right)^{N}
\end{aligned}
$$

Re-expressing (2.9.9) in exponential form results to

$$
\begin{aligned}
& K_{P} e^{N\left[r\left(\Omega, \partial \Omega^{\prime}\right) \ln \left(\frac{\mathbb{P}_{A}\left(x_{\Omega^{\prime}}, \Omega\right)}{r\left(\Omega, \partial \Omega^{\prime}\right)}\right)+\left(1-r\left(\Omega, \partial \Omega^{\prime}\right)\right) \ln \left(\frac{1-\mathbb{P}_{A}\left(x_{\Omega^{\prime}}, \Omega\right)}{1-r\left(\Omega, \partial \Omega^{\prime}\right)}\right)\right]} \leq \mathbb{P}\left(N_{t+1}\left(x_{\Omega^{\prime}}\right) \geq R\left(\Omega, \partial \Omega^{\prime}\right) \mid \mathbf{x}_{t} \in \Omega\right) \\
& \leq e^{N\left[r\left(\Omega, \partial \Omega^{\prime}\right) \ln \left(\frac{\mathbb{P}_{A}\left(x_{\Omega^{\prime}}, \Omega\right)}{r\left(\Omega, \partial \Omega^{\prime}\right)}\right)+\left(1-r\left(\Omega, \partial \Omega^{\prime}\right)\right) \ln \left(\frac{1-\mathbb{P}_{A}\left(x_{\Omega^{\prime}}, \Omega\right)}{1-r\left(\Omega, \partial \Omega^{\prime}\right)}\right)\right]}
\end{aligned}
$$

Note that each $\mathbb{P}_{i}\left(x_{\Omega^{\prime}}, \Omega\right) \ll 1$, hence $\ln \left(1-\mathbb{P}_{A}\left(x_{\Omega^{\prime}}, \Omega\right)\right) \ll \ln \left(1-r\left(\Omega, \partial \Omega^{\prime}\right)\right)$ such that

$$
\begin{aligned}
K_{P} e^{-N\left[F\left(r\left(\Omega, \partial \Omega^{\prime}\right)\right)-r\left(\Omega, \partial \Omega^{\prime}\right) \ln \left(\mathbb{P}_{A}\left(x_{\Omega^{\prime}}, \Omega\right)\right)\right]} & \leq \mathbb{P}\left(N_{t+1}\left(x_{\Omega^{\prime}}\right) \geq R\left(\Omega, \partial \Omega^{\prime}\right) \mid \mathbf{x}_{t} \in \Omega\right) \\
& \leq e^{-N\left[F\left(r\left(\Omega, \partial \Omega^{\prime}\right)\right)-r\left(\Omega, \partial \Omega^{\prime}\right) \ln \left(\mathbb{P}_{A}\left(x_{\Omega^{\prime}}, \Omega\right)\right)\right]}
\end{aligned}
$$

where $F\left(r\left(\Omega, \partial \Omega^{\prime}\right)\right)=r\left(\Omega, \partial \Omega^{\prime}\right) \ln \left(\frac{r\left(\Omega, \partial \Omega^{\prime}\right)}{1-r\left(\Omega, \partial \Omega^{\prime}\right)}\right)+\ln \left(r\left(\Omega, \partial \Omega^{\prime}\right)\right)$. Recall that the cost of the transition $\Omega \rightarrow \partial \Omega^{\prime}$ is

$$
c\left(\Omega, \partial \Omega^{\prime}\right)=-r\left(\Omega, \partial \Omega^{\prime}\right) \ln \left(\mathbb{P}_{A}\left(x_{\Omega^{\prime}}, \Omega\right)\right) .
$$

Hence,

$$
K_{P} e^{-N\left[c\left(\Omega, \partial \Omega^{\prime}\right)+F\left(r\left(\Omega, \partial \Omega^{\prime}\right)\right)\right]} \leq P_{\mathcal{E}}\left(\Omega, \partial \Omega^{\prime}\right) \leq e^{-N\left[c\left(\Omega, \partial \Omega^{\prime}\right)+F\left(r\left(\Omega, \partial \Omega^{\prime}\right)\right)\right]}
$$

\subsubsection{Proof of Theorem 1}

(i). From the Lemma 5 and Proposition 4 it directly follows that

$$
\frac{\pi_{\varepsilon}\left(\tilde{\Omega}^{\prime}\right)}{\pi_{\varepsilon}(\tilde{\Omega})} \leq \frac{\max _{\Omega^{\prime \prime}} P_{\mathcal{\varepsilon}}\left(\Omega, \partial \Omega^{\prime \prime}\right)}{P_{\varepsilon}\left(\Omega^{\prime}, \partial \Omega\right)} \leq K \exp \left\{N\left(Q\left(\Omega^{\prime}, \Omega\right)-Q_{\min }(\Omega)\right)\right\}
$$

Implying that the subset of states $\bar{\Omega}$ containing epsilon stable state is the for which $\left(Q\left(\Omega^{\prime}, \bar{\Omega}\right)<Q_{\min }(\bar{\Omega})\right)$ for all $\Omega^{\prime} \neq \bar{\Omega}$. That is, $Q(\bar{\Omega})<0$.

(ii). For every closed communication class say $\Omega_{J}$, let $H_{\Omega_{j} \Omega_{J}}$ be the typical directed path from any other closed communication class $\Omega_{j}$ to $\Omega_{J}$. Then from 13 


$$
\begin{aligned}
\frac{\pi_{\varepsilon}\left(\tilde{\Omega}^{1}\right)}{\pi_{\varepsilon}\left(\tilde{\Omega}^{J}\right)} & =\frac{\pi_{\varepsilon}\left(\tilde{\Omega}^{1}\right)}{\pi_{\varepsilon}\left(\tilde{\Omega}^{2}\right)} \cdots \frac{\pi_{\varepsilon}\left(\tilde{\Omega}^{J-1}\right)}{\pi_{\varepsilon}\left(\tilde{\Omega}^{J}\right)} \leq \frac{P_{\varepsilon}\left(\Omega^{2}, \Omega^{2^{c}}\right)}{P_{\mathcal{\varepsilon}}\left(\Omega^{1}, \Omega^{2}\right)} \cdots \frac{P_{\varepsilon}\left(\Omega^{J} \Omega^{J^{c}}\right)}{P_{\mathcal{\varepsilon}}\left(\Omega^{J-1}, \Omega^{J}\right)} \\
& \leq K_{c} \exp \left\{N\left(\sum_{j=1}^{J-1}\left(Q\left(\Omega_{j}, \Omega_{j+1}\right)-Q_{\min }\left(\Omega_{j+1}\right)\right)\right)\right\}
\end{aligned}
$$

Where $\xi\left(H_{\Omega_{1} \Omega_{J}}\right)=\sum_{j=1}^{J-1}\left(Q\left(\Omega_{j}, \Omega_{j+1}\right)-Q_{\min }\left(\Omega_{j+1}\right)\right)$. Now, consider the maximum probability paths (or equivalently the minimum cost paths) from any closed communication class $\Omega_{j}$ to another $\Omega_{J}$. That is $\arg \max _{H_{\Omega_{j} \Omega_{J}} \in \mathscr{H}_{\Omega_{j} \Omega_{J}}} \xi\left(H_{\Omega_{j} \Omega_{J}}\right)$.

Then the epsilon stable set $\bar{\Omega}$, for which $\pi_{\mathcal{\varepsilon}}(\bar{\Omega})>\pi_{\mathcal{\varepsilon}}(\Omega) \forall \Omega \neq \bar{\Omega}$, is that in which $\xi\left(H_{\Omega \bar{\Omega}}\right)<0$. Or equivalently $\xi(\bar{\Omega})=\max _{\Omega \neq \bar{\Omega}} \min _{H_{\Omega \bar{\Omega}} \in \mathscr{H}_{\Omega \bar{\Omega}}} \xi\left(H_{\Omega \bar{\Omega}}\right)<0$.

(iii). From Lemma 2, the bounds for $P_{\mathcal{E}}(g)=\prod_{\left(\Omega, \Omega^{\prime}\right) \in g} P_{\mathcal{E}}\left(\Omega, \Omega^{\prime}\right)$ are

$$
K_{1} e^{-N Q(g)} \leq P_{\varepsilon}(g) \leq e^{-N Q(g)}
$$

where $Q(g)=\sum_{\left(\Omega, \Omega^{\prime}\right) \in g} Q\left(\Omega, \partial \Omega^{\prime}\right)$.

$\pi_{\varepsilon}(\Omega) \leq K_{e} \frac{\max _{g \in G(\{\Omega\})} P_{\varepsilon}(g)}{\min _{\Omega^{\prime} \in \Omega} \min _{g \in G\left(\left\{\Omega^{\prime}\right\}\right)} P_{\varepsilon}(g)}=K_{e} \exp \left\{N\left(\min _{\Omega^{\prime} \in \Omega^{\prime}} \min _{g \in G\left(\left\{\Omega^{\prime}\right\}\right)} Q(g)-\min _{g \in G(\{\Omega\})} Q(g)\right)\right\}$

where $K_{e}$ is some constant;

$$
K_{e}=\frac{\# G(\{\Omega\})}{\# \Omega \# G\left(\left\{\Omega^{\prime}\right\}\right)}
$$

The epsilon stable set $\bar{\Omega}$, which maximizes the stationary distribution, is then that for which $\min _{g \in G(\{\bar{\Omega}\})} Q(g)=\min _{\Omega^{\prime} \in \mathbf{\Omega}^{\prime}} \min _{g \in G\left(\left\{\Omega^{\prime}\right\}\right)} Q(g)$. Or equivalently,

$$
\mathscr{S}(\bar{\Omega})=\min _{\Omega \in \Omega} \mathscr{S}(\Omega)=\min _{\Omega \in \Omega} \min _{g \in G(\{\Omega\})} \sum_{\left(\Omega_{j}, \Omega_{\kappa}\right) \in g} Q\left(\Omega_{j}, \Omega_{\kappa}\right)
$$

\subsubsection{Proof of Proposition 2}

(i). The proof involves comparing the measures $Q\left(\Omega, \Omega^{\prime}\right)$ and $V\left(\Omega, \Omega^{\prime}\right)$ for epsilon stability and stochastic stability respectively. When $\mathcal{P}_{i}(x \mid \mathbf{x})=\frac{1}{m}$ for all $i$, the for each pair $\left(\Omega, \Omega^{\prime}\right)$,

$$
\begin{gathered}
\ln \mathbb{P}_{A}=\ln \left(\frac{1}{N} \sum_{i=1}^{N} \varepsilon_{i}\right)+\ln m^{-1} \\
Q\left(\Omega, \Omega^{\prime}\right)=-r\left(\Omega, \partial \Omega^{\prime}\right)\left(\ln \left(\frac{1}{N} \sum_{i=1}^{N} \varepsilon_{i}\right)+\ln m^{-1}\right)+F\left(r\left(\Omega, \partial \Omega^{\prime}\right)\right)
\end{gathered}
$$


Since $\ln m^{-1}$ is identical for every pair of closed communication classes, $-0.7 \leq$ $F\left(r\left(\Omega, \partial \Omega^{\prime}\right)\right) \leq 0$ and that each $\varepsilon_{i}$ is state independent, then the only relevant quantities in the computation of epsilon stable sets are $r\left(\Omega, \partial \Omega^{\prime}\right)$. Let $\varepsilon_{A}=\frac{1}{N} \sum_{i=1}^{N} \varepsilon_{i}$, the for each $V\left(\Omega, \Omega^{\prime}\right)$ we have,

$$
V\left(\Omega, \Omega^{\prime}\right)=\lim _{\varepsilon_{A} \rightarrow 0}-\varepsilon_{A} r\left(\Omega, \partial \Omega^{\prime}\right)\left(\ln \left(\frac{1}{N} \sum_{i=1}^{N} \varepsilon_{i}\right)+\ln m^{-1}\right)=r\left(\Omega, \partial \Omega^{\prime}\right)
$$

Implying that the only relevant quantities in the computations of stochastically stable sets are $r\left(\Omega, \partial \Omega^{\prime}\right)$. Hence epsilon and stochastically stable sets must be identical.

(ii). For $\mathbb{P}_{i}(x, \mathbf{x}) \neq \frac{1}{m}$ we have,

$$
Q\left(\Omega, \Omega^{\prime}\right)=-r\left(\Omega, \partial \Omega^{\prime}\right)\left(\ln \left(\frac{1}{N} \sum_{i=1}^{N} \varepsilon_{i}\right)+\ln \left(\frac{1}{N} \sum_{i=1}^{N} \mathcal{P}_{i}\left(x_{\Omega^{\prime}}, \Omega\right)\right)\right)+F\left(r\left(\Omega, \partial \Omega^{\prime}\right)\right)
$$

If the mistakes probability mass functions are bounded, that is $0<\mathcal{P}_{i}\left(x_{\Omega^{\prime}}, \Omega\right)<1$ $\forall i \in \mathcal{N}$ and for all $\Omega$, then so must be the averages $\frac{1}{n} \sum_{i=1}^{n} \mathcal{P}_{i}\left(x_{\Omega^{\prime}}, \Omega\right)$. Implying that there must exist an $\varepsilon^{\prime}$ sufficiently small such that for all $\varepsilon_{A}<\varepsilon^{\prime}$, $\varepsilon_{A}<$ $\ln \left(\frac{1}{N} \sum_{i=1}^{N} \mathcal{P}_{i}\left(x_{\Omega^{\prime}}, \Omega\right)\right)$. Whenever $\varepsilon_{A}<\varepsilon^{\prime}$ the relevant quantities in the computation of epsilon stable sets are $r\left(\Omega, \partial \Omega^{\prime}\right)$, In which case epsilon stable sets will be identical to stochastically stable sets. 


\section{STOCHASTIC EVOLUTION IN NETWORKS}

Abstract: This Chapter develops methods for stochastic evolutionary equilibrium selection in networks. We derive conditions for asymptotic global convergence to be global or path-wise contagion, and that the network must be strongly connected. Strategies that are either globally or path-wise contagious are then those that are asymptotically globally stable. Asymptotic global stability is robust to the model of mistakes and addition of strictly dominated strategies. We show that for a given payoff structure and hence relative payoff gains, a network can be designed to make at least one strategy asymptotically globally stable. Similarly, for a given network structure and set of strategies, one can determine the relative payoff gains that lead to a given strategy to be globally stable. We briefly discuss the practical and empirical relevance of these findings.

\subsection{INTRODUCTION}

As partially discussed in Chapter 2 above, in analyzing long-run outcomes of stochastic evolutionary processes, Foster and Young [41] and Kandori et al. [57] use the concept of stochastic stability. Stochastically stable outcomes are those that occur with positive probability at the limit of randomness. Fairly concrete results have been established with regard to stochastic stability in $2 \times 2$ coordination games. For example Young [113] and Kandori et al. [57], Blume [19] show that in $2 \times 2$ coordination games and under global interactions, risk-dominant strategies are stochastically stable. ${ }^{1}$ Maruta [72] derives equivalent results for $m \times m$ coordination games, showing that globally risk-dominant strategies are selected in the long-run. ${ }^{2}$ Michihiro and Rob

1 In a 2 x2 symmetric game with two symmetric equilibria in pure strategies, one risk-dominates another if and only if the equilibrium strategy is a unique best response to any mixture that gives it at least a probability of one half.

2 In an $m \times m$ symmetric game, a strategy is globally risk-dominant if it is a unique best response to any mixture that gives it at least a probability of one half. 
[74] show that globally pairwise risk-dominant strategies are stable in the long-run only under strict conditions they refer to as total bandwagon property and marginal bandwagon property. 3 Under local interactions and $2 \times 2$ games, [38], Lee and Valentinyi [67] and Lee et al. [66] show risk-dominant strategies are stable in the long-run.

In spite of these strong predictions, questions regarding robustness of and convergence rates to long-run stable outcomes do exist. For example Bergin and Lipman [15] show that for given $m \times m$ asymmetric game, the outcomes that are stochastically stable strictly depend on the assumptions made concerning the structure of experimentation by players. [59] show that for a given model of mistakes, any outcome can be made stochastically stable by appropriately adding strictly dominated strategies. Alós-Ferrer and Weidenholzer [8] demonstrate that contrary to the predictions made in Michihiro and Rob [74] regarding globally pairwise risk dominant strategies under global interactions, under local interactions and beyond $4 \times 4$ coordination games, globally pairwise risk dominant strategies need not be selected. Ellison [37] showed that under global interactions, the expected waiting times to stochastically stable outcomes are long.

This Chapter studies evolutionary processes in networks and $m \times m$ symmetric games. We follow the customary behavioral and dynamics assumptions of Darwinian dynamics and with focus on establishing conditions for play to converge in the long-run and on how to identify stable strategies. More specifically, agents are assumed to base their decisions on the distribution of strategies in the previous period and not the entire history of play. Unlike in the case of global interactions, local interactions imply that information available to each agent is limited and is simply the distribution of strategies in their neighborhood. We establish conditions for asymptotic global convergence and stability, that is the long-run outcomes at the limit of population size rather than limit of noise. In so doing, we preserve the necessity of maintaining positive noise levels in evolutionary models. That is positive noise levels captures the general assumption of bounded rationality and experimentation embedded in the models.

These two fairly standard assumptions, limited information (local interactions) and positive noise levels, circumvent some of the above mentioned limitations of evolutionary processes in the following ways. First, several authors have shown that under local interactions the expected waiting times to the long-run stable state from any other state are bounded (e.g. Ellison [37], Young [116], Montanari and Saberi [77]).

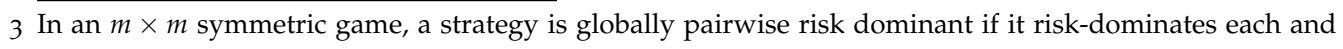
every other strategy. Global pairwise risk dominance is a weaker notion compared to that of global risk dominance according to Maruta [72]. 
The limitation concerning the convergence rates is thus not necessarily critical under local interactions, specially when the noise levels are kept positive.

Secondly, under local interactions asymptotic global convergence occurs for strongly connected networks and additional conditions we describe below. We show that for a given structure of interactions, strategies that are globally and/or path-wise contagious are globally stable for a sufficiently large population size. By contagious we mean a strategy spreads by best-response once a small fraction of players have adopted it. A strategy is globally contagious if it is uniquely contagious after pair-wise comparison with all other strategies. A strategy, say $a$, is path-wise contagious relative to another say $b$ if there exists a directed path from $b$ to $a$, such that each strategy on the path is contagious. For any given game however, even when no strategy is globally contagious it is possible to construct a range of network families for which at least one strategy is path-wise contagious. The relationship between global and path-wise contagion on one hand with interaction and the underlying game structures on the other hand is not linear. For a given game, sparsely connected networks tend to favor global and path-wise contagion. For a given network structure, strategies with the largest relative payoff gains are those that are potentially globally and path-wise contagious. 4

The notion of contagion in networks under strategic interactions as defined here is related to that in Morris [78]. Any given arbitrary network has a critical contagion threshold such that contagion occurs if and only if the relative payoff gain is below the contagion threshold. In this regard, the analysis in this Chapter is closely related to Morris [78] in making use of the notion of contagion to characterize long-run outcomes of evolutionary processes in networks. The main difference resides in the fact that we focus on stochastic rather than deterministic dynamics. In so doing, we are able to make unique predictions of long-run stable outcomes for $m \times m$ coordination games. Similarly, we focus on characterizing long-run stable outcomes as opposed to determining the properties of networks that influence contagion.

Global contagion as we define here is related to the concept of $p$-dominance according to Morris et al. [79]. Generally, an action pair in a two-player game is said to be $p$-dominant if each action is a best response to any belief that the other player takes the action in this pair with probability at least $p$. For the local interaction game we consider, a strategy is $p$-dominant if for all players, it is the unique best-response when it is played by at least proportion $p$ of the neighbors. This implies that for a network with contagion threshold $p$, a strategy that is $p$-dominant is also globally

4 For any given matrix game, the relative payoff gain of strategy say $a$ in relation to another say $b$, is equivalent to the fraction of opponents that must play $a$ for that agent to switch from playing $b$ to playing $a$. 
contagious. Path-wise contagion in the context of local interaction game as defined in this Chapter is however a novel notion, and is less restrictive compared to global contagion.

In relation to the analysis in Alós-Ferrer and Weidenholzer [8], global and pathwise contagion are alternative methods that focus on the properties of the underlying network and game. Alós-Ferrer and Weidenholzer [8] focus on establishing conditions for selection of $\frac{1}{2}$-dominant strategies, which occurs in networks with contagion threshold of $\frac{1}{2}$; for example the cyclic interaction structure. Here, we do not place restrictions on the contagion threshold and hence network topology. Most importantly however, global and path-wise contagion as opposed to the notion of Global pairwise risk-dominance and the partial bandwagon properties in Alós-Ferrer and Weidenholzer [8] can be employed to make prediction for long-run outcomes in $m \times m$ and not just $3 \times 3$ coordination games.

The closely related literature with regard to $p$-dominance is the recent papers by Sandholm [99] and Sandholm et al. [100]. They show that the deterministic dynamics where players sample strategies of $k$-randomly chosen other players in the population leads to almost global convergence to $\frac{1}{k}$-dominant strategies. In relation to the definitions in this Chapter, $\frac{1}{k}$-dominant strategies would be those that are globally contagious whenever the maximum number of neighbors any player has is $k$. The contrast with what we do in this Chapter is that we study non-deterministic processes under general interaction structures, hence derive results for general interaction topologies and revision probabilities. Secondly, we emphasize evolutionary processes with positive noise levels. This enables us to derive results pertaining global convergence rather than almost global convergence.

Thirdly, we show that asymptotic globally stable outcomes are robust to addition of dominated strategies, circumventing the limitation of stochastic stability pointed out by [59]. The reason for the robustness of asymptotic global stability to both addition of strictly dominated strategies and model of mistakes is that the factors that determine global stability under local interactions (global and path-wise contagion) are robust to both phenomena.

The most relevant aspect of our results is that for a given payoff structure and hence relative payoff gains, it is possible to design a network such that at least one strategy is asymptotically globally stable. Similarly, for a given network structure and set of strategies, one can determine the relative payoff gains that lead to a given strategy to be globally stable. Moreover, these findings are robust to the model of mistakes, circumventing the limitations pointed out by Bergin and Lipman [15]. 
The findings in this Chapter have both practical and empirical implications. First, since the model of mistakes does not play a role in determining the long-run stable outcomes (but perhaps only the rates of convergence), then for any given game (e.g. institutional or technological adoption) if the network and payoff structures are known a priori, the level of noise/experimentation that is characteristic to the given interaction environment can be estimated. This can be done using historical data for competing technological products for example. For example Young [114] fits adoption curves to data on diffusion of Hybrid Corn (based on the empirical work of Ryan and Gross [97]) to distinguish between three diffusion processes; contagion, social influence and social learning. A similar exercise can be performed for a specific learning process then estimate the desirable level of experimentation. This exercise is feasible since the network of interactions can be empirically determined. Recently, there has been a growing literature both theoretical and empirical in the field of social networks. Most of this literature is devoted to characterizing the distributional structure of real world social networks (see for example Newman et al. [86] and Newman [87]). Determining the level of experimentation that is characteristic to a given institutional structure or technology field is particularly relevant if we view the process of experimentation as an abstraction from the actual process through which new technologies and institutions emerge. The second practical relevance of our result is that, by relating network structure to relative payoff gains it is possible to determine for a given network of interaction the required level of incremental innovation for one product to take over the market. That is, for any given network one can determine the level of relative payoff gain for a given strategy to be globally contagious independently of the level of noise and model of mistakes. Similarly, if we think of competing scientific theories as exhibiting strategic complementarity, then for a given network of interactions between scientists (which can be constructed from co-authorship or cross-citations) one can determine the level of empirical evidence/support (hence relative payoff gains) that can lead to one theory to be firmly accepted.

The remainder of the Chapter is organized as follows. In section 3.2 we introduce the general framework of stochastic evolutionary dynamics in networks. Section $3 \cdot 3$ defines the solution concept and provides results for global convergence and stability. Sections 3.4 and 3.5 developed the concepts of global and path-wise contagion, explicitly characterizing how the network and payoff structures interactively shape long-run stability. 


\subsection{THE MODEL}

We consider an $m$ strategy matrix game $\Gamma\left(X,\left\{U_{i}\right\}_{i \in \mathcal{N}}\right)$ played by a set $\mathcal{N}=$ $\{1, \cdots, i, \cdots\}$ of players. Where $X=\{a, b, c, \cdots\}$ is the strategy set identical for all players and $U \in \mathbb{R}^{m \times m}$ is the associated payoff matrix such that $U_{i}(a, b)$ is the base payoff to $i$ for playing $a$ when the opponent plays $b$. Whenever the a finite population is referred $N$ will denote the population size. Players revise their strategies simultaneously at discrete time intervals $t=1,2, \cdots$. At each $t$, each player evaluates the available strategies and chooses that which maximizes the expected payoffs. This evaluation process in based on strategies of opponents in the neighborhood. The neighborhoods form each player's social network. Generally, the network of interactions can be modeled in a graph theoretical manner.

Let $G(N, E)$ be a graph with $N$ vertices representing the number of agents and $E$ edges linking different pairs of agents, such that a graph $g_{i j}$ defines the connection between $i$ and $j$. If $g_{i j}=1$ then a directed link exists from $i$ to $j$, and zero implies otherwise. $G(N, E)$ is thus a directed network describing the relationship of any one agent with every other agent in the population. The adjacency matrix $\mathscr{A}$ of $G(N, E)$ is defined as an $N \times N$ matrix with entries being the elements of $g_{i j}$. The neighborhood of agent $i, \mathcal{N}_{i}$, is defined as $\mathcal{N}_{i}=\left\{j \in N \mid g_{i j}=1\right\}$, and gives the set of players to which $i$ is linked to. The cardinality $\# \mathcal{N}_{i}=k_{i}$, is the degree of $i$.

We assume that each player plays the same strategy against all the neighbors. That is given state $\mathbf{x}$, where $\mathbf{x}_{-i}$ denotes the population state with $i$ excluded, the expected payoff to $i$ for playing strategy $a$ is

$$
U_{i}\left(a, \mathbf{x}_{-i}\right)=\sum_{j \in \mathcal{N}_{i}} J_{i j} U\left(a, x^{j}\right),
$$

where $x^{j}$ is the $j^{\text {th }}$ coordinate of $\mathbf{x}$. The parameter $J_{i j}$ takes on values in the closed interval $[0,1]$. It is the weight that $i$ attaches to the interaction with $j$, such that if $i$ weights all the neighbors equally then $J_{i j}=\frac{1}{k_{i}}$ for all $j \in \mathcal{N}_{i}$. The model of stochastic evolution in networks is then defined as follows.

Definition 4: A model of stochastic evolution in networks is a quadruple $\left(\mathbf{X}, P_{N}, P_{N, \varepsilon}, G_{N}\right)$ consisting of

1) A set $\mathbf{X}$ referred to as the state space of the model.

2) A family of interaction networks $G_{N}$ indexed by $N$ as the number of vertices and $E_{N}$ as corresponding number of edges such that

$$
\lim _{N \rightarrow \infty} \frac{\left|E_{N}\right|}{N(N-1)}<1
$$


3) A family of Markov transition matrices $P_{N}$ on $\mathbf{X}$ indexed by $N \geq 3$.

4) A family of Markov transition matrices $P_{N, \varepsilon}$ on $\mathbf{X}$ indexed by $N \geq 3$ and the set $\varepsilon=\left(\varepsilon_{1}, \cdots, \varepsilon_{N}\right)$, such that

(i) $P_{N, \varepsilon}$ is ergodic for all $\varepsilon_{i}>0$.

(ii) $P_{N, \varepsilon}$ is continuous in $\varepsilon$ and $P_{N, 0}=P_{N}$.

(iii) Each $\varepsilon_{i}$ can be state dependent but are independent of $N$.

Condition (3.2.2) of the model places restriction on the structure of the network as the population size grows. It states that the density of the network as $N$ grows should be bounded away from one. A complete network (in which each player interacts with every other player) has density of one; (3.2.2) therefore rules out complete networks and all network families for which the number (or mass) of edges grows proportionally with its volume: $N(N-1)$. As already established in Ellison [37], for complete networks the radii of all basins of attraction are increasing functions of $N$. Condition (3.2.2) rules out these cases and is necessary for the validity of results in Theorem 2 (ii) and (iii) below.

The Markov transition matrix $P_{N}$ is based on the unperturbed dynamics and $P_{N}(\mathbf{x}, \mathbf{y})$ is the probability that state $\mathbf{x}$ is followed by $\mathbf{y}$. Since we assume best response dynamics rather than say imitation, $P_{N}(\mathbf{x}, \mathbf{y})$ is the probability that state $\mathbf{y}$ is reached when each player switches to a strategy $y^{i}$ that is best-response to $\mathbf{x}$. That is for each $i$

$$
B R_{i}\left(y^{i}, \mathbf{x}\right)= \begin{cases}1 & \text { if } y^{i} \in \arg \max _{b \in X} U_{i}\left(b, \mathbf{x}_{-i}\right) \\ 0 & \text { otherwise. }\end{cases}
$$

The fourth element of the model specifies the nature of random perturbations. There are two main ways to capture the aspect of randomness. The mistakes resulting from experimentation (on strategies) and randomness resulting from expected payoffs perturbations. Under the former, the probability that player $i$ chooses action $a$ given $\mathbf{x}$ is

$$
\mathbb{P}_{i}(a, \mathbf{x})=\left(1-\varepsilon_{i}(\mathbf{x})\right) B R_{i}(a, \mathbf{x})+\varepsilon_{i}(\mathbf{x}) \mathcal{P}_{i}(a, \mathbf{x})
$$

where $\sum_{a \in X} \mathcal{P}_{i}(a, \mathbf{x})=1$ for each $i \in \mathcal{N}$ and $a \in \mathbf{X}$.

Condition (iii) of element 4 of the model implies that each $\varepsilon_{i}(\mathbf{x})$ and $\mathcal{P}_{i}(a, \mathbf{x})$ can be state dependent but are independent of $N$. This condition is necessary to rule out cases in which the likelihood of mistakes is a decreasing function of $N$. A distinction should however be made between the likelihood of mistakes and the number of mistakes. While the likelihood of making a mistake to transition from one state to another is independent of $N$, the number of mistakes required to exit a subset of 
states can grow with $N$. The special case of dynamics (3.2.4) are Kandori et al. [57] and Young [113] where mutation rates are state and player independent.

Under the case of expected payoff perturbations, $i^{\prime}$ s objective is to choose a strategy $a$ that maximizes the perturbed payoff $U_{i}\left(a, \mathbf{x}_{-i}\right)+\varepsilon_{a}$, that is

$$
a \in \underset{b \in X}{\arg \max } U_{i}\left(b, \mathbf{x}_{-i}\right)+\varepsilon_{b}
$$

where $\varepsilon_{b}$ 's are the random components of the expected payoff. The special cases of expected payoff perturbations are those in which the components of $\varepsilon=\left(\varepsilon_{a}, \varepsilon_{b}, \cdots\right)$ are assumed to be independently distributed. For example the logit and multinomial probit in Blume [20] and Myatt and Wallace [82] respectively.

\subsection{ASYMPTOTIC GLOBAL CONVERGENCE}

Given the conditions for random perturbations above, the process $P_{N, \varepsilon}$ has a well defined unique invariant distribution $\pi_{N, \varepsilon}=\lim _{t \rightarrow \infty} \mathbf{q}_{0} P_{N, \varepsilon^{\prime}}^{t}$, where $\mathbf{q}_{t}$ is the vector of probability mass functions at period $t$. It describes the amount of time the process spends in each state in the long-run or equivalently the long-run probability of each state. We denote by $\Omega$ for a typical closed communication class or equivalently limit sets of the equivalent process without mistake, that derives from the dynamics in (3.2.3). The set of all such sets is denoted by $\Omega$. In pure coordination games for example, the closed communication classes include the singleton sets in which all players play the same strategy. Generally however, closed communication classes can include sets that are cycles and those in which players use different strategies. When interactions are local, the number of closed communication classes may increase depending on the network topology.

Let $\partial \Omega$ denote the boundary of a closed communication class $\Omega$. That is, all states in the basin of attraction of $\Omega$ with $\Omega$ excluded. 5 The basin of attraction of $\Omega$ is then $\tilde{\Omega}=\partial \Omega \cup \Omega$. We write $\left(\tilde{\Omega}, \tilde{P}_{N, \varepsilon}\right)$ for the chain with transitions between elements $\tilde{\Omega}$. Asymptotic global convergence is then formally defined as follows

Definition 5: Given a family of networks $G_{N}$ indexed by $N$, a subset of states $\Omega$ is said to be asymptotically globally stable if $\lim _{N \rightarrow \infty} \pi_{N, \varepsilon}(\Omega)>0$

That is, asymptotically globally stable sets are those where the probability with which it is played in the long-run is positive at the limit of the population size. The analysis of chain $P_{N, \varepsilon}$ thus involves the characterization of the stationary distribution. Here, we make use of the quotients method. More specifically, the following Lemma.

5 The basin of attraction of a closed communication class $\Omega$ is defined as $\tilde{\Omega}=\left\{\mathbf{x} \in \mathbf{X} \mid \mathbb{P}\left(\exists T\right.\right.$ s.t $\mathbf{x}_{t} \in \Omega \forall t>$ $\left.\left.T \mid \mathbf{x}_{0}=\mathbf{x}\right)=1\right\}$. That is, the set of states from which the chain without noise converges to $\Omega$. 
Lemma 5: Let $\Omega$ and $\Omega^{\prime}$ be any two closed communication classes such that $P_{N, \varepsilon}\left(\Omega^{\prime}, \Omega\right)$ is the probability of the transition from some state in $\Omega^{\prime}$ to another in $\Omega$. Let also $\Omega^{c}$ be the complement of $\Omega$. Then

$$
\frac{\pi_{N, \varepsilon}\left(\tilde{\Omega}^{\prime}\right)}{\pi_{N, \varepsilon}(\tilde{\Omega})} \leq \frac{P_{N, \varepsilon}\left(\Omega, \Omega^{c}\right)}{P_{N, \varepsilon}\left(\Omega^{\prime}, \Omega\right)}
$$

Similarly, if $\Omega_{1} \rightarrow \Omega_{2} \rightarrow \cdots \rightarrow \Omega_{j} \rightarrow \cdots \rightarrow \Omega_{J}$ is a directed path from $\Omega_{1}$ to $\Omega_{J}$, then

$$
\frac{\pi_{N, \varepsilon}\left(\tilde{\Omega}_{1}\right)}{\pi_{N, \varepsilon}\left(\tilde{\Omega}_{J}\right)} \leq \frac{P_{N, \varepsilon}\left(\Omega_{2}, \Omega_{2}^{c}\right)}{P_{N, \varepsilon}\left(\Omega_{1}, \Omega_{2}\right)} \cdots \frac{P_{N, \varepsilon}\left(\Omega_{J}, \Omega_{J}^{c}\right)}{P_{N, \varepsilon}\left(\Omega_{J-1}, \Omega_{J}\right)}
$$

Proof. See Appendix 3.8.1

The probabilities $P_{N, \varepsilon}\left(\Omega, \Omega^{\prime}\right)$ are in principle single step probabilities involving states belonging to two different closed communication classes. Under Lemma 5, we say a (subset of) closed communication class is asymptotically globally stable if its basin of attraction is asymptotically globally stable. The first step of the analysis therefore involves deriving bounds for the probabilities $P_{N, \varepsilon}\left(\Omega, \Omega^{\prime}\right)$. It is convenient to split the transition $\Omega \rightarrow \Omega^{\prime}$ into two transitions, from $\Omega \rightarrow \partial \Omega^{\prime}$ and $\partial \Omega^{\prime} \rightarrow \Omega^{\prime}$.

$$
P_{N, \varepsilon}\left(\Omega, \Omega^{\prime}\right)=P_{N, \varepsilon}\left(\Omega, \partial \Omega^{\prime}\right) P_{N, \varepsilon}\left(\partial \Omega^{\prime}, \Omega^{\prime}\right)
$$

We refer to the probabilities $P_{N, \varepsilon}\left(\Omega, \partial \Omega^{\prime}\right)$ as exit probabilities, in the sense of exiting the basin of attraction of $\Omega$, and $P_{N, \varepsilon}\left(\partial \Omega^{\prime}, \Omega^{\prime}\right)$ as contagion probabilities for the reason that when the process enters the boundary of a closed communication class, the dynamics is dominated by contagion or simply best-response.

\subsubsection{Exit probabilities}

For each pair of closed communication classes let $R\left(\Omega, \partial \Omega^{\prime}\right)$ be the number of players required to play action $x_{\Omega^{\prime}}$ by mistake for the transition $\Omega \rightarrow \partial \Omega^{\prime}$ to occur. We then write $\mathbb{P}_{i}\left(x_{\Omega^{\prime}}, \Omega\right)$ for the probability that player $i$ plays $x_{\Omega^{\prime}}$ given that the chain is in some state of $\Omega$. We also write $r\left(\Omega, \partial \Omega^{\prime}\right)=\frac{1}{N} R\left(\Omega, \partial \Omega^{\prime}\right)$ and $\mathbb{P}_{A}\left(x_{\Omega^{\prime}}, \Omega\right)=$ $\frac{1}{N} \sum_{i=1}^{N} \mathbb{P}_{i}\left(x_{\Omega^{\prime}}, \Omega\right)$. The following Lemma follows directly from Chapter 2

Proposition 4: The probability $P_{N, \varepsilon}\left(\Omega, \partial \Omega^{\prime}\right)$ of the transition from a state in $\Omega$ to another in $\partial \Omega^{\prime}$ in a single time step is bounded by

$$
\begin{aligned}
& K_{P} \exp \left\{-N\left(c\left(\Omega, \partial \Omega^{\prime}\right)+F\left(r\left(\Omega, \partial \Omega^{\prime}\right)\right)\right)\right\} \leq P_{N, \varepsilon}\left(\Omega, \partial \Omega^{\prime}\right) \\
& \leq \exp \left\{-N\left(c\left(\Omega, \partial \Omega^{\prime}\right)+F\left(r\left(\Omega, \partial \Omega^{\prime}\right)\right)\right)\right\}
\end{aligned}
$$

where $c\left(\Omega, \partial \Omega^{\prime}\right)=-r\left(\Omega, \partial \Omega^{\prime}\right) \ln \mathbb{P}_{A}\left(x_{\Omega^{\prime}}, \Omega\right), F(r)=r \ln \left(\frac{r}{1-r}\right)+\ln (1-r)$ and $K_{P}$ is some constant. 
Each $c\left(\Omega, \partial \Omega^{\prime}\right)$ is the cost of the transition $\Omega \rightarrow \partial \Omega^{\prime}$. The following example suffices to illustrate the implications of the measures contained in the bounds for exit probabilities. Consider a $2 \times 2$ coordination game with strategies $a$ and $b$ with $a$ being the risk-dominant strategy. Let the interaction structure be that of a ring network in which each player has two neighbors. There are two limit states in this case: that in which all players play $a$ and that in which they all play $b$. Since $a$ is risk-dominant, whenever a player has one neighbor playing $a$, his best-response is $a$. For $N \geq 4$, two mistakes are required to move from an all $b$ state, $\mathbf{b}$, to the basin of attraction of an all $a$ state, $\partial \mathbf{a}$. To move from a to $\partial \mathbf{b}$ requires more than $N / 2$ mistakes. We then have $r(\mathbf{b}, \partial \mathbf{a})=\frac{2}{N}$ and $r(\mathbf{a}, \partial \mathbf{b})=\frac{1}{2}$.

Now consider the case of the mistakes model of (3.2.4) in which $\varepsilon_{i}$ are independently and identically distributed across players and strategies. Let each $\varepsilon_{i}$ be drawn from a normal distribution with mean 0.05 and bounded variance. Let also $\mathcal{P}_{i}(a, \mathbf{b})=\mathcal{P}_{i}(a, \mathbf{b})=\frac{1}{2}$. That is, both strategies are equally likely whenever a mutation occurs. Then for sufficiently large $N, \mathbb{P}_{A}(b, \mathbf{a})=\mathbb{P}_{A}(a, \mathbf{b})=0.025$. We also have $F(0.5)=-0.7$ and for $N$ sufficiently large $F\left(\frac{2}{N}\right) \approx 0$.

It follows that $c(\mathbf{b}, \partial \mathbf{a})=\frac{7.4}{N}$ and $c(\mathbf{a}, \partial \mathbf{b})=1.8$. The respective bounds for the exit probabilities are $K_{P} \exp (-1.1 N) \leq P_{N, \varepsilon}(\mathbf{a}, \partial \mathbf{b}) \leq \exp (-1.1 N)$ and $6.1 \times 10^{-4} K_{P} \leq$ $P_{N, \varepsilon}(\mathbf{b}, \partial \mathbf{a}) \leq 6.1 \times 10^{-4}$. In other words, given the structure of perturbations, we can determine the rate at which exit probabilities decay with $N$. This is opposed to the case of limit noise analysis where the quantity $\ln \mathbb{P}_{A}\left(x_{\Omega^{\prime}}, \Omega\right)$ tends to negative infinity.

Condition 4 (iii) of the model definitions then implies that each $\mathbb{P}_{A}\left(x_{\Omega^{\prime}}, \Omega\right)$ does not decay with $N$ and that $\ln \mathbb{P}_{A}\left(x_{\Omega^{\prime}}, \Omega\right)$ are bounded from below for all values of N.

\subsubsection{Contagion probabilities}

This section derives bounds for contagion probabilities in terms of the parameters of the network topology and the underlying game. The characterization is based on the notion that once the chain enters the boundary of a closed communication class, it acquires a quasi-stationary distribution over the state space of a basin of attraction. The quasi-stationary distribution attained places most weight on the corresponding closed communication class. The convergence rate within a basin of attraction is 
precisely the rate at which the chain attains its quasi-stationary distribution. The following example will help illustrate this concept.

$$
P_{N, \varepsilon}=\left(\begin{array}{ccc:cccc}
0.91 & 0.05 & 0.03 & 0.003 & 0.003 & 0.004 & 0 . \\
0.85 & 0.075 & 0.07 & 0.0005 & 0.001 & 0.003 & 0.0005 \\
0.1 & 0.84 & 0.05 & 0.004 & 0.0006 & 0.0004 & 0.005 \\
\hdashline 0.0006 & 0.003 & 0.0004 & 0.015 & 0.93 & 0.044 & 0.007 \\
0.0005 & 0.0015 & 0.003 & 0.005 & 0.03 & 0.07 & 0.89 \\
0 . & 0.0002 & 0.0003 & 0.001 & 0.0005 & 0.99 & 0.008 \\
0.0001 & 0.0003 & 0.0006 & 0.009 & 0.04 & 0.9 & 0.05
\end{array}\right)
$$

Consider the Markov chain with transition matrix in (3.3.4). The state space is $\mathbf{X}=\{\mathbf{a}, \mathbf{b}, \mathbf{c}, \mathbf{d}, \mathbf{e}, \mathbf{f}, \mathbf{g}\}$. It consists of two limit states $\mathbf{a}$ and $\mathbf{f}$ and the corresponding basins of attraction are $\tilde{\mathbf{a}}=\{\mathbf{a}, \mathbf{b}, \mathbf{c}\}$ and $\tilde{\mathbf{f}}=\{\mathbf{d}, \mathbf{e}, \mathbf{f}, \mathbf{g}\}$ respectively. The basins of attraction thus form almost invariant subsets. The dashed lines partition the transition matrix into transitions within the states of almost invariant subsets (the upper left and lower right blocks of the matrix) and transitions from one invariant subset to another (upper right and lower left blocks). Whenever the process is in either of these subsets, it attains a quasi-stationary distribution that we denote by $\pi_{N, \varepsilon}(\tilde{a})$ and $\pi_{N, \varepsilon}(\tilde{f})$ respectively.

For the transition matrix (3.3.4), the respective invariant distributions rounded to the fourth decimal place are:

$$
\begin{gathered}
\pi_{N, \varepsilon}=(0.0450,0.0045,0.0021,0.0012,0.0022,0.9351,0.0995), \\
\pi_{N, \varepsilon}(\tilde{\mathbf{a}})=(0.8700,0.0865,0.0435) \text { and } \\
\pi_{N, \varepsilon}(\tilde{\mathbf{f}})=(0.0016,0.0025,0.9852,0.0107) .
\end{gathered}
$$

The process thus spends $93.5 \%$ of the time in state $\mathbf{f}$ in the long-run. When in the basin of attraction $\tilde{\mathbf{a}}$, it spend $87 \%$ of the time in state $\mathbf{a}$; when in $\tilde{\mathbf{f}}$ it spends $98.5 \%$ of the time in $\mathbf{f}$. To fully characterize the dynamics of the process within each basin of attraction, we make use of the convergence rates to the quasi-stationary distributions. Closely related to the convergence rates are the mixing times within these almost invariant subsets, the time it takes the process to attain its stationary (quasi-stationary) distribution. For each $\tilde{\Omega}$, we denote the mixing time by $T_{\tilde{\Omega}}$. For the process (3.3.4) above, the mixing time for the entire process is $T=1600$ periods, $T_{\tilde{\mathbf{a}}}=12$ periods and $T_{\tilde{\mathbf{f}}}=16$ periods.

Seneta [102] showed that the convergence rates for finite Markov chains are always exponential. That is, for a given $P_{N, \varepsilon}$ and probability mass function at time $t, \mathbf{q}_{t}$, 
$\left\|P_{N, \varepsilon}^{t} \mathbf{q}_{0}-\pi_{N, \varepsilon}\right\|=\mathcal{O}\left(\mathcal{R}^{t}\right)$, for some $\mathcal{R}<1$. We can thus generally define the convergence rate to the quasi-stationary distribution $\pi_{N, \varepsilon}(\tilde{\Omega})$ as,

$$
\mathcal{R}(\tilde{\Omega})=\limsup _{t \rightarrow T_{\tilde{\Omega}}}\left\|P_{N, \varepsilon}^{t} \mathbf{q}_{\tilde{\Omega}}-\pi_{N, \varepsilon}(\tilde{\Omega})\right\|^{\frac{1}{t}}
$$

where $\mathbf{q}_{\tilde{\Omega}}$ is the probability mass function that places most weight on some initial state of the chain in $\tilde{\Omega}$.

The contagion probabilities (for transitions $\partial \Omega \rightarrow \Omega$ ) are then proportional to $1-\mathcal{R}(\tilde{\Omega})$. This is based on the fact that since $\mathcal{R}(\tilde{\Omega})<1$, the closer $\mathcal{R}(\tilde{\Omega})$ is to one the longer the process takes to converge to its quasi-stationary distribution. We can then write $P_{N, \varepsilon}(\partial \Omega, \Omega)=(1-\mathcal{R}(\tilde{\Omega}))^{K_{\Omega}}$, where $K_{\Omega}$ is some constant. Equivalently,

$$
P_{N, \varepsilon}(\partial \Omega, \Omega)=\exp \left(K_{\Omega} \ln (1-\mathcal{R}(\tilde{\Omega}))\right)
$$

The parameters $\mathcal{R}(\tilde{\Omega})$ are equivalent to the second largest eigenvalues of the block matrices describing the transitions within the states of almost invariant subsets (see proof of Proposition 5 below). For the process (3.3.4) the respective values are $\mathcal{R}(\tilde{\mathbf{a}})=$ 0.2002 and $\mathcal{R}(\tilde{\mathbf{f}})=0.2851$. Hence $P_{N, \varepsilon}(\partial \mathbf{a}, \mathbf{a})=\exp \left(-0.22 K_{\Omega}\right)$ and $P_{N, \varepsilon}(\partial \mathbf{f}, \mathbf{f})=$ $\exp \left(-0.34 K_{\Omega}\right)$.

In what follows, we derive and expression for $\mathcal{R}(\tilde{\Omega})$ in terms of computable parameters rather than using its relation to the eigenspectrum of the entire transition matrix. Here, we provide bounds in terms of the eigenspectrum of the network of interactions. We begin with the following definitions.

Denote the normalized adjacency matrix associated with the network of interactions by $\mathscr{A}$ (we omit the index $N$ for notational simplicity). Let also $\rho(\mathscr{A})=$ $\left(\lambda_{1}^{N}, \cdots, \lambda_{N}^{N}\right)$ be its eigenvalue spectrum ordered in such away that $\lambda_{1}^{N}=1 \geq \lambda_{2}^{N} \geq$ $\cdots \geq \lambda_{N}^{N}$. Denote by $\Sigma_{\varepsilon}$ for a player's individual transition matrix given an opponent's actions. That is let $\mathbb{P}\left(x_{j} \mid x_{i}\right)$ be the probability that a given player plays action $x_{j} \in X$ given that his opponent is playing $x_{i} \in X$ in the current period. Then $\Sigma_{\varepsilon}$ is given by

$$
\Sigma_{\varepsilon}=\left(\begin{array}{cccc}
\mathbb{P}\left(x_{1} \mid x_{1}\right) & \mathbb{P}\left(x_{2} \mid x_{1}\right) & \cdots & \mathbb{P}\left(x_{m} \mid x_{1}\right) \\
\mathbb{P}\left(x_{1} \mid x_{2}\right) & \mathbb{P}\left(x_{2} \mid x_{2}\right) & \cdots & \mathbb{P}\left(x_{m} \mid x_{2}\right) \\
\vdots & \vdots & \ddots & \vdots \\
\mathbb{P}\left(x_{1} \mid x_{m}\right) & \mathbb{P}\left(x_{2} \mid x_{m}\right) & \cdots & \mathbb{P}\left(x_{m} \mid x_{m}\right)
\end{array}\right)
$$

Let also $\rho\left(\Sigma_{\varepsilon}\right)=\left(\vartheta_{1}, \cdots, \vartheta_{m}\right)$ be the eigenvalue spectrum of $\Sigma_{\varepsilon}$. The following Proposition provides bounds for convergence rates. 
Proposition 5: For a given network of interactions $G_{N}$, the convergence rate $\mathcal{R}(\tilde{\Omega})$ within a basin of attraction has the following bounds

$$
1-\lambda_{2}^{N}(G) \leq 1-\mathcal{R}(\tilde{\Omega}) \leq 1-\vartheta_{m} \lambda_{2}^{N}(G)
$$

where $\lambda_{2}^{N}(G)$ is the second largest eigenvalue of the normalized adjacency matrix of network $G_{N}$.

Proof. See Appendix 3.8.2

The eigenvalues of $\Sigma_{\varepsilon}, \vartheta_{m}$ are functions of the underlying payoff structure and the level of noise. As the noise level tends to zero, $\vartheta_{m}$ tend to one.The quantity $1-\lambda_{2}^{N}(G)$ is also generally referred to as the spectral gap of $G_{N}$. A bound on $\lambda_{2}^{N}$ can be established through its relationship with the graph conductance $\phi(G)$. We give examples for specific graphs below.

EXAMPLE: The following relation between second the eigenvalue of a network graph $G_{N}, \lambda_{2}^{N}(G)$ and $\phi(G)$ can be derived from Cheeger inequality: See Appendix 3.8.3 for more detail concerning the relation plus derivations for the following examples.

$$
\lambda_{2}^{N}(G) \leq 1-\frac{\phi(G)^{2}}{2}
$$

(i) Complete network $\left(G_{c o m}\right)$ : a network structure in which every vertex is connected to every other vertex: $\lambda_{2}^{N}\left(G_{\text {com }}\right) \leq \frac{7}{8}$.

(ii) $1-D$ cyclic network $\left(G_{c y c}\right)$ : a network in which vertices are arranged in a circle and every vertex is connected to two other neighboring vertices; $\lambda_{2}^{N}\left(G_{c y c}\right) \leq$ $\frac{N^{2}-2}{N^{2}}$.

(iii) $2 D N \times N$ lattice network $\left(G_{2 D}\right)$ : a lattice structure constructed with periodic boundary conditions such that each agent is connected to 4 neighbors: $\lambda_{2}^{N}\left(G_{2 D}\right) \leq \frac{16 N^{2}-1}{16 N^{2}}$

(iv) Random $d$-regular network $\left(G_{d-r}\right)$ : a network structure in which each of the $N$ vertices is connected to $d$ other vertices chosen at random: $\lambda_{2}^{N}\left(G_{d-r}\right) \leq \frac{7}{8}$.

(v) Newman's small world network $\left(G_{n s w}\right)$ : a network structure in which the mean shortest-path between nodes increases sufficiently slowly (logarithmically) as a function of the number of nodes in the network: $\lambda_{2}^{N}\left(G_{n s w}\right)=1-\mathcal{O}\left(\frac{c}{(\ln N)^{2}}\right)$, where $c$ is a constant. 
In general, densely connected and random networks have higher second largest eigenvalues compared to sparsely connected network. For sufficiently small noise, $\vartheta_{m}$ is close to one such that $1-\mathcal{R}(\tilde{\Omega})$ is approximately equal to $1-\lambda_{2}^{N}(G)$. This enables us to focus on the influence of the network on long-run stability. Propositions 4 and 5 then yield the following Proposition.

Proposition 6: For a given network of interactions $G_{N}$ with the second eigenvalue of its normalized adjacency matrix $\lambda_{2}^{N}(G)$, and some constant $0 \leq K_{\Omega} \leq 1$ such that $\gamma^{N}(\Omega)=$ $K_{\Omega} \ln \left(1-\lambda_{2}^{N}(G)\right)$, the probability of the transition $\Omega \rightarrow \Omega^{\prime}$ is bounded by

$$
\begin{aligned}
& K_{F} \exp \left\{-N\left(c\left(\Omega, \partial \Omega^{\prime}\right)+F\left(r\left(\Omega, \partial \Omega^{\prime}\right)\right)\right)+\gamma^{N}\left(\Omega^{\prime}\right)\right\} \leq P_{N, \varepsilon}\left(\Omega, \Omega^{\prime}\right) \\
& \leq \exp \left\{-N\left(c\left(\Omega, \partial \Omega^{\prime}\right)+F\left(r\left(\Omega, \partial \Omega^{\prime}\right)\right)\right)+\gamma^{N}\left(\Omega^{\prime}\right)\right\}
\end{aligned}
$$

where $c\left(\Omega, \partial \Omega^{\prime}\right)=-r\left(\Omega, \partial \Omega^{\prime}\right) \ln \mathbb{P}_{A}\left(x_{\Omega^{\prime}}, \Omega\right), F(r)=r \ln \left(\frac{r}{1-r}\right)+\ln (1-r)$ and $K_{F}$ is some constant.

The following shorthand and definitions are used for the remainder of the Chapter. First, for a given $N$ we write $\psi^{N}\left(\Omega, \Omega^{\prime}\right)=N\left(c\left(\Omega, \partial \Omega^{\prime}\right)+F\left(r\left(\Omega, \partial \Omega^{\prime}\right)\right)\right)-\gamma^{N}\left(\Omega^{\prime}\right)$, $\psi_{\min }^{N}(\Omega)=\min _{\Omega^{\prime} \neq \Omega} \psi^{N}\left(\Omega, \Omega^{\prime}\right)$ and $\psi_{\max }^{N}(\Omega)=\max _{\Omega^{\prime} \neq \Omega} \psi^{N}\left(\Omega^{\prime}, \Omega\right)$. And $\psi^{N}(\Omega)=$ $\psi_{\max }^{N}(\Omega)-\psi_{\min }^{N}(\Omega)$. Secondly, we define the paths in relation to the expression in (3.3.2). Let $\mathscr{H}_{\Omega_{1} \Omega_{J}}$ be the set of all directed paths starting from $\Omega_{1}$ and terminating at $\Omega_{J}$, and let $H_{\Omega_{1} \Omega_{J}}=\left(\Omega_{1}, \cdots, \Omega_{j}, \cdots, \Omega_{J}\right)$ be the typical path in $\mathscr{H}_{\Omega_{1} \Omega_{J}}$. Then the path potential $\xi^{N}\left(H_{\Omega_{1} \Omega_{J}}\right)$ of $H_{\Omega_{1} \Omega_{J}}$ is defined as

$$
\xi^{N}\left(H_{\Omega_{1} \Omega_{J}}\right)=\sum_{j=1}^{J-1}\left(\psi^{N}\left(\Omega_{j}, \Omega_{j+1}\right)-\psi_{\min }^{N}\left(\Omega_{j+1}\right)\right)
$$

That is, the total cost of the path minus the total of minimum deviations from the path. The path potential is thus a measure of how accessible or reachable a given closed communication class is from another through that particular path. The logic behind the definition is that the accessibility of $\Omega_{J}$ from $\Omega_{1}$ through $H_{\Omega_{1} \Omega_{J}}$ depends on the total cost associated with $H_{\Omega_{1} \Omega_{J}}$ and the likelihood of deviating from $H_{\Omega_{1} \Omega_{J}}$. The higher the cost the more difficult it is to reach $\Omega_{J}$ through $H_{\Omega_{1} \Omega_{J}}$ and the lower the resistance of the basins of attraction that the path traverses to reach $\Omega_{J}$ the more likely that the process will follow such a path. We can also write the equivalent limit quantities as $\psi^{L}(\Omega)=\lim _{N \rightarrow \infty} \frac{1}{N} \psi^{N}(\Omega)$, and

$$
\xi^{L}\left(H_{\Omega_{1} \Omega_{J}}\right)=\lim _{N \rightarrow \infty} \frac{1}{N} \xi^{N}\left(H_{\Omega_{1} \Omega_{J}}\right)
$$

We then define the maximum path potential of any given closed communication class $\Omega_{J}$ as follows

$$
\xi^{N}\left(\Omega_{J}\right)=\max _{\Omega_{j} \neq \Omega_{J}} \min _{H_{\Omega_{j} \Omega_{J}} \in \mathscr{H}_{\Omega_{j} \Omega_{J}}} \xi^{N}\left(H_{\Omega_{j} \Omega_{J}}\right)
$$


Given these definitions, the following proposition holds.

Proposition 7: If $\Omega^{*}$ is the asymptotically globally stable set, then

(i) $\psi^{L}\left(\Omega^{*}\right)<0$,

(ii) $\xi^{L}\left(\Omega^{*}\right)<0$ and

Proof. See Appendix 3.8.4.

The quantities $\psi^{L}\left(\Omega^{*}\right)$ and $\xi^{L}\left(\Omega^{*}\right)$ are closely related to the notion of radius, coradius and modified co-radius in Ellison [38]. More specifically, each $\psi^{N}(\Omega)$ is related to the difference between co-radius and radius of $\Omega$ and $\xi^{N}(\Omega)$ is related to the difference between the modified co-radius and radius of $\Omega$. The manner in which we make use of these measures is however different in that we consider their values at the limit of $N$. That is $\psi^{L}\left(\Omega^{*}\right)$ and $\xi^{L}\left(\Omega^{*}\right)$. Similarly, because we keep the noise level positive, we do not talk about the radius, co-radius and modified co-radius but rather the cost of direct transitions and costs of paths between limit sets. The conditions are identical in that in both cases what matters is whether or not these quantities are negative. We now relate the conditions in Proposition 7 to the parameters of network topology and properties of the payoff structure. The following theorem is a first step towards this goal.

THEOREM 2: Given a family of networks $G_{N}$, a subset $\Omega^{*}$ is asymptotically globally stable if

(i) $\lim _{N \rightarrow \infty} \frac{1}{N} \ln \left(1-\lambda_{2}^{N}(G)\right)=0$,

(ii) $\lim _{N \rightarrow \infty} r\left(\Omega_{j}, \partial \Omega^{*}\right)=0$ for all $\Omega_{j} \neq \Omega^{*}$, or

(ii) if for all $\Omega_{j} \neq \Omega^{*}$, there exists a directed path $H_{\Omega_{j} \Omega^{*}}$ such that for each ordered pair $\left(\Omega_{j_{1}}, \Omega_{j_{2}}\right) \in H_{\Omega_{j} \Omega^{*}}, \lim _{N \rightarrow \infty} r\left(\Omega_{j_{1}}, \Omega_{j_{2}}\right)=0$ but $\lim _{N \rightarrow \infty} r\left(\Omega^{*}, \Omega_{j}\right)>0$

Proof. See Appendix 3.8.5

Theorem 2 provides conditions on the interaction structure and the underlying base game for which asymptotic global convergence is feasible. This result is clearly independent of the model of mistakes. This is because the relevant quantities in the computation of asymptotically globally stable sets are $r\left(\Omega, \partial \Omega^{\prime}\right)$ and the probabilities of mistakes, captured by $\ln \left(\mathbb{P}_{A}\left(x_{\Omega^{\prime}}, \Omega\right)\right)$, are negligible. Provided that the noise levels are positive. The first sufficient condition for asymptotic global convergence is that the rate at which the spectral gap of the interaction network grows with $N$ must 
be negligible at the limit of $N$. This condition is valid for most strongly connected network structures. By strongly connected we mean those cases in which there exists a directed path from any one player to any other player. Consider the examples subsection 3.3.2 above. For the case of random $d$-regular network $\left(G_{d-r}\right)$, where $\lambda_{2}^{N}\left(G_{d-r}\right) \leq$ $\frac{7}{8}$ we have $\ln \left(1-\lambda_{2}^{N}\left(G_{d-r}\right)\right)=\ln \left(\frac{1}{8}\right)$. Thus $\lim _{N \rightarrow \infty} \frac{1}{N} \ln \left(1-\lambda_{2}^{N}\left(G_{d-r}\right)\right)=0$. Similarly, for the $2 D N \times N$ lattice network $\left(G_{2 D}\right)$, where $\lambda_{2}^{N}\left(G_{2 D}\right) \leq \frac{16 N^{2}-1}{16 N^{2}}$, we have

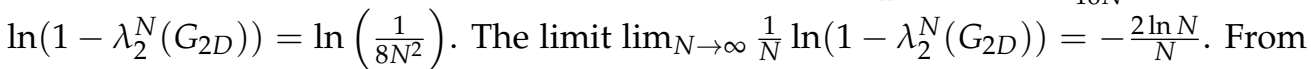
L'Hôpital's rule it follows that $\lim _{N \rightarrow \infty} \frac{1}{N} \ln \left(1-\lambda_{2}^{N}\left(G_{2 D}\right)\right)=0$.

Conditions ( $\mathrm{ii}$ ) and (iii) satisfy what we refer to as global and path-wise contagion respectively. We discuss each of these notions in the following sections. As we define below, a strategy is contagious relative to another for a given payoff and network it can spread by best-response once it has been adopted by a small fraction of the population. This property determines whether or not the quantities $r\left(\Omega, \partial \Omega^{\prime}\right)$ are decreasing functions of $N$. If a strategy played by all or a majority of players in $\Omega^{\prime}$ is contagious relative to that played in $\Omega$ then $r\left(\Omega, \partial \Omega^{\prime}\right)$ will be a decreasing function of $N$, and vice versa. Based on this argument, we then introduce the concepts of global and path-wise contagion in relation to conditions in Theorem 2 (ii) and (iii) respectively. We then show that actions that are globally and/or path-wise contagions are those that are asymptotically globally stable.

\subsection{GLOBAL CONTAGION}

Global contagion, in a literal sense implies that there exists a unique strategy for a given network structure, that can spread through best-response once a small fraction of the population has adopted it. More formally, given any pair of closed communication classes $\Omega$ and $\Omega^{\prime}$, let $x_{\Omega}$ and $x_{\Omega^{\prime}}$ be the strategies played by all or the majority of players in each respective class. $R\left(\Omega, \partial \Omega^{\prime}\right)$ is the number of players required to play $x_{\Omega^{\prime}}$ to trigger contagion. Then,

DeFinition 6: Let $|\mathbf{x}|_{a}$ denote the number of players playing strategy a in state $\mathbf{x}$. For the transition $\Omega \rightarrow \Omega^{\prime}, x_{\Omega^{\prime}}$ is said to be contagious relative to $x_{\Omega}$ if $x_{\Omega^{\prime}}$ spreads by best-response whenever $|\mathbf{x}|_{x_{\Omega^{\prime}}}>R\left(\Omega, \partial \Omega^{\prime}\right)$. The strategy $x_{\Omega^{\prime}}$ is globally contagious if it is contagious for all transitions $\Omega \rightarrow \Omega^{\prime}$ for all $\Omega \neq \Omega^{\prime}$.

That is, a strategy $x_{\Omega^{\prime}}$ is contagious if it spreads by best-response once at least $R\left(\Omega, \partial \Omega^{\prime}\right)$ of the players have adopted it. The feasibility of global contagion depends on the properties of the interaction network and the payoff structure: That is contagion threshold and relative payoff gains respectively. 
The relative payoff gain denoted by $\eta_{a b}$, for any pair of strategies $a$ and $b$ is defined by

$$
\eta_{b a}=\frac{U(b, b)-U(a, b)}{U(b, b)-U(b, a)+U(a, a)-U(a, b)}
$$

and $\eta_{a b}=1-\eta_{b a}$. The quantities $U(b, b)-U(a, b)$ and $U(a, a)-U(a, b)$ are the private and social payoff gain of switching from $b$ to $a$ respectively. The measure of relative payoff gain $\eta_{b a}$, can also be interpreted as the fraction of neighbors that each player requires to play $a$ for that player to switch from $b$ to $a$ through best-response, and vice versa for $\eta_{a b}$. We write $\lceil m\rceil$ for the smallest integer not less than $m$ such that for each $i \in \mathcal{N}$ with respective degree $k_{i}$, the quantity $\left\lceil\eta_{b a} k_{i}\right\rceil$ is the minimum number of neighbors $i$ requires to play $a$ for $i$ to do likewise.

For every interaction network $G_{N}$ there exists a real number $0<\eta_{G}<1$ such that for any pair of strategies say $a$ and $b$, strategy $a$ is contagious relative to $b$ whenever $\eta_{b a} \leq \eta_{G}$. We then say that a strategy $a$ is globally contagious under interaction network $G_{N}$ whenever $\eta_{b a} \leq \eta_{G}$ for all $b \neq a$. Or equivalently, whenever $\eta_{\max , a} \leq \eta_{G}$, where $\eta_{\max , a}=\max _{b \neq a} \eta_{b a}$. This real number $\eta_{G}$ is the contagion threshold of network $G_{N}$ [78].

Every arbitrary network of interactions has a unique contagion threshold. Consider for the example the round-robin network structure in which each player $i$ has two neighbors $i-1$ and $i+1$. Such a network structure has the contagion threshold of $\frac{1}{2}$. Implying that for any underlying base game containing a unique strategy say $a$ with maximum relative payoff gain $\eta_{\max , a}<\frac{1}{2}$ (that is a risk-dominant strategy), such a strategy is globally contagious under the round-robin network structure. Morris [78] provides an explicit characterization of contagion threshold for various families of deterministic networks. Lelarge [68] derives similar conditions for random networks.

The above definition of global contagion is related to the notion of $p$-dominance according to Morris et al. [79]. An action pair in a two-player game is said to be $p$-dominant if each action is a best response to any belief that the other player takes the action in this pair with probability at least $p$. For the local interaction game we consider, a strategy is $p$-dominant if for all players, it is the unique best-response when it is played by at least proportion $p$ of the neighbors. This implies that for a network with contagion threshold $p$, a strategy that is $p$-dominant is also globally contagious.

The following example illustrate the selection of globally contagious strategies for given network structures. 


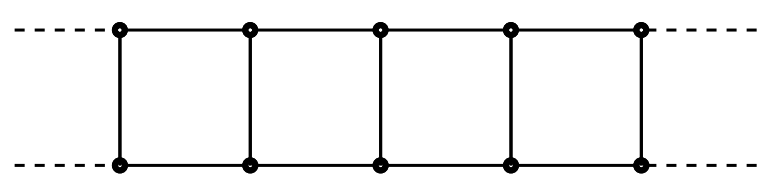

Figure 2: The 2-dimensional network with contagion threshold of $\frac{1}{3}$.

Example 3. For the $3 \times 3$ game of Table 5, strategy $b$ is globally contagious and hence asymptotically globally stable for all networks with contagion threshold $\eta_{G} \geq \frac{1}{3}$. This is true irrespective of the model of mistakes.

Table 5: For any pair of players the profile $(b, b)$ is risk-dominant.

a

\begin{tabular}{|c|c|c|}
\hline \multicolumn{1}{c|}{$\mathrm{a}$} & $\mathrm{b}$ & $\mathrm{c}$ \\
\hline 6,6 & 0,5 & 0,0 \\
\hline 5,0 & 7,7 & 6,3 \\
\hline 0,0 & 3,6 & 8,8 \\
\hline
\end{tabular}

Proof. The proof follows by first determining the relative payoff gains for each pair of strategies. We have $\eta_{a b}=\frac{1}{8} . \eta_{b a}=\frac{7}{8}, \eta_{b c}=\frac{2}{3}, \eta_{c b}=\frac{1}{3}, \eta_{a c}=\frac{5}{8}, \eta_{c a}=\frac{5}{6}$. It follows that $\eta_{\max , a}=\frac{7}{8}, \eta_{\max , b}=\frac{1}{3}$ and $\eta_{\max , c}=\frac{2}{3}$. Hence strategy $b$ is uniquely globally stable for all network structures with contagion threshold $\eta_{G} \geq \frac{1}{3}$.

Example 3 demonstrates the selection of globally contagious strategies and more specifically that there exists a range of families of networks for which global contagion and hence asymptotic global convergence is feasible. In this example, global contagion is feasible in all networks whose contagion threshold is $\frac{1}{3} \leq \eta_{G} \leq \frac{1}{2}$. For example the network in Figure 2.

Now, consider the $n$-dimensions $l$-max distance interactions family of networks. That is given an $n$-dimensional lattice network, each player interacts with all players who are within the distance of $l$ steps away in all directions. See Figure 9 for the case of $n=2$ and $l=1$. Morris [78] provides general expressions for contagion thresholds for this family of networks. For example when $n=2$ the contagion thresholds are 
given by $\eta_{G}=\frac{l(2 l+1)}{(2 l+1)^{2}-1}$ for $l=1,2,3, \cdots$. For $l=1$ we have $\eta_{G}=\frac{3}{8}$. For this family of interaction networks, that is for all values of $n$ and $l$, the contagion threshold is $\eta_{G} \geq \frac{1}{3}$, implying global contagion of strategy $b$.

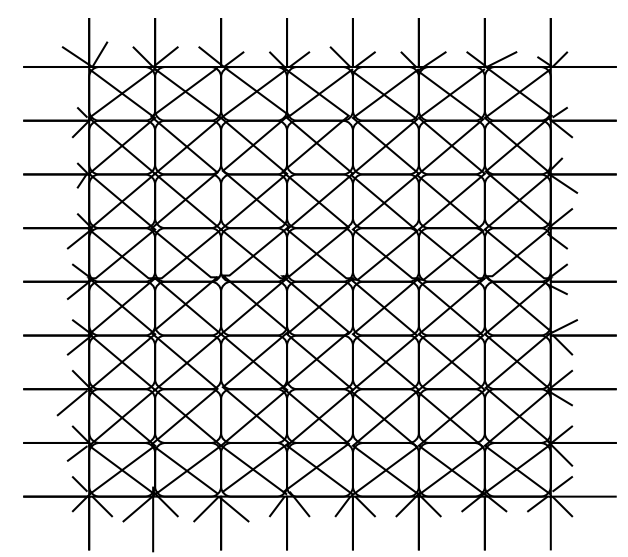

Figure 3: The 2-dimensions 1-max distance interactions.

\subsection{PATH-WISE CONTAGION}

Path-wise contagion, in a literal sense, means that there exists a directed path between a pair of closed communication classes whereby the strategies played in each directed relation is contagious but not vice versa. Formally, path-wise contagion is defined as follows.

Definition 7: Consider any pair of closed communication classes $\Omega_{1}$ and $\Omega_{J}$ with the directed path $H_{\Omega_{1} \Omega_{J}}=\left\{\Omega_{1} \rightarrow \cdots \rightarrow \Omega_{j} \rightarrow \cdots \rightarrow \Omega_{J}\right\}$. Let $x_{\Omega_{j}}$ be the strategy played by the majority of players in some state of $\Omega_{j}$. Then for a given interaction network $G_{N}, \Omega_{J}$ and hence $x_{\Omega_{J}}$ is said to be path-wise contagious relative to $\Omega_{1}$ if each $\eta_{x_{\Omega_{j}} x_{\Omega_{j+1}}} \leq \eta_{G}$ but $\eta_{\Omega_{\Omega_{j+1}} x_{\Omega_{j}}}>\eta_{G}$ and that $\eta_{x_{\Omega_{j}} x_{\Omega_{1}}}>\eta_{G}$.

This means that, for every pair of strategies say $a$ and $d$ with directed path relations $a \rightarrow b \rightarrow c \rightarrow d$, strategy $d$ is path-wise contagious relative to $a$ if $b$ is contagious relative to $a, c$ is contagious relative to $b$ and $d$ is contagious relative to $c$, but not vice versa. We provide two examples to illustrate the concept of path-wise contagion. The first example demonstrates how the concept of path-wise contagion can be employed to derive global convergence to risk-dominant strategies in the absence of global contagion. The second example shows that even in the absence of a unique risk- 
dominant strategy, one can still design an interaction network in which asymptotic global convergence occurs.

Example 4. For the $3 \times 3$ game of Table 6, strategy $c$ is path-wise contagious and hence asymptotically globally stable for all network structures.

Table 6: For any pair of players the profile $(c, c)$ is risk-dominant.

\begin{tabular}{c|c|c|c|}
\multicolumn{1}{c}{} & \multicolumn{1}{c}{$\mathrm{a}$} & $\mathrm{b}$ & $\mathrm{c}$ \\
\cline { 2 - 4 } $\mathrm{a}$ & 6,6 & 0,5 & 0,0 \\
\hline $\mathrm{b}$ & 5,0 & 7,7 & 5,5 \\
\hline $\mathrm{c}$ & 0,0 & 5,5 & 8,8 \\
\hline
\end{tabular}

Proof. The relative payoff gains for each pair of strategies are $\eta_{a b}=\frac{1}{8} \cdot \eta_{b a}=\frac{7}{8}, \eta_{b c}=$ $\frac{2}{5}, \eta_{c b}=\frac{3}{5}, \eta_{a c}=\frac{5}{8}, \eta_{c a}=\frac{5}{6}$. It follows that $\eta_{\text {max }, a}=\frac{7}{8}, \eta_{\text {max }, b}=\frac{3}{5}$ and $\eta_{\text {max }, c}=\frac{5}{8}$. Since the maximum possible contagion threshold for any network structure is $\frac{1}{2}$, it follows that no single strategy is globally contagious for all network structures.

First consider the case of network structures with contagion threshold of $\eta_{G} \geq \frac{2}{5}$. For such networks $b$ is contagious relative to $a$ and $c$ is contagious relative to $b$ and not vice versa. The only path that is path-wise contagious is $H_{\mathbf{a c}}=\{\mathbf{a} \rightarrow \mathbf{b} \rightarrow \mathbf{c}\}$. Implying that $\mathbf{c}$ (and hence strategy $c$ ) is the asymptotically globally stable state (strategy) in such network families.

Now, consider the case of network structures with contagion threshold of $\eta_{G} \geq \frac{1}{8}$. For such networks $b$ is contagious relative to $a$ but $c$ is no longer contagious relative $b$ since $\eta_{b c}>\eta_{G}$. There however exist a series of intermediate limit states between b and $\mathbf{c}$ induced by the network structure. These states can be ordered in such a way that they are contagious relative to each other. There exists a directed path $H_{\mathbf{b c}}=\left\{\mathbf{b} \rightarrow \cdots \rightarrow \Omega_{j} \cdots \rightarrow \cdots \rightarrow \mathbf{c}\right\}$. Where $\Omega_{j}$ 's are the intermediate limit states. Consider the 2-dimensions nearest neighbor interactions network of Figure 4 for example. In this network structure, the intermediate states involve two players in each enclave of four simultaneously switching from playing $b$ to playing $c$. More 
explicitly, if we consider the diagonal enclaves, then for an $N$ size network we have $\sqrt{N}$ of such limit states, each with $r\left(\Omega_{j}, \Omega_{j+1}\right)=\frac{2}{N}$.

$$
\xi\left(H_{\mathbf{b c}}\right)=\sum_{j=1}^{\sqrt{N}}\left\{\frac{2}{N}-r_{\min }\left(\Omega_{j+1}\right)\right\}=\frac{2}{\sqrt{N}}-\sum_{j=1}^{\sqrt{N}} r_{\min }\left(\Omega_{j+1}\right)
$$

Since $r(\mathbf{a}, \mathbf{b})=\frac{1}{N}$ and $r(\mathbf{b}, \mathbf{a})=\mathcal{O}(N)$, we then have

$$
\xi\left(H_{\mathrm{ac}}\right)=\left(\frac{1}{N}+\frac{2}{\sqrt{N}}\right)-\left(\mathcal{O}(N)+\sum_{j=1}^{\sqrt{N}} r_{\min }\left(\Omega_{j+1}\right)\right)
$$

Hence at the limit of $N, \xi\left(H_{\mathbf{a c}}\right)<0$, and $\mathbf{c}$ is the asymptotically globally stable state under such families of networks.

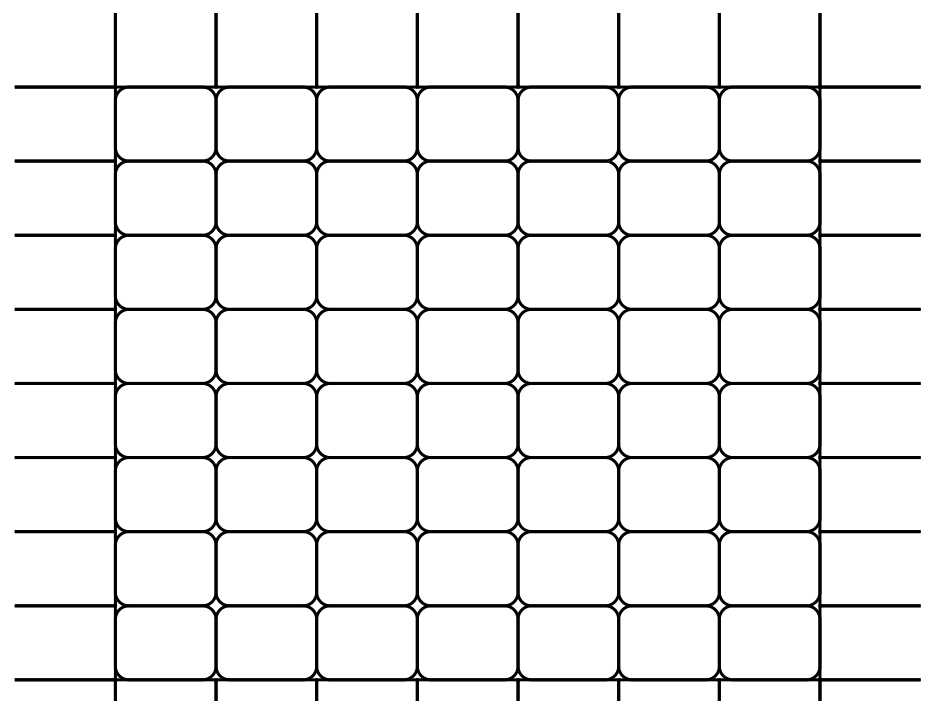

Figure 4: The 2-dimensions nearest neighbor interactions network.

For the case of networks with $0<\eta_{G} \leq \frac{1}{8}$, the similar argument of intermediate limit states that applies between $\mathbf{b}$ and $\mathbf{c}$ will apply between $\mathbf{a}$ and $\mathbf{b}$. Making $\mathbf{c}$ the asymptotically stable state in this family of networks as well.

Example 4 acts to illustrate one of our main results of Theorem 2 that if the underlying base game contains a unique risk-dominant strategy, then it is always selected independently of the model of mistakes and network structure. This result generalizes earlier results by Lee and Valentinyi [67] and Lee et al. [66] to $m \times m$ matrix games, models of mistakes and to arbitrary networks. The result is contrary to the 
case of global interactions that crucially depends on the model of mistakes. For example Young [113] showed that for the game in Table 6 the stochastically stable strategy is $b$ under global interaction and mistakes model. Under multinomial logit model however, strategy $c$ is stochastically stable. The main reason for the difference is that under global interactions, what matters for stochastic stability is simply the number of other players that must switch to a different strategy for any given player to do likewise. For example, in the game of Table 6 for any given player to switch from $c$ to $b$, at least $\frac{3}{8}$ of the other players must play $a$. Since every player observes every other player's strategy, this process needs to occur only once. Under local interaction however, even if $\frac{3}{8}$ of any players neighbors switched to playing $a$, only that one player would switch to playing $b$ and every other player keeps playing $c$. Moreover, over time the process can easily revert back to an all $c$ state. In other words, what matters is whether or not the mistakes that occur can cause some form of relative contagion.

Example 5. For the $3 \times 3$ game of Table 7 , strategy $b$ is path-wise contagious and hence asymptotically globally stable for network structures with $\eta_{G}=\frac{1}{3}$, such as that in Figure 2.

Table 7: In this game, no strategy is uniquely risk-dominant. Strategy a riskdominates $b, b$ risk-dominates $c$ and $c$ risk-dominates $a$

a

\begin{tabular}{|c|c|c|}
\hline \multicolumn{1}{c}{$a$} & $\mathrm{~b}$ & $\mathrm{c}$ \\
\hline 5,5 & 3,1 & 0,3 \\
\hline 1,3 & 6,6 & 2,2 \\
\hline 3,0 & 2,2 & 4,4 \\
\hline
\end{tabular}

Proof. The relative payoff gains for each pair of strategies are $\eta_{a b}=\frac{4}{7}, \eta_{b a}=\frac{3}{7}$, $\eta_{b c}=\frac{2}{3}, \eta_{c b}=\frac{1}{3}, \eta_{a c}=\frac{1}{3}, \eta_{c a}=\frac{2}{3}$. It follows that $\eta_{\max , a}=\frac{2}{3}, \eta_{\max , b}=\frac{4}{7}$ and $\eta_{\max , c}=\frac{2}{3}$. Implying that no single strategy is globally stable for all network structures. For $\eta_{G}=\frac{1}{3}$ however, $c$ is contagious relative to $a$ and $b$ is contagious relative to $c$, such that $H_{\mathbf{a b}}=\{\mathbf{a} \rightarrow \mathbf{c} \rightarrow \mathbf{b}\}$ is path-wise contagious. Hence, strategy $b$ is asymptotically globally stable for this family of networks. It is then easy to check that for all networks with $\eta_{G}>\frac{1}{3}$, all strategies are contagious relative to each other and hence global convergence does not occur. Similarly, for $\eta_{G}<\frac{1}{3}$ the network structure induces intermediate limit states between $\mathbf{a}$ and $\mathbf{c}, \mathbf{c}$ and $\mathbf{b}$, and $\mathbf{b}$ and $\mathbf{a}$, equally leading to a cycle among the limit states. 
Example 5 acts to illustrate one of the main implications of Theorem 2, which is that for any given underlying game, it is possible to construct a network structure for which a unique strategy is globally stable provided that the relative payoff gains are not equivalent for all strategies. Strategy $b$ is globally stable under the network structure in Figure 2 for example. For any network structure with contagion threshold $\eta_{G}>\frac{1}{3}$ or $\eta_{G}<\frac{1}{3}$, all the three strategies are almost equally likely in the long-run. This result is quite strong and even more when viewed in reverse. That is, for a given network structure and set of strategies, it is possible to construct a payoff matrix hence relative payoff gains for which a given strategy gets globally adopted in the long-run. Moreover, the result is independent of the model of mistakes.

The final note we make in this section is that asymptotic global stability is robust to addition of strictly dominated strategies. This criticism of stochastic stability as a solution concept was pointed out by [59]. Global and path-wise contagion (hence asymptotic global stability) are however robust to this phenomenon. Addition of strictly dominated strategies does not make a strategy that was originally globally or path-wise contagious non-contagious. Similarly, addition of strictly dominated strategies does not make strategies that are not contagious contagious.

Consider the game in Table 8 for example. The relative payoff gains are $\eta_{a b}=$ $\frac{6}{13}, \eta_{b a}=\frac{7}{13}$, and hence under global interactions we have that $r(\mathbf{a}, \partial \mathbf{b})=\frac{6}{13}$ and $r(\mathbf{b}, \partial \mathbf{a})=\frac{7}{13}$. It can then easily be checked that $\mathbf{b}$ is stochastically stable under both the mistakes model with state independent mutations and the multinomial logit model. Similarly, since $b$ is either globally or path-wise contagious relative to $a$, it is asymptotically globally stable for all network structures.

Table 8: Profile $(b, b)$ is risk-dominant.

\begin{tabular}{c|c|c|}
\multicolumn{1}{c}{$\mathrm{a}$} & $\mathrm{b}$ \\
\cline { 2 - 3 } $\mathrm{a}$ & 8,8 & 5,5 \\
\hline $\mathrm{b}$ & 5,5 & $8.5,8.5$ \\
\hline
\end{tabular}

Now, consider the game in Table 9 derived from the game in Table 8 by addition of a strictly dominated strategy $c$. Under global interactions, $r(\mathbf{a}, \partial \mathbf{b})=\frac{6}{13}$ and $r(\mathbf{b}, \partial \mathbf{a})=\frac{1}{3}$, which means that a becomes stochastically stable. The addition of 
a strictly dominated strategy however does not affect the relative payoff gains and hence the values of $r(\mathbf{a}, \partial \mathbf{b})$ and $r(\mathbf{b}, \partial \mathbf{a})$ for any given network structure.

Table 9: Game in Table 8 with addition of strictly dominated strategy $c$.

a

\begin{tabular}{|c|c|c|}
\hline \multicolumn{1}{c}{$\mathrm{a}$} & $\mathrm{b}$ & $\mathrm{c}$ \\
\hline 8,8 & 5,5 & 7,7 \\
\hline 5,5 & $8.5,8.5$ & 0,6 \\
\hline 7,7 & 6,0 & 0,0 \\
\hline
\end{tabular}

\subsection{OTHER EXAMPLES}

The following two examples act to illustrate the case in which more than one strategy and the globally pairwise risk-dominant strategy may or may not be asymptotically globally stable. The game in Table 11 is adopted from Alós-Ferrer and Weidenholzer [8] and that in Table 10 is derived from the former by deleting strategy $d$.

Table 10: Strategy $a$ is globally pairwise risk-dominant and $0<\epsilon<\frac{1}{5}$.

\begin{tabular}{l|c|c|c|}
\multicolumn{1}{c}{} & \multicolumn{1}{c}{$\mathrm{a}$} & $\mathrm{b}$ & $\mathrm{c}$ \\
\cline { 2 - 4 } $\mathrm{a}$ & 0 & 2 & 2 \\
\cline { 2 - 4 } $\mathrm{b}$ & $-2+2 \epsilon$ & $3+2 \epsilon$ & 4 \\
\cline { 2 - 4 } $\mathrm{c}$ & -4 & 3 & $5-2 \epsilon$ \\
\hline
\end{tabular}

Consider first the game in Table 10. The respective relative payoff gains are $\eta_{a b}=$ $\frac{2(1-\epsilon)}{3}, \eta_{b a}=\frac{1+2 \epsilon}{3}, \eta_{b c}=2 \epsilon, \eta_{c b}=1-2 \epsilon, \eta_{a c}=\frac{2(1+\epsilon)}{3}, \eta_{c a}=\frac{1}{2-\epsilon}$. Clearly, no strategy is uniquely globally contagious nor uniquely path-wise contagious even for networks with contagion threshold of $\frac{1}{2}$. There are two path-wise contagious paths for networks with contagion threshold of $\frac{1}{2}: H_{\mathbf{b a}}=\{\mathbf{b} \rightarrow \mathbf{a}\}$ and $H_{\mathbf{b} \mathbf{c}}=\{\mathbf{b} \rightarrow \mathbf{c}\}$. Implying that the asymptotically stable states are $\mathbf{b}$ and $\mathbf{a}$ in such networks. 
Table 11: Strategy $a$ is globally pairwise risk-dominant and $0<\epsilon<\frac{1}{5}$.

\begin{tabular}{c|c|c|c|c|}
\multicolumn{1}{c}{$\mathrm{a}$} & $\mathrm{b}$ & $\mathrm{c}$ & $\mathrm{d}$ \\
\cline { 2 - 5 } $\mathrm{a}$ & 0 & 2 & 2 & $-1+2 \epsilon$ \\
\cline { 2 - 5 } $\mathrm{b}$ & $-2+2 \epsilon$ & $3+2 \epsilon$ & 4 & $1+3 \epsilon$ \\
$\mathrm{n}$ & -4 & 3 & $5-2 \epsilon$ & 4 \\
\hline $\mathrm{d}$ & 0 & $3+2 \epsilon$ & $5-2 \epsilon$ \\
\hline
\end{tabular}

Now, consider the game in Table 11. In addition to the above relative payoff gains, we have $\eta_{a d}=\frac{3-2 \epsilon}{4}, \eta_{d a}=\frac{5-2 \epsilon}{9-2 \epsilon}, \eta_{b d}=\frac{3}{4-2 \epsilon}, \eta_{d b}=\frac{3-3 \epsilon}{3-\epsilon}, \eta_{c d}=\frac{2}{3}$ and $\eta_{d c}=\frac{1}{3}$. The only path-wise contagious path is $H_{\mathbf{d c}}=\{\mathbf{d} \rightarrow \mathbf{c}\}$.

Before establishing what the long-run stable states are, first note that intermediate limit states ( limit sates that consist of more than one pure strategy) may or may not exist depending on the network structure. For example, for networks with contagion threshold of $\frac{1}{2}$, Alós-Ferrer and Weidenholzer [8] refer to them as $a b c$-segregated states and are of the form: . . aaaabcccbaaa... These $a b c$-segregated states arise from the fact that under cyclic interactions (that is the $\frac{1}{2}$ contagion threshold network), when play is in state a, a mutation to $d$ will induce neighbors of the mutant to play $c$. In the following period the mutant switches to playing $c$ and the neighbors of the those playing $c$ will switch to playing $b$, resulting to the limit state ...aaaabcccbaaa...

In this game, we can use the notion of global and path-wise contagion to deduce states that are asymptotically globally stable. Since $\eta_{b a}=\frac{1+2 \epsilon}{3}>\frac{1}{3}$, it follows that $a$ is not contagious relative to $b$ for all networks with contagion threshold of $\frac{1}{3}$. Leaving $\mathbf{c}$ to be the asymptotically globally stable state. For networks with contagion threshold between $\frac{1}{3}$ and $\frac{1}{2}$ strategy $a$ is contagious relative to $b$ and hence a is potentially stable in the long-run. However, due to existence of $a b c$-segregated states, a becomes as equally likely as the former. Leaving $\mathbf{c}$ to be the states with highest probability of occurrence in the long-run. Conclusively, even though $a$ is the globally pairwise risk-dominant strategy, it is not asymptotically globally stable. 


\subsection{CONCLUDING REMARKS}

This Chapter develops methods for stochastic evolutionary equilibrium selection in networks. We defined asymptotic global stability as an appropriate solution concept. We then defined two concepts that are compatible with asymptotic global stability: global and path-wise contagion. Strategies that are uniquely globally or path-wise contagious are also uniquely asymptotically globally stable. These two concepts are used to analyze how the network and payoff structures interactively influence asymptotic global convergence hence stability. We show that under local interactions, asymptotic global convergence is robust to the model of mistakes and addition of strictly dominated strategies. But perhaps most importantly, we show that for a given payoff structure and hence relative payoff gains, it is possible to design a network such that a unique strategy is asymptotically globally stable. Similarly, for a given network structure and set of strategies, one can determine the relative payoff gains that lead to a given strategy to be globally stable. The results we find have both practical and empirical implications that have been briefly discussed in the introduction.

\subsection{APPENDIX}

\subsubsection{Proof of Lemma 5}

The proof follows by considering the properties of the reduced chain $\left(\tilde{\Omega}_{,}, \tilde{P}_{N, \varepsilon}\right)$ with stationary distribution $\tilde{\pi}_{N}$. The formal definition of $\left(\tilde{\Omega}, \tilde{P}_{N, \varepsilon}\right)$ is as follows (a generalization of the collapsed Markov chain in Aldous and Fill [4, Chapter 2]):

$$
\begin{aligned}
\tilde{P}_{N, \varepsilon}(\mathbf{x}, \mathbf{y}) & =P_{N, \varepsilon}(\mathbf{x}, \mathbf{y}) \\
\tilde{P}_{N, \varepsilon}(\mathbf{y}, \tilde{\Omega}) & =\sum_{\mathbf{x} \in \tilde{\Omega}} P_{N, \varepsilon}(\mathbf{y}, \mathbf{x}), \\
\tilde{P}_{N, \varepsilon}(\tilde{\Omega}, \mathbf{y}) & =\frac{1}{\pi_{N}(\tilde{\Omega})} \sum_{\mathbf{x} \in \tilde{\Omega}} \pi_{N}(\mathbf{x}) P_{N, \varepsilon}(\mathbf{x}, \mathbf{y}), \\
\tilde{P}_{N, \varepsilon}\left(\tilde{\Omega}, \tilde{\Omega}^{\prime}\right) & =\frac{1}{\pi_{N}(\tilde{\Omega})} \sum_{\mathbf{x} \in \tilde{\Omega}} \sum_{\mathbf{y} \in \tilde{\Omega}^{\prime}} \pi_{N}(\mathbf{x}) P_{N, \varepsilon}(\mathbf{x}, \mathbf{y})
\end{aligned}
$$

The following lemma is an immediate consequence of the above definition of a collapsed Markov chain.

Lemma 6: Let $\pi_{N}$ and $\tilde{\pi}_{N}$ be the stationary distributions of $\left(\mathbf{X}, P_{N, \varepsilon}\right)$ and $\left(\tilde{\Omega}_{,} \tilde{P}_{N, \varepsilon}\right)$ respectively. Then for any $\tilde{\Omega} \in \tilde{\Omega}, \tilde{\pi}_{N}(\tilde{\Omega})=\pi_{N}(\tilde{\Omega})=\sum_{\mathbf{x} \in \tilde{\Omega}} \pi_{N}(\mathbf{x})$ 
Proof. Note that $\pi_{N}(\tilde{\Omega})=\sum_{\mathbf{x} \in \tilde{\Omega}} \pi_{N}(\mathbf{x})$. Let $\# \mathbf{X}$ and $\# \tilde{\Omega}$ be the cardinality of $\mathbf{X}$ and $\tilde{\Omega}$ respectively. Define an event matrix $\mathcal{E}$ as an $\# \mathbf{X} \times \# \tilde{\Omega}$ matrix whose entries take on a value one if a state $\mathbf{x}$ belongs to $\tilde{\Omega}$ and zero otherwise. Denote by $\mathcal{E}_{\tilde{\Omega}}$ for the $\tilde{\Omega}^{\text {th }}$ column of $\mathcal{E}$. It then follows that $\tilde{P}_{N, \mathcal{E}} \mathcal{E}=\mathcal{E} \tilde{P}_{N, \varepsilon}$, and that

$$
\pi_{N}(\tilde{\Omega})=\pi_{N} \mathcal{E}_{\tilde{\Omega}} \quad \forall \tilde{\Omega} \in \tilde{\Omega}
$$

Consequently, $\pi_{N} \mathcal{E}=\pi_{N} \tilde{P}_{N, \mathcal{E}} \mathcal{E}=\pi_{N} \mathcal{E} \tilde{P}_{N, \mathcal{E}}$. Implying that $\pi_{N} \mathcal{E}$ is the stationary distribution of $\tilde{P}_{N, \varepsilon}$, hence $\tilde{\pi}_{N}=\pi_{N} \mathcal{E}$.

Now, since the reduced chain is also irreducible just like the original chain, for any pair of subsets $\tilde{\Omega}, \tilde{\Omega}^{\prime} \in \tilde{\Omega}$ with $U_{\tilde{\Omega} \tilde{\Omega}^{\prime}}=\tilde{\Omega} \cup \tilde{\Omega}^{\prime}$ and $U_{\tilde{\Omega} \tilde{\Omega}^{\prime}}^{c}$ its complement.

$$
\begin{gathered}
\pi_{N, \varepsilon}(\tilde{\Omega}) \tilde{P}_{N, \varepsilon}(\tilde{\Omega}, \tilde{\Omega})+\pi_{N, \varepsilon}\left(\tilde{\Omega}^{\prime}\right) \tilde{P}_{N, \varepsilon}\left(\tilde{\Omega}^{\prime}, \tilde{\Omega}\right)+\pi_{N, \varepsilon}\left(U_{\tilde{\Omega} \tilde{\Omega}^{\prime}}^{c}\right) \tilde{P}_{N, \varepsilon}\left(U_{\tilde{\Omega} \tilde{\Omega}^{\prime \prime}}^{c} \tilde{\Omega}\right)=\pi_{N, \varepsilon}(\tilde{\Omega}) \\
\pi_{N, \varepsilon}(\tilde{\Omega})\left(1-\tilde{P}_{N, \varepsilon}(\tilde{\Omega}, \tilde{\Omega})\right)=\pi_{N, \varepsilon}\left(\tilde{\Omega}^{\prime}\right) \tilde{P}_{N, \varepsilon}\left(\tilde{\Omega}^{\prime}, \tilde{\Omega}\right)+\pi_{N, \varepsilon}\left(U_{\tilde{\Omega} \tilde{\Omega}^{\prime}}^{c}\right) \tilde{P}_{N, \varepsilon}\left(U_{\tilde{\Omega} \tilde{\Omega}^{\prime}}^{c}, \tilde{\Omega}\right) \\
\frac{\pi_{N, \varepsilon}\left(\tilde{\Omega}^{\prime}\right)}{\pi_{N, \varepsilon}(\tilde{\Omega})}=\frac{1-\tilde{P}_{N, \varepsilon}(\tilde{\Omega}, \tilde{\Omega})}{\tilde{P}_{N, \varepsilon}\left(\tilde{\Omega}^{\prime}, \tilde{\Omega}\right)}-\frac{\pi_{N, \varepsilon}\left(U_{\tilde{\Omega} \tilde{\Omega}^{\prime}}^{c}\right)}{\pi_{N, \varepsilon}(\tilde{\Omega})} \frac{\tilde{P}_{N, \varepsilon}\left(U_{\tilde{\Omega} \tilde{\Omega}^{\prime \prime}}^{c}, \tilde{\Omega}\right)}{\tilde{P}_{N, \varepsilon}\left(\tilde{\Omega}^{\prime}, \tilde{\Omega}\right)}
\end{gathered}
$$

By substituting for $1-\tilde{P}_{N, \varepsilon}(\tilde{\Omega}, \tilde{\Omega})=\tilde{P}_{N, \varepsilon}\left(\tilde{\Omega}, \tilde{\Omega}^{c}\right)$, we then have

$$
\frac{\pi_{N, \varepsilon}\left(\tilde{\Omega}^{\prime}\right)}{\pi_{N, \varepsilon}(\tilde{\Omega})} \leq \frac{\tilde{P}_{N, \varepsilon}\left(\tilde{\Omega}, \tilde{\Omega}^{c}\right)}{\tilde{P}_{N, \varepsilon}\left(\tilde{\Omega}^{\prime}, \tilde{\Omega}\right)}
$$

Since we are considering simultaneous revision protocol, the transition $\tilde{\Omega} \rightarrow \tilde{\Omega}^{\prime}$ can equivalently be treated as a step-by-step transition $\partial \Omega \rightarrow \Omega \rightarrow \partial \Omega^{\prime} \rightarrow \Omega^{\prime}$. Then

$$
\tilde{P}_{N, \varepsilon}\left(\tilde{\Omega}, \tilde{\Omega}^{\prime}\right) \equiv P_{N, \varepsilon}(\partial \Omega, \Omega) P_{N, \varepsilon}\left(\Omega, \partial \Omega^{\prime}\right) P_{N, \varepsilon}\left(\partial \Omega^{\prime}, \Omega^{\prime}\right)
$$

The above argument follows from the fact that under simultaneous revision protocol, at least $R\left(\Omega, \partial \Omega^{\prime}\right)$ players must simultaneous make a mistake for the transition $\Omega \rightarrow$ $\partial \Omega^{\prime}$ to occur. Meaning that the process spends most time in $\Omega$ when in the basin of attraction $\tilde{\Omega}$. Hence, the following approximation holds

$$
\tilde{P}_{N, \varepsilon}\left(\tilde{\Omega}, \tilde{\Omega}^{\prime}\right) \approx P_{N, \varepsilon}\left(\Omega, \Omega^{\prime}\right)=P_{N, \varepsilon}\left(\Omega, \partial \Omega^{\prime}\right) P_{N, \varepsilon}\left(\partial \Omega^{\prime}, \Omega^{\prime}\right)
$$

The relation (3.3.2) follows from the chain-rule argument.

\subsubsection{Proof of Proposition 5}

We begin by proving the following lemma. 
LeMMA 7: Let $L$ be the number of closed communication classes and $\mu_{j}$ the jth eigenvalue of $P_{N, \varepsilon}$. Then the contagion rate within any basin of attraction $\Omega_{l}$ has the following lower and upper bounds

$$
1-\mu_{L+1} \leq \mathcal{R}\left(\partial \Omega_{l}, \Omega_{l}\right) \leq 1-\mu_{L+L}
$$

Proof. The proof makes use of the spectral properties and near-complete decomposability of transition matrix $P_{N, \varepsilon}$. Under chain $(\mathbf{X}, P)$, the transition matrix is completely decomposable into the form

$$
P_{N}=\left(\begin{array}{ccccc}
M_{1}^{*} & & & & \\
& \ddots & & & \\
& & M_{l}^{*} & & \\
& & & \ddots & \\
& & & & M_{L}^{*}
\end{array}\right)
$$

where $M_{l}^{*}$ for $l=1, \cdots, L$ is a block matrix describing the transitions within each basin of attraction under $\left(\mathbf{X}, P_{N}\right)$. The rest of the undisplayed elements are zeros and $L$ is the number of closed communication classes. All leading eigenvalues of the block matrices are ones. The transition matrix $P_{N, \varepsilon}$ on the other hand is nearcompletely decomposable into $L$ "loosely" connected block matrices that we denote by $M_{l}$ for $l=1, \cdots, L$. $P_{N, \varepsilon}=P_{N}+\epsilon P_{N}^{*}$, where $\epsilon$ is a small real number and $P_{N}^{*}$ is an arbitrary $\# \mathbf{X}$ by $\# \mathbf{X}$ matrix. A more detailed exposition on the notion of near-complete decomposability can be found in Simon and Ando [106]. For $\epsilon$ small enough, the leading eigenvalues of the diagonal block matrices of $P_{N, \varepsilon}$ are close to one.

Let $\mu_{i_{l}}$ denote the $i$ th eigenvalue of the $l$ th diagonal block matrix, such that

$$
\left(\mu_{1_{1}}, \mu_{1_{2}}, \cdots, \mu_{1_{L}}\right)
$$

are the largest eigenvalues in blocks 1 to $L$, and $\left(\mu_{2_{1}}, \mu_{2_{2}}, \cdots, \mu_{2_{L}}\right)$ are the respective second largest eigenvalues. Index by $n_{l}$ as the number of columns in diagonal block $l$ such that the eigenvalue spectrum $\rho\left(P_{N, \varepsilon}\right)$ of $P_{N, \varepsilon}$ can be written as

$$
\rho\left(P_{N, \varepsilon}\right)=\left(\mu_{1_{1}}, \mu_{2_{1}}, \cdots, \mu_{n_{11}}, \cdots, \mu_{1_{2}}, \cdots, \mu_{1_{l}}, \cdots, \mu_{n_{l 1}}, \cdots, \mu_{1_{L}}, \cdots, \mu_{n_{L L}}\right) .
$$

The spectral decomposition of $\left(\mathbf{X}, P_{N, \varepsilon}\right)$ is then given by

$$
\begin{aligned}
\mathbf{q}_{0} P_{N, \varepsilon}^{t} & =\mathbf{q}_{0} \mathbf{r}_{1_{1}} \mathbf{z}_{1_{1}}^{T}+\sum_{j=2}^{n_{1}} \mu_{j_{1}}^{t} \mathbf{q}_{0} \mathbf{r}_{j_{1}} \mathbf{z}_{j_{1}}^{T}+\mu_{1_{2}}^{t} \mathbf{q}_{0} \mathbf{r}_{1_{2}} \mathbf{z}_{1_{2}}^{T}+\sum_{j=2}^{n_{2}} \mu_{j_{2}}^{t} \mathbf{q}_{0} \mathbf{r}_{j_{2}} \mathbf{z}_{j_{2}}^{T} \\
& +\cdots+\mu_{1_{L}}^{t} \mathbf{q}_{0} \mathbf{r}_{1_{L}} \mathbf{z}_{1_{L}}^{T}+\sum_{j=2}^{n_{L}} \mu_{j_{L}}^{t} \mathbf{q}_{0} \mathbf{r}_{j_{L}} \mathbf{z}_{j_{L}}^{T}
\end{aligned}
$$

where $\mathbf{z}^{T}$ is the transpose of $\mathbf{z}$, and $\mathbf{r}_{j_{l}}$ and $\mathbf{z}_{j_{l}}$ are the right and left eigenvectors of $\mu_{j_{l}}$. 
Let $\mathbf{x}_{l}$ be the initial state of $\left(\mathbf{X}, P_{N, \varepsilon}\right)$ in $\tilde{\Omega}_{l}$ and $\mathbf{q}_{\tilde{\Omega}_{l}}$ be the \#X-dimensional vector of zeros except a one at the point corresponding to the state $\mathbf{x}_{l}$. Let $t_{l}$ be the period at which $\left(\mathbf{X}, P_{N, \varepsilon}\right)$ is in the state $\mathbf{x}_{l}$ and $T_{\tilde{\Omega}_{l}}$ the period at which it exits $\tilde{\Omega}_{l}$ (or equivalently the period at which it attains the quasi-stationary distribution $\left.\pi_{N, \varepsilon}\left(\tilde{\Omega}_{l}\right)\right)$. Then for $t_{l} \leq t \leq T_{\tilde{\Omega}_{l}}$ and all $l=1, \cdots, L$,

$$
\begin{aligned}
& \mathbf{q}_{t}=\mu_{1_{l}}^{t} \mathbf{q}_{\tilde{\Omega}_{l}} \mathbf{r}_{1_{l}} \mathbf{z}_{1_{l}}^{T}+\sum_{j=2}^{n_{l}} \mu_{j_{l}}^{t} \mathbf{q}_{\tilde{\Omega}_{l}} \mathbf{r}_{j_{l}} \mathbf{z}_{j_{l}}^{T} \\
& \pi_{N, \varepsilon}\left(\tilde{\Omega}_{l}\right)=\lim _{t \rightarrow T_{\tilde{\Omega}_{l}}} \mathbf{q}_{t}=\mu_{1_{l}}^{T_{\tilde{\Omega}_{l}}} \mathbf{q}_{\tilde{\Omega}_{l}} \mathbf{r}_{1_{l}} \mathbf{z}_{1_{l}}^{T} \approx \mu_{1_{l}}^{t} \mathbf{q}_{\tilde{\Omega}_{l}} \mathbf{r}_{1_{l}} \mathbf{z}_{1_{l}}^{T}
\end{aligned}
$$

where the approximation holds from the fact that $\mu_{1_{l}}$ is close to one for all $l$. It then follows that

$\limsup _{t \rightarrow T_{\tilde{\Omega}_{l}}}\left\|P_{N, \varepsilon}^{t} \mathbf{q}_{\tilde{\Omega}_{l}}-\left.\pi_{N, \varepsilon}\left(\tilde{\Omega}_{l}\right)\right|^{\frac{1}{t}}=\left|\mu_{2_{l}}\right| \limsup _{t \rightarrow T_{\tilde{\Omega}_{l}}}\right\| \mathbf{q}_{\tilde{\Omega}_{l}} \mathbf{r}_{2_{l}} \mathbf{z}_{2_{l}}^{T}+\sum_{j=3}^{n_{l}}\left(\frac{\mu_{j_{l}}}{\mu_{2_{l}}}\right)^{t} \mathbf{q}_{\tilde{\Omega}_{l}} \mathbf{r}_{j_{l}} \mathbf{z}_{j_{l}}^{T}||^{\frac{1}{t}}=\left|\mu_{2_{l}}\right|$

Implying that $\mathcal{R}\left(\partial \Omega_{l}, \Omega_{l}\right)=\left|\mu_{2_{l}}\right|$ Since $\mu_{j}$ 's are arranged in ascending order we then have that $\max _{l} \mu_{2_{l}}=\left|\mu_{L+1}\right|$ and $\min _{l} \mu_{2_{l}}=\left|\mu_{L+L}\right|$. For any $\Omega_{l}$,

$$
1-\mu_{L+1} \leq \mathcal{R}\left(\partial \Omega_{l}, \Omega_{l}\right) \leq 1-\mu_{L+L}
$$

To prove the second part of the theorem, we consider the linearization of $P_{N, \varepsilon}$ of the form

$$
\mathbf{q}_{t} \Psi=\mathbf{q}_{0} P_{N, \varepsilon}^{t} \Psi=\mathbf{q}_{0} \Psi \Pi_{N, \varepsilon}^{t}
$$

where $\Psi$ is the event matrix derived by stacking into rows all possible realizations of states of $\left(\mathbf{X}, P_{N, \varepsilon}\right)$ written in the basis vector form. The choice basis vector for each player $i \in N$ is a vector of zeros except a one in a position corresponding to the action $i$ is playing. For example for a binary action set $X=\{A, B\}$, a vector $(1,0)$ implies that $i$ is playing action $A$ and $(0,1)$ implies that $i$ is playing action $B$. In the case of two players and binary action set, there are four possible realization such that

$$
\Psi=\left(\begin{array}{llll}
1 & 0 & 1 & 0 \\
1 & 0 & 0 & 1 \\
0 & 1 & 1 & 0 \\
0 & 1 & 0 & 1
\end{array}\right)
$$

where the first row corresponds to the state in which both players play action $A$, and so forth. Then $\Pi_{N, \varepsilon}$ is an $N m \times N m$ matrix defined by $\Pi_{N, \varepsilon}=\mathscr{A}^{T} \otimes \Sigma_{N, \varepsilon}$, where $\otimes$ is a Kronecker product, $\mathscr{A}^{T}$ is the transpose of the normalized adjacency matrix $\mathscr{A}$ 
and $\Sigma_{N, \varepsilon}$ is the action-transition matrix defined in (3.3.7). A detailed exposition on the validity of (3.8.3) can be found in Asavathiratham [11, Chapter 5]. The following lemma follows directly from (3.8.3) and the definition of $\Pi_{N, \varepsilon}$ above.

Lemma 8: Let $\rho\left(\Pi_{N, \varepsilon}\right)=\tilde{\mu}_{1}, \cdots, \tilde{\mu}_{n m}, \rho(\mathscr{A})=\left(\lambda_{1}, \cdots, \lambda_{N}\right)$ and $\rho\left(\Sigma_{N, \varepsilon}\right)=\left(\vartheta_{1}, \cdots, \vartheta_{m}\right)$ denote the eigenvalue spectra of $\Pi_{N, \varepsilon}, \mathscr{A}$ and $\Sigma_{N, \varepsilon}$ respectively.

(a) If $\mu_{1}$ and $\tilde{\mu}_{1}$ are the unique largest eigenvalues of $P_{N, \varepsilon}$ and $\Pi_{N, \varepsilon}$ respectively, then $\mu_{1}=\tilde{\mu}_{1}=1$.

(b) $\rho\left(\Pi_{N, \varepsilon}\right)=\left(\vartheta_{i} \lambda_{j}\right) \forall \vartheta_{i} \in \rho\left(\Sigma_{N, \varepsilon}\right), \lambda_{j} \in \rho(\mathscr{A})$.

Proof. Multiplying (3.8.3) by the right eigenvector $\mathbf{r}_{i}$ of $P_{N, \varepsilon}$, we have $P_{N, \varepsilon} \Psi \mathbf{r}_{1}=$ $\Psi \Pi_{N, \varepsilon} \mathbf{r}_{1}$. Since $P_{N, \varepsilon}$ is a stochastic matrix, $\mu_{1}=1$, which implies that $P_{N, \varepsilon} \Psi \mathbf{r}=\Psi \mathbf{r}_{1}$, which is true if and only if $\Pi_{N, \varepsilon} \mathbf{r}_{1}=\mathbf{r}_{1}$. That is $\tilde{\mu}_{1}=\mu_{1}=1$. For the proof of Lemma 8 (b) see Horn and Johnson [52, page 245, Theorem 4.2.12].

For sufficiently small noise, $\left|\mu_{L+1}\right|=\left|\tilde{\mu}_{L+1}\right|=\lambda_{2} \vartheta_{1}=\lambda_{2}$ and $\left|\mu_{L+L}\right|=\left|\tilde{\mu}_{L+L}\right|=$ $\lambda_{2} \vartheta_{m}$. This completes the proof.

\subsubsection{Proofs for Example 6}

Let $G=(\mathcal{N}, E)$ be a graph or network of $N$ vertices. Denote by $\mathcal{S}$ a subset of $\mathcal{N}$ and $S$ its cardinality. Let $e(S, N-S)$ be the number of interactions (for a weighted network graph its is the sum of weighted interactions) between $\mathcal{S}$ and its complement $\mathcal{N}-\mathcal{S}$. Also let $d(\mathcal{S})$ denote the total degree of $\mathcal{S}$. Then the conductance of $G$

$$
\phi(G)=\min _{\mathcal{S}, S \leq \frac{N}{2}} \frac{e(S, N-S)}{d(\mathcal{S})}
$$

For regular network graphs (in which all vertices have the same degree), it is shown by [6] that

$$
\lambda_{2}(G) \leq 1-\frac{\phi(G)^{2}}{2}
$$

For a complete graph, since every vertex is connected to every other vertex, we have that every vertex in $\mathcal{S}$ is connected to all other vertices in $\mathcal{N}-\mathcal{S}$. This implies that $e(S, N-S)=S \times \#(\mathcal{N}-\mathcal{S})=S \times(N-S)$, and $d(\mathcal{S})=N \times S$ such that

$$
\phi\left(G_{c o m}\right)=\min _{\mathcal{S}, S \leq \frac{N}{2}} \frac{S \times(N-S)}{N \times S} \geq \frac{1}{2},
$$

where the last inequality follows from the fact that $S \leq \frac{N}{2}$. We thus have that

$$
\lambda_{2}\left(G_{\text {com }}\right) \leq \frac{7}{8}
$$


In the case of a $1-D$ cyclic network, $e(S, N-S)=2$, and $d(\mathcal{S})=2 \times S$ such that

$$
\phi\left(G_{c y c}\right)=\min _{\mathcal{S}, S \leq \frac{N}{2}} \frac{2}{2 \times S} \geq \frac{2}{N} .
$$

Hence $\lambda_{2}\left(G_{c y c}\right) \leq \frac{N^{2}-2}{N^{2}}$.

$2 D$ network: Let the composition of $\mathcal{S}$ be chosen in such a way that the peripheral vertices (vertices at the perimeter or boundary of $\mathcal{S}$ ) contain approximately one edge each connecting it to the set $\mathcal{N}-\mathcal{S}$. Since it is a 2-dimensional structure there should be approximately $\sqrt{S}$ vertices forming such a boundary. This implies that $e(S, N-$ $S) \approx \sqrt{S}$, and $d(\mathcal{S})=4 \times S$ such that

$$
\phi\left(G_{2 D}\right)=\min _{\mathcal{S}, S \leq \frac{N^{2}}{2}} \frac{\sqrt{S}}{4 \times S} \geq \frac{\sqrt{2}}{4 N}
$$

where the last inequality follows from the fact that $\sqrt{S} \leq \sqrt{\frac{N^{2}}{2}}$. It follows that

$$
\lambda_{2}\left(G_{2 D}\right) \leq \frac{16 N^{2}-1}{16 N^{2}} .
$$

Random $d$-regular network: Since for each vertex the vertices to which it is connected are chosen at random, and that the maximum size of $\mathcal{S}$ is $\frac{N}{2}$, then a typical vertex in $\mathcal{S}$ is connected to approximately $\frac{d \times(N-S)}{N}$ other vertices in $\mathcal{N}-\mathcal{S}$ such that $e(S, N-S) \approx \frac{d \times S(N-S)}{N}$. We thus have

$$
\begin{gathered}
\phi\left(G_{d-r}\right)=\min _{\mathcal{S}, S \leq \frac{N}{2}} \frac{\frac{d \times S(N-S)}{N}}{d \times S} \geq 1 . \\
\lambda_{2}\left(G_{d-r}\right) \leq \frac{7}{8}
\end{gathered}
$$

For Newman's small world networks see Durrett [36].

\subsubsection{Proof of Proposition 7}

(i) From Lemma 5 and Proposition 4 it directly follows that

$$
\frac{\pi_{N, \varepsilon}\left(\tilde{\Omega}^{\prime}\right)}{\pi_{N, \varepsilon}(\tilde{\Omega})} \leq \frac{\max _{\Omega^{\prime \prime}} P_{N, \varepsilon}\left(\Omega, \partial \Omega^{\prime \prime}\right)}{P_{N, \varepsilon}\left(\Omega^{\prime}, \partial \Omega\right)} \leq K \exp \left\{N\left(\psi^{N}\left(\Omega^{\prime}, \Omega\right)-\psi_{\min }^{N}(\Omega)\right)\right\}
$$

Implying that the subset of states $\Omega^{*}$ containing the asymptotically globally stable state is that for which

$$
\lim _{N \rightarrow \infty} \frac{1}{N} \psi^{N}\left(\Omega^{\prime}, \Omega^{*}\right)<\lim _{N \rightarrow \infty} \frac{1}{N} \psi_{\min }^{N}\left(\Omega^{*}\right)
$$


for all $\Omega^{\prime} \neq \Omega^{*}$. Or equivalently, $\psi^{L}\left(\Omega^{*}\right)<0$.

(ii) For every closed communication class say $\Omega_{J}$, let $H_{\Omega_{j} \Omega_{J}}$ be the typical directed path from any other closed communication class $\Omega_{j}$ to $\Omega_{J}$. Then from (3.3.2)

$$
\begin{aligned}
\frac{\pi_{N, \varepsilon}\left(\tilde{\Omega}^{1}\right)}{\pi_{N, \varepsilon}\left(\tilde{\Omega}^{J}\right)} & =\frac{\pi_{N, \varepsilon}\left(\tilde{\Omega}^{1}\right)}{\pi_{N, \varepsilon}\left(\tilde{\Omega}^{2}\right)} \cdots \frac{\pi_{N, \varepsilon}\left(\tilde{\Omega}^{J-1}\right)}{\pi_{N, \varepsilon}\left(\tilde{\Omega}^{J}\right)} \leq \frac{P_{N, \varepsilon}\left(\Omega^{2}, \Omega^{2^{c}}\right)}{P_{N, \varepsilon}\left(\Omega^{1}, \Omega^{2}\right)} \cdots \frac{P_{N, \varepsilon}\left(\Omega_{,}^{J} \Omega^{J^{c}}\right)}{P_{N, \varepsilon}\left(\Omega^{J-1}, \Omega^{J}\right)} \\
& \leq K_{P} \exp \left\{N\left(\sum_{j=1}^{J-1}\left(\psi^{N}\left(\Omega_{j}, \Omega_{j+1}\right)-\psi_{\min }^{N}\left(\Omega_{j+1}\right)\right)\right)\right\}
\end{aligned}
$$

Where $\xi^{N}\left(H_{\Omega_{1} \Omega_{J}}\right)=\sum_{j=1}^{J-1}\left(\psi^{N}\left(\Omega_{j}, \Omega_{j+1}\right)-\psi_{\min }^{N}\left(\Omega_{j+1}\right)\right)$. Now, consider the maximum probability paths (or equivalently the minimum cost paths) from any closed communication class $\Omega_{j}$ to another $\Omega_{J}$. That is arg $\max _{H_{\Omega_{j} \Omega_{J}} \in \mathscr{H}_{\Omega_{j} \Omega_{J}}} \xi^{N}\left(H_{\Omega_{j} \Omega_{J}}\right)$.

Then the asymptotically globally stable set $\Omega^{*}$, for which $\lim _{N \rightarrow \infty} \frac{1}{N} \pi_{N, \varepsilon}\left(\Omega^{*}\right)>$ $\lim _{N \rightarrow \infty} \frac{1}{N} \pi_{N, \varepsilon}(\Omega) \forall \Omega \neq \Omega^{*}$, is that in which $\xi^{L}\left(H_{\Omega \Omega^{*}}\right)<0$. Or equivalently

$$
\xi^{L}\left(\Omega^{*}\right)=\max _{\Omega \neq \Omega^{*}} \min _{\Omega \Omega^{*} \in \mathscr{H}_{\Omega \Omega^{*}}} \xi\left(H_{\Omega \Omega^{*}}\right)<0
$$

\subsubsection{Proof of Theorem 2}

(i) From the expression $\psi^{N}\left(\Omega, \Omega^{\prime}\right)=N\left(c\left(\Omega, \partial \Omega^{\prime}\right)+F\left(r\left(\Omega, \partial \Omega^{\prime}\right)\right)\right)-\gamma\left(\Omega^{\prime}\right)$, if $\lim _{N \rightarrow \infty} \frac{1}{N} \ln \left(1-\lambda_{2}^{N}(G)\right)=0$ then for all pairs $\left(\Omega, \Omega^{\prime}\right)$ of $\Omega$

$$
\lim _{N \rightarrow \infty} \frac{1}{N} \psi^{N}\left(\Omega, \Omega^{\prime}\right)=\lim _{N \rightarrow \infty}\left\{c\left(\Omega, \partial \Omega^{\prime}\right)+F\left(r\left(\Omega, \partial \Omega^{\prime}\right)\right)\right\}
$$

(ii) If for any $\Omega_{j} \neq \Omega^{*}, r\left(\Omega_{j}, \partial \Omega^{*}\right)=\mathcal{O}\left(N^{-1}\right)$ then (3.8.15) becomes

$$
\lim _{N \rightarrow \infty} \frac{1}{N} \psi^{N}\left(\Omega, \Omega^{\prime}\right)=\lim _{N \rightarrow \infty} c\left(\Omega, \partial \Omega^{\prime}\right)=-\lim _{N \rightarrow \infty} r\left(\Omega, \partial \Omega^{\prime}\right) \ln \mathbb{P}_{A}\left(x_{\Omega^{\prime}}\right)=0
$$

where the first equality follows from the fact that $F(0)=F(1)=0$. Note that $r\left(\Omega_{j}, \partial \Omega^{*}\right)=\mathcal{O}\left(N^{-1}\right)$ in turn implies that either $r\left(\Omega^{*}, \partial \Omega_{j}\right)=\mathcal{O}(N)$ or $r\left(\Omega^{*}, \partial \Omega_{j}\right)=\mathcal{O}(1)$. Either way,

$$
\lim _{N \rightarrow \infty} \frac{1}{N} \psi^{N}\left(\Omega^{*}, \partial \Omega_{j}\right)=\lim _{N \rightarrow \infty} c\left(\Omega^{*}, \partial \Omega_{j}\right)>0
$$

Hence, $\xi^{L}\left(\Omega^{*}\right)=\lim _{N \rightarrow \infty} \frac{1}{N}\left\{\psi_{\max }^{N}\left(\Omega^{*}\right)-\psi_{\min }^{N}\left(\Omega^{*}\right)\right\}<0$. 
(iii) For any $\Omega_{j} \neq \Omega^{*}$, if each ordered pair $\left(\Omega_{j_{1}}, \Omega_{j_{2}}\right) \in H_{\Omega_{j} \Omega^{*}}, r\left(\Omega_{j_{1}}, \Omega_{j_{2}}\right)=$ $\mathcal{O}\left(N^{-1}\right)$ then

$$
\begin{aligned}
\lim _{N \rightarrow \infty} \frac{1}{N} \xi^{N}\left(H_{\Omega_{j} \Omega^{*}}\right) & =\lim _{N \rightarrow \infty} \frac{1}{N}\left\{\sum_{j_{i}=j_{1}}^{j_{I}-1}\left(\psi^{N}\left(\Omega_{j_{i}} \Omega_{j_{i}+1}\right)-\psi_{\min }^{N}\left(\Omega_{j_{i}+1}\right)\right)\right\} \\
& =-\lim _{N \rightarrow \infty} \frac{1}{N} \sum_{j_{i}=j_{1}}^{j_{I}-1} \psi_{\min }^{N}\left(\Omega_{j_{i}+1}\right)
\end{aligned}
$$

where $\Omega_{j_{I}}=\Omega^{*}$ and $\Omega_{j_{1}}=\Omega_{j}$. It then follows that if there exists at least one $\Omega_{j_{i}}$ in the path $H_{\Omega_{j} \Omega^{*}}$ for which $\psi_{\min }^{N}\left(\Omega_{j_{i}}\right)=\mathcal{O}(N), \xi^{L}\left(\Omega^{*}\right)=\lim _{N \rightarrow \infty} \frac{1}{N} \xi^{N}\left(H_{\Omega_{j} \Omega^{*}}\right)<$ 0 



\section{4}

\section{CONVERGENCE RATES FOR STOCHASTIC EVOLUTION IN NETWORKS}

AвSTRACT: Stochastic evolutionary models are credited for their ability to derive sharp predictions about equilibrium selection when multiplicity exists. A major criticisms of these models concerns the convergence rates to long-run stable sets and to stationarity. In particular, there are cases in which the expected waiting times increase exponentially with the population size. Under local interactions however, the expected waiting times are shown to be finitely bounded even when the population size is arbitrarily large. This Chapter provides a detailed characterization of convergence rates in networks using two measures; expected waiting time and convergence time as measures for the transition times between subsets of states and to stationarity or selection respectively. We provide tighter bounds for each for positive rather than limit noise levels. Three factors are key in influencing the convergence rates, the payoff gains, network topology and noise level. The network topology and payoff gains interactively determine whether or not a strategy is globally contagious and the convergence rates are a function of this property. When global contagion is feasible, the expected waiting time from any other subset of states to the long-run stable set is shorter for highly than sparsely connected networks. The reverse is true for convergence time. Networks for which global contagion is infeasible lead to shorter convergence time.

\subsection{INTRODUCTION}

As discussed in Chapters 2 and 3, one of the major discussions in stochastic evolutionary literature concerns the rate at which equilibrium selection occurs. The computation of stochastically stable sets involves taking the limits of noise. But as pointed out by Ellison [37] and Sandholm [98], assuming vanishing noise levels also implies that the convergence time to the long-run stationary distribution (hence se- 
lection) becomes exceptionally long. More specifically, once the process gets trapped in one of the quasi-stable states (possibly a socially undesirable one), the waiting time to exit its basin of attraction becomes arbitrarily large for vanishing noise. There are however cases in which the waiting times are shown to be finite and even sometimes to be independent of the population size. This is particularly the case when agents are matched to play with only a subset of others in the population. Robson and Vega-Redondo [94] show that the convergence rates are high when players are matched in a round robin tournament mechanism, where each player confronts each other player exactly once. Ellison [37], Sandholm [99], Montanari and Saberi [77] show that under local interaction (networks), equilibrium selection is fast. Recent work by Kreindler and Young [63] demonstrates that even under global interactions, it is possible to derive conditions on the payoff for the given noise levels and vice versa for which selection is fast (occurs in a finite time).

This Chapter provides a detailed analysis of convergence rates for general stochastic evolutionary game dynamics in networks. The two convergence rate measures that we characterize explicitly are the expected waiting time and convergence or selection time. The expected waiting time measures how long it takes the learning process to exit a given domain or subset of states, and in particular we will be talking about exiting a given (or subset of) basin of attraction. It is therefore a measure of mediumrun dynamics of the process. The convergence time measures how fast the process attains its long-run invariant distribution, or equivalently the time scales for which equilibrium selections results should be considered relevant.

Our characterization of expected waiting times utilizes methods for the concept of hitting time. Hitting time is a classical concept in the study of finite Markov chains and processes. The decay rates for hitting times in the case of Markov chains with rare transitions resulting from small perturbations (as is the case in this Chapter) is characterized in detail by Freidlin and Wentzell [42] and Catoni [26] using techniques from large deviations theory. Beggs [14] shows how the results and concepts developed in Freidlin and Wentzell [42] and Catoni [26] can be used to characterize expected waiting times and equilibrium selection in stochastic evolutionary models. The analysis in Beggs [14] improves on the earlier results by Ellison [37] on expected waiting times. Though these papers provide good insights on the nature of hitting times in stochastic evolutionary processes, their analysis focuses on generic characterizations. Here, we focus on expected waiting times for stochastic evolution in networks. To be able to fully characterize the effects of various network topologies, we adopt a simpler model of mistakes, in which the probability of making a mistake $\mathscr{P}=\frac{1}{m} \exp (-\beta)$, is identical for all players, strategies and states. The parameter $m$ 
is the size of the action set and $\beta$ is the parameter of noise, such that the larger $\beta$ the smaller the probability of mistakes. For $\beta$ equal to infinity, we have the case of best-response dynamics.

The characterization of convergence time that we provide utilizes methods from the concept of mixing time. Mixing time is also a traditional concept in finite Markov chains and processes. It is a measure of the time it takes the chain to attain its invariant distribution. Since long-run or stochastically stable sets solely depend on the properties of the invariant distribution, mixing time can thus be employed to characterize the time to selection. The structural bounds for mixing time of finite Markov chains have been established in the literature (e.g Diaconis and Stroock [33] and Sinclair [107]). Its lower and upper bounds are a function of the second largest eigenvalue of the associated transition matrix. Diaconis and Stroock [33] and Sinclair [107] have established geometric bounds for the second eigenvalue of the Markov chain transition matrices. These measure is however computationally demanding for large state space chains. They are also less intuitive in the case of stochastic evolution in which the interest is to understand how the underlying game, learning rules and network topology affect the convergence time. Here, we provide bounds for convergence time in terms of the readily computable and fairly understood concept in the literature of stochastic evolution; the maximum expected waiting time. The properties we derive for expected waiting time therefore extend directly to the characterization of convergence time.

By explicitly characterizing bounds for convergence rates, we are able to derive deeper insights into the role of topologies in stochastic evolution in networks. We establish convergence rates results for families of deterministic and random networks. We find the key factor of the evolutionary process to be whether or not a strategy is globally contagious. For a given network topology, an action is said to be globally contagious if its payoff gain relative to all other actions is such that it requires only a small fraction of initial adopters to trigger a global cascade to the whole population. Globally contagious actions are then those with maximum payoff gains and thus form the long-run stable states. In $2 \times 2$ matrix games for example, if global contagion is feasible then the risk-dominant action is the globally contagious one. For given payoff gains, the more sparsely connected a network is the more likely that an action with the highest pain off gain is globally contagious. For a given network topology and payoff gains, if global contagion is not feasible then we have the case of step-bystep evolution.

When global contagion is feasible, then for various families of networks the expected waiting times from any other closed communication class to the long-run 
stable closed communication class are independent of the population size. This result is not particularly surprising as similar findings were obtained by Ellison [37] for $k$-nearest neighbor interactions. The main difference with Ellison [37] however is that we provide tighter bounds for expected waiting times independently of the assumption of vanishing mutation rates. Secondly, we establish this result for a wider range of networks beyond the $k$-nearest neighbor interactions. More generally, for networks of the same family such as the $n$-dimensions nearest neighbor interactions or $n$-dimensions $l$-max distance interactions, whenever global contagion is feasible the expected waiting time for highly connected network is shorter than for sparsely connected networks. This result is not true otherwise. It is also infeasible to derive such conclusions from analysis in the derivation of expected waiting times that heavily relies on the assumption of vanishing noise.

In the case of convergence times, whenever global contagion is feasible, sparsely connected networks tend to have longer convergence time than highly connected networks. In fact, under these conditions the convergence time for global interactions is shorter than for local interactions. This is not necessarily true whenever global contagion is not feasible. The underlying reason for this finding is that convergence time is an increasing function of maximum expected waiting time. The maximum expected waiting time corresponds to the expected waiting time from the long-run stable communication class to the least stable communication class. The maximum expected waiting time for local interactions in which global contagion is feasible is generally greater than for global interactions.

Whenever global contagion is not feasible, the evolutionary process exhibits a relatively different behavior hence convergence rates as compared to the case in which it is feasible. Infeasibility of global contagion implies that there exists intermediate close communication classes induced by the network topology. These intermediate closed communication classes correspond to states in which at least two cohesive or close-knit subgroups of players play different actions. ${ }^{1}$ Lee et al. [66], Morris [78], Young [116] and Montanari and Saberi [77] show that the expected waiting times to the long-run stable closed communication class is finite. An evolutionary process with such intermediate closed communication classes also relates to the notion of step-by-step evolution according to Ellison [38], in which it is demonstrated that under step-by-step evolution the selection process is relatively fast. Here, we formalize these notions for evolutionary processes in networks using bounds we derive for expected waiting times and convergence times. Indeed, we find that the conver-

1 Cohesiveness and close-knittedness are definitions according to Morris [78] and Young [116] 
gence time (time to selection) is shorter when the network topology and payoff gains collectively induce step-by-step evolution than even the case of global interactions.

Finally, this Chapter is also related to others in the literature that characterize evolutionary processes in random networks using mean field best-response dynamics (e.g. Alós-Ferrer and Weidenholzer [9], Jackson and Yariv [55], Lelarge [68], Sandholm [99] and Kreindler and Young [63, 64]). Most of these literature, with the exception of Kreindler and Young [63,64], focuses on long-run stability rather than convergence rates as we do here. Kreindler and Young [63, 64] derive upper bounds for expected waiting times to long-run stable states and show that they are independent of the population size as long as the payoff gain is sufficiently large and the noise level is not too low. Though the analysis we present derives some results that are similar qualitatively, there are several differences in both methodology and quantitative results. Kreindler and Young $[63,64]$ focus on sequential dynamics rather than simultaneous as we do, which implies differences in analytical methods and in turn quantitative results. For example, we find the structure of the network to be relevant in determining convergence rates, unlike in Kreindler and Young $[63,64]$ in which it does not. Moreover, our analysis extends to $m \times m$ matrix games and includes convergence times.

The remainder of the Chapter is organized as follows. In section 5.2 we introduce the general framework of stochastic evolutionary dynamics in networks. Section 4.3 introduces expected waiting times, providing its general treatment and computation. Similarly, Section 4.4 introduces convergence time. Sections 4.5 and 4.6 focus on the characterizations of convergence rates for families of deterministic and random networks when global contagion is feasible. Section 4.7 provides a characterization of the case in which global contagion is not feasible. Section 4.8 concludes the study.

\subsection{THE MODEL}

We consider a general class of $N$-persons $m \times m$-matrix repeated games. A set of agents $\mathcal{N}=\{1, \cdots, i, \cdots, N\}$ are matched repeatedly to play a normal form game $\Gamma$. The matching rule determines whether agents interact with the entire population (global) or a subset of others (social network). This in turn also determines the information set of players, whether they observe the entire distribution of strategies in the population or in their local neighborhood. In the case in which agents are matched with a subset of others, the network of interactions is modeled in graph theoretic manner. Let $G(\mathcal{N}, E)$ be a graph with $\mathcal{N}$ vertices representing the number of agents and $E$ edges linking different pairs of agents, such that a graph $g_{i j}$ defines 
the connection between $i$ and $j$. If $g_{i j}=1$ then a directed link exists from $i$ to $j$, and zero implies otherwise. $G(\mathcal{N}, E)$ is thus a directed network describing the relationship of any one agent with every other agent in the population. The adjacency matrix $\mathscr{A}$ of $G(\mathcal{N}, E)$ is defined as an $N \times N$ matrix with entries being the elements of $g_{i j}$. The neighborhood of agent $i, \mathcal{N}_{i}$, is defined as $\mathcal{N}_{i}=\left\{j \in \mathcal{N} \mid g_{i j}=1\right\}$, and gives the set of players to which $i$ is linked to. The cardinality $\# \mathcal{N}_{i}=k_{i}$, is the degree of $i$.

The strategy set $X=\{a, b, c, \cdots\}$ is finite and assumed to be identical for all agents and has cardinality $m$. We denote the action chosen by each $i \in \mathcal{N}$ at period $t$ by $x_{i, t}$ and $\mathbf{x}_{t}=\left(x_{1, t}, \cdots, x_{n, t}\right)$ is the strategy profile at $t$. Each $\mathbf{x}_{t} \in \mathbf{X}=X^{N}$ will also be referred to as the population state or simply the state of the learning process at $t$.

For each game $\Gamma$, let $U \in \mathbb{R}^{m \times m}$ be the associated payoff matrix such that $U(a, b)$ is the base payoff to an agent playing $a$ when his opponent plays $b$. A typical example is the pure coordination game in Table 12. Given the information set of agent $i$, that is the observed distribution of strategies $\mathbf{x}$ either of the entire population or of $i^{\prime} \mathrm{s}$ neighborhood, the expected payoff to $i$ from playing action $a$ when his opponents profile is $\mathbf{x}_{-i}$ is then

$$
U_{i}\left(a, \mathbf{x}_{-i}\right)=\sum_{j \in \mathcal{N}_{i}} J_{i j} U\left(a, x^{j}\right),
$$

where $x^{j}$ is the $j^{\text {th }}$ coordinate of $\mathbf{x}$. The parameter $J_{i j}$ takes on values in the closed interval $[0,1]$. For example if agents consider average payoff over their neighbors' strategies, then $J_{i j}=\frac{1}{k_{i}}$ for all $j \in \mathcal{N}_{i}$.

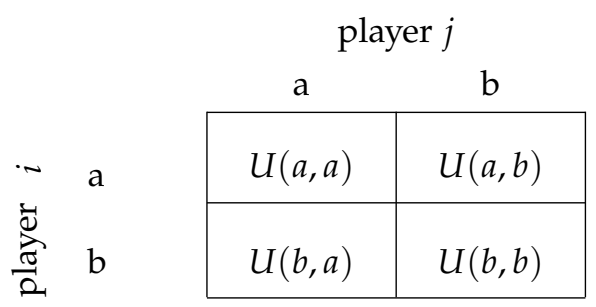

Table 12: Payoff structure for the symmetric pure coordination game. The payoffs are for the row-player

\subsubsection{Dynamics and learning scheme}

There are two main components that completely describe the learning process; the revision protocol and choice probabilities. Here, we consider the simultaneous revision protocol. When a player receives a revision opportunity, he evaluates the current 
expected payoff to each of the available pure strategies. A player's information set is the distribution of strategies of a subset of other players in the neighborhood. Each player's objective is to select strategies that he evaluates as best, this assessment is however subject to random shocks. We adopt a relatively simpler model of mistakes (hence choice probabilities) to be able to fully capture the effect of the topology of networks on convergence rates. We assume the probability of making a mistake to be $\mathscr{P}=\frac{1}{m} \exp (-\beta)$, identical for all players, strategies and states. The parameter $m$ is the size of the action set and $\beta$ is the parameter of noise, such that the larger $\beta$ the smaller the probability of mistakes. For $\beta$ equal to infinity, we have the case of best-response dynamics. The choice probabilities are thus of the form,

$$
\mathbb{P}_{i}(a, \mathbf{x})=(1-\varepsilon) B R_{i}(a, \mathbf{x})+\frac{1}{m} \varepsilon
$$

where $\varepsilon=\exp (-\beta)$ and $B R_{i}(a, \mathbf{x})$ is the probability that $i$ plays action $a$ under Best Reply dynamics given the population state $\mathbf{x}$. That is

$$
B R_{i}(a, \mathbf{x})= \begin{cases}1 & \text { if } a \in \arg \max _{b \in X} U_{i}\left(b, \mathbf{x}_{-i}\right) \\ 0 & \text { otherwise. }\end{cases}
$$

This model of mistakes is similar to that in Freidlin and Wentzell [42], Kandori et al. [57], Young [113], Catoni [26] and Beggs [14] among others. The above dynamics defines a Markov chain on a finite state space $\mathbf{X}$ whose transition matrix $P_{\varepsilon}$ is defined by transition probabilities $P_{\mathcal{E}}(\mathbf{x}, \mathbf{y})$,

$$
P_{\varepsilon}(\mathbf{x}, \mathbf{y})=\prod_{i=1}^{n} \mathbb{P}_{i}\left(y^{i}, \mathbf{x}\right)
$$

\subsubsection{Closed communication classes and basins of attraction}

The perturbed Markov chain $\left(\mathbf{X}, P_{\mathcal{\varepsilon}}\right)$ has a unique invariant (stationary) distribution $\pi_{\varepsilon}=\lim _{t \rightarrow \infty} \mathbf{q}_{0} P_{\varepsilon}^{t}$, where $\mathbf{q}_{t}$ is the vector of probability mass functions at period $t$. The stationary distribution describes the amount of time the process spends in each state in the long-run. The closed communication classes of $\left(\mathbf{X}, P_{\varepsilon}\right)$ are also the closed communication classes of the identical process $(\mathbf{X}, P)$ without mistakes. $^{2}$ In the coordination game of Table 12 above for example, the limit sets include the singleton sets in which all players play strategy $a$ and in which they all play strategy $b$. Generally, the limit sets of $(\mathbf{X}, P)$ can include sets that are cycles and those in which players use different strategies. When the interactions are governed by a social network, the

2 A set $\Omega \subset \mathbf{X}$ is a closed communication class of $(\mathbf{X}, P)$ if $\forall \mathbf{y} \in \Omega, \mathbb{P}\left(\mathbf{x}_{t+1} \in \Omega \mid \mathbf{x}_{t}=\mathbf{y}\right)=1$, and that $\forall \mathbf{y}, \mathbf{z} \in \Omega$, there exists a $\tau>0$ such that $\mathbb{P}\left(\mathbf{x}_{t+\tau}=\mathbf{z} \mid \mathbf{x}_{t}=\mathbf{y}\right)>0$. 
number of closed communication classes is enhanced. In particular, singleton closed communication classes in which strategies co-exists and different cohesive subgroups adopt different strategies may exist. The closed communication classes of $(\mathbf{X}, P)$ that results in the long-run depends on the initial state of the process. We denote the typical limit set of $(\mathbf{X}, P)$ and hence recurrent class of $\left(\mathbf{X}, P_{\varepsilon}\right)$ by $\Omega$, and by $\Omega$ for the set of all such subsets.

\subsection{EXPECTED WAiting Time}

In this section, we provide bounds for expected waiting times between closed communication classes. The results we establish in this section are also crucially relevant for the analysis of convergence times in the next section. The characterization of expected waiting times utilizes the concepts of hitting time for finite Markov chains. In particular, we make us of Lemma 3.4 in Freidlin and Wentzell [42, Chapter 6] and Lemma 4 in Catoni [26]. Before stating the Lemma the following notations and definitions are necessary.

Given the Markov chain $\left(\mathbf{X}, P_{\varepsilon}\right)$, let $W \subset \mathbf{X}$ be a subset of $\mathbf{X}$ and $W^{c}=\mathbf{X} \backslash W$ its complement. For any oriented graph $g \subset \mathbf{X} \times \mathbf{X}$ and any $\mathbf{x} \in \mathbf{X}$, write $g(\mathbf{x})=$ $\{\mathbf{y}:(\mathbf{x}, \mathbf{y}) \in g\}$ denote the immediate successors of $\mathbf{x}$. More generally $g^{n}(\mathbf{x})=$ $\bigcup_{\mathbf{y} \in g^{n-1}(\mathbf{y})} g(\mathbf{x})$.

Definition 8: Let $G(W)$ be the set of oriented graphs $g \subset \mathbf{X} \times \mathbf{X}$ satisfying:

1. for any $\mathbf{x} \in \mathbf{X}, \# g(\mathbf{x})=\mathbf{1}_{W^{c}}$ (that is no arrows start from $W$ and exactly on arrow starts from each state outside of $W$ ),

2. for any $\mathbf{x} \in \mathbf{X}, \mathbf{x} \notin \mathcal{O}_{g}(\mathbf{x})$, where $\mathcal{O}_{g}(\mathbf{x})=\bigcup_{n=1}^{+\infty} g^{n}(\mathbf{x})$ is the set of points that can be reached from $\mathbf{x}$; the orbit of $\mathbf{x}$ under $g$ (that is $g$ has no loops).

Definition 9: For any $\mathbf{x} \in \mathbf{X}$ and $\mathbf{y} \in W$, write

$$
G_{\mathbf{x}, \mathbf{y}}(W)= \begin{cases}\left\{g \in G(W): \mathbf{y} \notin \mathcal{O}_{g}(\mathbf{x})\right\} & \text { if } \mathbf{x} \in W^{c} \\ G(W) & \text { if } \mathbf{x}=\mathbf{y} \\ \varnothing & \text { if } \mathbf{x} \in W \backslash\{\mathbf{y}\}\end{cases}
$$

That is $G_{\mathbf{x}, \mathbf{y}}(W)$ is the set of graphs $g \in G(W)$ linking $\mathbf{x}$ to $\mathbf{y}$. The following Lemma is proved in Freidlin and Wentzell [42] and in Catoni [26]. 
Lemma 9: Let $T(W)=\inf \left\{t \geq 0: \mathbf{x}_{t} \in W\right\}$ be the first time the chain reaches $W$. For any $W \neq \varnothing$, any $\mathbf{x} \in W^{c}$,

$$
\mathbb{E}\left[T(W) \mid \mathbf{x}_{0}=\mathbf{x}\right]=\left(\sum_{\mathbf{y} \in W^{c}} \sum_{g \in G_{\mathbf{x}, \mathbf{y}}(W \cup\{\mathbf{y}\})} P_{\mathcal{\varepsilon}}(g)\right)\left(\sum_{g \in G(W)} P_{\mathcal{\varepsilon}}(g)\right)^{-1}
$$

where $\mathbb{E}$ stands for expectation and $P_{\mathcal{\varepsilon}}(g)=\prod_{(\mathbf{y}, \mathbf{z}) \in g} P_{\mathcal{\varepsilon}}(\mathbf{y}, \mathbf{z})$.

For $\mathbf{x} \neq \mathbf{y}$, the graph $G_{\mathbf{x}, \mathbf{y}}(W \cup\{\mathbf{y}\})$ is the set of all $G(W)$ graphs that link $\mathbf{x}$ to $\mathbf{y}$. This implies that if $W^{c}$ is a singleton set, then the component $\sum_{\mathbf{y} \in W^{c}} \sum_{g \in G_{\mathbf{x}, \mathbf{y}}(W \cup\{\mathbf{y}\})} P_{\varepsilon}(g)$ on the right hand side of (4.3.1) is equal to one.

The above definitions and Lemma 9 directly extend to the Markov chains $\left(\Omega, P_{\varepsilon}\right)$ and $\left(\tilde{\Omega}, \tilde{P}_{\mathcal{E}}\right)$. That is for $\left(\Omega, P_{\mathcal{\varepsilon}}\right), P_{\mathcal{\varepsilon}}(g)=\prod_{\left(\Omega, \Omega^{\prime}\right) \in g} P_{\mathcal{E}}\left(\Omega, \Omega^{\prime}\right)$ in (4.3.1) above, and for $\left(\tilde{\Omega}, \tilde{P}_{\mathcal{E}}\right)$

$$
\mathbb{E}\left[T(W) \mid \tilde{\Omega}_{0}=\tilde{\Omega}\right]=\left(\sum_{\tilde{\Omega}^{\prime} \in W^{c}} \sum_{g \in G_{\tilde{\Omega}, \Omega^{\prime}}\left(W \cup\left\{\tilde{\Omega}^{\prime}\right\}\right)} \tilde{P}_{\mathcal{\varepsilon}}(g)\right)\left(\sum_{g \in G(W)} \tilde{P}_{\mathcal{\varepsilon}}(g)\right)^{-1}
$$

for any $W \neq \varnothing$, any $\tilde{\Omega}^{\prime} \in W^{c}$, where $\tilde{P}_{\mathcal{\varepsilon}}(g)=\prod_{\left(\tilde{\Omega}, \tilde{\Omega}^{\prime}\right) \in g} \tilde{P}_{\mathcal{\varepsilon}}\left(\tilde{\Omega}, \tilde{\Omega}^{\prime}\right)$.

A complete characterization of the expected waiting times therefore requires establishing the structure of the probabilities $P_{\mathcal{E}}\left(\Omega, \Omega^{\prime}\right)$. As in Chapters 2 and 3 above, it is convenient to split the transition $\Omega \rightarrow \Omega^{\prime}$ into two transitions, from $\Omega \rightarrow \partial \Omega^{\prime}$ and $\partial \Omega^{\prime} \rightarrow \Omega^{\prime}$.

Given the above probability structure, the cost is formally given by

$$
C\left(\Omega, \partial \Omega^{\prime}\right)=-R\left(\Omega, \partial \Omega^{\prime}\right) \ln \mathscr{P}=-R\left(\Omega, \partial \Omega^{\prime}\right)\left(\ln m^{-1}-\beta\right)
$$

The following Lemma provides an approximation for the exit probabilities rather the lower and upper bounds as in Chapters 2 and 3

LEMMA 10: For any pair $\Omega$ and $\Omega^{\prime}$ of closed communication classes, the maximum probability $P_{\mathcal{E}}\left(\Omega, \partial \Omega^{\prime}\right)$ from a state in $\Omega$ to a state in $\partial \Omega^{\prime}$ in a single time step is given by

$$
P_{\varepsilon}\left(\Omega, \partial \Omega^{\prime}\right) \approx K_{N} \exp \left\{-N\left(c\left(\Omega, \partial \Omega^{\prime}\right)+F\left(r\left(\Omega, \partial \Omega^{\prime}\right)\right)\right)\right\}
$$

where $F(r)=r \ln r-(1-r) \ln (1-r)$.

Proof. See Appendix 4.9.1

Similarly, from Chapters 2 and 3 we can approximate the contagion probabilities for $\beta$ sufficiently large as

$$
P_{\varepsilon}(\partial \Omega, \Omega) \approx \exp \left\{K_{\Omega} \ln \left(1-\lambda_{2}(G)\right)\right\},
$$


such that

$$
P_{\mathcal{E}}\left(\Omega, \Omega^{\prime}\right) \approx K_{N} \exp \left\{-N\left(c\left(\Omega, \partial \Omega^{\prime}\right)+F\left(r\left(\Omega, \partial \Omega^{\prime}\right)\right)\right)+\gamma\left(\Omega^{\prime}\right)\right\}
$$

where $\gamma\left(\Omega^{\prime}\right)=K_{\Omega^{\prime}} \ln \left(1-\lambda_{2}(G)\right)$.

THeorem 3: For any $W \neq \varnothing$, any $\Omega \in W$

$$
\mathbb{E}\left[T\left(W^{c}\right) \mid \mathbf{x}_{0} \in \Omega\right] \leq K_{T} \exp \left\{\min _{g \in G\left(W^{c}\right)} \psi(g)-\min _{\Omega^{\prime} \in W} \min _{g \in G_{\Omega, \Omega^{\prime}}\left(W \cup\left\{\Omega^{\prime}\right\}\right)} \psi g\right\}
$$

where $\psi(g)=N(c(g)+F(r(g)))-\gamma(g), c(g)=\sum_{\left(\Omega, \Omega^{\prime}\right) \in g} c\left(\Omega, \partial \Omega^{\prime}\right)$, $\gamma(g)=\sum_{\left(\Omega, \Omega^{\prime}\right) \in g} \gamma\left(\Omega^{\prime}\right)$ and $F(r(g))=\sum_{\left(\Omega, \Omega^{\prime}\right) \in g} F\left(r\left(\Omega, \partial \Omega^{\prime}\right)\right)$.

Proof. See Appendix 4.9.2

Through the analysis that follows we let $M_{W}(\Omega)=\min _{\Omega^{\prime} \in W} \min _{g \in G_{\Omega, \Omega^{\prime}}}\left(W \cup\left\{\Omega^{\prime}\right\}\right) \psi(g)$, $M_{W}=\min _{g \in G\left(W^{c}\right)} \psi(g)$ and $H_{W}(\Omega)=M_{W}-M_{W}(\Omega)$. From (4.3.7), for finite and relatively small values of $\beta$, two factors influence the expected waiting time for evolution in networks. The first is the radii (resistances) of closed communication classes, which are determined by the underlying payoff and network topology. The second factor is the spectral property of the underlying network. For a given model of mistakes and equal basins of attraction, highly connected networks exhibit lower expected waiting times than sparsely connected networks. This result follows from the discussion about second largest eigenvalues of graphs above. We elaborate on this point further below for specific families of networks. As $\beta$ becomes exceedingly large, the effect of the spectral gap of the underlying network on the expected waiting time becomes negligible, that is

$$
\begin{aligned}
\lim _{\beta \rightarrow \infty} \frac{1}{\beta} \ln \mathbb{E}\left(T\left(W^{c}\right) \mid \mathbf{x}_{0} \in \Omega\right) & =N\left(\min _{g \in G\left(W^{c}\right)} r(g)-\min _{\Omega^{\prime} \in W} \min _{g \in G_{\Omega, \Omega^{\prime}}\left(W \cup\left\{\Omega^{\prime}\right\}\right)} r(g)\right) \\
& =\min _{g \in G\left(W^{c}\right)} R(g)-\min _{\Omega^{\prime} \in W} \min _{g \in G_{\Omega, \Omega^{\prime}}\left(W \cup\left\{\Omega^{\prime}\right\}\right)} R(g)
\end{aligned}
$$

where $R(g)=\sum_{\left(\Omega, \Omega^{\prime}\right) \in g} R\left(\Omega, \partial \Omega^{\prime}\right)$. This is then equivalent to limit results in Beggs [14]. A more detailed examination of the expected waiting times for various families of networks is performed in sections that follow. Here, we provide a simple example demonstrating its computation.

\subsubsection{Example}

Consider a $3 \times 3$ matrix coordination game in which there are three singleton closed communication classes a, b, c. Figure 5 summarizes the transitions among 
closed communication classes where the weight on each arrow is the quantity $\psi\left(\Omega, \Omega^{\prime}\right)=$ $N\left(c\left(\Omega, \partial \Omega^{\prime}\right)+F\left(r\left(\Omega, \partial \Omega^{\prime}\right)\right)\right)-\gamma\left(\Omega^{\prime}\right)$.

Corollary 2: Given $\psi\left(\Omega, \Omega^{\prime}\right)$ for each pair $\left(\Omega, \Omega^{\prime}\right)$ in Figure 5,

(i) if $W=\{\mathbf{a}, \mathbf{b}\}$, then $\mathbb{E}\left(T\left(W^{c}\right) \mid \mathbf{x}_{0}=\mathbf{a}\right) \leq K_{T} \exp (3.33 N)$,

(ii) if $W=\{\mathbf{a}, \mathbf{c}\}$, then $\mathbb{E}\left(T\left(W^{c}\right) \mid \mathbf{x}_{0}=\mathbf{c}\right) \leq K_{T} \exp (4.96 N)$.

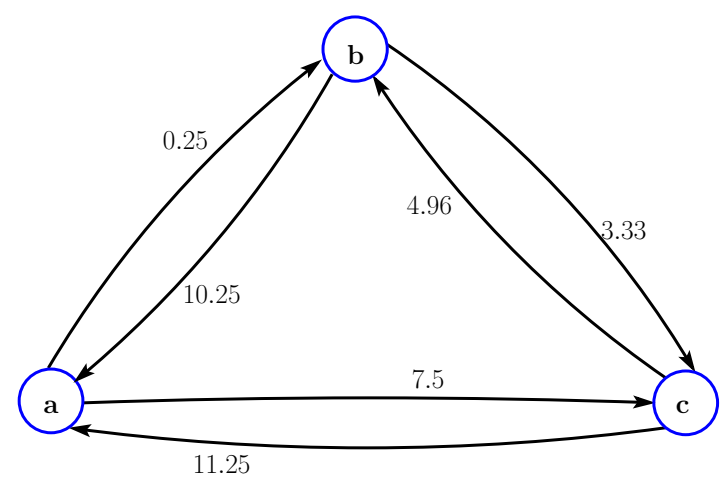

Figure 5: The weight on each arrow is the quantity $N\left(c\left(\Omega, \partial \Omega^{\prime}\right)+F\left(r\left(\Omega, \partial \Omega^{\prime}\right)\right)\right)-$ $\gamma\left(\Omega^{\prime}\right)$

Proof. When $W=\{\mathbf{a}, \mathbf{b}\}$, the least cost graph linking $W$ to $\mathbf{c}$ is $\mathbf{a} \rightarrow \mathbf{b} \rightarrow \mathbf{c}$. Implying that $M_{W}=3.58$. The graph that minimizes $M_{W}(\mathbf{a})$ is $\mathbf{a} \rightarrow \mathbf{b}$, hence $M_{W}(\mathbf{a})=0.25$. Implying that $H_{W}(\mathbf{a})=M_{W}-M_{W}(\mathbf{a})=3.33$.

When $W=\{\mathbf{a}, \mathbf{c}\}$, we have $\{\mathbf{a} \rightarrow \mathbf{b}, \mathbf{c} \rightarrow \mathbf{b}\}$ to be the least cost graph linking $W$ to $\mathbf{b}$. Hence $M_{W}=3.58$. The graph $\mathbf{a} \rightarrow \mathbf{b}$ minimizes $M_{W}(\mathbf{c})$, hence $M_{W}(\mathbf{c})=0.25$. Implying that $H_{W}(\mathbf{c})=4.96$. The respective expected waiting times directly follow by substituting for $H_{W}(\Omega)$.

\subsection{CONVERGENCE TIME}

In this section, we derive bounds for the convergence time as a measure of how fast (slow) equilibrium selection occurs. We employ the concept of mixing time for finite Markov chains, which is a fairly well documented concept. Mixing times can be defined in terms of various distance measures such as $L^{p}$-norms, but here we opt for the most intuitive distance measure; the total variation distance. Total variation 
distance is a probabilistic measure of the difference between two distributions. Formally, let $v$ and $\pi$ be two probability distributions on the state space $\mathbf{X}$. Then the total variation distance is defined by

$$
\|v-\pi\|_{T V}=\max _{\chi \subset \mathbf{X}}|v(\chi)-\pi(\chi)| .
$$

That is, the maximum difference between the probabilities assigned to a single event by the two distributions. Total variation distance can be equivalently expressed as,

$$
\|v-\pi\|_{T V}=\frac{1}{2} \sum_{\mathbf{x} \in \mathbf{X}}|\nu(\mathbf{x})-\pi(\mathbf{x})| .
$$

The validity of relation (4.4.1) can be demonstrated geometrically using Figure 6. First notice that both $v$ and $\pi$ being probabilistic measures implies that $v\left(\chi^{*}\right)$ is the area under the curve $v$ but to the left of the vertical dashed line $\mathbf{x}=\chi^{*}$. Since for both distributions the total area under the curve is one, it follows that the area of regions $A$ and $B$ are equal. For these two specific distributions (of Figure 6), the total variation distance between them is thus the area of the region $A$, which by symmetry equals the area of region $B$. The quantity $\sum_{\mathbf{x} \in \mathbf{X}}|v(\mathbf{x})-\pi(\mathbf{x})|$ in (4.4.1) is the area of both regions $A$ and $B$, hence the factor of $\frac{1}{2}$ in the equality. Relation (4.4.1) is essentially true for all forms of probabilistic distributions.

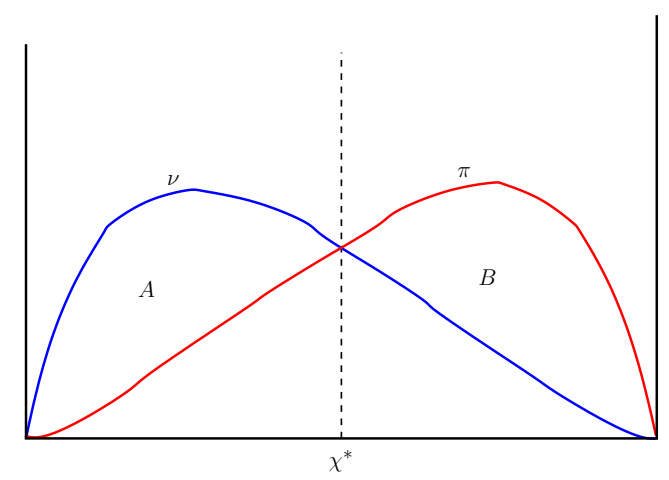

Figure 6: The total variation distance between distributions $v$ and $\pi$

The convergence time $T_{\mathcal{C}}\left(P_{\varepsilon}\right)$ of the process $P_{\varepsilon}$ is then formally defined as,

$$
T_{\mathcal{C}}\left(P_{\varepsilon}\right)=\min \left\{t:\left\|\mathbf{q}_{0} P_{\varepsilon}^{t^{\prime}}-\pi\right\| \leq \omega ; \forall t^{\prime}>t\right\}
$$

where $\mathbf{q}_{0}$ is the initial distribution. We also write $\mathbf{q}_{t}$ for the distribution at $t$. For a given initial distribution, the convergence time is therefore the time it takes the process to get close to its stationary distribution. 
The usual approach in the study of mixing times is to first derive the lower and upper bounds in terms of the second largest eigenvalue of the transition matrix of the chain. The analysis then focuses of establishing the properties of the second eigenvalue, which can be done in several ways. One technique is by making use of the well known Cheeger's inequality $[3,5,76,108]$. That is if $\mu_{2}$ is the second largest eigenvalue of the $P_{\mathcal{\varepsilon}}$, then

$$
1-2 \phi\left(P_{\varepsilon}\right) \leq \mu_{2} \leq 1-\frac{\phi\left(P_{\varepsilon}\right)^{2}}{2}
$$

where $\phi\left(P_{\mathcal{\varepsilon}}\right)$, the conductance of $P_{\mathcal{\varepsilon}}$, is given by $\phi\left(P_{\mathcal{\varepsilon}}\right)=\min _{W \subset \mathbf{X}} \phi\left(P_{\mathcal{E}}, W\right)$. And,

$$
\sum_{\mathbf{x} \in W} \pi(\mathbf{x}) \leq \frac{1}{2}
$$

$$
\phi\left(P_{\mathcal{\varepsilon}}, W\right)=\frac{1}{\sum_{\mathbf{x} \in W} \pi(\mathbf{x})} \sum_{\mathbf{x} \in W} \sum_{\mathbf{y} \in W^{c}} \pi(\mathbf{x}) P_{\mathcal{\varepsilon}}(\mathbf{x}, \mathbf{y}) .
$$

The second technique involves the use of the concept of multicommodity flows. The details of both of these characterizations can be found in Diaconis and Stroock [33] and Sinclair [107]. These characterizations are however not readily computable for large state space Markov chains. Moreover, they are not directly intuitive with respect to how the underlying game and network affect convergence rates. Here, we express the upper bounds for convergence time in terms of the maximum expected waiting time. This enables us to directly apply some of the well known results on expected waiting times for stochastic evolutionary processes, together with what we established in Section 4.3 above, to the analysis of convergence time. It also ensures that we can focus on the properties of the reduced Markov chain $\left(\tilde{\Omega}, \tilde{P}_{\mathcal{E}}\right)$ rather than the original cumbersome chain. The following theorem establishes the upper bound for the convergence time.

Theorem 4: For any $W \neq \varnothing$, any $\tilde{\Omega} \in W$ and $\tilde{\Omega}^{\prime} \in W^{c}$, let $\mathscr{T}=\max _{\tilde{\Omega}, \tilde{\Omega}^{\prime}} \mathbb{E}\left[T\left(\tilde{\Omega}^{\prime}\right) \mid \mathbf{x}_{0} \in\right.$ $\tilde{\Omega}]$. Let also $\tilde{\pi}$ be the stationary distribution of $\tilde{P}_{\mathcal{\varepsilon}}$. Then the convergence time $T_{\mathcal{c}}$ is bounded from above by

$$
T_{\mathcal{c}} \leq \frac{K_{c}}{2 \tilde{\pi}_{\max }^{2}}\left(\ln \tilde{\pi}_{\min }^{-1}+\ln (2 \omega)^{-1}\right) \mathscr{T}
$$

where $K_{c}$ is some constant, $\tilde{\pi}_{\min }=\min _{\tilde{\Omega} \in \tilde{\Omega}} \tilde{\pi}(\tilde{\Omega})$ and $\tilde{\pi}_{\max }=\max _{\tilde{\Omega} \in \tilde{\Omega}} \tilde{\pi}(\tilde{\Omega})$.

Proof. A detailed proof is provided in Appendix 4.9.3 whose sketch is as follows. The proof focuses on the dynamics of the reduced chain $\left(\tilde{\Omega}, \tilde{P}_{\varepsilon}\right)$. The first step involves showing that

$$
T_{c} \leq \frac{1}{\ln \tilde{\mu}_{2}}\left(\ln \tilde{\pi}_{\min }^{-1}+\ln (2 \omega)^{-1}\right) \approx \frac{1}{1-\tilde{\mu}_{2}}\left(\ln \tilde{\pi}_{\min }^{-1}+\ln (2 \omega)^{-1}\right)
$$


where $\tilde{\mu}_{2}$ is the second largest eigenvalue of the $\tilde{P}_{\mathcal{\varepsilon}}$. The approximation is valid for values of $\tilde{\mu}_{2}$ close to one, which is true for the type of dynamics we considered in this Chapter (a consequence of small perturbations). Inequality (4.4.6) appears in the literature of finite Markov chains in closely related forms (e.g. Diaconis and Stroock [33]).

The next step of the proof is then to show that

$$
\frac{1}{1-\tilde{\mu}_{2}} \leq \frac{K_{c}}{2 \tilde{\pi}_{\max }^{2}} \mathscr{T}
$$

The right hand side of (4.4.7) follows from a set of Theorems from Bremaud [23] and Takacs [110]. More specifically, as a corollary of Takacs [110, Theorem 1.2 and Proposition 3.1], the expected waiting time between any two given subsets say $\tilde{\Omega}$ and $\tilde{\Omega}^{\prime}$ is given by

$$
\mathbb{E}\left(T\left(\tilde{\Omega}^{\prime}\right) \mid \mathbf{x}_{0} \in \tilde{\Omega}\right)=\sum_{k=2}^{\# \tilde{\Omega}} \frac{1}{1-\tilde{\mu}_{k}}\left(\tilde{v}_{\tilde{\Omega}^{\prime}}^{2}(k)-\tilde{v}_{\tilde{\Omega}}(k) \tilde{v}_{\tilde{\Omega}^{\prime}}(k)\right)
$$

where $\tilde{\mathbf{v}}(k)$ is the eigenvector corresponding to $\tilde{\mu}_{k}$, such that $\tilde{v}_{l}(k)$ is the $l$ th element of $\tilde{\mathbf{v}}(k)$. We thus have that

$$
\mathscr{T}=\max _{\tilde{\Omega}, \tilde{\Omega}^{\prime}} \mathbb{E}\left(T\left(\tilde{\Omega}^{\prime}\right) \mid \mathbf{x}_{0} \in \tilde{\Omega}\right)=\max _{\tilde{\Omega}, \tilde{\Omega}^{\prime}} \sum_{k=2}^{\# \tilde{\Omega}} \frac{1}{1-\tilde{\mu}_{k}} \tilde{v}_{\tilde{\Omega}^{\prime}}(k)\left(\tilde{v}_{\tilde{\Omega}^{\prime}}(k)-\tilde{v}_{\tilde{\Omega}}(k)\right)
$$

We can then exploit the properties of the eigenvectors of finite state Markov chains. The eigenvector corresponding to the first eigenvalues (first eigenvector) is an all one vector. The other remaining eigenvectors can be used to detect (or partition the state space into) almost invariant subsets (e.g. Froyland [43] and Billings and Schwartz [17]). This concept is familiar in the literature of cluster detection. More generally, the second eigenvector can be used to partition the chain into two almost invariant subsets, the third eigenvector separates the chain into three almost invariant subsets, and so forth. The eigenvector corresponding to the \# $\Omega$ th eigenvalue of $P_{\varepsilon}$ and $\tilde{P}_{\varepsilon}$ therefore partitions the chain into $\# \mathbf{\Omega}$ almost invariant subsets, which are basically the respective basins of attraction.

The detection process proceeds by identifying the minima (regions of negative values) and maxima (peaks and regions of positive values) of the eigenvectors. The second eigenvector therefore has one maximum and one minimum. Since the maximum of the right hand side of (4.4.9) occurs when the differences $\tilde{v}_{\tilde{\Omega}^{\prime}}(k)-\tilde{v}_{\tilde{\Omega}}(k)$ are maximum, we can thus approximate $\max _{\tilde{\Omega}, \tilde{\Omega}^{\prime}}\left(\tilde{v}_{\tilde{\Omega}^{\prime}}(k)-\tilde{v}_{\tilde{\Omega}}(k)\right) \approx 2 \max _{\tilde{\Omega} \in \tilde{\Omega}} \tilde{v}_{\tilde{\Omega}}(k)$ for all $k \geq 2$. Note that each point of $\tilde{\Omega}$ for which $\tilde{\mathbf{v}}(k)$ attains its minima and maxima are also those at which the stationary distribution $\tilde{\pi}$ attains its maxima. It follows that 
there exist constants $K_{k}$ for $k \geq 2$ such that $\max _{\tilde{\Omega} \in \tilde{\Omega}}\left|\tilde{v}_{\tilde{\Omega}}(2)\right|=K_{2} \max _{W \subset \tilde{\Omega}} \tilde{\pi}(W), \cdots$, $\max _{\tilde{\Omega} \in \tilde{\Omega}}\left|\tilde{v}_{\tilde{\Omega}}(\# \tilde{\mathbf{\Omega}})\right|=K_{\# \tilde{\Omega}} \max _{\tilde{\Omega} \in \tilde{\Omega}} \tilde{\pi}(\tilde{\Omega})$. Since $\max _{W \subset \tilde{\Omega}} \tilde{\pi}(W) \geq \cdots \geq \max _{\tilde{\Omega} \in \tilde{\Omega}} \tilde{\pi}(\tilde{\Omega})=$ $\tilde{\pi}_{\max }$, we then have that

$$
\mathscr{T}=\max _{\tilde{\Omega}, \tilde{\Omega}^{\prime}} \sum_{k=2}^{\# \tilde{\Omega}} \frac{1}{1-\tilde{\mu}_{k}} \tilde{v}_{\tilde{\Omega}^{\prime}}(k)\left(\tilde{v}_{\tilde{\Omega}^{\prime}}(k)-\tilde{v}_{\tilde{\Omega}}(k)\right) \geq 2 K_{\# \tilde{\Omega}^{\prime}} \tilde{\pi}_{\max }^{2} \sum_{k=2}^{\# \tilde{\Omega}} \frac{1}{1-\tilde{\mu}_{k}} \geq 2 K_{\# \tilde{\Omega}} \tilde{\pi}_{\max }^{2} \frac{1}{1-\tilde{\mu}_{2}}
$$

Substituting for $\mathscr{T}$ in (4.4.6) then completes the proof, where $K_{c}=\frac{1}{K_{\# \tilde{\Omega}}}$.

Theorem 4 establishes an upper bound for the convergence time in terms of the measure of the reduced chain, the minimum and maximum values of the stationary distribution and the maximum expected waiting time. An explicit characterization of the stationary distributions for finite state Markov chains is performed by Freidlin and Wentzell [42] and Catoni [26]. More generally, for each $\tilde{\Omega} \in \tilde{\mathbf{\Omega}}$,

$$
\tilde{\pi}(\tilde{\Omega})=\left(\sum_{g \in G(\{\tilde{\Omega}\})} \tilde{P}_{\mathcal{\varepsilon}}(g)\right)\left(\sum_{\tilde{\Omega}^{\prime} \in \tilde{\Omega}} \sum_{g \in G\left(\left\{\tilde{\Omega}^{\prime}\right\}\right)} \tilde{P}_{\mathcal{E}}(g)\right)^{-1}
$$

where $\tilde{P}_{\mathcal{E}}(g)=\prod_{\left(\tilde{\Omega}, \tilde{\Omega}^{\prime}\right) \in g} \tilde{P}_{\mathcal{E}}\left(\tilde{\Omega}, \tilde{\Omega}^{\prime}\right)$. From the definition of $G(W)$ graphs above, $G(\{\tilde{\Omega}\})$ is the set of all spanning trees in which there exists a unique directed path from every $\tilde{\Omega}^{\prime} \neq \tilde{\Omega}$ to $\tilde{\Omega}$, also known as $\tilde{\Omega}$-trees. The probability $\tilde{P}_{\varepsilon}\left(\tilde{\Omega}, \tilde{\Omega}^{\prime}\right)=$ $P_{\mathcal{E}}(\partial \Omega, \Omega) P_{\mathcal{E}}\left(\Omega, \partial \Omega^{\prime}\right)$, and by making use of (4.3.5) and (4.3.4),

$$
\tilde{P}_{\mathcal{E}}\left(\tilde{\Omega}, \tilde{\Omega}^{\prime}\right)=K_{N} \exp \left\{-N\left(c\left(\Omega, \partial \Omega^{\prime}\right)+F\left(r\left(\Omega, \partial \Omega^{\prime}\right)\right)\right)+\gamma(\Omega)\right\},
$$

such that

$$
\frac{1}{\tilde{\pi}(\tilde{\Omega})} \leq K_{\pi} \exp \{\varphi(\tilde{\Omega})\}
$$

where

$\varphi(\tilde{\Omega})=\min _{g \in G(\{\tilde{\Omega}\})}(N[c(g)+F(r(g))]-\gamma(g))-\min _{\tilde{\Omega}^{\prime} \in \tilde{\Omega}} \min _{g \in G\left(\left\{\tilde{\Omega}^{\prime}\right\}\right)}(N[c(g)+F(r(g))]-\gamma(g))$ and $K_{\pi}$ is a constant.

From (4.4.12) it follows that the long-run stable state or subset (corresponding to $\tilde{\pi}_{\text {max }}$ ) lies in the basin of attraction $\tilde{\Omega}^{*}$ for which $\varphi\left(\tilde{\Omega}^{*}\right)=0$. The state or subset corresponding to $\tilde{\pi}_{\min }$ is that for which $\varphi(\tilde{\Omega})$ is maximum. The following Corollary demonstrates how to compute convergence times.

CoROLlary 3: Consider the an evolutionary process for which the values of the quantity $N\left[c\left(\Omega, \partial \Omega^{\prime}\right)+F\left(r\left(\Omega, \partial \Omega^{\prime}\right)\right)\right]-\gamma(\Omega)$ are as depicted in Figure 7. Then

$$
T_{c} \leq \kappa_{1}\left(\kappa_{2}+4.38 N\right) \exp (4.96 N),
$$

where $\kappa_{1}=\frac{1}{2} K_{c} K_{T} K_{\pi}^{2}$ and $\kappa_{2}=\ln \left(2 K_{\pi}\right)-1$ are universal constants. 


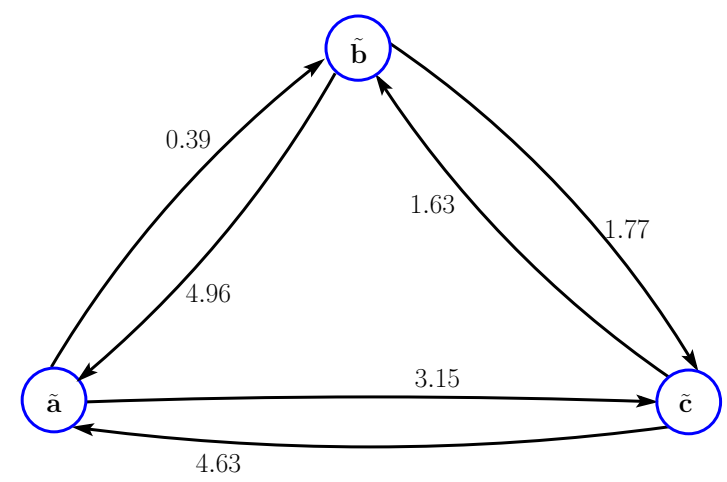

Figure 7: The weight on each arrow is the quantity $N\left(c\left(\Omega, \partial \Omega^{\prime}\right)+F\left(r\left(\Omega, \partial \Omega^{\prime}\right)\right)\right)-$ $\gamma(\Omega)$

Proof. To compute the maximum and minimum values for the stationary distributions, we need to identify and compute the values of the minimum and maximum cost $\tilde{\Omega}$-tree. From Figure 7 , the least cost tree is the $\tilde{\mathbf{b}}$-tree $\{\tilde{\mathbf{a}} \rightarrow \tilde{\mathbf{b}}, \tilde{\mathbf{c}} \rightarrow \tilde{\mathbf{b}}\}$, making $\mathbf{b}$ the long-run stable state (which in this specific case also corresponds to the stochastically stable state). The respective total cost of $\tilde{\mathbf{b}}$-tree is 2.02. The maximum cost tree is the $\tilde{\mathbf{a}}$-tree with cost of 6.4 , such that $\varphi(\tilde{\mathbf{a}})=6.4-2.02=4.38$. Hence $\tilde{\pi}_{\min }^{-1} \leq K_{\pi} \exp (4.38 N)$.

The maximum expected waiting time corresponds to the hitting time of the path $W=\{\mathbf{b}, \mathbf{c}\} \rightarrow\{\mathbf{a}\}$ such that the maximum $M_{W}=1.63+4.96=6.59$ and $M_{W}(\mathbf{c})=$ 1.63. Implying that $\mathscr{T} \leq K_{T} \exp (4.96 N)$. Substituting for $\tilde{\pi}_{\min }^{-1}, \mathscr{T}$ and $\tilde{\pi}_{\min }^{-1} \leq K_{\pi}$ yields the result.

\subsection{DETERMINISTIC NETWORKS}

In this section, we focus on stochastic evolution in deterministic networks. We start by considering the case in which strategies can spread through contagion once threshold adoption levels are attained. An action is said to be globally contagious if it can spread to the entire population through best-response once it has been adopted by a small subgroup of players. The focus of analysis in this section is on $2 \times 2$ matrix games with the general structure in Table 12 above and the case in which players care about the average payoffs. The most relevant quantity of any given matrix game for 
the dynamics specified above is the relative payoff gains, which for the game in Table 12 is given by

$$
\eta_{b a}=\frac{U(b, b)-U(a, b)}{U(b, b)-U(a, b)+U(a, a)-U(b, a)}
$$

and $\eta_{a b}=1-\eta_{b a}$. That is $\eta_{b a}$ is the fraction of neighbors that each player requires to play $a$ for that player to do likewise and vise versa for $\eta_{a b}$. We write $\lceil m\rceil$ fo the smallest integer not less than $m$ such that for each $i \in \mathcal{N}$ with respective degree $k_{i}$, the quantity $\left\lceil\eta_{b a} k_{i}\right\rceil$ is the minimum number of neighbors $i$ requires to play $a$ for $i$ to do likewise.

For any given network, there exists a payoff structure and hence values of relative payoff gains $\eta_{a b}$ 's for which contagion is feasible. The maximum values of relative payoffs for which contagion is feasible are called contagion thresholds (see [78] and Chapter 3 above). If $\eta^{*}$ is the contagion threshold for a given network topology, then any action say $a$ for which $\eta_{b a}<\eta^{*}$ for all $b$, will be contagious. Morris [78] provides an explicit characterization of contagion threshold for various families of networks, two of which we study below. Lelarge [68] derives similar conditions for random networks. If the conditions for contagion are not satisfied, then the dynamic process will exhibit additional multiple closed communication classes induced by the network structure. We treat this case in a separate Section 4.7 below.

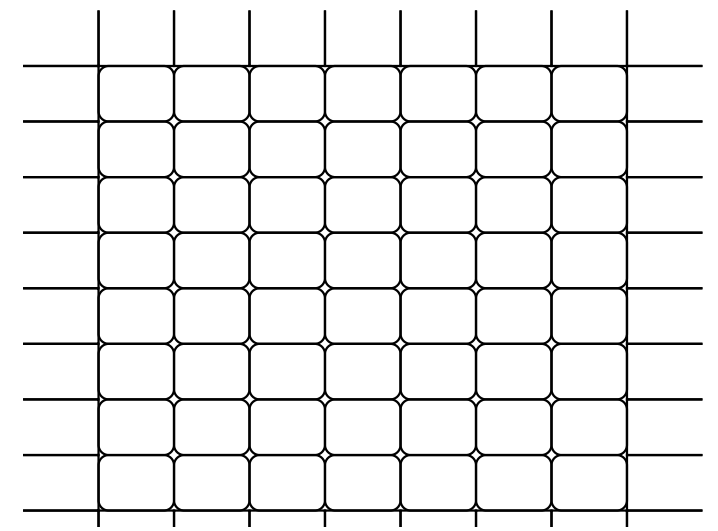

Figure 8: A 2-dimensions nearest neighbor interactions network.

\subsection{1 n-dimensions nearest neighbor interactions}

This family of networks includes as example the line and circle networks as 1dimensional lattices, and the 2-dimension lattice of Figure 8. Morris [78] shows the contagion threshold for such networks to be $\frac{1}{2 n}$; implying that in 1-dimension lattice 
networks if $\eta_{a b}<\frac{1}{2}$ for all $a \neq b$, then $b$ is a contagious action. Similarly for 2dimensional lattice networks, if $\eta_{a b}<\frac{1}{4}$ for all $a \neq b$ then $b$ is a contagious action. And so forth. The following proposition provides convergence rates for this family of networks.

Proposition 8: Let the underlying game be $2 \times 2$ matrix game of Table 12 and let the updating rule be the uniform error probabilities. Let the topology of interactions be an ndimensions nearest neighbor interactions network $G_{n}$ and that $\eta_{a b}<\frac{1}{2 n}$. Then for sufficiently large $N$,

(i) the expected waiting times are;

$$
\begin{gathered}
\mathbb{E}\left[T(\mathbf{b}) \mid \mathbf{x}_{0}=\mathbf{a}\right] \leq \frac{K_{T}}{2} \exp \left\{\beta-\ln \left(1-\lambda_{2}\left(G_{n}\right)\right)\right\} \\
\mathbb{E}\left[T(\mathbf{a}) \mid \mathbf{x}_{0}=\mathbf{b}\right] \leq K_{T} \exp \left\{N\left(\beta-\ln 2^{-1}\right)\right\}
\end{gathered}
$$

(ii) the convergence time is given by

$$
T_{c} \leq \kappa_{1}\left(\kappa_{2}+(N-1)\left(\beta-\ln 2^{-1}\right)-\ln \left(1-\lambda_{2}\left(G_{n}\right)\right)\right) \exp \left\{N\left(\beta-\ln 2^{-1}\right)\right\}
$$

where $\kappa_{1}=K_{c} K_{T} K_{\pi}^{2}$ and $\kappa_{2}=\ln \left(2 K_{\pi}\right)-1$ are universal constants.

Proof. For each $n$-dimensions nearest neighbor interactions network $G_{n}$ if an action say $b$ is contagious in which case $\eta_{a b}<\frac{1}{2 n}$, then each player $i$ requires only one of their neighbor to play $b$ for $i$ to do likewise. Implying that a transition from a to the boundary of the closed communication class $\partial \mathbf{b}$ of $\mathbf{b}$ can be triggered by one player. Such that $r(\mathbf{a}, \partial \mathbf{b})=\frac{1}{N}$. Similarly, the transition $\mathbf{b} \rightarrow \partial \mathbf{a}$ requires all players to simultaneously switch to playing $a$, since each player requires at least $\frac{1}{2} n$ of their neighbors to play $a$ if they are to do likewise. Hence $r(\mathbf{a}, \partial \mathbf{b})=1$.

For $m=2, \mathscr{P}=\frac{1}{2} \exp (-\beta)$ such that $\ln (\mathscr{P})=-\beta+\ln 2^{-1}$. We then have the expected waiting times to be,

$$
\begin{aligned}
\mathbb{E}\left[T(\mathbf{b}) \mid \mathbf{x}_{0}=\mathbf{a}\right] & \leq K_{T} \exp \left\{N\left(\frac{1}{N} \beta-\frac{1}{N} \ln 2^{-1}+F\left(\frac{1}{N}\right)\right)-\ln \left(1-\lambda_{2}\left(G_{n}\right)\right)\right\} \\
& =\frac{K_{T}}{2} \exp \left\{\beta-\ln \left(1-\lambda_{2}\left(G_{n}\right)\right)\right\}
\end{aligned}
$$

where we have used $K_{\mathbf{b}}=1$ since $b$ is globally contagious.

$$
\mathbb{E}\left[T(\mathbf{a}) \mid \mathbf{x}_{0}=\mathbf{b}\right] \leq K_{T} \exp \left\{N\left(\beta-\ln 2^{-1}+F(1)\right)\right\}=K_{T} \exp \left\{N\left(\beta-\ln 2^{-1}\right)\right\}
$$


where $K_{\mathbf{a}}=0$ since $a$ is not globally contagious. In the case of convergence time, we need to compute the minimum and maximum values of the stationary distribution for subsets $\tilde{\Omega}$, and the maximum expected waiting time. From the above expressions of expected waiting, if $\beta$ is sufficiently large (small noise) then $\mathscr{T}=\mathbb{E}\left[T(\mathbf{a}) \mid \mathbf{x}_{0}=\right.$ b] $\leq K_{T} \exp \left\{N\left(\beta-\ln 2^{-1}\right)\right\}$.

For the transition $\partial \mathbf{a} \rightarrow \mathbf{a}$ we have $\mathbb{P}(\partial \mathbf{a}, \mathbf{a}) \approx 1$ since all players have to simultaneously switch to play $a$ for the transition $\mathbf{b} \rightarrow \partial \mathbf{a}$ to occur. We then have

$$
\begin{aligned}
\tilde{P}_{\mathcal{E}}(\tilde{a}, \tilde{b}) & \approx P_{\mathcal{E}}(\partial \mathbf{a}, \mathbf{a}) P_{\mathcal{E}}(\mathbf{a}, \partial \mathbf{b}) \approx P_{\mathcal{\varepsilon}}(\mathbf{a}, \partial \mathbf{b}) \\
& \leq \exp \left\{-N\left(\frac{1}{N} \beta-\frac{1}{N} \ln 2^{-1}+F\left(\frac{1}{N}\right)\right)\right\}=K_{p} \exp \left\{-\left(\beta-\ln 2^{-1}\right)\right\} .
\end{aligned}
$$

$$
\begin{aligned}
\tilde{P}_{\mathcal{\varepsilon}}(\tilde{b}, \tilde{a}) & \approx P_{\mathcal{\varepsilon}}(\partial \mathbf{b}, \mathbf{b}) P_{\mathcal{E}}(\mathbf{b}, \partial \mathbf{a}) \\
& \leq \exp \left\{-N\left(\beta-\ln 2^{-1}\right)+K_{\mathbf{b}} \ln \left(1-\lambda_{2}\left(G_{n}\right)\right)\right\} .
\end{aligned}
$$

where we can let $K_{\mathbf{b}}=1$ as $b$ is globally contagious. Since for $\beta$ sufficiently large $\beta-$ $\ln 2^{-1}<\left(N\left(\beta-\ln 2^{-1}\right)-K_{\mathbf{b}} \ln \left(1-\lambda_{2}\left(G_{n}\right)\right)\right)$, the long-run stable basin of attraction is then $\tilde{\mathbf{b}}$ and hence the long-run stable state is $\mathbf{b}$. Implying that $\varphi(\tilde{\mathbf{b}})=0$ and $\varphi(\tilde{\mathbf{a}})=$ $N\left(\beta-\ln 2^{-1}\right)-\ln \left(1-\lambda_{2}\left(G_{n}\right)\right)-\left(\beta-\ln 2^{-1}\right)=(N-1)\left(\beta-\ln 2^{-1}\right)-\ln \left(1-\lambda_{2}\left(G_{n}\right)\right)$. Such that

$$
\begin{gathered}
\frac{1}{\tilde{\pi}_{\max }} \equiv \frac{1}{\tilde{\pi}(\tilde{b})} \leq K_{\pi} \\
\frac{1}{\tilde{\pi}_{\min }} \equiv \frac{1}{\tilde{\pi}(\tilde{a})} \leq K_{\pi} \exp \left\{(N-1)\left(\beta-\ln 2^{-1}\right)-\ln \left(1-\lambda_{2}\left(G_{n}\right)\right)\right\}
\end{gathered}
$$

Hence $\ln \tilde{\pi}_{\min }^{-1}=\ln K_{\pi}+(N-1)\left(\beta-\ln 2^{-1}\right)-\ln \left(1-\lambda_{2}\left(G_{n}\right)\right)$. Substituting for $\mathscr{T}$, $\tilde{\pi}_{\min }$ and $\tilde{\pi}_{\max }$ into the expression for $T_{c}$ yields the result.

Proposition 8 demonstrates how the network topology plays a critical role in shaping convergence rates. Unlike in the case of global interactions, local interactions reduce the expected waiting time to and increase the exit time from the long-run stable set. This finding is not entirely surprising as it has also been shown for the case of 1-dimension nearest neighbor interactions network by Ellison [37]. Proposition 8 however provides tighter bounds for general $n$-dimensions nearest neighbor interactions family of networks and does not rely on the assumption of vanishing noise for the proof as in Ellison [37].

Proposition 8 also provides an upper bound for the convergence time for $n$-dimensions nearest neighbor interactions family of networks. From (4.5.4), it follows that for 
any two networks in this family say $G_{n}$ and $G_{n^{\prime}}$ with $n>n^{\prime}$, if the relative payoff gains for both networks is such that $\eta_{a b}<\eta^{*}$ then the convergence time for $G_{n}$ is greater than that for $G_{n^{\prime}}$. This argument derives from the fact that when $n>n^{\prime}$ then $\lambda_{2}\left(G_{n}\right)>\lambda_{2}\left(G_{n^{\prime}}\right)$. The result underscores the importance of high connectivity in enhancing high convergence rates. This however is not necessarily the case when $\eta_{a b}>\eta^{*}$, in which case global contagion is not feasible. We treat this special case as that of step-by-step evolution in Section 4.7 below.

\subsection{2 n-dimensions l-max distance interactions}

The second family of networks we consider is the $n$-dimensions $l$-max distance interactions. That is given an n-dimensional lattice network, each player interacts with all players who are within the distance of $l$ steps away in all directions. See Figure 9 for the case of $n=2$ and $l=1$. Morris [78] provides general expressions for contagion thresholds for this family of networks. We focus on the case of $n=2$ for various values of $l$. In this special case the contagion thresholds are given by $\eta^{*}=\frac{l(2 l+1)}{(2 l+1)^{2}-1}$ for $l=1,2,3, \cdots$. It follows by inspection that if $\eta_{a b}=\eta^{*}$ then

$$
r(\mathbf{a}, \partial \mathbf{b})=\frac{2 l(2 l+1)}{N} \quad \text { for } l=1,2, \cdots
$$

See for example the case of $n=2$ and $l=1$ in Figure 9 where $r(\mathbf{a}, \partial \mathbf{b})=\frac{6}{N}$. Relation (4.5.11) is valid so long as $\eta_{a b}=\eta^{*}$ and that $l<\frac{1}{2} \sqrt{N}$. The quantity $\sqrt{N}$ is the length and width of the 2-dimensional lattice. It follows that for $\eta_{a b}<\eta^{*}, r(\mathbf{a}, \partial \mathbf{b})<\frac{2 l(2 l+1)}{N}$. The following proposition is for the case when $\eta_{a b}=\eta^{*}$.

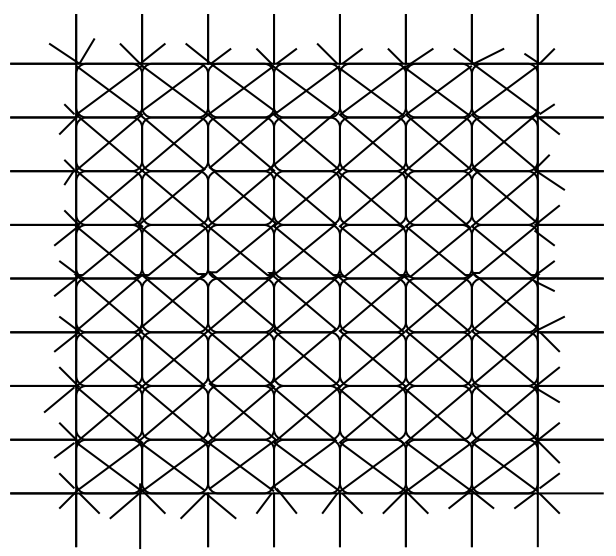

Figure 9: The 2-dimensions 1-max distance interactions 
Proposition 9: Let the underlying game be $2 \times 2$ matrix game of Table 12 and let the updating rule be the uniform error probabilities. If the topology of interactions is the 2dimensions l-max distance interactions $G_{2, l}$ and that $\eta_{a b}=\eta^{*}$ then:

(i) the expected waiting times are;

$$
\mathbb{E}\left[T(\mathbf{b}) \mid \mathbf{x}_{0}=\mathbf{a}\right] \leq K_{T} \exp \{H(\mathbf{b})\}
$$

where $H(\mathbf{b})=2 l(2 l+1)\left(\beta-\ln 2^{-1}\right)+N F\left(\frac{2 l(2 l+1)}{N}\right)-\ln \left(1-\lambda_{2}\left(G_{2, l}\right)\right)$.

$$
\mathbb{E}\left[T(\mathbf{a}) \mid \mathbf{x}_{0}=\mathbf{b}\right] \leq K_{T} \exp \left\{N\left(\beta-\ln 2^{-1}\right)\right\}
$$

(ii) the convergence time is given by

$$
\begin{gathered}
T_{c} \leq \kappa_{1}\left(\kappa_{2}+\varphi(\tilde{\mathbf{a}})\right) \exp \left\{N\left(\beta-\ln 2^{-1}\right)\right\}, \\
\text { where } \varphi(\tilde{\mathbf{a}})=(N-2 l(2 l+1))\left(\beta-\ln 2^{-1}\right)-N F\left(\frac{2 l(2 l+1)}{N}\right)-\ln \left(1-\lambda_{2}\left(G_{2, l}\right)\right) .
\end{gathered}
$$

Proof. First note that when $\eta_{a b} \geq \eta^{*}, r(\mathbf{b}, \partial \mathbf{a}) \approx 1$ for all $l=1,2, \cdots$. By following the same steps as in the proof of Proposition 8 , we have that

$$
\begin{aligned}
\mathbb{E}\left[T(\mathbf{b}) \mid \mathbf{x}_{0}=\mathbf{a}\right] & \leq K_{T} \exp \left\{N\left(\frac{2 l(2 l+1)}{N}\left(\beta-\ln 2^{-1}\right)+F\left(\frac{2 l(2 l+1)}{N}\right)\right)-\ln \left(1-\lambda_{2}\left(G_{n}\right)\right)\right\} \\
& =K_{T} \exp \left\{2 l(2 l+1)\left(\beta-\ln 2^{-1}\right)+N F\left(\frac{2 l(2 l+1)}{N}\right)-\ln \left(1-\lambda_{2}\left(G_{2, l}\right)\right)\right\}
\end{aligned}
$$

$$
\mathbb{E}\left[T(\mathbf{a}) \mid \mathbf{x}_{0}=\mathbf{b}\right] \leq K_{T} \exp \left\{N\left(\beta-\ln 2^{-1}+F(1)\right)\right\}=K_{T} \exp \left\{N\left(\beta-\ln 2^{-1}\right)\right\}
$$

Similarly, for $\eta_{a b}=\eta^{*}, \mathbf{b}$ is the long-run stable state and hence $\frac{1}{\tilde{\pi}_{\max }} \equiv \frac{1}{\tilde{\pi}(\tilde{b})} \leq K_{\pi}$, and

$$
\frac{1}{\tilde{\pi}_{\min }} \equiv \frac{1}{\tilde{\pi}(\tilde{a})} \leq K_{\pi} \exp \{\varphi(\tilde{\mathbf{a}})\}
$$

where

$$
\begin{aligned}
\varphi(\tilde{\mathbf{a}}) & =\left(N\left(\beta-\ln 2^{-1}\right)-\ln \left(1-\lambda_{2}\left(G_{2, l}\right)\right)\right)-\left(2 l(2 l+1)\left(\beta-\ln 2^{-1}\right)+N F\left(\frac{2 l(2 l+1)}{N}\right)\right) \\
& =(N-2 l(2 l+1))\left(\beta-\ln 2^{-1}\right)-N F\left(\frac{2 l(2 l+1)}{N}\right)-\ln \left(1-\lambda_{2}\left(G_{2, l}\right)\right)
\end{aligned}
$$

Substituting for $\frac{1}{\tilde{\pi}_{\min }}$ and $\frac{1}{\tilde{\pi}_{\min }}$ as in the proof of Proposition 8 yields the desired result. 
The $n$-dimensions $l$-max distance interactions networks present a good example of effect of progressively increasing information sets of players. That is starting from players observing only actions of the other players one step away, then two steps away, and so forth. As $l$ (information set) increases the expected waiting time to the long-run stable state increases, but the convergence time decreases. As $l$ increases the network and hence the convergence rates tend to that of global interactions. It is easy to show the convergence time for global interactions when $\eta_{a b}<\eta_{b a}$ to be

$$
T_{c} \leq \kappa_{1}\left(\kappa_{2}+\left(1-2 \eta_{a b}\right)(N-1)\left(\beta-\ln 2^{-1}\right)\right) \exp \left\{N\left(\eta_{b a}\left(\beta-\ln 2^{-1}\right)+F\left(\eta_{b a}\right)\right)\right\},
$$

which by comparison is less than the convergence time for 2-dimensions l-max distance interactions in (4.5.14) whenever $l<\frac{1}{2} \sqrt{N}$. In fact, the convergence time for global interactions in (4.5.17) is smaller than that for $n$-dimensions nearest neighbor interactions network (cf. in Proposition 8) as well. The general result is then that the convergence time for under global interactions is less than that under local interactions whenever global contagion is feasible.

\subsection{RANDOM NETWORKS}

Most real world networks assume complex structures. Several authors have proposed various ways to capture the properties of such networks e.g. clustering coefficient and degree distribution. For our purpose, the degree distribution is the most suitable for capturing the effect of network topology on the convergence rates. The degree distribution $P=\{p(k)\}_{k \geq 0}$ describes the number of agents in the population with a given degree. We denote by $\langle k\rangle$ for average degree. The following definitions related to the degree distribution are useful for the results that follow.

Definition 10: Let $G$ and $G^{\prime}$ be two networks with respective degree distributions $P$ and $P^{\prime}$.

(i) A degree distribution $P$ is said to first order stochastically dominate (FOSD) $P^{\prime}$ if $\sum_{k=0}^{K} p(k) \leq \sum_{k=0}^{K} p^{\prime}(k)$ for $1 \leq K \leq \infty$. Or for any non-decreasing function $f$ : $\mathbb{R} \rightarrow \mathbb{R}$

$$
\sum_{k=0}^{\infty} f(k) p^{\prime}(k) \leq \sum_{k=0}^{\infty} f(k) p(k) .
$$

(ii) A degree distribution $P$ is said to second order stochastically dominate (SOSD) $P^{\prime}$ if for any non-decreasing concave function $f: \mathbb{R} \rightarrow \mathbb{R}, \sum_{k=0}^{\infty} f(k) p^{\prime}(k) \leq \sum_{k=0}^{\infty} f(k) p(k)$.

(ii) A degree distribution $P$ is said to be a mean preserving spread (MPS) of $P^{\prime}$ if $P^{\prime} S O S D$ $P$ and that both have the same mean. 
The following proposition provides the relationship between degree distributions and convergence rates.

Proposition 10: Let $G$ and $G$ be two networks with respective degree distributions $P$ and $P^{\prime}$ and let the underlying game be $2 \times 2$ matrix game of Table 12 with $\eta_{a b}<\frac{1}{2}$. We write $\mathbb{E}_{G}$ for the expected value under network topology $G$ and $T_{\mathcal{C}}(G)$ for convergence time under G

(i) if P FOSD (or SOSD) $P^{\prime}$, then $\mathbb{E}_{G}\left[T(\mathbf{b}) \mid \mathbf{x}_{0}=\mathbf{a}\right] \geq \mathbb{E}_{G^{\prime}}\left[T(\mathbf{b}) \mid \mathbf{x}_{0}=\mathbf{a}\right]$

(ii) if $P$ MPS $P^{\prime}$ or $P^{\prime}$ FOSD $P$, then $T_{\mathcal{C}}(G) \leq T_{\mathcal{C}}\left(G^{\prime}\right)$

Proof. (i). For a given value of $\eta_{a b}<\frac{1}{2}$, the quantity $r(\mathbf{a}, \partial \mathbf{b})$ is an increasing function of network connectivity. This is clearly evidenced in the analysis of deterministic networks of Section 4.5. For example under 1-dimensions nearest neighbor interactions network $r(\mathbf{a}, \partial \mathbf{b})=\frac{1}{N}$ while under global interactions $r(\mathbf{a}, \partial \mathbf{b}) \approx \eta_{a b}$. We thus write $r(\mathbf{a}, \partial \mathbf{b}):=f(\langle k\rangle)$, where $f$ is an non-decreasing function in $\langle k\rangle$. Since $P$ FOSD (or SOSD) $P^{\prime}$ implies that $\langle k\rangle \geq\left\langle k^{\prime}\right\rangle$, it follows that $r_{G}(\mathbf{a}, \partial \mathbf{b})$ under $G$ is greater than $r_{G^{\prime}}(\mathbf{a}, \partial \mathbf{b})$ under $G^{\prime}$. Since $\mathscr{P}$ is identical in both cases, $\mathbb{E}_{G}\left[T(\mathbf{b}) \mid \mathbf{x}_{0}=\mathbf{a}\right] \geq$ $\mathbb{E}_{G^{\prime}}\left[T(\mathbf{b}) \mid \mathbf{x}_{0}=\mathbf{a}\right]$.

(ii). When $\eta_{a b}<\frac{1}{2}$, all players for whom $\left\lceil\eta_{b a} k_{i}\right\rceil \geq k_{i}$ require all their neighbors to switch to $a$ for them to do likewise. Denote by $\mathcal{N}_{b a}(G)$ for the subset of players for whom $\left\lceil\eta_{b a} k_{i}\right\rceil \geq k_{i}$ given the network $G$. That is

$$
\mathcal{N}_{b a}(G)=\left\{i \in \mathcal{N}:\left\lceil\eta_{b a} k_{i}\right\rceil \geq k_{i}\right\}
$$

Let $\bar{k}_{a}$ be an integer such that all $i$ for whom $k_{i} \leq \bar{k}_{a}$ belong to $\mathcal{N}_{b a}(G)$. Write $N_{b a}(G)$ for the cardinality of $\mathcal{N}_{b a}(G)$, that is $N_{b a}(G)=N \sum_{k=0}^{\bar{k}_{a}} p(k)$. For a given network, hence $\bar{k}_{a}, N_{b a}(G)$ is a non-increasing function of average degree $\langle k\rangle$. That is $N_{b a}(G):=f(\langle k\rangle)$ where $f$ is non-increasing in $\langle k\rangle$. For $P$ FOSD (or SOSD) $P^{\prime}$, $N_{b a}(G) \leq N_{b a}\left(G^{\prime}\right)$, and since $r_{G}(\mathbf{b}, \partial \mathbf{a})$ is an increasing function of $N_{b a}(G)$ then

$$
r_{G}(\mathbf{b}, \partial \mathbf{a}) \leq r_{G^{\prime}}(\mathbf{b}, \partial \mathbf{a})
$$

It then follows that for a given $\mathscr{P}$ hence $\beta, \mathbb{E}_{G}\left[T(\mathbf{a}) \mid \mathbf{x}_{0}=\mathbf{b}\right] \leq \mathbb{E}_{G^{\prime}}\left[T(\mathbf{a}) \mid \mathbf{x}_{0}=\mathbf{b}\right]$. Note also that for a given network say $G, \mathbb{E}_{G}\left[T(\mathbf{a}) \mid \mathbf{x}_{0}=\mathbf{b}\right] \geq \mathbb{E}_{G}\left[T(\mathbf{b}) \mid \mathbf{x}_{0}=\mathbf{a}\right]$ whenever $\eta_{a b}<\frac{1}{2}$. Such that the maximum expected waiting time for $G$ and $G^{\prime}$ are $\mathbb{E}_{G}\left[T(\mathbf{a}) \mid \mathbf{x}_{0}=\mathbf{b}\right]$ and $\mathbb{E}_{G^{\prime}}\left[T(\mathbf{a}) \mid \mathbf{x}_{0}=\mathbf{b}\right]$ respectively. Consequently $\mathscr{T}_{G} \leq \mathscr{T}_{G^{\prime}}$.

We then need to establish the relationships for the minimum and maximum values of the stationary distributions. First note that since $\tilde{\mathbf{b}}$ is the long-run stable subset 
and hence $\mathbf{b}$ is the long-run stable state, $\frac{1}{\tilde{\pi}_{\max }} \equiv \frac{1}{\tilde{\pi}(\tilde{b})} \leq K_{\pi}$ for both networks. We then have that for each network,

$$
\frac{1}{\tilde{\pi}_{\min }} \equiv \frac{1}{\tilde{\pi}(\tilde{a})} \leq K_{\pi} \exp \left\{\varphi_{G}(\tilde{\mathbf{a}})\right\}
$$

where

$$
\begin{aligned}
\varphi_{G}(\tilde{\mathbf{a}}) & =\left[N c_{G}(\mathbf{b}, \partial \mathbf{a})+N F\left(r_{G}(\mathbf{b}, \partial \mathbf{a})\right)-\ln \left(1-\lambda_{2}(G)\right)\right]-\left[N c_{G}(\mathbf{a}, \partial \mathbf{b})+N F\left(r_{G}(\mathbf{a}, \partial \mathbf{b})\right)\right. \\
& \left.-K_{\mathbf{a}} \ln \left(1-\lambda_{2}(G)\right)\right] \\
& =N\left[r_{G}(\mathbf{b}, \partial \mathbf{a})-r_{G}(\mathbf{a}, \partial \mathbf{b})\right]\left(\beta-\ln 2^{-1}\right)+N\left[F\left(r_{G}(\mathbf{b}, \partial \mathbf{a})\right)-F\left(r_{G}(\mathbf{a}, \partial \mathbf{b})\right)\right] \\
& -\left[\left(1-K_{\mathbf{a}}\right) \ln \left(1-\lambda_{2}(G)\right)\right]
\end{aligned}
$$

where we have again taken $K_{\mathbf{b}}=1$, and $K_{\mathbf{a}}<1$. Similarly,

$$
\begin{aligned}
\varphi_{G^{\prime}}(\tilde{\mathbf{a}}) & =N\left[r_{G^{\prime}}(\mathbf{b}, \partial \mathbf{a})-r_{G^{\prime}}(\mathbf{a}, \partial \mathbf{b})\right]\left(\beta-\ln 2^{-1}\right)+N\left[F\left(r_{G^{\prime}}(\mathbf{b}, \partial \mathbf{a})\right)-F\left(r_{G^{\prime}}(\mathbf{a}, \partial \mathbf{b})\right)\right] \\
& -\left[\left(1-K_{\mathbf{a}}\right) \ln \left(1-\lambda_{2}\left(G^{\prime}\right)\right)\right]
\end{aligned}
$$

Since $r_{G}(\mathbf{b}, \partial \mathbf{a}) \leq r_{G^{\prime}}(\mathbf{b}, \partial \mathbf{a})$ and $r_{G}(\mathbf{a}, \partial \mathbf{b}) \geq r_{G^{\prime}}(\mathbf{a}, \partial \mathbf{b})$, it follows that

$$
r_{G}(\mathbf{b}, \partial \mathbf{a})-r_{G}(\mathbf{a}, \partial \mathbf{b}) \leq r_{G^{\prime}}(\mathbf{b}, \partial \mathbf{a})-r_{G^{\prime}}(\mathbf{a}, \partial \mathbf{b})
$$

Similarly, since $P$ MPS $P^{\prime}$ or $P^{\prime}$ FOSD $P$ implies that $\left(1-\lambda_{2}\left(G^{\prime}\right)\right) \leq\left(1-\lambda_{2}(G)\right)$ and hence $-\ln \left(1-\lambda_{2}\left(G^{\prime}\right)\right) \geq-\ln \left(1-\lambda_{2}(G)\right)$. It then follows that $\varphi_{G}(\tilde{\mathbf{a}}) \leq \varphi_{G^{\prime}}(\tilde{\mathbf{a}})$, consequently $T_{\mathcal{C}}(G) \leq T_{\mathcal{C}}\left(G^{\prime}\right)$

The result in Proposition 10 supplements that for deterministic networks above. That is, the expected waiting times to the long-run stable set is shorter for sparsely connected than highly connected networks. Similarly, the convergence time is longer for sparsely connected than highly connected networks. The method of characterization is however complementary. Several authors have characterized the effect of network topologies on individual behavior and payoff in terms of degree distributions and using stochastic dominance relations (e.g Jackson and Yariv [55]). Proposition 10 provides similar characteristic analysis but for convergence rates, such that once the degree distributions of any two networks is known, then a comparative analysis of convergence rates can be performed. 


\subsection{STEP-BY-STEP EVOLUTION}

When global contagion is not feasible, that is when $\eta_{a b}>\eta^{*}$ for any pair of actions $a \neq b$, the network enhances the number of closed communication classes. Take for example the case of 2-dimensions nearest neighbor interactions network, if both $\eta_{a b}>\eta^{*}$ and $\eta_{b a}>\eta^{*}$ then global contagion is not feasible. Under this condition, if $\eta_{a b}<\eta_{b a}$ then all states in which one or more enclaves of four players all play $b$ become closed communication classes. To get from an all $a$ state to an all $b$ state, the chain goes through these intermediate closed communication classes induced by the network topology. It may seem at first that the presence of such intermediate states will lead to slow convergence rates when compared to making direct transition say from $\mathbf{a} \rightarrow \mathbf{b}$. On the contrary however, these intermediate states do speed-up evolution between closed communication classes induced by the payoff structure. This phenomenon was referred to as step-by-step evolution by Ellison [38]. Below, we use a specific example to illustrate the convergence rates in step-by-step evolution.

We consider a set of eight agents whose interactions are governed by the network topology in Figure 10, and playing a pure coordination game in Table 12. The network can be divided into two cohesive subgroups $g_{1}=\{1,2,3,4,5\}$ and $g_{2}=\{6,7,8,9,10\}$. A player $i$ belongs to a given cohesive subgroup if and only if at least half of $i$ 's interactions are with members of that subgroup. The contagion threshold for this network is $\frac{1}{3}$. That is if $\eta_{a b} \leq \frac{1}{3}$ then starting from $a$, global contagion to occurs when at least one player (from any of the subgroups) plays $b$ Hence for $\eta_{a b} \leq \frac{1}{3}, r(\mathbf{a}, \partial \mathbf{b})=\frac{1}{10}$. When $\frac{1}{3}<\eta_{a b}<\frac{1}{2}$, global contagion is no longer feasible, though local contagion (within cohesive subgroups) can occur. We use this situation to demonstrate the rates of convergence under step-by-step evolution. For $\frac{1}{3}<\eta_{a b}<\frac{1}{2}$, there are two additional closed communication classes to a and $\mathbf{b} ; \mathbf{a}_{1}$ and $\mathbf{a}_{2}$ in which all members of $g_{1}$ play $a$ while those in $g_{2}$ all play $b$ and vise versa for $\mathbf{a}_{2}$.

CoRollary 4: Let the underlying game be $2 \times 2$ matrix game of Table 12 with $\frac{1}{3}<\eta_{a b}<$ $\frac{1}{2}$. Let the updating rule be the uniform error probabilities, and the topology of interactions be that in Figure 10. Then

(i) the expected waiting times are;

$$
\begin{aligned}
& \mathbb{E}\left[T(\mathbf{b}) \mid \mathbf{x}_{0}=\mathbf{a}\right] \leq K_{T} \exp \{2 \beta-3.6\} \\
& \mathbb{E}\left[T(\mathbf{a}) \mid \mathbf{x}_{0}=\mathbf{b}\right] \leq K_{T} \exp \{4(\beta-1)\}
\end{aligned}
$$




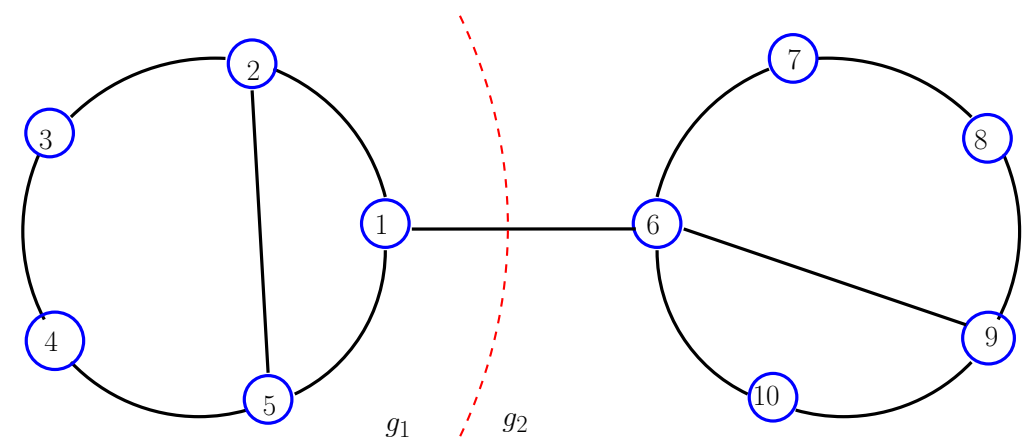

Figure 10: A general network with two cohesive subgroups

(ii) the convergence time is given by

$$
T_{C} \leq \kappa_{1}\left(\kappa_{2}+\varphi(\tilde{\mathbf{a}})\right) \exp \{4(\beta-1)\}
$$

where $\varphi(\tilde{\mathbf{a}})=4 \beta-0.8$.

Proof. For $\frac{1}{3}<\eta_{a b}<\frac{1}{2}$, the normalized radii are as follows: $r(\mathbf{a}, \partial \mathbf{b})=\frac{4}{10}$ (two players from $g_{1}$ and $g_{2}$ play $\left.b\right), r(\mathbf{b}, \partial \mathbf{a})=\frac{8}{10}$ (four players from $g_{1}$ and $g_{2}$ play $b$ ), $r\left(\mathbf{a}, \partial \mathbf{a}_{1}\right)=\frac{2}{10}=r\left(\mathbf{a}, \partial \mathbf{a}_{2}\right), r\left(\mathbf{b}, \partial \mathbf{a}_{1}\right)=\frac{4}{10}=r\left(\mathbf{b}, \partial \mathbf{a}_{2}\right), r\left(\mathbf{a}_{1}, \partial \mathbf{a}\right)=\frac{4}{10}=r\left(\mathbf{a}_{2}, \partial \mathbf{a}\right)$, $r\left(\mathbf{a}_{1}, \partial \mathbf{b}\right)=\frac{2}{10}, r\left(\mathbf{a}_{2}, \partial \mathbf{b}\right)=\frac{1}{10}, r\left(\mathbf{a}_{1}, \partial \mathbf{a}_{2}\right)=\frac{6}{10}$ and $r\left(\mathbf{a}_{2}, \partial \mathbf{a}_{1}\right)=\frac{5}{10}$. Figure 11 plots the quantity $c\left(\Omega, \partial \Omega^{\prime}\right)+F\left(r\left(\Omega, \partial \Omega^{\prime}\right)\right)$ for $\beta=5$.

(i). The expected waiting time from $\mathbf{a}$ to $\mathbf{b}$ is equivalent to letting $W=\left\{\mathbf{a}, \mathbf{a}_{1}, \mathbf{a}_{2}\right\}$ and $W^{c}=\{\mathbf{b}\}$, then computing $\mathbb{E}\left[T\left(W^{c}\right) \mid \mathbf{x}_{0}=\mathbf{a}\right]$. The least cost tree from a to $W^{c}$ is $\left\{\mathbf{a} \rightarrow \mathbf{a}_{2} \rightarrow \mathbf{b}, \mathbf{a}_{1} \rightarrow \mathbf{b}\right\}$ and $\left\{\mathbf{a}_{1} \rightarrow \mathbf{b}, \mathbf{a}_{2} \rightarrow \mathbf{b}\right\}$ minimizes $M_{W}(\mathbf{a})$. Such that $M_{w}$ and $M_{W}(\mathbf{a})$ are respectively

$$
\begin{gathered}
M_{W}=\left[c\left(\mathbf{a}, \partial \mathbf{a}_{2}\right)+F\left(r\left(\mathbf{a}, \partial \mathbf{a}_{2}\right)\right)\right]+\left[c\left(\mathbf{a}_{2}, \partial \mathbf{b}\right)+F\left(r\left(\mathbf{a}_{2}, \partial \mathbf{b}\right)\right)\right]+\left[c\left(\mathbf{a}_{1}, \partial \mathbf{b}\right)+F\left(r\left(\mathbf{a}_{1}, \partial \mathbf{b}\right)\right)\right] \\
M_{W}(\mathbf{a})=\left[c\left(\mathbf{a}_{1}, \partial \mathbf{b}\right)+F\left(r\left(\mathbf{a}_{1}, \partial \mathbf{b}\right)\right)\right]+\left[c\left(\mathbf{a}_{2}, \partial \mathbf{b}\right)+F\left(r\left(\mathbf{a}_{2}, \partial \mathbf{b}\right)\right)\right]
\end{gathered}
$$

Hence,

$$
H_{W}(\mathbf{a})=M_{W}-M_{W}(\mathbf{a})=c\left(\mathbf{a}, \partial \mathbf{a}_{2}\right)+F\left(r\left(\mathbf{a}, \partial \mathbf{a}_{2}\right)\right)=\frac{1}{5} \beta-0.36
$$




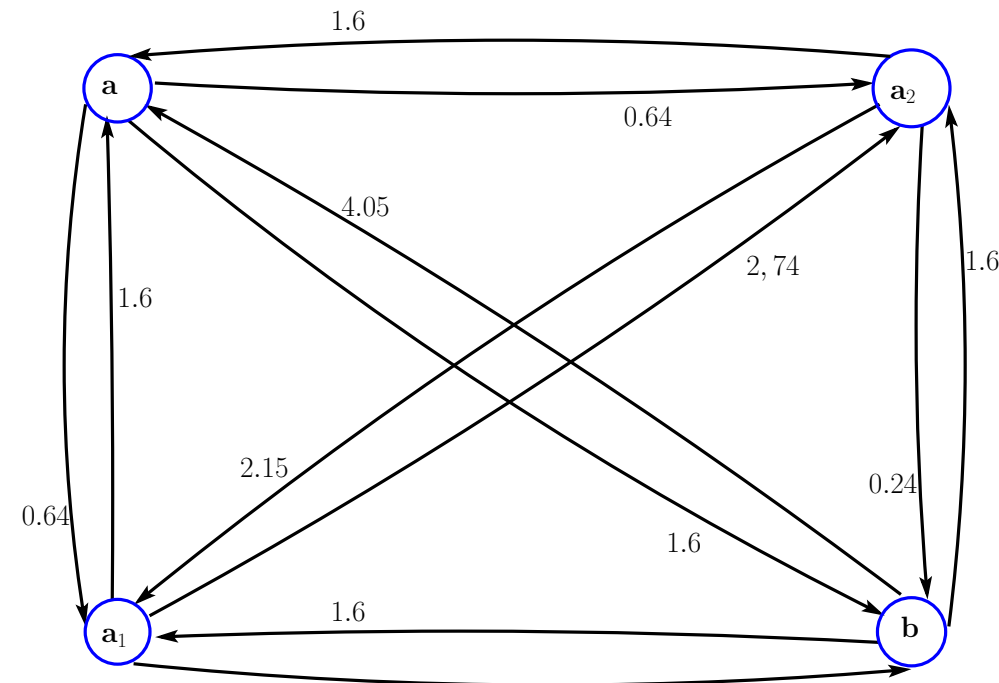

Figure 11: Each arc's weight is the quantity $c\left(\Omega, \partial \Omega^{\prime}\right)+F\left(r\left(\Omega, \partial \Omega^{\prime}\right)\right)$ for $\beta=5$

Implying that $\mathbb{E}\left[T\left(W^{c}\right) \mid \mathbf{x}_{0}=\mathbf{a}\right] \leq K_{T} \exp \left\{10\left(\frac{1}{5} \beta-0.36\right)\right\}=K_{T} \exp \{(2 \beta-3.6)\}$. Where the factor of 10 is the number of agents.

The expected waiting from $\mathbf{b}$ to $\mathbf{a}$ is equivalent to letting $W=\left\{\mathbf{b}, \mathbf{a}_{1}, \mathbf{a}_{2}\right\}$ and $W^{c}=\{\mathbf{a}\}$, then computing $\mathbb{E}\left[T\left(W^{c}\right) \mid \mathbf{x}_{0}=\mathbf{b}\right]$. The least cost tree from $\mathbf{b}$ to $W^{c}$ is $\left\{\mathbf{a}_{2} \rightarrow \mathbf{b} \rightarrow \mathbf{a}_{1} \rightarrow \mathbf{a}\right\}$ and $\left\{\mathbf{a}_{2} \rightarrow \mathbf{b} \rightarrow \mathbf{a}_{1}\right\}$ minimizes $M_{W}(\mathbf{b})$. Such that $M_{w}$ and $M_{W}(\mathbf{b})$ are respectively

$$
\begin{gathered}
M_{W}=\left[c\left(\mathbf{a}_{2}, \partial \mathbf{b}\right)+F\left(r\left(\mathbf{a}_{2}, \partial \mathbf{b}\right)\right)\right]+\left[c\left(\mathbf{b}, \partial \mathbf{a}_{1}\right)+F\left(r\left(\mathbf{b}, \partial \mathbf{a}_{1}\right)\right)\right]+\left[c\left(\mathbf{a}_{1}, \partial \mathbf{a}\right)+F\left(r\left(\mathbf{a}_{1}, \partial \mathbf{a}\right)\right)\right] \\
M_{W}(\mathbf{a})=\left[c\left(\mathbf{a}_{2}, \partial \mathbf{b}\right)+F\left(r\left(\mathbf{a}_{2}, \partial \mathbf{b}\right)\right)\right]+\left[c\left(\mathbf{b}, \partial \mathbf{a}_{1}\right)+F\left(r\left(\mathbf{b}, \partial \mathbf{a}_{1}\right)\right)\right]
\end{gathered}
$$

Hence,

$$
H_{W}(\mathbf{a})=M_{W}-M_{W}(\mathbf{a})=c\left(\mathbf{a}_{1}, \partial \mathbf{a}\right)+F\left(r\left(\mathbf{a}_{1}, \partial \mathbf{a}\right)\right)=\frac{2}{5} \beta-0.36
$$

Implying that $\mathbb{E}\left[T\left(W^{c}\right) \mid \mathbf{x}_{0}=\mathbf{b}\right] \leq K_{T} \exp \left\{10\left(\frac{2}{5} \beta-0.4\right)\right\}=K_{T} \exp \{4(\beta-1)\}$.

(ii). The maximum expected waiting time is for the transition from $\mathbf{b}$ to $\mathbf{a}$, hence $\mathscr{T} \equiv \mathbb{E}\left[T\left(W^{c}\right) \mid \mathbf{x}_{0}=\mathbf{b}\right] \leq K_{T} \exp \{4(\beta-1)\}$. Since $\mathbf{b}$ is the long-run stable state, then $\frac{1}{\tilde{\pi} \max } \equiv \frac{1}{\tilde{\pi}(\tilde{b})} \leq K_{\pi}$. Also, $\left\{\mathbf{a}_{2} \rightarrow \mathbf{b} \rightarrow \mathbf{a}_{1} \rightarrow \mathbf{a}\right\}$ is the least cost tree for $G(\{\mathbf{a})$ and $\left\{\mathbf{a} \rightarrow \mathbf{a}_{2} \rightarrow \mathbf{b}, \mathbf{a}_{1} \rightarrow \mathbf{b}\right\}$ for $G(\{\mathbf{b})$. Such that

$$
\frac{1}{\tilde{\pi}_{\min }} \equiv \frac{1}{\tilde{\pi}(\tilde{a})} \leq K_{\pi} \exp \left\{\varphi_{G}(\tilde{\mathbf{a}})\right\}
$$


where

$$
\begin{aligned}
\varphi_{G}(\tilde{\mathbf{a}}) & =10\left\{\left[c\left(\mathbf{a}_{2}, \partial \mathbf{b}\right)+F\left(r\left(\mathbf{a}_{2}, \partial \mathbf{b}\right)\right)\right]+\left[c\left(\mathbf{b}, \partial \mathbf{a}_{1}\right)+F\left(r\left(\mathbf{b}, \partial \mathbf{a}_{1}\right)\right)\right]+\left[c\left(\mathbf{a}_{1}, \partial \mathbf{a}\right)+F\left(r\left(\mathbf{a}_{1}, \partial \mathbf{a}\right)\right)\right]\right. \\
& \left.-\left[c\left(\mathbf{a}, \partial \mathbf{a}_{2}\right)+F\left(r\left(\mathbf{a}, \partial \mathbf{a}_{2}\right)\right)\right]+\left[c\left(\mathbf{a}_{2}, \partial \mathbf{b}\right)+F\left(r\left(\mathbf{a}_{2}, \partial \mathbf{b}\right)\right)\right]+\left[c\left(\mathbf{a}_{1}, \partial \mathbf{b}\right)+F\left(r\left(\mathbf{a}_{1}, \partial \mathbf{b}\right)\right)\right]\right\} \\
& =10\left\{\left[\frac{4}{5} \beta-0.8\right]-\left[\frac{2}{5} \beta-0.72\right]\right\} \\
& =10\left\{\frac{2}{5} \beta-0.08\right\}
\end{aligned}
$$

Corollary 4 underscores the role played by intermediate closed communication sets induced by the topology of the network in increasing convergence rates. If the chain is to be restricted to the direct transition from $\mathbf{a}$ to $\mathbf{b}$ rather than through intermediate closed communication sets, then the expected waiting time would be

$$
\mathbb{E}\left[T(\mathbf{b}) \mid \mathbf{x}_{0}=\mathbf{a}\right] \leq K_{T} \exp \left\{10\left(\frac{4}{10}\left(\beta-\ln 2^{-1}\right)+F\left(\frac{4}{10}\right)\right)\right\}=K_{T} \exp \{4(\beta-1)\}
$$

which is greater than the expected waiting time in (4.7.1) for the case with intermediate limit states. A similar argument follows for $\mathbb{E}\left[T(\mathbf{a}) \mid \mathbf{x}_{0}=\mathbf{b}\right]$.

In comparison to the case in which the 10 players interact globally, from $(4 \cdot 5 \cdot 17)$ we have

$$
T_{c} \leq \kappa_{1}\left(\kappa_{2}+9\left(1-2 \eta_{a b}\right)\left(\beta-\ln 2^{-1}\right)\right) \exp \left\{10\left(\eta_{b a}\left(\beta-\ln 2^{-1}\right)+F\left(\eta_{b a}\right)\right)\right\},
$$

Such that for $\frac{1}{3}<\eta_{a b}<\frac{1}{2}$, which is equivalent to $\frac{1}{2}<\eta_{b a}<\frac{2}{3}, T_{c}$ in (4.7.5) is greater than $T_{\mathcal{C}}$ in (4.7.3) for $\beta$ sufficiently large. Unlike in the case of local interactions in which global contagion is feasible above, for local interactions with step-by-step evolution the convergence time is shorter than under global interactions. Clearly, the presence of cohesive subgroups reduces the convergence time and this result generalizes to all networks with cohesive subgroups. The intuition behind the result is that the presence of intermediate closed communication classes reduces the resistance (sizes of basins of attraction) hence the minimum exit time for all closed communication classes involved, including the long-run stable set. This effectively reduces the expected waiting time between any pair of closed communication classes, hence the time it takes the chain to mix. 
This Chapter studies extensively the convergence rates for stochastic evolution in networks. We identify the payoff gains, noise level and network topology to be the key factors. Unlike previous analysis in the literature that focused on limit noise, we place emphasis on keeping the noise levels positive. Analysis that relies on limit noise fails to capture some aspects of evolutionary dynamics in networks. The network topology and payoff gains interactively determine whether or not an action is globally contagious. Globally contagious action are those with the highest payoff gains, hence are also those that are played in the long-run.

Several insightful results derive from our analysis. First, for given payoff gains, the more sparsely connected a network is the more likely that an action with the highest payoff gain is globally contagious. When global contagion is feasible, the expected waiting times from any other closed communication class to the long-run stable closed communication class are independent of the population size. For a given family of networks, global contagion also implies that the expected waiting time for highly connected network is shorter than for sparsely connected networks. Secondly, Whenever global contagion is feasible, sparsely connected networks tend to have longer convergence time than highly connected networks, longer than even the case of global interactions. Finally, if the payoff gains and network topology induce multiple intermediate closed communication classes, that is when global contagion is infeasible, then the convergence time is faster than both under global interactions and other interaction networks in which global contagion is feasible. Possible extensions to this Chapter include generalizations to other models of mistakes, beyond the uniform mistakes model we have considered.

\subsection{APPENDIX}

\subsubsection{Proof of Lemma 10}

Given that the chain is in some state $\mathbf{x}$ of $\Omega$, let $a$ be the action that should be played by at least $R\left(\Omega, \partial \Omega^{\prime}\right)$ players for the transition $\Omega \rightarrow \Omega$ to occur. If we let $I_{i}$ be a binary random variable taking on values one with probability $\mathscr{P}$ and zero otherwise, then

$$
P_{\mathcal{\varepsilon}}\left(\Omega, \partial \Omega^{\prime}\right)=\mathbb{P}\left(\sum_{i=1}^{N} I_{i}=R\left(\Omega, \partial \Omega^{\prime}\right)\right)
$$


The expression for the right hand side of (4.9.1) can be established using techniques for concentration bounds. From Binomial probabilities, we have (we write $R$ for the short form of $R\left(\Omega, \partial \Omega^{\prime}\right)$ )

$$
\mathbb{P}\left(\sum_{i=1}^{N} I_{i}=R\left(\Omega, \partial \Omega^{\prime}\right)\right)=\frac{N !}{R !(N-R) !} \mathscr{P}^{R}(1-\mathscr{P})^{N-R}
$$

Using Sterling's approximation we have

$$
\begin{aligned}
\frac{N !}{R !(N-R) !} & \approx \frac{N^{N} \sqrt{2 \pi N} e^{-N}}{R^{R} \sqrt{2 \pi R} e^{-R}(N-R)^{N-R} \sqrt{2 \pi(N-R)} e^{-(N-R)}} \\
& =\frac{N^{N}}{R^{R}(N-R)^{N-R}} \sqrt{\frac{N}{2 \pi R(N-R)}} \\
& =\sqrt{\frac{N}{2 \pi R(N-R)}} \exp (N \ln N-R \ln R-(N-R) \ln (N-R)) \\
& =\sqrt{\frac{1}{2 \pi r(1-r) N}} \exp \{-N(r \ln r-(1-r) \ln (1-r))\}
\end{aligned}
$$

We let $K_{N}=\frac{1}{\sqrt{2 \pi r(1-r) N}}$, and by rewriting

$$
\mathscr{P}^{R}(1-\mathscr{P})^{N-R}=\exp \{N(r \ln \mathscr{P}+(1-r) \ln (1-\mathscr{P}))\},
$$

we then have

$$
\begin{aligned}
P_{\varepsilon}\left(\Omega, \partial \Omega^{\prime}\right) & \approx K_{N} \exp \left\{N\left(r \ln \mathscr{P}-r \ln r+(1-r) \frac{\ln (1-\mathscr{P})}{\ln (1-r)}\right)\right\} \\
& \approx K_{N} \exp \{N(r \ln \mathscr{P}-r \ln r-(1-r) \ln (1-r))\} \\
& =K_{N} \exp \left\{-N\left(c\left(\Omega, \partial \Omega^{\prime}\right)+F\left(r\left(\Omega, \partial \Omega^{\prime}\right)\right)\right)\right\}
\end{aligned}
$$

where $c\left(\Omega, \partial \Omega^{\prime}\right)=-r\left(\Omega, \partial \Omega^{\prime}\right) \ln \mathscr{P}$ and $F(r)=r \ln r-(1-r) \ln (1-r)$.

\subsubsection{Proof of Theorem 3}

The proof of Theorem 3 follows from Lemma 9 and (4.3.6). Given the expressions for $P_{\mathcal{E}}\left(\Omega, \Omega^{\prime}\right)$

$$
P_{\mathcal{\varepsilon}}(g)=\prod_{\left(\Omega, \Omega^{\prime}\right) \in g} P_{\mathcal{\varepsilon}}\left(\Omega, \Omega^{\prime}\right) \approx K_{1} \exp \left\{\sum_{\left(\Omega, \Omega^{\prime}\right) \in g}\left(-N\left(c\left(\Omega, \partial \Omega^{\prime}\right)+F\left(r\left(\Omega, \partial \Omega^{\prime}\right)\right)\right)+\gamma\left(\Omega^{\prime}\right)\right)\right\}
$$


where $K_{1}=\left(K_{N}\right)^{\# g}$. For any $W \neq \varnothing$, any $\Omega \in W$,

$$
\begin{aligned}
\mathbb{E}\left[T\left(W^{c}\right) \mid \mathbf{x}_{0} \in \Omega\right] & =\left(\sum_{\Omega^{\prime} \in W} \sum_{g \in G_{\Omega, \Omega^{\prime}}\left(W \cup\left\{\Omega^{\prime}\right\}\right)} P_{\mathcal{\varepsilon}}(g)\right)\left(\sum_{g \in G(W)} P_{\mathcal{\varepsilon}}(g)\right)^{-1} \\
& \leq K_{T} \frac{\max _{\Omega \in W} \max _{g \in G_{\Omega, \Omega^{\prime}}\left(W \cup\left\{\Omega^{\prime}\right\}\right)} P_{\mathcal{\varepsilon}}(g)}{\min _{g \in G\left(W^{c}\right)} P_{\mathcal{\varepsilon}}(g)} \\
& =K_{T} \exp \left\{\min _{g \in G\left(W^{c}\right)} \psi(g)-\min _{\Omega^{\prime} \in W} \min _{g \in G_{\Omega, \Omega^{\prime}}\left(W \cup\left\{\Omega^{\prime}\right\}\right)} \psi(g)\right\}
\end{aligned}
$$

where $K_{T}$ is some constant;

$$
K_{T}=\frac{(\# W)\left(\# G_{\Omega, \Omega^{\prime}}\left(W \cup\left\{\Omega^{\prime}\right\}\right)\right)}{\# G\left(W^{c}\right)} .
$$

\subsubsection{Proof of Theorem 4}

The proof bounds mixing times of Markov chains appear in various version in the literature, see for example Levin et al. [70]. Here, we are interested in the properties of the reduced chain $\left(\tilde{\Omega}, \tilde{P}_{\mathcal{\varepsilon}}\right)$ with stationary distribution $\tilde{\pi}$. The formal definition of $\left(\tilde{\Omega}, \tilde{P}_{\varepsilon}\right)$ is as follows (a generalization of the collapsed Markov chain in Aldous and Fill [4, Chapter 2]):

$$
\begin{aligned}
\tilde{P}_{\mathcal{E}}(\mathbf{x}, \mathbf{y}) & =P_{\mathcal{\varepsilon}}(\mathbf{x}, \mathbf{y}) \\
\tilde{P}_{\mathcal{E}}(\mathbf{y}, \tilde{\Omega}) & =\sum_{\mathbf{x} \in \tilde{\Omega}} P_{\mathcal{E}}(\mathbf{y}, \mathbf{x}) \\
\tilde{P}_{\mathcal{E}}(\tilde{\Omega}, \mathbf{y}) & =\frac{1}{\pi(\tilde{\Omega})} \sum_{\mathbf{x} \in \tilde{\Omega}} \pi(\mathbf{x}) P_{\mathcal{E}}(\mathbf{x}, \mathbf{y}), \\
\tilde{P}_{\mathcal{E}}\left(\tilde{\Omega}, \tilde{\Omega}^{\prime}\right) & =\frac{1}{\pi(\tilde{\Omega})} \sum_{\mathbf{x} \in \tilde{\Omega}} \sum_{\mathbf{y} \in \tilde{\Omega}^{\prime}} \pi(\mathbf{x}) P_{\mathcal{\varepsilon}}(\mathbf{x}, \mathbf{y})
\end{aligned}
$$

The following lemma is an immediate consequence of the above definition of a collapsed Markov chain.

LeMMA 11: Let $\pi$ and $\tilde{\pi}$ be the stationary distributions of $\left(\mathbf{X}, \tilde{P}_{\mathcal{\varepsilon}}\right)$ and $\left(\tilde{\Omega}, \tilde{P}_{\mathcal{\varepsilon}}\right)$ respectively. Then for any $\tilde{\Omega} \in \tilde{\Omega}, \tilde{\pi}(\tilde{\Omega})=\pi(\tilde{\Omega})=\sum_{\mathbf{x} \in \tilde{\Omega}} \pi(\mathbf{x})$

Proof. Note that $\pi(\tilde{\Omega})=\sum_{\mathbf{x} \in \tilde{\Omega}} \pi(\mathbf{x})$. Let $\# \mathbf{X}$ and $\# \tilde{\Omega}$ be the cardinalities of $\mathbf{X}$ and $\tilde{\Omega}$ respectively. Define an event matrix $\mathcal{E}$ as an $\# \mathbf{X} \times \# \mathbf{\Omega}$ matrix whose entries take on a value one if a state $\mathbf{x}$ belongs to $\tilde{\Omega}$ and zero otherwise. Denote by $\mathcal{E}_{\tilde{\Omega}}$ for the $\tilde{\Omega}^{\text {th }}$ column of $\mathcal{E}$. It then follows that $\tilde{P}_{\mathcal{E}} \mathcal{E}=\mathcal{E} \tilde{P}_{\mathcal{E}}$, and that

$$
\pi(\tilde{\Omega})=\pi \mathcal{E}_{\tilde{\Omega}} \quad \forall \tilde{\Omega} \in \tilde{\Omega}
$$


Consequently, $\pi \mathcal{E}=\pi \tilde{P}_{\mathcal{E}} \mathcal{E}=\pi \mathcal{E} \tilde{P}_{\mathcal{E}}$. Implying that $\pi \mathcal{E}$ is the stationary distribution of $\tilde{P}_{\mathcal{E}}$, hence $\tilde{\pi}=\pi \mathcal{E}$.

Since $\tilde{P}_{\varepsilon}$ is not symmetric, the first step of the proof is to transform $\tilde{P}_{\varepsilon}$ into a symmetric matrix. We can then exploit spectral properties of symmetric matrices. From reversibility of $\tilde{P}_{\varepsilon}$ and hence $\tilde{P}_{\varepsilon}$, that is $\tilde{\pi}(\tilde{\Omega}) \tilde{P}_{\varepsilon}\left(\tilde{\Omega}, \tilde{\Omega}^{\prime}\right)=\tilde{\pi}\left(\tilde{\Omega}^{\prime}\right) \tilde{P}_{\varepsilon}\left(\tilde{\Omega}^{\prime}, \tilde{\Omega}\right)$

Recall that $\tilde{P}_{\varepsilon}$ is reversible. That is given the stationary distribution $\tilde{\pi}$,

$$
\tilde{\pi}(\tilde{\Omega}) \tilde{P}_{\varepsilon}\left(\tilde{\Omega}, \tilde{\Omega}^{\prime}\right)=\tilde{\pi}\left(\tilde{\Omega}^{\prime}\right) \tilde{P}_{\varepsilon}\left(\tilde{\Omega}^{\prime}, \tilde{\Omega}\right) \quad \forall \tilde{\Omega}, \tilde{\Omega}^{\prime} \in \mathbf{\Omega}
$$

We can define an equivalent symmetric matrix $S$ such that

$$
S\left(\tilde{\Omega}, \tilde{\Omega}^{\prime}\right)=\sqrt{\frac{\tilde{\pi}(\tilde{\Omega})}{\tilde{\pi}\left(\tilde{\Omega}^{\prime}\right)}} \tilde{P}_{\varepsilon}\left(\tilde{\Omega}, \tilde{\Omega}^{\prime}\right)
$$

The reason for introducing $S$ is to be able to exploit the properties of symmetric matrices, particularly the spectral theorem stating that, for symmetric matrices, there exists a set of orthonormal basis $\left\{\mathbf{u}_{i}\right\}_{i=1}^{|\Omega|}$, such that $\mathbf{u}_{i}$ is an eigenfunction corresponding to the real eigenvalue $\tilde{\mu}_{i}$.

Now, let us denote the diagonal matrix with elements $\tilde{\pi}(\tilde{\Omega})$ by $D$, then we have

$$
S=D^{\frac{1}{2}} \tilde{P}_{\varepsilon} D^{-\frac{1}{2}}
$$

Let $f_{i}=D^{-\frac{1}{2}} \mathbf{u}_{i}$, (where $\mathbf{u}_{i}{ }^{\prime}$ s are eigenfunctions of $S$ ) it follows that $f_{i}$ is an eigenfunction of $\tilde{P}_{\varepsilon}$ corresponding to eigenvalue $\tilde{\mu}_{i}$, and that $f_{i}$ 's are orthonormal with respect to $\tilde{\pi}$. That is

$$
\tilde{P}_{\varepsilon} f_{i}=\tilde{P}_{\varepsilon} D^{-\frac{1}{2}} \mathbf{u}_{i}=D^{-\frac{1}{2}}\left(D^{\frac{1}{2}} \tilde{P}_{\varepsilon} D^{-\frac{1}{2}}\right) \mathbf{u}_{i}=D^{-\frac{1}{2}} S \mathbf{u}_{i}=D^{-\frac{1}{2}} \tilde{\mu}_{i} \mathbf{u}_{i}=\tilde{\mu}_{i} f_{i} .
$$

To prove orthonormality of the functions $f_{i}$ 's with respect to $\tilde{\pi}$, its useful to take note of the following definition of the inner product.

Let $\langle.,$.$\rangle denote the inner product on \mathbb{R}^{\Omega}$, that is

$$
\langle f, g\rangle=\sum_{\tilde{\Omega} \in \Omega} f(\tilde{\Omega}) g(\tilde{\Omega}),
$$

then we can define the inner product with respect to the distribution $\tilde{\pi}$ as

$$
\langle f, g\rangle_{\tilde{\pi}}=\sum_{\tilde{\Omega} \in \Omega} f(\tilde{\Omega}) g(\tilde{\Omega}) \tilde{\pi}(\tilde{\Omega}) .
$$

Let $\delta_{i, j}$ denote the Dirac delta function (that is $\delta_{i, j}=1$ if and only if $i=j$ ), then

$$
\begin{aligned}
\delta_{i, j}=\left\langle\mathbf{u}_{i}, \mathbf{u}_{j}\right\rangle=\left\langle D^{\frac{1}{2}} f_{i}, D^{\frac{1}{2}} f_{j}\right\rangle=\sum_{\tilde{\Omega} \in \Omega} \tilde{\pi}(\tilde{\Omega})^{\frac{1}{2}} f(\tilde{\Omega}) \tilde{\pi}(\tilde{\Omega})^{\frac{1}{2}} g(\tilde{\Omega}) & =\sum_{\tilde{\Omega} \in \mathbf{\Omega}} f(\tilde{\Omega}) \tilde{\pi}(\tilde{\Omega}) g(\tilde{\Omega}) \\
& =\langle f, g\rangle_{\tilde{\pi}} .
\end{aligned}
$$


Note that $\tilde{P}_{\varepsilon}^{t}\left(\tilde{\Omega}, \tilde{\Omega}^{\prime}\right)$ is the $\tilde{\Omega}, \tilde{\Omega}^{\prime}$ element of $\tilde{P}_{\varepsilon}^{t}$, implying that $\tilde{P}_{\varepsilon}^{t}\left(\tilde{\Omega}, \tilde{\Omega}^{\prime}\right)=\left(\tilde{P}_{\varepsilon}^{t} \delta_{\tilde{\Omega}^{\prime}}\right)(\tilde{\Omega})$; where $\delta_{\tilde{\Omega}^{\prime}}(\tilde{\Omega})$ is a Dirac function assuming the value of unity for $\tilde{\Omega}=\tilde{\Omega}^{\prime}$ and zero otherwise. Notice also that $\delta_{\tilde{\Omega}^{\prime}}$ belongs to the inner product space $\mathbb{V}=\left(\mathbb{R}^{\Omega},\langle. . .\rangle_{\tilde{\pi}}\right)$, and since the set $\left\{f_{1}, \cdots, f_{|\Omega|}\right\}$ is an orthonormal basis of $\mathbb{V}$, then $\delta_{\tilde{\Omega}^{\prime}}$ can be written via basis decomposition as

$$
\delta_{\tilde{\Omega}^{\prime}}=\sum_{i=1}^{\eta}\left\langle\delta_{\tilde{\Omega}^{\prime}}, f_{i}\right\rangle_{\tilde{\pi}} f_{i}=\sum_{i=1}^{\eta} f_{i}\left(\tilde{\Omega}^{\prime}\right) \tilde{\pi}\left(\tilde{\Omega}^{\prime}\right) f_{i}
$$

Substituting (4.9.12) and $\tilde{P}_{\varepsilon}^{t} f_{i}=\tilde{\mu}_{i}^{t} f_{i}$ gives $\tilde{P}_{\varepsilon}^{t}\left(\tilde{\Omega}, \tilde{\Omega}^{\prime}\right)$ as

$$
\tilde{P}_{\varepsilon}^{t}\left(\tilde{\Omega}, \tilde{\Omega}^{\prime}\right)=\sum_{i=1}^{\eta} f_{i}\left(\tilde{\Omega}^{\prime}\right) \tilde{\pi}\left(\tilde{\Omega}^{\prime}\right) \tilde{\mu}_{i}^{t} f_{i}(\tilde{\Omega})=\tilde{\pi}\left(\tilde{\Omega}^{\prime}\right)+\sum_{i=2}^{\eta} f_{i}\left(\tilde{\Omega}^{\prime}\right) \tilde{\pi}\left(\tilde{\Omega}^{\prime}\right) \tilde{\mu}_{i}^{t} f_{i}(\tilde{\Omega})
$$

Taking the absolute values of (4.9.13) yields

$$
\begin{aligned}
\left|\tilde{P}_{\varepsilon}^{t}\left(\tilde{\Omega}, \tilde{\Omega}^{\prime}\right)-\tilde{\pi}\left(\tilde{\Omega}^{\prime}\right)\right| & =\sum_{i=2}^{\eta}\left|f_{i}\left(\tilde{\Omega}^{\prime}\right) \tilde{\pi}\left(\tilde{\Omega}^{\prime}\right) f_{i}(\tilde{\Omega}) \tilde{\mu}_{i}^{t}\right| \leq \sum_{i=2}^{\eta}\left|f_{i}\left(\tilde{\Omega}^{\prime}\right) \tilde{\pi}\left(\tilde{\Omega}^{\prime}\right) f_{i}(\tilde{\Omega})\right|\left|\tilde{\mu}_{2}\right|^{t} \\
& \leq \tilde{\pi}\left(\tilde{\Omega}^{\prime}\right)\left[\sum_{i=2}^{\eta} f_{i}^{2}\left(\tilde{\Omega}^{\prime}\right) \sum_{i=2}^{\eta} f_{i}^{2}(\tilde{\Omega})\right]^{\frac{1}{2}}\left|\tilde{\mu}_{2}\right|^{t}
\end{aligned}
$$

where the last inequality follows from Cauchy-Schwarz inequality. Following from the definition of inner product together with that of $\delta_{\tilde{\Omega}^{\prime}}(\tilde{\Omega})$ in (4.9.12), and relying on the orthonormality of the set $f_{i}$, we have

$$
\tilde{\pi}\left(\tilde{\Omega}^{\prime}\right)=\left\langle\delta_{\tilde{\Omega}^{\prime}}, \delta_{\tilde{\Omega}^{\prime}}\right\rangle=\left\langle\sum_{i=1}^{\eta} f_{i}\left(\tilde{\Omega}^{\prime}\right) \tilde{\pi}\left(\tilde{\Omega}^{\prime}\right) f_{i}, \sum_{i=1}^{\eta} f_{i}\left(\tilde{\Omega}^{\prime}\right) \tilde{\pi}\left(\tilde{\Omega}^{\prime}\right) f_{i}\right\rangle=\tilde{\pi}\left(\tilde{\Omega}^{\prime}\right)^{2} \sum_{i=1}^{\eta} f_{i}\left(\tilde{\Omega}^{\prime}\right)^{2}
$$

The implication is that $\sum_{i=2}^{\eta} f_{i}\left(\tilde{\Omega}^{\prime}\right)^{2} \leq \frac{1}{\tilde{\pi}\left(\tilde{\Omega}^{\prime}\right)}$, and consequently

$$
\left|\tilde{P}_{\varepsilon}^{t}\left(\tilde{\Omega}, \tilde{\Omega}^{\prime}\right)-\tilde{\pi}\left(\tilde{\Omega}^{\prime}\right)\right| \leq \frac{\tilde{\pi}\left(\tilde{\Omega}^{\prime}\right)}{\sqrt{\tilde{\pi}(\tilde{\Omega}) \tilde{\pi}\left(\tilde{\Omega}^{\prime}\right)}}\left|\tilde{\mu}_{2}\right|^{t}
$$

Such that if $\tilde{\Omega}_{0}$ is the initial state of $\left(\tilde{\Omega}, \tilde{P}_{\varepsilon}\right)$ with respective stationary distribution of $\tilde{\pi}\left(\tilde{\Omega}_{0}\right)$, then

$$
|| \mathbf{s}_{0} \tilde{P}_{\varepsilon}^{t}-\tilde{\pi}||=\frac{1}{2} \sum_{\tilde{\Omega}^{\prime} \in \Omega}\left|\tilde{P}_{\varepsilon}^{t}\left(\tilde{\Omega}_{0}, \tilde{\Omega}^{\prime}\right)-\tilde{\pi}\left(\tilde{\Omega}^{\prime}\right)\right| \leq \frac{1}{2} \sum_{\tilde{\Omega}^{\prime} \in \Omega} \frac{\tilde{\pi}\left(\tilde{\Omega}^{\prime}\right)}{\sqrt{\tilde{\pi}\left(\tilde{\Omega}_{0}\right) \tilde{\pi}\left(\tilde{\Omega}^{\prime}\right)}}\left|\tilde{\mu}_{2}\right|^{t} \leq \frac{1}{2} \frac{1}{\tilde{\pi}_{\min }}\left|\tilde{\mu}_{2}\right|^{t}
$$

where the last inequality follows from the fact that $\tilde{\pi}\left(\tilde{\Omega}_{0}\right) \tilde{\pi}\left(\tilde{\Omega}^{\prime}\right) \leq \min _{\tilde{\Omega} \in \tilde{\Omega}} \tilde{\pi}(\tilde{\Omega})^{2}=$ $\tilde{\pi}_{\min }$. 
104 CONVERGENCE RATES FOR STOCHASTIC EVOLUTION IN NETWORKS

For || $\mathbf{s}_{0} \tilde{P}_{\varepsilon}^{t}-\tilde{\pi}|| \leq \omega$, then $\frac{1}{2} \frac{1}{\tilde{\pi}_{\min }}\left|\tilde{\mu}_{2}\right|^{t} \leq \boldsymbol{\omega}$. Consequently, the convergence time is

$$
T_{c} \leq \frac{\ln \left(2 \omega \tilde{\pi}_{\min }\right)}{\ln \left|\tilde{\mu}_{2}\right|}
$$




\title{
BELIEFS DYNAMICS IN COMMUNICATION NETWORKS ${ }^{1}$
}

\begin{abstract}
Aвstract: We study the dynamics of individual beliefs and information aggregation when agents communicate via a social network. We provide a general framework of word-of-mouth communication and learning that captures the interactive effects of three main factors on the structure of individual beliefs resulting from such a dynamic process; that is historical factors-prior beliefs, learning mechanisms-rational and bounded-rational learning, and the topology of communication structure governing information exchange. More specifically, we provide conditions under which heterogeneity and consensus prevail. We then establish conditions on the structures of the communication network, prior beliefs and private information for public beliefs to correctly aggregate decentralized information. The speed of learning is also established, but most importantly, its implications on efficient information aggregation.
\end{abstract}

\subsection{INTRODUCTION}

Individual beliefs play a significant role in determining public opinions and decisions made under uncertainty, both of which in turn shape social welfare. For example the level of heterogeneity in beliefs about government policies such as public health and social integration initiatives affects their implementation. Decision making under uncertainty is a ubiquitous problem in economics and social settings. Examples include consumer decisions on a brand choice; adoption of agricultural products and information technologies; investment as well as legislative decisions among others. Our goal in this Chapter is to provide a comprehensive yet fundamental framework for characterizing the evolution of individual beliefs through word-of-mouth communication, and to establish conditions under which the resulting public beliefs correctly and efficiently aggregate decentralized information.

1 This Chapter has been published in UNU-MERIT working paper series: Opolot and Azomahou [9o] 
There are mainly three factors that influence the evolution of individual beliefs through word-of-mouth communication; historical factors-prior beliefs, the learning mechanism (the manner in which individuals incorporate new information into their beliefs)-rational or bounded-rational learning, and the topology of communication structure governing information exchange. To this we construct a comprehensive theoretical model that is fundamental enough to capture the main properties and general enough to enable us compare and contrast the properties of beliefs resulting from rational and bounded-rational learning. There exists a true state of nature unknown to all agents about which they update their beliefs. Agents possess heterogeneous prior beliefs that are not necessarily correlated to the true state of nature. Each agent receives private information that is informative about the true state of nature, and in turn updates their prior belief. The resulting private beliefs are then simultaneously communicated (or simply announced) to the immediate neighbors. ${ }^{2}$ After observing the neighbors' announcements, each agent incorporates the communicated beliefs into their private beliefs either by deducing the associated private signal in the case of rational learning, or by taking the weighted average of the announcements in the case of bounded-rational learning. The communication and learning process continues until none of the agents has new information to learn from the announcements of his neighbors, in which case their beliefs become "local public information". To differentiate private beliefs from beliefs that result at the end of the learning process, we refer to the later as public beliefs since they become public information at least to the first-order neighbors.

Unlike previous studies that focused on each of the three factors separately, the generalized framework we provide directly establishes an explicit characterization of how the three factors interactively shape individual beliefs. We also provide a stylized model for an exit game in which economic agents partially rely on their level of confidence in their beliefs in deciding whether or not to take an action (e.g. investing in a given project) or wait to collect more information. The waiting process is however costly, such each agent has to choose the optimal waiting time to taking an action. The purpose of this stylized model is to provide a characterization of the effect of the learning mechanisms and the topology of the communication structure on the efficiency of information aggregation. We start by making generalizations of some of the existing results in the literature. More specifically, we establish the structure of public beliefs under rational learning when agents are either certain or uncertain about others' prior beliefs. We then extend existing results on boundedrational learning to dynamic communication networks. The main contribution of

2 A private belief is what results after an agent incorporates his private information into his prior belief. 
the Chapter is the theorems providing the conditions on prior beliefs, private information and communication network structure for public beliefs to correctly and efficiently aggregate decentralized information. Public beliefs are said to be correct or to correctly aggregate private information if they fully incorporate private information of all agents, and asymptotically correct if in the limit of the population size, they converge in probability to the true state of nature. To compare the efficiency of information aggregation, we construct a simple decision problem in which agent's utility is the expected loss from mismatch between their actions and the true state of nature.

\subsubsection{Summary of results}

We now provide a summary of our findings. Proposition 5 provides the expressions for the structure of public beliefs under rational learning for finite population. When the communication network is common knowledge and connected, then a consensus in public beliefs arises only if the prior beliefs are identical and observable to the neighbors. It is not necessary for prior beliefs to be common knowledge among all agents for a consensus to emerge provided they are observable to the neighbors. Proposition 5 also shows that when the communication network is complete, a consensus in public beliefs obtains under uncertainty of prior beliefs provided the realized prior beliefs are identical and correlated. Heterogeneity in public beliefs arises under two cases. The first case is when the realized prior beliefs of neighbors are observable but heterogeneous, and the second case is when the realized prior beliefs are identical but not observable to the neighbors.

In Proposition 11, we generalize the bounded-rational learning models to dynamic communication networks. We show that provided the switching strategy is such that there exists a positive probability of realizing a connected network, then a consensus will obtain in a long-run. 3 Public beliefs will be heterogeneous otherwise.

In Theorems 6 and 7, we establish conditions for public beliefs to be asymptotically correct. That is conditions under which public beliefs converge in probability for a large population size to the true state of nature. Under rational learning, public beliefs will be asymptotically correct even when agents are uncertain of others' prior beliefs provided the prior beliefs and signals are independently distributed and of finite spaces. The topology of the communication network does not affect correctness of asymptotic public beliefs provided that it is common knowledge and connected.

3 A switching strategy is a function that maps an agent's current position in the network (or simply the current neighborhood) to another position in the next period. 
Contrary to rational learning, under bounded-rational learning, the topology of the communication network plays a significant role in determining correctness of public beliefs. We show that the network must be perfectly balanced and asymptotically balanced for public beliefs and asymptotic public beliefs respectively to be correct. We then characterize classes of networks that satisfy perfect and asymptotic balancedness conditions. In addition to restrictions on the network topology, we also find that under bounded-rational learning, public beliefs are asymptotically correct if and only if the prior beliefs are correlated to the true state of nature. Specifically, agents prior beliefs must be normally distributed with mean equal to the true state of nature and with finite variance.

In section 5.5 we compare the efficiency of private information aggregation by public beliefs under rational and bounded-rational learning. We show that the price of rationality, which we define as the ratio of expected social welfare under rational learning to the expected social welfare under bounded-rational learning, is an inverse function of the population size. Implying that the larger the population, the higher is the relative benefit of having an economy made up of rational agents. We show that the speed at which private information is aggregated is faster under rational learning than under bounded-rational learning.

\subsubsection{Relation to the literature}

This Chapter contributes to the existing literature in several ways. First, it is closely related to the literature on knowledge and consensus (e.g Geanakoplos and Polemarchakis [47], Parikh and Krasucki [92] and Krasucki [62]). Geanakoplos and Polemarchakis [47] showed that repeated communication of posterior beliefs between two agents who start with a common prior will eventually lead to a consensus in their public beliefs. Parikh and Krasucki [92] generalize the framework of Geanakoplos and Polemarchakis [47] to the case in which agents communicate a general family of functions that map information sets to messages, of which posterior beliefs are a special case. They then establish conditions on such functions under which a consensus in public beliefs obtains. Krasucki [62] extends the framework of Parikh and Krasucki [92] to multiple agents who communicate sequentially through a protocol that determines the sender and receiver. They show that if the communication protocol does not consist of cycles, then a consensus in public beliefs obtains. ${ }^{4}$ In all the above models, communication is sequential. That is it is defined by a protocol that selects

4 A cycle is a closed path, where a path from agent $i$ to agent $j$ is a connected set of links starting from $i$ and ending in $j$. 
a pair of agents (a sender and a receiver) and in each period only one of them is active. This is contrary to the case here in which agents act simultaneously. The second general difference is that in the above papers, private information is represented by an information set which is defined by a partition of the state space. Such representation of private information typically leads to multidimensional information structure, which implies that communication can take multiple rounds even in the case of two agents before a consensus in beliefs is finally reached. On the contrary we adopt a simpler information structure that allows us to focus on the main questions concerning learning in general networks and properties of public beliefs. Indeed, as in Geanakoplos and Polemarchakis [47] and Parikh and Krasucki [92] we find that when the communication network is complete or rather when posterior beliefs announcements are public, a consensus emerges provided prior beliefs are common knowledge and identical. But as an extension to this result, we show that it is not necessary for agents to be certain of others' prior beliefs for a consensus to obtain. All that matters is that prior beliefs are correlated and that the realizations are identical. When the communication network is not complete, we find as in Krasucki [62] that the network must be connected if a consensus is to obtain. We then characterize conditions for heterogeneity and correctness in public beliefs to obtain.

Secondly, this Chapter is related to the literature on Bayesian learning with rational agents in social networks (e.g Gale and Kariv [46], Rosenberg et al. [95] and Mueller-Frank [8o]). Just as we do in this here, these papers also consider simultaneous communication among agents but with the difference that agents communicate their actions rather than the posterior beliefs. 5 These papers also consider a multidimensional information structure as in the case of the models on knowledge and consensus above. The primary focus of this literature is on the uniformity and local indifference in the actions chosen by the agents at the end of the learning process, which is contrary to our focus on public beliefs. Nevertheless, some of our findings extend directly to situations where agents communicate actions rather than posterior beliefs. ${ }^{6}$ Rosenberg et al. [95] and Mueller-Frank [80] show that under the assumptions of connected network, common prior, common knowledge of strategies and network topology, heterogeneity in agents' actions at the end of learning process

5 There also exists a literature on sequential Bayesian learning in which agents make a decision once in a lifetime in an exogenously predefined order. When it is an agent's turn to act, he observes the history of actions of all agents that acted before him. The primary concern of this literature is establishing conditions under which informational cascades and herds behavior occurs. The main contributions are Banerjee [13], Bikhchandani et al. [16], Smith and Sorensen [109] and Acemoglu et al. [2].

6 This is particularly true because communicating posterior beliefs is equivalent to communicating actions when the action space is rich (for example the continuous action space) and/or when strategies are common knowledge as in the case partly considered by Mueller-Frank [8o]. 
arises from. Here, we show that heterogeneity in public beliefs hence actions (see section 5.5.2) can also arise from heterogeneity and uncertainty of prior beliefs.

Thirdly, this Chapter is related to the literature on agreement and disagreement under Bayesian rational learning (e.g Dixit and Weibull [34], Cripps et al. [31], Acemoglu et al. [1] and Sethi and Yildiz [103]). Cripps et al. [31] and Acemoglu et al. [1] study the validity of observational learning as a justification for the common prior assumption generally employed in most economics models. That is, individuals that share their experiences with each other will eventually have a shared history of events that are informative about the state of nature, and this will in turn lead to an agreement on their beliefs about the true state of nature. The framework employed by Cripps et al. [31] and Acemoglu et al. [1] to model this claim consists of two agents who observe a sequence of either private or public signals that are necessarily informative about the true state of nature. Cripps et al. [31] show that when both agents observe a sequence of correlated private signals then their beliefs converge to a common public belief provided that the signal space is finite. Acemoglu et al. [1] show that if agents start with heterogeneous prior beliefs, then observe a sequence of public signals and that they are uncertain about signal interpretation, then they do not necessarily converge to a common public belief. In our framework, rather than agents having to observe and learn from exogenously "communicated" signals, they instead learn endogenously through direct communication with their immediate neighbors in the network. Our framework for rational learning is thus similar to that of Cripps et al. [31] and Acemoglu et al. [1], whereby an infinite sequence of signals corresponds to an infinite set of agents each of whose private information is realized independently of the others. There is a difference in terms of the "time factor" though. In our framework, the number of signals an agent receives (by observing the neighbors announcements) at any given period $t$ depends on the number of $t$-order neighbors. As a consequence the speed of learning is generally faster (depending on the network topology) in our framework. Indeed, as in Cripps et al. [31] we find that provided that the distribution from which the signals are drawn has bounded variance, a consensus obtains in the limit of the number of agents. In relation to Acemoglu et al. [1], the uncertainty on how to interpret the signal is related to the case in which agents do not observe the prior beliefs of their neighbors. The uncertainty of neighbors' priors leads to uncertainty on how to interpret their announcements, which in turn leads to uncertainty on the signal interpretation. Contrary to Acemoglu et al. [1] we show that asymptotic consensus obtains provided the signal space is finite and that the distribution of prior beliefs, hence of expected sig- 
nals is bounded. Heterogeneity obtains otherwise. Additionally we show that under such conditions, a consensus also implies correct asymptotic beliefs.

Dixit and Weibull [34] and Sethi and Yildiz [103] study the polarization in public beliefs between two groups of agents when agents do not observe the prior beliefs of other agents outside of their group. They show how uncertainty of prior beliefs reinforces disagreement in opinions of the members of different groups. The findings in Dixit and Weibull [34] and Sethi and Yildiz [103] are special cases of this Chapter when the communication network is complete.

The closely related papers in the literature of bounded-rational learning are Demarzo et al. [32] and Golub and Jackson [49]. The main difference with what we do here is the assumption that agents start with private beliefs that are of finite precision. This assumption enables us to analyze the efficiency of information aggregation by public beliefs under bounded-rational learning and compare it with that under rational learning. In Proposition 11 we generalize the findings in Demarzo et al. [32], Golub and Jackson [49] concerning the convergent beliefs to dynamic networks. We show that the communication network does not necessarily have to be connected at all periods for a consensus to obtain. Specifically, a consensus in public beliefs will always emerge in a long-run provided that there exists a positive probability of realizing a connected network. Golub and Jackson [49] study conditions on the communication network for wisdom of crowds to obtain. Their definition of a wise crowd is closely related to our definition of correct asymptotic public beliefs in a weak sense (see Definition 12). They show that a society will be wise if for any finite-size subgroup of agents, the sum of weights connecting such a subgroup to the rest of the society is sufficiently large, and vice versa. That is, there should not be a subgroup of agents that stays prominent in the limit of population size. There are two major differences between our characterization of correct public beliefs from that of Golub and Jackson [49]. The first involves the initial conditions, whereby in Golub and Jackson [49] it is assumed that agents' prior beliefs are drawn from a normal distribution with mean equal to the true state of nature. On the contrary, we model a general case in which prior beliefs are distributed heterogeneously across the population. We then show that a necessary condition for public beliefs to correctly aggregate private information is for prior beliefs to be correlated with the true state of nature. In other words, the initial condition of Golub and Jackson [49] is derived as a necessary condition in our case. The second difference is that Golub and Jackson [49] characterize conditions on the network topology under which wisdom does not occur. On the contrary, we characterize conditions under which wisdom occurs and in Theorem 7 we show that a network must be asymptotically balanced 
for a society to be wise. To demonstrate the generality of our approach, we provide an example in which public beliefs are not correct under Golub and Jackson [49] but are correct in our case. We then further show as a corollary that correct public beliefs obtain in Erdös-Rényi family of random networks but not in networks formed through preferential attachment, like scale-free networks.

There is also a related literature on bounded-rational learning in physics and computer science which we shall not review in detail here. It focuses on conditions for a consensus to be attained contrary to this Chapter in which the primary goal is on the correctness of public beliefs and how it relates to rational learning. See Jackson [54] for more references on related models.

The rest of the chapter is organized as follows. In Section 5.2 we outline the framework of communication and learning, providing the informational and communication structure. Section 5.3 presents the characterization of the general structure of public beliefs for a finite population. Section 5.4 characterizes the conditions on the informational and communication network structures under which public beliefs correctly aggregate decentralize information. Section 5.5 deals with convergence rates and efficient information aggregation. All proofs are gathered in the Appendix.

\subsection{THE MODEL}

The set of agents is denoted by $N=\{1, \cdots, i, \cdots, n\}$. There is a state of nature $X$ that is unknown and unobservable to all agents. The true value of $X$ is $\bar{\mu}$ or generally a Dirac delta function centered at $\bar{\mu}$. Agents form and update beliefs about $X$. We assume without loss of generality that the prior belief of each $i \in N$ is a normal distribution with mean $\mu_{i}$ and unit variance. That is each $i \in N$ initially believes that $X$ is normally distributed with mean $\mu_{i, 0}$ and unit variance. The assumption of unit variance is for simplicity and does not affect the main conclusions of the paper.

We model agents' uncertainty of others' prior beliefs by assuming that they are normally distributed with mean $v_{i}$ for each $i \in N$ and variance-covariance matrix $M$ with entries $m_{i j} \geq 0$ for all $(i, j) \in N$. That is

$$
\mu_{0} \sim \mathscr{N}(\nu, M) \text { and } X \sim_{i} \mathscr{N}\left(\mu_{i, 0}, 1\right)
$$

where $\mu_{0}$ and $\boldsymbol{v}$ are column vectors of $\mu_{i, 0}$ and $v_{i}$ for all $i \in N$ respectively, and $\sim_{i}$ means "distribution according to $i$ ". For a clearer exposition, we refer to the pair $\left(\mu_{i, 0}, 1\right)$ as prior belief of $i \in N$ and the pair $\left(v_{i}, M_{i}\right)$, where $M_{i}$ is the $i$ th row of $M$, as $i$ 's prior belief distribution. When the prior beliefs are independently distributed then $i^{\prime}$ 's prior belief distribution will simply be the pair $\left(v_{i}, \eta^{2}\right)$, where $\eta^{2}$ is the associated 
variance. The assumption that $X$ is normally distributed is also for simplicity and does not affect our main results.

Given prior beliefs, each agent observes a private signal $s_{i}$ that is informative about $X$, and takes the form $s_{i}=X+\varepsilon_{i}$. Conditional on $\mu_{i, 0}$, each $i$ believes that $X$ and $\varepsilon_{1}, \cdots, \varepsilon_{n}$ are independently distributed, and it is common knowledge that $\varepsilon_{i} \sim \mathscr{N}\left(0, \sigma^{2}\right)$ for all $i \in N$ Agents therefore differ with respect to their prior beliefs and private information, and our goal is to study the public beliefs resulting from deliberation under general communication networks. In what follows, we denote by $\mu_{i, t}$ for the mean associated with the posterior belief of $i$ at period $t$, and by var $_{i, t}$ for the associated variance.

\subsubsection{Communication network}

The communication among agents is modeled through an associated network or graph. Let $G(n, E)$ be a graph with $n$ vertices representing the number of agents and $E$ is the set of edges linking different pairs of agents such that a graph $g_{i j}$ defines a communication link between $i$ and $j$. In particular if $g_{i j}>0$, then $j$ communicates to $i$, or simply that $i$ observes $j$ 's posterior beliefs, and $g_{i j}=0$ implies the absence of communication between $i$ and $j$. No strict restrictions are imposed on $g_{i j}$ 's except that $0 \leq g_{i j} \leq 1$. In particular it is not necessary that $g_{i j}=g_{j i}$. The corresponding adjacency matrix of interactions is denoted by G.7

The first-order neighborhood $N_{i, 1}$ is the set of agents that directly communicate with $i$. That is, $N_{i, 1}=\left\{j \in N ; g_{i j}>0\right\}$. The corresponding cardinality of $N_{i, 1}, k_{i, 1}=\# N_{i, 1}$, is the first-order degree of $i$. A path between $i$ and $j$ is a connected set of links $\mathscr{P}_{i j}=$ $\left\{g_{i 1}, g_{12}, \cdots, g_{(j-1) j}\right\}$ such that $g_{i j}>0$ for each $g_{i j} \in \mathscr{P}_{i j}$. The length of the path between $i$ and $j$ is denoted by $\left|\mathscr{P}_{i j}\right|$, which is simply the $L^{1}$ norm of $\mathscr{P}_{i j}$. We can then define the second-order neighborhood of $i, N_{i, 2}$ as the set of agents, indexed by $j_{2}$, such that for each $j_{2} \in N_{i, 2}$ there exists a path of length two between $i$ and $j$. That is $N_{i, 2}=\left\{j \in N ;\left|\mathscr{P}_{i j}\right|=2\right\}$. The second-order degree of $i$ is $k_{i, 2}=\# N_{i, 2}$. In a similar logic we can define the $t$-order neighborhood of $i$ as a set, $N_{i, t}=\left\{j \in N ;\left|\mathscr{P}_{i j}\right|=t\right\}$. The corresponding t-order degree is denoted by $k_{i, t}=\# N_{i, t}$.

Property 1. A communication network with the corresponding matrix $G$ is said to be connected if for any two agents $(i, j) \in N$, there exists a path of at least one step from $i$ to $j$ and vice versa.

7 We use $G$ to denote both the underlying network and the corresponding matrix unless otherwise specified. 
Definition 11: (a) A geodesic $d_{i j}(G)$ between two agents $(i, j) \in N$ is the shortest path between them. That is $d_{i j}(G)=\min \left\{\left|\mathscr{P}_{i j}\right|\right.$;for a pair of agents $\left.\{i, j\} \in N\right\}$.

(b) The diameter of a network $G, D(G)$ is its longest geodesic. That is

$$
D(G)=\max _{\{i, j\} \in N}\left\{\left|\mathscr{P}_{i j}\right| ; \forall i, j \in N\right\} \text {. }
$$

A network is said to be finite if its diameter is finite. Note that it is possible for the network to be finite when the population size is infinite.

\subsubsection{Dynamic networks}

We also consider dynamic communication structure in which the neighborhood of an agent changes over time. We do not define the specific mechanism by which agents switch their neighborhood but rather consider a generic and an arbitrary switching mechanism. Such mechanism can be strategic or simply random. The strategy can be a function of the current position in the network and/or other agents observable characteristics. See for example Sethi and Yildiz [104] for a switching mechanism that depends on the precision of opponents' private information, and König et al. [61] for a switching mechanism that depends on network related properties such as the centrality of an agent.

To be precise, let $\gamma$ be the switching strategy or simply the switching signal, defined as $\gamma: N \rightarrow G$. We then denote by $\mathscr{G}=\left\{G_{\gamma(1)}, \cdots, G_{\gamma(t)}, \cdots\right\}$ as a class/set of all possible networks or graphs that can be defined on the set of agents $N$ as a result of the switching strategy $\gamma \cdot G_{\gamma(t)}$ is therefore the resulting network at time $t$ from switching strategy $\gamma$. Under such dynamics, it is then possible that at certain periods some agents do not have any neighbors, that it $k_{i, 0}=0$.

A special case of this dynamic interaction structure includes that in which the switching strategy maps into the same network structure over time. That is $G_{\gamma(1)}=$ $\cdots=G_{\gamma(t)}=\cdots$. We denote such a switching strategy by $\gamma_{0}$. Another special case is when the switching strategy induces a connected network structure at each period. That is each $G_{\gamma(t)}$ for all $t$ is connected. Let such a switching strategy be denoted by $\gamma_{c}$

\subsubsection{Rational learning}

Under rational learning, given the prior beliefs and after observing the signal $s_{i}$, each $i \in N$ computes their posterior belief. The posterior beliefs are then truth- 
fully announced to the corresponding neighbors. After observing their neighbors' announcements, each $i \in N$ updates their belief and the resulting posterior beliefs are again simultaneously announced to the neighbors at the end of that period, and so forth. The sequential process continues until each agent's posterior announcements are constant, in which case each agent has no new information to learn from their neighbors. The limit belief of each $i \in N$ becomes common knowledge to his first-order neighbors, and we refer it as a local public belief or simply public belief. The crucial assumptions in the rational learning mechanism are common knowledge of rationality, and that agents have memory of the history of their neighbors announcements.

Example 6. Consider two agents $N=\{a, b\}$ such that $g_{a b}=g_{b a}=1$; an undirected link exists between $a$ and $b$. Let the prior beliefs be normally distributed such that $X \sim_{a}$ $\mathscr{N}\left(\mu_{a, 0}, 1\right)$ and $X \sim_{b} \mathscr{N}\left(\mu_{b, 0}, 1\right)$, and the private signals be $s_{a}=X+\varepsilon_{a}$ and $s_{b}=X+\varepsilon_{b}$ respectively. As defined above, assume that $X, \varepsilon_{a}, \varepsilon_{b}$ are independently distributed and that $\varepsilon_{a}$ and $\varepsilon_{b}$ are independent and identically distributed with $\varepsilon_{i} \sim \mathscr{N}\left(0, \sigma^{2}\right)$ for all $i=a, b$.

In the first round, after observing signals, it follows from Lemma 9 that the posterior beliefs of both agents become $X \sim_{i} \mathscr{N}\left(\mu_{i, 1}\right.$, var $\left._{i, 1}\right)$ for $i=a, b$. Where $\mu_{i, 1}=\frac{\sigma^{2}}{1+\sigma^{2}} \mu_{i, 0}+\frac{1}{1+\sigma^{2}} s_{i}$ and $v_{a r} r_{i, 1}=\frac{\sigma^{2}}{1+\sigma^{2}}$. At the end of the first round each agent announces their posterior beliefs. From $b$ 's announcement, a knows that

$$
\left(1+\sigma^{2}\right) \mu_{b, 1}=\sigma^{2} \mu_{b, 0}+s_{b}
$$

If we assume that a knows the prior belief of $b$, that is a knows that b's prior belief is normally distributed with mean $\mu_{b, 0}$ and unit variance, then a can deduce $s_{b}$ from the first period announcement of $b$. The similar argument follows for $b$ since $g_{b a}=1$. Combining their posterior beliefs at the end of the first period together with the deduced signals $s_{a}$ and $s_{b}$, both agents update their beliefs to $X \sim_{i} \mathscr{N}\left(\mu_{i, 2}\right.$, var $\left._{i, 2}\right)$ for $i=a, b$, where

$$
\mu_{i, 2}=\frac{\sigma^{2}}{2+\sigma^{2}} \mu_{i, 0}+\frac{1}{2+\sigma^{2}}\left(s_{a}+s_{b}\right), \quad \operatorname{var}_{i, 2}=\frac{\sigma^{2}}{2+\sigma^{2}}
$$

From the announcements at the end of the second period, both a and $b$ do not have anymore information to learn from each others' announcements. Learning stops, and their posterior beliefs become public information.

\subsubsection{Bounded-rational learning}

Under bounded-rational learning, agents update their beliefs sequentially just like in the case rational learning except that they are not able to disentangle between 
old and new information from announcements of their first-order neighbors. After receiving private signals, each agent updates their prior belief in accordance to Bayes rule as in Lemma 9. The resulting private beliefs are then communicated to the first-order neighbors. From the second period onwards, agents incorporate information from their first-order neighbors by simply taking the weighted average of their posterior beliefs. That is, at period $t i$ 's posterior mean is given by

$$
\mu_{i, t+1}=\sum_{j=1}^{N} g_{i j}(t) \mu_{j, t} \quad i=1, \cdots, n
$$

where $0 \leq g_{i j}(t) \leq 1$ is the weight that $i$ attaches to $j$ 's announcement at period $t$. Since each agents revises their belief in every period, it follows that $g_{i i}(t)>0$ for all $i \in N$ and $t \geq 0$. If $G_{\gamma(t)}$ is the associated matrix of interactions in the $t$-th period, then (5.2.2) can be written as

$$
\boldsymbol{\mu}_{t+1}=G_{\gamma(t)} \boldsymbol{\mu}_{t}
$$

where $\mu_{t}$ is a vector of posterior means in the $t$-th period. We assume that the same updating rule applies to even the variance of precision of agents beliefs.

\section{$5 \cdot 3$ PUBLIC BELIEFS}

In this section, we characterize the general structure of public beliefs under the informational structure described above. We focus of the case of a finite population and provide conditions for heterogeneity and consensus in public beliefs to obtain.

\subsubsection{Rational learning}

The following theorem establishes the structure of public beliefs under rational learning.

THEOREM 5: Let the communication network be common knowledge and connected. Let also $\mu_{i, \infty}$ and var ${ }_{i, \infty}$ denote the mean and variance of $i$ 's public belief respectively. Under rational learning the public belief of each $i \in N$ is normally distributed with mean and variance as follows.

(i) If for each $i \in N, \mu_{j, 0}$ for all $j \in N_{i, 1}$ are observable, then

$$
\mu_{i, \infty}=\frac{\sigma^{2}}{n+\sigma^{2}} \mu_{i, 0}+\frac{1}{n+\sigma^{2}} \sum_{j=0}^{n} s_{j} \quad \text { and } \quad \operatorname{var}_{i, \infty}=\frac{\sigma^{2}}{n+\sigma^{2}}
$$

for all $i \in N$, where $s_{0}=s_{i}$ 
(ii) If for each $i \in N, \mu_{j, 0}$ for all $j \in N_{i, 1}$ are unobservable but it is common knowledge that $\mu_{0} \sim \mathscr{N}\left(\boldsymbol{v}, \eta^{2} \mathbf{I}\right)$, where $\boldsymbol{\mu}_{0}$ is a column vector of all $\mu_{i, 0}$ and $\mathbf{I}$ is an $n \times n$ identity matrix, then

$\mu_{i, \infty}=\frac{\left(1+\sigma^{2}\right)\left(1+\eta^{2} \sigma^{2}\right)}{\left(1+\sigma^{2}\right)\left(1+\eta^{2} \sigma^{2}\right)+n-1} \mu_{i, 1}+\frac{1}{\left(1+\sigma^{2}\right)\left(1+\eta^{2} \sigma^{2}\right)+n-1} \sum_{l \in N \backslash\{i\}} \mathbb{E}_{i}\left[s_{l} \mid \boldsymbol{v}\right]$

and

$$
\operatorname{var}_{i, \infty}=\frac{\sigma^{2}\left(1+\eta^{2} \sigma^{2}\right)}{\left(1+\sigma^{2}\right)\left(1+\eta^{2} \sigma^{2}\right)+n-1}
$$

where $\mathbb{E}_{i}\left[s_{l} \mid v\right]=\sigma^{2}\left(\mu_{l, 0}-v_{l}\right)+s_{l}$ for all $i \in N$ and all $l \in N \backslash\{i\}$ is the expected signal of $l$ according to $i$ given $\boldsymbol{v}$.

(iii) If the communication network is complete and it is common knowledge that $\mu_{0} \sim$ $\mathscr{N}(v, M)$, where $m_{i j}>0$ for all $(i, j) \in N$, then $\mu_{i, \infty}$ and var $i_{i, \infty}$ are as in (5.3.1) above.

Proof. See Appendix 5.7.1

The first implication of Theorem $5(i)$ is that when agents observe their neighbors' prior beliefs and that the communication network is common knowledge and connected, public beliefs fully incorporate all private information. The second implication is that under similar conditions on the network and prior beliefs, if the realized prior beliefs are identical, then a consensus in public beliefs obtain. This result is consistent with that of the literature on common knowledge and consensus, for example Geanakoplos and Polemarchakis [47] and Parikh and Krasucki [92] in which they find that a consensus arises under a common prior assumption. Similarly in the literature of Bayesian rational learning on network for example Gale and Kariv [46] and Mueller-Frank [8o] it is established that a consensus (in actions) arises under common prior assumption and connectedness of the communication network. The only supplement to these papers in this regard is that Theorem $5(i)$ also emphasizes the fact that prior beliefs do not have to be common knowledge for consensus to obtain, but rather they are observable to the first-order neighbor.

The third implication of Theorem $5(i)$ is that heterogeneity in prior beliefs leads to heterogeneity in public beliefs. That is, let $\mu_{i, \infty}^{o}$ denote the public belief of $i$ when neighbor's prior are observable, then for any pair of agents $(i, j) \in N$ it follows from (5.3.1) that

$$
\mu_{i, \infty}^{o}-\mu_{j, \infty}^{o}=\frac{\sigma^{2}}{n+\sigma^{2}}\left(\mu_{i, 0}-\mu_{j, 0}\right)
$$

in which case only the heterogeneity in prior beliefs leads to heterogeneity in public beliefs. This result also complements the literature on rational learning which has focused mainly on learning under common prior beliefs. 
Theorem 5 (ii) establishes the structure of public beliefs when agents are uncertain about others' prior beliefs. Generally, public beliefs exhibit three sources of heterogeneity under uncertainty of prior beliefs. Heterogeneity could result from a difference in the realized priors and/or signals, as well as a difference in prior beliefs distributions if it exists. To see this notice that under assumptions of Theorem 5 (ii), for any pair of agents $(i, j) \in N, \mathbb{E}_{i}\left[s_{l} \mid v_{k}\right]=\mathbb{E}_{j}\left[s_{l} \mid v_{r}\right]=\sigma^{2}\left(\mu_{l, 0}-v_{l}\right)+s_{l}$ for each $k \in N_{i, 1}, r \in N_{j, 1}$ and all $l \in N$. Let $\mu_{i, \infty}^{u}$ denote the public belief of $i$ when neighbor's prior are unobservable, then for any pair of agents $(i, j) \in N$

$$
\mu_{i, \infty}^{u}-\mu_{j, \infty}^{u}=\frac{\sigma^{2}\left(\eta^{2} \sigma^{2}\left(\mu_{i, 0}-\mu_{j, 0}\right)+\eta^{2}\left(s_{i}-s_{j}\right)+\left(v_{i}-v_{j}\right)\right)}{\left(1+\sigma^{2}\right)\left(1+\eta^{2} \sigma^{2}\right)+n-1}
$$

Implying that if the distributions of prior beliefs are heterogeneous, then heterogeneity in public beliefs can still arise even under identical realized priors and signals.

In comparison to the case in which priors are observable, the precision of public beliefs when priors are unobservable is always lower. That is let $v_{i, \infty}^{o}$ and $v a r_{i, \infty}^{u}$ be the variance of $i$ 's public beliefs when prior beliefs are observable and unobservable respectively, then

$$
\operatorname{var}_{i, \infty}^{o}-\operatorname{var}_{i, \infty}^{u}=\frac{-\sigma^{2}(n-1)\left(\eta^{2} \sigma^{2}\right)}{\left(\sigma^{2}+n\right)\left(\left(1+\sigma^{2}\right)\left(1+\eta^{2} \sigma^{2}\right)+n-1\right)}<0
$$

This implies that agents' confidence in their beliefs at the end of the learning process is always lower when they are uncertain of their neighbors' prior beliefs compared to when they observe their neighbors' prior beliefs.

Theorem 5 (iii) entails two main implications. Since a pair of agents with an undirected link between them is a simplest form of a complete network, Theorem 5 (iii) implies that the findings in Geanakoplos and Polemarchakis [47] extend to the case in which agents are uncertain of other's prior beliefs provided that their prior beliefs are correlated. This is a particularly strong result given that the literature on knowledge and consensus has always emphasized the condition of common knowledge of prior beliefs as a prerequisite for a consensus to obtain. Note the distinction we make between common knowledge of prior beliefs and common knowledge of prior beliefs' distributions. Theorem 5 (iii) shows that the necessary condition for consensus to emerge under rational learning when the network is complete, is prior beliefs' to be correlated and their distributions to be common knowledge. The second implication of Theorem 5 (iii) is that provided the network is complete and that prior beliefs are correlated, then public beliefs fully incorporate dispersed private information.

We do not go into the detailed analysis of the outcomes of rational learning when the communication network is dynamic because the requirements on agents' knowledge of the network switching strategies amounts to assuming common knowledge 
of the network structure, and therefore does not affect the final outcome. To see this, notice that the assumption that agents recall the history of their neighbors' announcements is indispensable if they are to be able to deduce the private information (or the expected private information) of their $t$-order neighbors. This then requires agents to have knowledge of the past and current positions of all agents in the network, which is equivalent to assuming common knowledge of the network. On the other hand, when the communication network is common knowledge, its topology does not affect the general properties of public beliefs. Hence, under rational learning, whether or not the network is dynamic does not affect the general properties of public beliefs.

\subsubsection{Bounded-rational learning}

The dynamic system (5.2.3) can generally be treated as a non-homogeneous Markov chain, and the special case in which $\gamma=\gamma_{c}$ is a homogeneous Markov chain. The convergence properties of non-homogeneous Markov chains has been well established in the literature, and it particularly depends on the irreducibility and aperiodicity properties of the transition matrices $G_{\gamma(t)}$ for all $t \geq 0$. A Markov chain (transition matrix) is said to be irreducible if it is possible to make a transition from any one state to every other state, not necessarily in one time step. In the context of communication networks described in section 5.2.1, irreducibility of the network or the matrix induced by the network implies that a path $\mathscr{P}_{i j}$ between any pair of agents $(i, j) \in N$ exists. Aperiodicity on the hand implies that there does not exists two or more groups of agents for which communication is possible only among groups and not within groups. For the communication structure considered in this Chapter, aperiodicity is guaranteed since self loops exists. A more general property of Markov chains that guarantees convergence to well defined characteristics is that of ergodicity. Ergodic Markov chains are irreducible, aperiodic and recurrent. A Markov chain is recurrent if every state is revisited infinitely many times. The following lemma summarizes the properties of ergodic Markov chains and at the same time acts as a definition for an ergodic chain.

LemMA 12: Let $\mathscr{G}=\left\{G_{\gamma(t)}\right\}_{t \geq 0}$ be (an infinite) sequence of finite matrices. If a Markov chain formed as a product of matrices in $\mathscr{G}$ is ergodic, then

$$
\lim _{T \rightarrow \infty} \prod_{t=0}^{T} G_{\gamma(t)}=\mathbf{e v}^{\prime}
$$

where $\mathbf{e}$ is a column vector of ones, $\mathbf{v}$ is a column vector of real values and $\mathbf{v}^{\prime}$ is the transpose of $\mathbf{v}$. 
Proof. The proof can be found in Wolfowitz [112].

Note that $\mathbf{e v}^{\prime}$ is an $n \times n$ matrix with identical rows, hence it is of unit rank. The analysis of the convergence of (5.2.3) then reduces to determining the conditions under which a sequence of communication networks induced by a switching strategy $\gamma$ lead to an ergodic Markov chain. The following proposition provides such conditions.

Proposition 11: Let $\tau \geq 0$ be a sufficiently large integer. If the switching strategy $\gamma$ is such that in every time interval $[t, t+\tau)$ a connected communication network obtains, then

$$
\mu_{i, \infty}=\left[\lim _{T \rightarrow \infty} G^{T} \boldsymbol{\mu}_{1}\right]_{i}=\mathbf{v}^{\prime} \boldsymbol{\mu}_{1} \quad \text { for every } i \in N
$$

where $G^{T}=\prod_{t=0}^{T} G_{\gamma(t)}$.

Proof. See Appendix 5.7.2.

Proposition 11 states that under some restrictions on the switching strategy, specifically that there be a positive probability of realizing a connected network, a consensus in public beliefs obtains. The public beliefs are a weighted average of the initial private beliefs. The weights vector $\mathbf{v}$ generally depends on the network structure. To understand the nature of the weights vector let us consider the special case in which $\gamma=\gamma_{c}$, that is in which $\gamma$ maps into the same network topology. This special case is that of static networks studied in Demarzo et al. [32] and Golub and Jackson [49], in which case the weight vector $\mathbf{v}$ is related to the left eigenvector of $G_{\gamma_{c}}$ associated with the leading eigenvalue. The left eigenvector is normally associated with the measure of centrality; the eigenvector centrality (see Bonacich and Lloyd [22] and the references therein). The centrality measures quantifies the level of influence of each agent. That is for each $i \in N$, the $i$ th value of $\mathbf{v}, v_{i}$ is the measure of how influential $i$ is in shaping public beliefs. The composition of the vector $\mathbf{v}$ is thus central in determining the correctness and quality of public beliefs. For a detailed exposition of the nature of the weight vector $\mathbf{v}$ we refer the reader to Demarzo et al. [32] and Golub and Jackson [49], we only provide a simple example below.

Example 7. Let $G_{1}$ denote the associated transition matrix of the communication network in Figure $12 a$ and $G_{2}$ for the that in Figure $12 b$, and let the corresponding weights vectors be $\mathbf{v}_{1}$ and $\mathbf{v}_{2}$ respectively. A power iteration of each transition matrix results into

$$
\mathbf{v}_{1}=(0.363,0.204,0.191,0.073,0.121,0.048) \quad \text { and } \quad \mathbf{v}_{2}=(0,0,0,0.359,0.381,0.260) \text {. }
$$

Clearly, the influence of each agent depends on their first-order connectivity and the their closeness to other agents who are also have high first order-connectivity. Take for example 


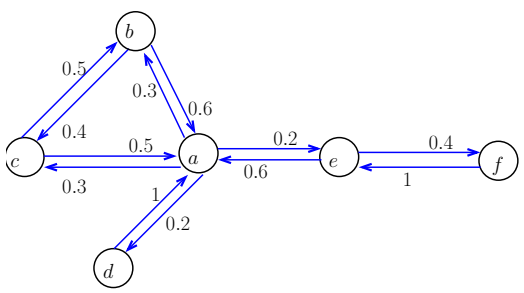

(a) A connected network: $G_{1}$

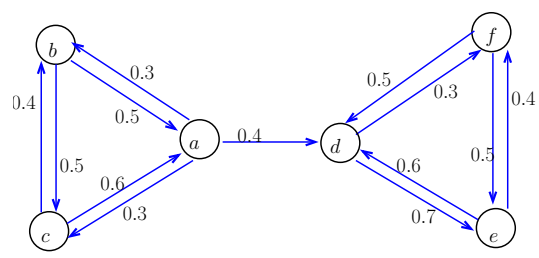

(b) Network with prominent "family": $G_{2}$

Figure 12: The labels on each link is the weight a player attaches to the neighbors opinions.

the network $G_{1}$ of Figure $12 a$ in which agents $d$ and $f$ both observe announcements of only one other agent and both communicate to one other agent. Though the first-order neighbor of agent $f$ attaches more weight to $f^{\prime}$ s announcements than does the first-order neighbor of agent $d$, agent $d$ is more influential than $f$ in a long-run. This is precisely the effect of being connected to other agents who are themselves have higher first-order connectivity, as is the case of $a$ and $e$ who are the first-order neighbors of $d$ and $f$ respectively.

In the case of communication network in Figure 12b, there are two subgroups (that is $\{a, b, c\}$ and $\{d, e, f\})$ each of whom form a complete subgroup. The inter-subgroup communication on the other hand is unidirectional, that is members of subgroup $\{a, b, c\}$ observe and learn from announcements of those in subgroup $\{d, e, f\}$ and not vise versa. As a consequence, a consensus emerges in a long-run in which member of subgroup $\{a, b, c\}$ adopt public beliefs of subgroup $\{d, e, f\}$. This example highlights the effect of the presence of prominent "families" discussed in Bala and Goyal [12] in a different model but related in the sense that they also assume bounded-rationality of among as in this case.

In general, under bounded-rational learning public beliefs are greatly influenced by the topology of the communication network and the distribution of private information. That is public belief of $i$ will be normally distributed with mean

$$
\mu_{i, \infty}=\sum_{j=1}^{n} v_{j}\left(\frac{\sigma^{2}}{1+\sigma^{2}} \mu_{i, 0}+\frac{1}{1+\sigma^{2}} s_{j}\right) .
$$

and variance

$$
\operatorname{var}_{i, 1}=\sum_{j=1}^{n} v_{j} \frac{\sigma^{2}}{1+\sigma^{2}}=\frac{\sigma^{2}}{1+\sigma^{2}}
$$

The implication is that, under bounded-rational learning, if agents start with an identical level of precision or confidence in their beliefs, they stay so even after the learning process has ended. This result highlights the weakness associated with bounded- 
rational learning in correct aggregation of private information as will be elaborated in the following subsection.

\subsection{CORRECT PUBLIC BELIEFS}

Correctness of public beliefs generally depends on whether or not they fully incorporate private information of all other agents and not just of their first-order neighbors. If private information of all agents is fully incorporated then we would expect the public beliefs to converge jointly in probability for large $n$ to the true state of nature. We differentiate correctness in public beliefs for finite population from that when the population size is infinite. For a clear distinction, we refer to beliefs resulting from learning as the population size goes to infinity as asymptotic public beliefs as opposed to just public beliefs for finite population. Public beliefs are said to be correct if they fully incorporate private information of all other agents. In the case of asymptotic public beliefs we differentiate between correctness in a weak and strong sense. That is, public beliefs are correct in a strong sense if for each agent it converges in probability to a Dirac delta function centered at the true state of nature $\bar{\mu}$, and in the weak sense if it converges in probability to a normal distribution centered at the true state of nature and not necessarily zero variance. Based on these definitions it is easy to see that the basic requirement for both public beliefs and asymptotic public beliefs to be correct is the communication network to be connected. We therefore will not repeatedly emphasize this fact in most of the analysis that follows.

In the case of rational learning we have already provided conditions for public beliefs to fully incorporate private information of other agents, so we will now focus on correctness of asymptotic public beliefs below. To precisely define convergence in probability, we need to define a sequence of networks for a growing population size $n$. We let $G(n)$ denote the network or equivalently the corresponding matrix when the population size is $n$. This then implies that there are two limits in the learning processes above; the limit for time $t$ and for population size $n$. To study such dynamic processes, we have to assume that one of the two limits is reached faster than the other. Alternatively, and perhaps even more intuitive, we first fix the network topology and derive the corresponding nature of public beliefs, then study the evolution of public beliefs for a growing population size. The population size should grow in such a way that the topology (or at least the main characteristics) of the communication network are preserved. Given the nature of public beliefs, we then study the sequence of networks $\{G(n)\}_{n \geq 2}$ of fixed topology and show conditions under which convergence in probability of public beliefs to the true state 
of nature occurs. We formally define strong and weak correctness of asymptotic public beliefs as follows.

DeFINITION 12: (a) Asymptotic public beliefs are said to be correct in a strong sense if for each $i \in N$

$$
\lim _{n \rightarrow \infty} \mathbb{P}\left(\left|\mu_{i, \infty}(n)-\bar{\mu}\right|>\epsilon\right)=0 \text { and } \lim _{n \rightarrow \infty} \operatorname{var}_{i, \infty}(n)=0
$$

for all possible realizations of $\mu_{i, 0}$.

(b) Asymptotic public beliefs are said to be correct in a weak sense if for each $i \in N$, condition (5.4.1) is satisfied but

$$
\lim _{n \rightarrow \infty} \operatorname{var}_{i, \infty}(n)<\infty
$$

Under strong correctness of asymptotic public beliefs we require that each agent's public belief converges in probability precisely to the true state of nature for all possible realizations of $\mu_{i, 0}$. For weak correctness, the asymptotic public belief of each agent must place the most weight on the true state of nature for all possible realizations of $\mu_{i, 0}$. With these definitions, if public beliefs are correct in a strong sense then they are also correct in a weak sense.

\subsubsection{Rational learning}

As shown in Example 6 and Theorem 5, full incorporation of private information by public beliefs in the case of rational learning depends on the observability of prior beliefs. We show below that in addition to observability of prior beliefs, correctness of asymptotic public beliefs also depends on the structure of prior beliefs and of private information, and in some cases on the structure of the communication network. The following theorem establishes necessary conditions for asymptotic public beliefs to be correct.

THeORem 6: As a general condition, let the sequence $\{G(n)\}_{n \geq 2}$ be common knowledge.

(i) When it is common knowledge that $\mu_{0} \sim \mathscr{N}\left(\nu, \eta^{2} \mathbf{I}\right)$, then $\mu_{i, \infty}(n) \stackrel{p}{\rightarrow} \bar{\mu}$ and

$$
\lim _{n \rightarrow \infty} \operatorname{var}_{i, \infty}(n)=0 \quad \forall i \in N \text { if } \sigma^{2} \text { and } \eta^{2} \text { are finite. }
$$

(ii) When it is common knowledge that $\mu_{0} \sim \mathscr{N}(\boldsymbol{v}, M)$, where $m_{i j}>0$ for all $(i, j) \in N$, then $\mu_{i, \infty}(n) \stackrel{p}{\rightarrow} \bar{\mu}$ and $\lim _{n \rightarrow \infty} \operatorname{var}_{i, \infty}(n)=0 \forall i \in N$ if the communication network is complete and that $m_{i i}<\infty$. 
Proof. See Appendix 5.7.3.

The general implication of Theorem 6 is that whenever asymptotic public beliefs are correct, they are correct in a strong sense. Depending on the observability of prior beliefs though, there exists a difference on the speed of convergence to correct asymptotic public beliefs, which is determined by the rate at which the variance of an agent's belief goes to zero. As indicated in the discussion of Theorem 5 , the variance associated with an agent's public belief for a given population size depends on the observability of prior beliefs.

Theorem $6(i)$ shows that despite the difference in the speed of convergence, correct asymptotic public beliefs obtain irrespective of uncertainty of others' prior beliefs provided it is common knowledge that prior beliefs are independently distributed, and that the signal space and prior beliefs space are finite. That is $\sigma^{2}<\infty$ and $\eta^{2}<\infty$. Note that in Theorem 6, correct asymptotic public beliefs also implies consensus in asymptotic public beliefs. The condition that the signal space must be finite for correct learning (public beliefs in this case) to occur has been pointed out in other models of learning in the literature. For example Cripps et al. [31], in which they show that a finite signal space is necessary for a consensus to obtain in a learning model where two agents observe and learn from a sequence of correlated private signals. Similarly in the literature of sequential Bayesian learning, it is shown that boundedness of private beliefs plays a role in determining whether or not wrong herds will emerge [109]. Recall that a private belief is what results after agents incorporate their realized signal into their prior belief, so in essence if the signal space is finite then private beliefs are also finite/bounded.

Our claim that correct asymptotic public beliefs obtain irrespective of uncertainty of prior beliefs provided that prior beliefs are independently distributed and that the signal and prior beliefs spaces are finite, is a particularly strong result. Since uncertainty in prior beliefs in turn leads to uncertainty in the signal interpretation, one would expect that the uncertainty in signal interpretation should derail correctness in asymptotic public beliefs. Although our framework is different, this finding is contrary to Acemoglu et al. [1] who find that when two agents learn from a sequence of signals and that they are uncertain of signal interpretation, then their beliefs do not necessarily converge to a consensus (which is equivalent to correct public beliefs in our case). We find that asymptotic consensus fails to arise only if the distribution of prior beliefs, hence of expected signals is not bounded.

Theorem 6 (ii) establishes the basic condition for asymptotic public beliefs to be correct when the distributions of prior beliefs are correlated and that the correlation 
coefficients are not necessarily finite. In particular, the variance of the prior belief of each agent must be finite and that the communication network is complete.

\subsubsection{Bounded rational learning}

Under bounded-rational learning, the network topology almost solely determines the nature of public beliefs and should thus equally influence their correctness. Since a consensus exists in public beliefs, we can focus on the correctness of the public belief of a single agent for all possible realizations of prior beliefs. Under boundedrational learning, since agents incorporate others' beliefs by taking weighted averages, it follows that the public belief of an agent is said to be correct if it attaches equal weight to the private beliefs of all other agents. The following definition formalizes this observation.

DeFINITION 13: Under bounded-rational learning, public beliefs are said to be correct or fully aggregate private information if $\mu_{i, \infty}(n)=\operatorname{Ave}\left[\boldsymbol{\mu}_{1}\right]$ and that var $_{i, \infty}(n)<\infty$ for each $i \in N$, where

$$
\operatorname{Ave}\left[\boldsymbol{\mu}_{1}\right]=\frac{1}{n} \sum_{i=1}^{n} \mu_{i, 1} .
$$

The following additional definitions are useful for a complete characterization of the results below.

Definition 14: A matrix $G(n)$ is said to be doubly stochastic if $\sum_{j=1}^{n} g_{i j}(n)=\sum_{i=1}^{n} g_{i j}(n)=$ 1 for all $(i, j) \in N$

Definition 15: (a) A communication network is said to be perfectly balanced if the corresponding matrix $G(n)$ is doubly stochastic.

(b) A sequence of networks $\{G(n)\}_{n \geq 2}$ is said to be asymptotically balanced if $\lim _{n \rightarrow \infty} G(n)=$ $S$, where $S$ is an arbitrary doubly stochastic matrix.

THEOREM 7: Under bounded-rational learning, if $\left\{\mu_{i, \infty}(n)\right\}_{n \geq 2}$ for each $i \in N$ is the sequence of public beliefs corresponding to the sequence $\{G(n)\}_{n \geq 2}$ of networks, then

(i) $\mu_{i, \infty}(n)=\operatorname{Ave}\left[\mu_{1}\right]$ and $\operatorname{var}_{i, \infty}(n)<\infty \forall i \in N$ if and only if $\sum_{j=1}^{n} g_{i j}(n)=$ $\sum_{i=1}^{n} g_{i j}(n)$ for all $(i, j) \in N$.

(ii) $\mu_{i, \infty}(n) \stackrel{p}{\rightarrow} \bar{\mu}$ and $\lim _{n \rightarrow \infty} \operatorname{var}_{i, \infty}(n)=\frac{\sigma}{1+\sigma} \forall i \in N$ if and only if $\lim _{n \rightarrow \infty} G(n)=S$, $\mu_{0} \sim \mathscr{N}\left(\bar{\mu}, \eta^{2} \mathbf{I}\right)$, and that $\sigma^{2}$ and $\eta^{2}$ are finite. Where $\bar{\mu}$ is an $n$ dimensional vector of $\bar{\mu}$. 
Theorem 7 (i) implies that under bounded-rational learning, correct public beliefs obtain if and only if the network is balanced. The extensiveness of the class of networks that satisfy the balancedness condition depends on whether or not selfloops are permitted to be heterogeneous. If the communication structure is such that all agents must place the same weight on their beliefs (that is $g_{11}=\cdots=g_{n n}$ ), then there are limited network topologies that satisfy balancedness. If on the other hand the weights $g_{i i}$ for all agents are permitted to be heterogeneous then there exists a wide range of networks that satisfy the balancedness condition. This can be checked by comparing the first-order influences using the balancedness condition, that is $\sum_{i=1}^{n} g_{i j}=\sum_{i=1}^{n} g_{i k}$ for $j \neq k$. Which is equivalent to

$$
g_{j j}+\sum_{i \neq j} g_{i j}=g_{k k}+\sum_{i \neq k} g_{i k}
$$

If $g_{j j}=g_{k k}$ for all $(j, k) \in N$ then it must be that $\sum_{i \neq j} g_{i j}=\sum_{i \neq k} g_{i k}$, which implies that all $(j, k) \in N$ must assign weights in an identical manner though not necessarily to the same first-order neighbors. If on the contrary $g_{j j} \neq g_{k k}$ for all $(j, k) \in N$ such that $\sum_{i \neq j} g_{i j} \neq \sum_{i \neq k} g_{i k}$ for all $(j, k) \in N$, then agents can assign weights in different and various ways.

Theorem 7 (ii) has three main implications. First, under bounded-rational learning asymptotic public beliefs can only be correct in a weak sense. Once agents start with beliefs that are not completely precise (in the sense that the variance of prior beliefs is greater than zero), they will not be able to learn the true state of nature with complete precision as in the case of rational learning. Secondly, unlike in the case of rational learning where the distribution of prior beliefs can be independent of the true state of nature, under bounded-rational learning asymptotic public beliefs can only be correct if both the signals and prior beliefs are informative about the true state of nature. That is the prior belief of each agent must be normally distributed with mean equal to the true state of nature and finite variance. This restrictive condition highlights the superiority of rational learning in aggregating privately information compared to bounded rational learning. Third, correct asymptotic public beliefs obtain if and only if the network is asymptotically balanced. The range of networks that are asymptotically balanced is wide and we explore their characterization in the following subsection.

\subsubsection{Asymptotically balanced networks}

To characterize the class of communication networks that are asymptotically balanced, we need to construct a measure of balancedness of a network/matrix. We 
denote by $\phi(n)$ for a network $G(n)$ as a measure of its balancedness defined as follows.

Definition 16: Let $S(n)$ be closest doubly stochastic matrix in terms of the Frobenius norm to the matrix $G(n)$. Then $G(n)$ is said to be $\phi(n)$-balanced if given $S(n)$,

$$
\|S(n)-G(n)\|_{F}=\phi(n)
$$

where $\|.\|_{F}$ is the Frobenius norm.

We can then rephrase definition 15 (b) as saying that a sequence $\{G(n)\}_{n \geq 2}$ is asymptotically balanced if

$$
\lim _{n \rightarrow \infty} \phi(n)=\lim _{n \rightarrow \infty}\|S(n)-G(n)\|_{F}=0
$$

The following proposition provides an expression for $\phi(n)$ for any stochastic matrix of size $n$.

Proposition 12: Let $G(n)$ with entries $g_{i j}(n)$ for $(i, j) \in N$ be a stochastic matrix corresponding to a communication network whose measure of balancedness $\phi(n)>0$. Then

$$
\phi^{2}(n)=\frac{1}{n} \sum_{j=1}^{n}\left(1-\sum_{i=1}^{n} g_{i j}(n)\right)^{2}
$$

Proof. See Appendix 5.7.5.

Equation (5.4.3) gives an expression for the measure of balancedness of any arbitrary stochastic matrix. The quantity $\sum_{i=1}^{N} g_{i j}(n)=d_{j}^{i n}(n)$ is the in-degree of agent $j$. It specifies the influence of $j$ on her first-order neighbors' beliefs. Unlike the out-degree $d_{i}^{\text {out }}(n)=\sum_{j=1}^{N} g_{i j}(n)$ that is normalized to unity for all agents, the in-degree can generally be equal to, less or greater than unity. The higher the value of $d_{j}^{i n}(n)$, the more influential $j$ in terms of first-order influence. For various values of $n, d_{j}^{i n}(n)$ for each $j$ is a random variable whose distribution depends on how the communication network scales with $n$. For a sequence of networks $\{G(n)\}_{n \geq 2}$ there exists a corresponding sequence $\left\{d_{i}^{i n}(n)\right\}_{n \geq 2}$ of in-degree for each $i \in N$. If we define $d_{\text {max }}^{\text {in }}(n)=\max _{i \in N}\left\{d_{i}^{i n}(n) ; i \in N\right\}_{n \geq 2}$, then for each sequence $\{G(n)\}_{n \geq 2}$ there exists a corresponding sequence $\left\{d_{\text {max }}^{\text {max }}(n)\right\}_{n \geq 2}$.

The following theorem employs Proposition 12 to characterize conditions for a sequence of communication networks to be asymptotically balanced.

THEOREM 8: A sequence of networks $\{G(n)\}_{n \geq 2}$ with the corresponding sequence of maximum in-degrees $\left\{d_{\max }^{\text {in }}(n)\right\}_{n \geq 2}$ is asymptotically balanced if and only if 
(i) $\lim _{n \rightarrow \infty} d_{\text {max }}^{\text {in }}(n)=1$

(ii) there exists an integer $n^{\prime}>0$ such that for all $n>n^{\prime}$ the quantity

$$
h(n)=\sum_{j=1}^{n}\left(1-\sum_{i=1}^{n} g_{i j}(n)\right)^{2}
$$

stays constant.

Proof. See Appendix 5.7.6.

There exists a wide range of network topologies that satisfy condition $(i)$ in Theorem 8 , including random networks (graphs). To see this, notice that Theorem 8 (i) also implies that the sequence of the influence of the most influential agent $\left\{v_{\text {max }}(n)\right\}_{n \geq 2}$, where $v_{\max }(n)=\max _{i \in N}\left\{v_{i}(n) ; i \in N\right\}$ must converge to zero for large $n$ if the sequence $\{G(n)\}_{n \geq 2}$ is to be asymptotically balanced. This argument follows directly from the fact that the most influential agents are either those that are the most connected or those that are connected to the most connected agents (see example 7). Now consider the Erdös-Rényi family of random networks with parameters $n$ and $p$ (that is a random network $G(n, p)$ of $n$ agents in which each link is included in the network with probability $p$ independently of the other links), it is shown that for $p \geq \frac{\log ^{6} n}{n}$ and for all $i \in N$ [75]

$$
c \frac{1}{\sqrt{n}} \frac{\log n}{\log (n p)} \sqrt{\frac{\log n}{n p}}-\frac{1}{\sqrt{n}} \leq v_{1}(i) \leq c \frac{1}{\sqrt{n}} \frac{\log n}{\log (n p)} \sqrt{\frac{\log n}{n p}}+\frac{1}{\sqrt{n}}
$$

with probability $1-o(1)$, where $c>0$ is some constant. Clearly, for a sequence of such graphs it follows that $\lim _{n \rightarrow \infty} v_{\max }(n)=0$.

The class of communication networks that is ruled out by Theorem $8(i)$ is those that are formed through preferential attachment, such as scale-free and generally high-clustering networks. In such networks, $d_{\text {max }}^{\text {in }}(n):=f(n)$, where $f$ is an increasing function in its argument. That is $d_{\text {max }}^{\text {in }}(n)$ increases with $n$.

The following examples illustrate more other general network topologies that satisfy the two conditions for asymptotic balancedness in Theorem 8 and one which does not.

Example 8. (a) Consider a communication network that assumes a one dimensional lattice structure in Figure 13. This example is adapted from Golub and Jackson [49] to demonstrate the generality of our Theorem 8 compared to the characterizations of wisdom of crowds in Golub and Jackson [49]. They show that a society assuming a topology in Figure 13 is not wise (that is public beliefs do not correctly aggregate private information), but we demonstrate the contrary using Theorem 8. 


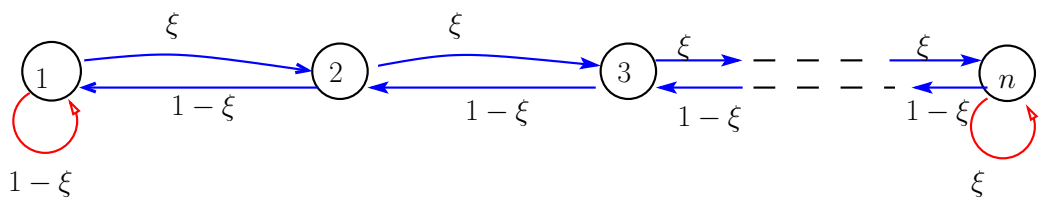

Figure 13: A network satisfying condition (ii) of Theorem 8

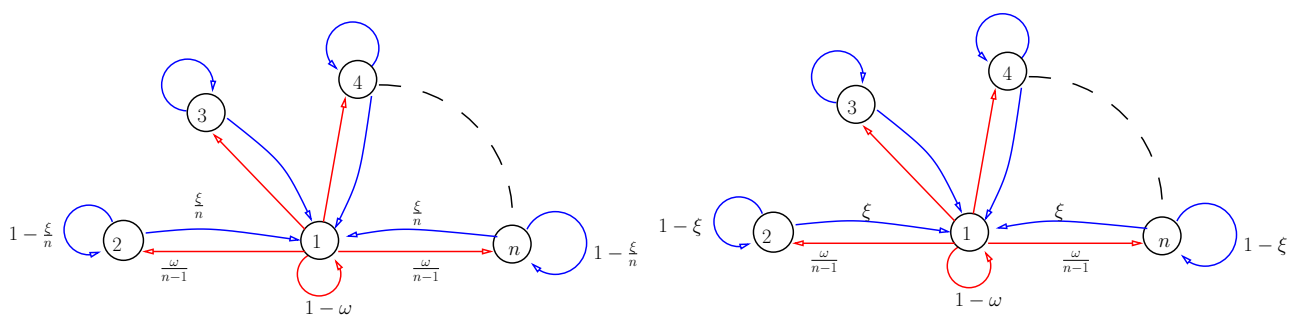

(a) Each agents influence decays with $n$

(b) Player I's influence grows with $n$

Figure 14: Asymptotically balanced networks.

It is easy to see that in such a network, the quantity $h(n)$ is constant and that

$$
\phi^{2}(n)=\frac{2(1-2 \xi)^{2}}{n}
$$

Implying that $\lim _{n \rightarrow \infty} \phi(n)=0$, hence public beliefs correctly aggregate private information.

(b) Consider the following two cases in which $h(n)$ is not independent of $n$. In the communication network topology of Figure 14a, each agent's first-order influence decays with $n$. The corresponding $\phi^{2}(n)$ is

$$
\phi^{2}(n)=\frac{1}{n}\left[\left(\omega-\frac{n-1}{n} \xi\right)^{2}+(n-1)\left(\frac{\xi}{n}-\frac{\omega}{n-1}\right)^{2}\right]
$$

Consequently, $\lim _{n \rightarrow \infty} \phi(n)=0$, in which case asymptotic balancedness obtains.

For the communication network of Figure $14 b$

$$
\phi^{2}(n)=\frac{1}{n}\left[(\omega-(n-1) \xi)^{2}+(n-1)\left(\xi-\frac{\omega}{n-1}\right)^{2}\right]
$$


in which case $\lim _{n \rightarrow \infty} \phi(n)=\infty$. This is a general situation in which an agent or a subgroup of agents posses unbounded influence. In such communication networks asymptotic balancedness fails.

\subsection{CONVERGENCE RATE AND EFFICIENT INFORMATION AGGREGATION}

\subsubsection{Convergence rate}

In both learning mechanisms, the convergence rate depends solely on the topology of the communication network. The following proposition summarizes the convergence rates in terms of network properties.

Proposition 13: Let $G_{\gamma(t)}=G_{\gamma_{c}}$ for all $t \geq 0$, where $G_{\gamma_{c}}$ is the transition matrix resulting from a communication network. Let $\lambda_{2}\left(G_{\gamma_{c}}\right)$ and $D\left(G_{\gamma_{c}}\right)$ be the second largest eigenvalue and the diameter of $G_{\gamma_{c}}$ respectively. Denote by $r_{R}$ and $r_{B R}$ for the convergence rates of rational and bounded-rational learning respectively, then

$$
r_{R}=\frac{1}{D\left(G_{\gamma_{c}}\right)} \geq \frac{\ln \left(1 / \lambda_{2}\left(G_{\gamma_{c}}\right)\right)}{\ln (c)}>\lambda_{2}\left(G_{\gamma_{c}}\right)=r_{B R}
$$

Proof. See Appendix 5.7.7.

Proposition 13 shows that rational learning generally converges faster than boundedrational learning. Specifically, under rational learning, it is the diameter of the communication network that matters and all other properties such as clustering and skewness of degree distributions do not matter as much. In the case of boundedrational learning, clustering and the nature of degree distributions matter since the second eigenvalue depends on them. For example, a network with a given number of links in which agents are clustered into subgroups with few (or weak) connections between subgroups generally has a higher second eigenvalue than a network with equal number of agents and edges but in which edges are randomly distributed. Similarly, a network formed by removing links from a "parent" network generally has a higher second eigenvalue than its "parent" network. This argument follows from the well known concept of interlacing eigenvalues according to Fiedler [40]. Finally if agents are highly "inward-looking" when updating their beliefs, that is for each agent the weight of a self-loop is higher compared to the total weight of outgoing links, then such a network also has a higher second eigenvalue, hence slow convergence rate. 
The relevance of the network topologies in determining the convergence rate becomes more apparent in decision environments with discounted payoffs as will be demonstrated in the subsection below.

\subsubsection{Efficient information aggregation}

To establish the efficiency of the two learning mechanisms and that of the network topologies in aggregating information, we introduce a simple decision problem in which agents' objective is to minimize the expected loss from mismatching their action and the true state of nature. Specifically, each agent has two choices in each period; either to take an action $a \in A_{i}$, where $A_{i}$ is a continuous action space for $i$, or "wait" for the next period(s). The joint action space (which we assume to be homogeneous for all agents, $A_{1}=\cdots=A_{n}$ ) for each agent is thus $\mathscr{A}=\{A, w\}$, where $w$ stands for "wait". If an agent takes an action within the action space $A$, he "exits" the game, meaning that he no longer learns and transmits new information but only transmits the same information he possesses at the time of exit. If on the other hand the agent decides to wait, then his expected loss for the next period is increased by a factor of $\frac{1}{\delta}$, where $0 \leq \delta \leq 1$. That is

$$
U_{i t}\left(\tilde{a}_{i t}, X\right)= \begin{cases}-\frac{1}{\delta^{t}} \mathbb{E}_{i, t}\left[\left(\tilde{a}_{i t}-X\right)^{2}\right] & \text { if } \tilde{a}_{i t}=a \in A \\ 0 & \text { if } \tilde{a}_{i t}=w\end{cases}
$$

where $\mathbb{E}_{i, t}$ stands for expectation according to agent $i$ at period $t$ and $\tilde{a} \in \mathscr{A}$. We assume for simplicity that agents are homogeneous in terms of payoff structure. The strategy of each agent thus entails choosing the optimal period to exit the game.

Under this setup, it is easy to see that the optimal action $a \in A$ for each agent at any given period is

$$
a_{i t}^{*}=\mathbb{E}_{i, t}[X]=\mu_{i, t}
$$

and the payoff corresponding to the optimal action is

$$
U_{i t}\left(a_{i t}^{*}, X\right)=-\frac{1}{\delta^{t}} \mathbb{E}_{i, t}\left[\left(\mu_{i, t}-X\right)^{2}\right]=-\frac{1}{\delta^{t}} \operatorname{var}_{i, t}
$$

where $v a r_{i, t}$ is the variance of $X$ according to $i$ at period $t$. This implies that an agent's exit time depends on his confidence in his beliefs.

We can also define the associated expected social welfare as the average of individual optimal expected utilities as follows.

$$
W_{t}(X)=\frac{1}{n} \sum_{i=1}^{n} U_{i t}\left(a_{i t}^{*}, X\right)=-\frac{1}{n \delta^{t}} \sum_{i=1}^{n} \mathbb{E}_{i, t}\left[\left(\mu_{i, t}-X\right)^{2}\right]
$$


Consider first the case in which there is no cost on the payoffs associated with waiting, that is when $\delta=1$. It is clear that it is optimal for agents to wait until all information has been exchanged among all agents, which in the case of boundedrational learning implies until a consensus is reached. Now, consider a balanced network (which supports correct information aggregation under bounded-rational learning) of size $n$. The optimal action (taken at the end of information exchange process) under rational learning when priors are observable is

$$
a_{i \infty}^{*}=\frac{\sigma^{2}}{n+\sigma^{2}} \mu_{i, 0}+\frac{1}{n+\sigma^{2}} \sum_{j=0}^{n} s_{j}
$$

and the corresponding expected social welfare $W_{\infty}(X)$ is

$$
W_{\infty}(X)=-\frac{1}{n} \sum_{i=1}^{n} \mathbb{E}_{i, t}\left[\left(a_{i \infty}^{*}-X\right)^{2}\right]=-\frac{1}{n} \sum_{i=1}^{n} \operatorname{var}_{i, \infty}=-\frac{\sigma^{2}}{n+\sigma^{2}}
$$

where the last equality follows from Theorem $5(i)$. Similarly for bounded-rational learning, the optimal action is

$$
a_{i \infty}^{*}=\frac{1}{n} \sum_{j=1}^{n}\left(\frac{\sigma^{2}}{1+\sigma^{2}} \mu_{i, 0}+\frac{1}{1+\sigma^{2}} s_{j}\right) .
$$

where we have used the fact that for a balanced network of size $n$, the weights or influence vector is $\mathbf{v}=\left(\frac{1}{n}, \cdots, \frac{1}{n}\right.$ ) (see the proof of Theorem 7 in Appendix 5.7.4 for details). And the corresponding expected social welfare is

$$
W_{\infty}(X)=-\frac{1}{n} \sum_{i=1}^{n} \mathbb{E}_{i, t}\left[\left(a_{i \infty}^{*}-X\right)^{2}\right]=-\frac{1}{n} \sum_{i=1}^{n} \operatorname{var}_{i, \infty}=-\frac{\sigma^{2}}{1+\sigma^{2}}
$$

The Price of Rationality ( $P o R$ ) defined as the ratio of expected social welfare under rational learning to the expected social welfare under bounded-rational learning, can then be used to analyze how the efficiency of information aggregation (or learning in general) degrades due to bounded rationality of the agents. From (5.5.2) and (5.5.4) we thus have,

$$
\text { PoR }=\frac{1+\sigma^{2}}{n+\sigma^{2}}
$$

Note that since the expected social welfare is the sum of the expected loss or cost, its possible maximum value is zero. It follows that the minimum possible value for the PoR is zero and its maximum is one. When PoR is one, the two learning mechanisms measure equally and rationality plays no role in the individual optimal decisions. If on the other hand PoR is zero, then rational learning is infinitely more efficient in aggregating information than bounded rational learning. From (5.5.5), PoR tends to zero with large $n$, implying that rationality becomes more and more 
important in efficient aggregation of information as the population size increases. So long as $\delta=1$ and agents do not find it costly to wait until all private information has been exchanged before taking action, then the communication network does not matter and only the population size does matter. If on the other hand $\delta<1$, the network topology starts to matter through its role in determining the convergence rate. The convergence rate becomes critical since agents have to optimize when to exit the game and if the convergence rate is high such that some or all agents exit the game before all the private information has been exchanged, then the actions they take at the end of the learning process will lead to higher expected loss hence lower expected social welfare. To formalize this argument, we need to formally define correct asymptotic learning in terms of actions taken at the end of the learning process. In this particular decision problem, correct asymptotic learning coincide with correct asymptotic public beliefs, but this does not always have to be the case.

DEFINITION 17: Given a sequence of networks $\{G(n)\}_{n \geq 2}$, correct asymptotic learning is said to occur if

$$
\lim _{n \rightarrow \infty} \lim _{t \rightarrow \infty} \mathbb{P}\left(\left|a_{i, t}(n)-\bar{\mu}\right|>\epsilon\right)=0 \quad \text { for all } i \in N
$$

where $\bar{\mu}=a_{i, \infty}(\infty)$ is the possible optimal action for all agents.

When $\delta=1$ the conditions for correct asymptotic learning is precisely those described in Theorems 6 and 7 for rational and bounded-rational learning respectively, and the convergence rate does not play much of a role in achieving correct asymptotic learning or public beliefs. If on the other hand $\delta<1$, the convergence rate plays a big role in determining whether or not correct asymptotic leaning actually occurs. This follows from the fact that when $\delta<1$ agents have to choose an optimal exit time, and this has an impact on how much of the private information gets exchanged before the learning process ends. It also implies that the topologies of the communication network that support correct asymptotic learning (and correct public beliefs) will no longer be those illustrated in Theorems 6 and 7 . The following proposition states conditions on the communication network for correct asymptotic learning to occur when $\delta<1$.

Proposition 14: Let $\{G(n)\}_{n \geq 2}$ be a sequence of connected communication networks, and that let $\delta<1$. If $\{D(G(n))\}_{n \geq 2}$ is the corresponding sequence of networks diameters, then correct asymptotic learning obtains under rational learning if

$$
\lim _{n \rightarrow \infty} \frac{1}{n} D(G(n))=0
$$

Under bounded-rational learning, the network must be complete. 
Proof. See Appendix 5.7.8

Proposition 14 basically emphasizes the importance of the topology of the communication network in determining the convergence rate hence efficient information aggregation, and can be summarized as follows. When $\delta<1$, though waiting reduces the expected loss associated with a mismatch between ones action and the true state of nature, waiting for too long on the other hand becomes costly as prescribed by the factor $1 / \delta$. It is therefore important that the convergence rate be high if correct learning is to ever be achieved. Condition (5.5.7) is a necessary condition for both fast and correct aggregation of information, hence correct asymptotic learning.

The network topology depicted in Figure 14 for example in which $D(G(n))=2$ for all $n$, satisfies condition (5.5.7). The network topology in Figure 13 on the other hand does not satisfy condition (5.5.7), since $D(G(n))=n$. In the case of random graphs the diameter is generally an increasing function of $n$ (see for example Bollobás [21] for analysis on the diameter of random graphs), hence correct asymptotic learning does not obtain in random networks when $\delta<1$.

The failure by agents to account for informational externalities under bounded rational learning is even more pronounced when $\delta<1$. All agents would prefer to take an action in the action space $A$ in the first or second periods since waiting longer does not improve their confidence in their beliefs.

We conclude this section by noting the indirect implication of Proposition 14 . When the communication network has a large diameter and that agents find it costly to wait before taking an action, even if agents start with a common prior, heterogeneity in public beliefs and actions will obtain.

\subsection{CONCLUSION}

Individual beliefs play a significant in determining the success of policies, initiatives and theories, and play a significant role in determining outcomes in decisions under uncertainty. Contrary to prediction from models of rationality and common prior assumptions that learning through deliberation leads to a consensus in beliefs, heterogeneity in beliefs is more of a rule than an exception in most economic and social environments. In this Chapter, we investigated the properties of beliefs resulting from rational and bounded-rational learning in social networks. We established conditions under which heterogeneity prevails, and under which such beliefs correctly aggregate private information. Under bounded-rational learning, correctness in public beliefs is determined mainly by the topology of the communication network. Specifically, for a finite population the network must be balanced and for large 
population it must be asymptotically balanced. These conditions are quite restrictive given that the topologies assumed by real world networks do not necessarily comprise of such properties. In addition to restrictions on the network topology, we also find that under bounded-rational learning, public beliefs are asymptotically correct if and only if the prior beliefs are correlated to the true state of nature.

In section 5.5 we provide a "toy" model of decision making under uncertainty, in which agents find it costly to wait much longer before taking an action. It highlights another possible source of heterogeneity in public beliefs that is not necessarily a result of historical factors or of observability of prior beliefs, but rather resulting from the topology of the communication network. That is, under such conditions heterogeneity in public beliefs will arise if the communication network has a large diameter.

Through out the Chapter we assumed that agents start with homogeneous confidence in their prior beliefs. Relaxing this assumption would give a richer understanding of the nature of public beliefs and distribution of actions in exit games or observed levels of diversity in public opinions across the population even when agents are exposed to the same sources of information (e.g Chamley and Gale [27] and Murto and Välimäki [81]). But this analysis requires a more detailed model (than the one provided in section 5.5) with specific preference structure, and we postpone this question for future research.

In conclusion, since heterogeneity in beliefs is rather a ubiquitous phenomenon in social and economic environments, one could argue that perhaps agents really do incorporate new information into their beliefs through Bayesian rational inference. If that is not the case then it could be that individuals are not as "gullible" with every new information they receive as one would like to think, and are rather more "inward-looking" when updating their beliefs. That is if agents attach more weight to their own beliefs than others' beliefs, or to beliefs of members of the subgroup they belong to than beliefs of members in other subgroups (which in both cases implies a slow speed of learning), then the observed heterogeneity in beliefs would imply that a long-run consensus prescribed by bounded-rational learning has not been reached. Or perhaps the observed nature of public beliefs indicates that individuals are heterogeneous with respect to the learning mechanisms. 


\subsection{APPENDIX}

\subsubsection{Proof of Theorem 5}

(i) If $X, \varepsilon_{1}, \cdots, \varepsilon_{n}$ are independent and it is common knowledge that $\varepsilon_{1} \sim \mathcal{N}\left(0, \sigma^{2}\right)$, then from Lemma 9

$$
\mu_{i, 1}=\frac{\sigma^{2}}{1+\sigma^{2}} \mu_{i, 0}+\frac{1}{1+\sigma^{2}} s_{i}, \quad \operatorname{var}_{i, 1}=\frac{\sigma^{2}}{1+\sigma^{2}} \quad \text { for all } i \in N
$$

We assume that under rational learning $g_{i j}=1$ if $j$ communicates to $i$ and zero otherwise. Let $G$ be static and common knowledge, and let $j_{t}$ be the index for the $t$ order neighbors. Consider any $i \in N$ with first-order degree $k_{i, 1}$. After the first round of announcements, such an $i$ updates his beliefs to a normal distribution mean

$$
\mu_{i, 2}=\frac{\sigma^{2}}{1+k_{i, 1}+\sigma^{2}} \mu_{i, 0}+\frac{1}{1+k_{i, 1}+\sigma^{2}}\left(s_{i}+\sum_{j_{1} \in N_{i, 1}} s_{j_{1}}\right) \quad \operatorname{var}_{i, 2}=\frac{\sigma^{2}}{1+k_{i, 1}+\sigma^{2}}
$$

where $s_{j_{1}}=\left(1+\sigma^{2}\right) \mu_{j_{1}, 1}-\sigma^{2} \mu_{j_{1}, 0}$ for all $j_{1} \in N_{i, 1}$. If $\mu_{j_{1}, 0}$ for all $j_{1} \in N_{i, 1}$ are observable to $i$ then $s_{j_{1}}$ 's are accurately deduced, otherwise $\mu_{j_{1}, 0}$ is simply $i$ 's ex-ante belief about the prior distribution of $j_{1}$.

Similarly, for all $j_{1} \in N_{i, 1}$ and all $l_{1} \in N_{j_{1}, 1}$ we have

$$
\mu_{j_{1}, 2}=\frac{\sigma^{2}}{1+k_{j_{1}, 1}+\sigma^{2}} \mu_{j_{1}, 0}+\frac{1}{1+k_{j_{1}, 1}+\sigma^{2}}\left(s_{j_{1}}+\sum_{l_{1} \in N_{j_{1}, 1}} s_{l_{1}}\right)
$$

and $\operatorname{var}_{j_{1}, 2}=\frac{\sigma^{2}}{1+k_{j_{1}, 1}+\sigma^{2}}$.

Since $i$ knows $k_{j_{1}, 1}$ for all $j_{1} \in N_{i, 1}$, hence $N_{i, 2}$ and $k_{i, 2}$, he can deduce each $\sum_{l_{1} \in N_{j_{1}, 1}} s_{l_{1}}$ from the first and second period announcements of all $j_{1} \in N_{i, 1}$. The posterior belief of $i$ in the third period is thus normally distributed with mean

$$
\mu_{i, 3}=\frac{\sigma^{2}}{1+k_{i, 1}+k_{i, 2}+\sigma^{2}} \mu_{i, 0}+\frac{1}{1+k_{i, 1}+k_{i, 2}+\sigma^{2}}\left(s_{i}+\sum_{j_{1} \in N_{i, 1}} s_{j_{1}}+\sum_{j_{2} \in N_{i, 2}} s_{j_{2}}\right)
$$

and variance

$$
\operatorname{var}_{i, 3}=\frac{\sigma^{2}}{1+k_{i, 1}+k_{i, 2}+\sigma^{2}}
$$

For a finite network, there exists a bound on the order of the neighborhood for all agents. That is there exists a $T_{i}$ for all $i \in N$ such that for any $t>T_{i}, N_{i, t}=0$, hence $i$ stops updating his beliefs after $t=T_{i}$. It then follows that $T_{m}=\max \left\{T_{i} ; i \in N\right\}$, which is also the diameter of the communication network, is the period after which 
learning stops for all agents. After $T_{m}$ no agents has new information to learn from his neighbors. The iteration of posterior beliefs in a manner described above leads to public belief of each $i \in N$ to be normally distributed with the mean and variance of the form

$$
\mu_{i, \infty}=\frac{\sigma^{2}}{\sum_{t=0}^{T_{m}} k_{i, t}+\sigma^{2}} \mu_{i, 0}+\frac{1}{\sum_{t=0}^{T_{m}} k_{i, t}+\sigma^{2}} \sum_{t=0}^{T_{m}} \sum_{j_{t} \in N_{i, t}} s_{j_{t}} \quad \operatorname{var}_{i, \infty}=\frac{\sigma^{2}}{\sum_{t=0}^{T_{m}} k_{i, t}+\sigma^{2}}
$$

where $s_{j_{0}}=s_{i}$ and $k_{i, 0}=1$, and that $\sum_{t=0}^{T_{m}} k_{i, t}=n, \quad \sum_{t=0}^{T_{m}} \sum_{j_{t} \in N_{i, t}} s_{j_{t}}=\sum_{j=1}^{n} s_{j}$.

(ii) Let agents' prior beliefs be uncorrelated and that $\mu_{0} \sim \mathcal{N}\left(\nu, \eta^{2} \mathbf{I}\right)$, where $\mu_{0}$ is a column vector of all $\mu_{i, 0}$ and $\mathbf{I}$ is an $n \times n$ identity matrix. Assume also that agents know the distribution of their neighbors prior beliefs but do not observe the realized value. Let $\mathbb{E}_{i}\left[s_{k} \mid v_{j}\right]$ for each $j \in N_{i, 1}$ denote the expected signal of $k$ according to $i$ given the distribution of his neighbor $j$ 's prior belief. It follows from Lemma 9 that, after observing his neighbors first period announcements, $i$ deduces the expected signals of each $j \in N_{i, 1}$ to be

$$
\begin{aligned}
\mathbb{E}_{i}\left[s_{j} \mid v_{j}\right] & =\left(1+\sigma^{2}\right) \mu_{j, 1}-\sigma^{2} v_{j} \\
& =\sigma^{2}\left(\mu_{j, 0}-v_{j}\right)+s_{j}
\end{aligned}
$$

where the second equality result from substituting for $\mu_{j, 1}$. The corresponding variance of the expected signal according to $i$ is $\operatorname{var}\left[\mathbb{E}_{i}\left[s_{j} \mid v_{j}\right]\right]=\sigma^{4} \eta^{2}+\sigma^{2}$.

After incorporating the expected signals from his neighbors, each $i \in N$ updates his beliefs to a normal distribution with mean and variance

$$
\mu_{i, 2}=\frac{\left(1+\sigma^{2}\right)\left(1+\eta^{2} \sigma^{2}\right)}{k_{i}+\left(1+\sigma^{2}\right)\left(1+\eta^{2} \sigma^{2}\right)} \mu_{i, 1}+\frac{1}{k_{i}+\left(1+\sigma^{2}\right)\left(1+\eta^{2} \sigma^{2}\right)} \sum_{j \in N_{i, 1}} \mathbb{E}_{i}\left[s_{j} \mid v_{j}\right]
$$

and

$$
\operatorname{var}_{i, 2}=\frac{\sigma^{2}\left(1+\eta^{2} \sigma^{2}\right)}{k_{i}+\left(1+\sigma^{2}\right)\left(1+\eta^{2} \sigma^{2}\right)}
$$

Let $l$ be the index for the neighbors of $j \in N_{i, 1}$. We maintain the assumption that agents have memory of past announcements of their neighbors and that the network is common knowledge. From the second period announcements of his neighbors, $i$ deduces the new information from each $j \in N_{i, 1}$ to be

$$
\sum_{l \in N_{j, 1}} \mathbb{E}_{i}\left[s_{l} \mid v_{j}\right]=\left(k_{j}+\left(1+\sigma^{2}\right)\left(1+\eta^{2} \sigma^{2}\right)\right) \mu_{j, 2}-\left(1+\sigma^{2}\right)\left(1+\eta^{2} \sigma^{2}\right) \mu_{j, 1}
$$


where each $\mathbb{E}_{i}\left[s_{l} \mid v_{j}\right]$ is of the form $\mathbb{E}_{i}\left[s_{l} \mid v_{j}\right]=\sigma^{2}\left(\mu_{l, 0}-v_{l}\right)+s_{l}$. At the end of the third period, $i$ updates his beliefs to a normal distribution with mean and variance

$$
\begin{aligned}
\mu_{i, 3} & =\frac{\left(1+\sigma^{2}\right)\left(1+\eta^{2} \sigma^{2}\right)}{k_{i}+k_{i, 2}+\left(1+\sigma^{2}\right)\left(1+\eta^{2} \sigma^{2}\right)} \mu_{i, 1} \\
& +\frac{1}{k_{i}+k_{i, 2}+\left(1+\sigma^{2}\right)\left(1+\eta^{2} \sigma^{2}\right)}\left(\sum_{j \in N_{i, 1}} \mathbb{E}_{i}\left[s_{j} \mid v_{j}\right]+\sum_{j \in N_{i, 1}} \sum_{l \in N_{j, 1}} \mathbb{E}_{i}\left[s_{l} \mid v_{j}\right]\right)
\end{aligned}
$$

and

$$
\operatorname{var}_{i, 3}=\frac{\sigma^{2}\left(1+\eta^{2} \sigma^{2}\right)}{k_{i}+\sum_{j \in N_{i, 1}} k_{j}+\left(1+\sigma^{2}\right)\left(1+\eta^{2} \sigma^{2}\right)}
$$

By iterating the posterior beliefs until the end of the learning process and noting that $\sum_{t=0}^{T_{m}} k_{i, t}=n$, the public belief of each $i \in N$ is then normally distributed with mean and variance

$$
\mu_{i, \infty}=\frac{\left(1+\sigma^{2}\right)\left(1+\eta^{2} \sigma^{2}\right)}{\left(1+\sigma^{2}\right)\left(1+\eta^{2} \sigma^{2}\right)+n-1} \mu_{i, 1}+\frac{1}{\left(1+\sigma^{2}\right)\left(1+\eta^{2} \sigma^{2}\right)+n-1} \sum_{l \in N \backslash\{i\}} \mathbb{E}_{i}\left[s_{l} \mid \boldsymbol{v}\right]
$$

and

$$
\operatorname{var}_{i, \infty}=\frac{\sigma^{2}\left(1+\eta^{2} \sigma^{2}\right)}{\left(1+\sigma^{2}\right)\left(1+\eta^{2} \sigma^{2}\right)+n-1}
$$

(iii) In Theorem 5 (iii) we assume that $\mu_{0} \sim \mathcal{N}(v, M)$, where each element of $M$, $m_{i j}>0$ for all $(i, j) \in N$.

The following notations will be used in the proof. We write $\boldsymbol{\mu}$ and $\boldsymbol{\mu}_{-i}$ for the column vectors of all $\mu_{i}$ for all $i \in N$ and for all $i \in N \backslash\{i\}$ respectively. Similarly, $\mu$ and $v_{-i}$ denote the column vectors of all $v_{i}$ for all $i \in N$ and for all $i \in N \backslash$ $\{i\}$ respectively. $M_{-i,-i}$ denotes and $n-1 \times n-1$ variance-covariance matrix of all agents excluding $i$, and $M_{-i, i}$ is the $i$ th column of $M$ with the $i$ th row excluded. We also denote by $\mathbf{1}_{k \times l}$ for a $k \times l$ dimensional matrix of ones, and by $\mathbf{I}$ the identity matrix.

We employ the following well known concepts for normally distributed random variables and specifically adopted to the distributions of prior beliefs of agents. Let $\mu_{0}$ be the column vector of prior beliefs of all agents, $\mu_{i, 0} \forall i \in N$ and $\mu_{-i, 0} \forall i \in N \backslash\{i\}$. If $\mu_{0} \sim \mathcal{N}(\nu, M)$, then

$$
\begin{aligned}
& \mathbb{E}\left[\boldsymbol{\mu}_{-i, 0} \mid \mu_{i, 0}\right]=\boldsymbol{\nu}_{-i}+m_{i i}^{-1} M_{-i, i}\left(\mu_{i, 0}-v_{i}\right) \\
& \operatorname{var}\left[\boldsymbol{\mu}_{-i, 0} \mid \mu_{i, 0}\right]=M_{-i,-i}-m_{i i}^{-1} M_{-i, i} M_{-i, i}^{\prime}
\end{aligned}
$$

When agents priors are correlated, the expected signal of each $j \in N_{i, 1}$ according to $i$ given the realization $\mu_{i, 0}$ and $j$ 's first period announcement $\mu_{j, 1}$ is given by

$$
\mathbb{E}_{i}\left[s_{j} \mid \mu_{i, 0}, \mu_{j, 1}\right]=\left(1+\sigma^{2}\right) \mu_{j, 1}-\sigma^{2} \mathbb{E}_{i}\left[\mu_{j, 0} \mid \mu_{i, 0}\right]
$$


In a similar manner, we can write the joint expected signals for all $j \in N_{i, 1}$ according to an agent $i$ who observes the announcements of all other agents as follows

$$
\mathbb{E}_{i}\left[\mathbf{s}_{-i} \mid \mu_{i, 0}, \boldsymbol{\mu}_{-i, 1}\right]=\left(1+\sigma^{2}\right) \boldsymbol{\mu}_{-i, 1}-\sigma^{2} \mathbb{E}_{i}\left[\boldsymbol{\mu}_{-i, 0} \mid \mu_{i, 0}\right]
$$

which by substituting for $\boldsymbol{\mu}_{-i, 1}$ is equivalent to

$$
\mathbb{E}_{i}\left[\mathbf{s}_{-i} \mid \mu_{i, 0}, \boldsymbol{\mu}_{-i, 1}\right]=\sigma^{2}\left(\boldsymbol{\mu}_{-i, 0}-\mathbb{E}_{i}\left[\boldsymbol{\mu}_{-i, 0} \mid \mu_{i, 0}\right]\right)+\mathbf{s}_{-i}
$$

The variance associated with the expected signal is then

$$
\begin{aligned}
\operatorname{var}\left[\mathbf{s}_{-i} \mid \mu_{i, 0}, \boldsymbol{\mu}_{-i, 1}\right] & =\sigma^{4} \operatorname{var}\left[\boldsymbol{\mu}_{-i, 0} \mid \mu_{i, 0}\right]+\sigma^{2} \mathbf{I} \\
& =\sigma^{4}\left(M_{-i,-i}-m_{i i}^{-1} M_{-i, i} M_{-i, i}^{\prime}\right)+\sigma^{2} \mathbf{I}
\end{aligned}
$$

We now derive the coefficient of $\mathbb{E}_{i}\left[\mathbf{s}_{-i} \mid \mu_{i, 0}, \mu_{-i, 1}\right]$ in the second period announcement of $i$. Note that the variance associated with $\mu_{i, 1}$ is $\frac{\sigma^{2}}{1+\sigma^{2}}$, and together with $\operatorname{var}\left[\mathbf{s}_{-i} \mid \mu_{i, 0}, \boldsymbol{\mu}_{-i, 1}\right]$ we can define an $n-1 \times n-1$ matrix $C$ as follows

$$
\begin{aligned}
C & =\frac{\mathbf{1}_{n-1 \times n-1}}{\left(\frac{\sigma^{2}}{1+\sigma^{2}}\right) \mathbf{1}_{n-1 \times n-1}+\operatorname{var}\left[\mathbf{s}_{-i} \mid \mu_{i, 0}, \boldsymbol{\mu}_{-i, 1}\right]} \\
& =\frac{\left(1+\sigma^{2}\right) \mathbf{1}_{n-1 \times n-1}}{\sigma^{2} \mathbf{1}_{n-1 \times n-1}+\sigma^{2}\left(1+\sigma^{2}\right)\left(\sigma^{2}\left(M_{-i,-i}-m_{i i}^{-1} M_{-i, i} M_{-i, i}^{\prime}\right)+\mathbf{I}\right)}
\end{aligned}
$$

Let $C_{i}$ denote the $i$ th row of $C$, that is

$$
C_{i}=\left(1+\sigma^{2}\right) \mathbf{1}_{1 \times n-1}\left[\sigma^{2} \mathbf{1}_{n-1 \times n-1}+\sigma^{2}\left(1+\sigma^{2}\right)\left(\sigma^{2}\left(M_{-i,-i}-m_{i i}^{-1} M_{-i, i} M_{-i, i}^{\prime}\right)+\mathbf{I}\right)\right]^{-1}
$$

The elements of $C_{i}$ are the coefficients associated with the expected signals of all $j \in N \backslash\{i\}$ in the second period announcement of $i$. We thus have

$$
\begin{aligned}
\mu_{i, 2} & =\left(1-C_{i} \mathbf{1}_{1 \times n-1}\right) \mu_{i, 1}+C_{i} \mathbb{E}_{i}\left[\mathbf{s}_{-i} \mid \mu_{i, 0}, \boldsymbol{\mu}_{-i, 1}\right] \\
& =\left(1-C_{i} \mathbf{1}_{1 \times n-1}\right) \mu_{i, 1}+\left(1+\sigma^{2}\right) C_{i} \boldsymbol{\mu}_{-i, 1}-\sigma^{2} C_{i} v_{-i}-\sigma^{2} m_{i i}^{-1} C_{i} M_{-i, i}\left(\mu_{i, 0}-v_{i}\right)
\end{aligned}
$$

From the second period announcement of $i$, all agents who observe $i$ 's announcements know that

$$
\mu_{i, 0}=v_{i}+\frac{\left(1-C_{i} \mathbf{1}_{1 \times n-1}\right) \mu_{i, 1}+\left(1+\sigma^{2}\right) C_{i} \boldsymbol{\mu}_{-i, 1}-\sigma^{2} C_{i} \boldsymbol{v}_{-i}-\mu_{i, 2}}{\sigma^{2} m_{i i}^{-1} C_{i} M_{-i, i}}
$$

From (5.7.1.13), each agent who communicates to $i$ and $i$ communicates to, will correctly deduce $\mu_{i, 0}$ at the end of the second period announcements if and only if that 
agent also observes the announcements of all other agents that communicate to $i$ and $i$ communicates to. This follows from the fact that for any $j \in N_{i, 1}$ to correctly deduce $\mu_{i, 0}$, then $j$ must also observe all $\mu_{k, 1} \in \mu_{-i, 1}$ for $k \neq j$. The conclusion is that the communication network must be complete if agents are to correctly deduce the neighbors' realized prior beliefs and hence private information. That is, from the second period announcements, all agents (who observes $i$ 's announcements) deduces that

$$
s_{i}=\left(1+\sigma^{2}\right) \mu_{i, 1}-\sigma^{2}\left(v_{i}+\frac{\left(1-C_{i} \mathbf{1}_{1 \times n-1}\right) \mu_{i, 1}+\left(1+\sigma^{2}\right) C_{i} \boldsymbol{\mu}_{-i, 1}-\sigma^{2} C_{i} \boldsymbol{v}_{-i}-\mu_{i, 2}}{\sigma^{2} m_{i i}^{-1} C_{i} M_{-i, i}}\right)
$$

Since the network is complete, the third period announcement of each $i \in N$ a normal distribution with mean

$$
\mu_{i, \infty}(n)=\frac{\sigma^{2}}{n+\sigma^{2}} \mu_{i, 0}+\frac{1}{n+\sigma^{2}} \sum_{j=0}^{n} s_{j}
$$

\subsubsection{Proof of Proposition 11}

To proof the proposition, we first define the notion coefficient of ergodicity as a measure for ergodicity [101].

Definition 18: Given a matrix $G$ with entries $g_{i j}$, the coefficient of ergodicity $\rho(G)$ defined on the $L^{1}$-norm of $G$ is

$$
\rho(G)=\frac{1}{2}\left\{\max _{i, j} \sum_{k=1}^{n}\left(\left|g_{i k}-g_{j k}\right|\right)\right\},
$$

with the following properties;

(i) $0 \leq \rho(G) \leq 1$.

(ii) For two matrices $G_{1}$ and $G_{2}, \rho\left(G_{1} G_{2}\right) \leq \rho\left(G_{2}\right) \rho\left(G_{2}\right)$.

(iii) $\rho(G)=0$ if and only if $\operatorname{rank}(G)=1$; that is $G=\mathbf{e v}^{\prime}$

Let $G^{T}=\prod_{t=0}^{T} G_{\gamma(t)}$, then property $(i i)$ of Definition 12 implies that

$$
\rho\left(G^{T}\right) \leq \rho\left(G_{\gamma(T)}\right) \rho\left(G_{\gamma(T-1)}\right) \cdots \rho\left(G_{\gamma(0)}\right)
$$

Property (iii) together with Lemma 12 imply that ergodic Markov chains are those in which

$$
\lim _{T \rightarrow \infty} \rho\left(G^{T}\right)=0
$$


We can thus deduce from properties (ii) and (iii) of Definition 12, specially equations (5.7.2.2) and (5.7.2.3) that a chain is ergodic if there exists a $\tau \geq 0$ such that for every time interval $[t, t+\tau)$ there exists a sequence of matrices $\left\{G_{\gamma(t)}, \cdots, G_{\gamma(t+\tau)}\right\}$, for which the coefficient of ergodicity of their product is less than unity. That is $\rho\left(G_{\gamma(t)} \cdots G_{\gamma(t+\tau)}\right)<1$. Matrices or a product of matrices for which the coefficient of ergodicity is less than unity are known as scrambling matrices. The question we then ask is, what switching strategy induces a sequence of networks whose product on intervals of time result into scrambling matrices? The following lemmas establish the necessary conditions.

LeMMA 13: Let $G_{1}$ and $G_{2}$ be two network induced transition matrices, and that both matrices are aperiodic. If $G_{1}$ is connected, then the products $G_{1} G_{2}$ and $G_{2} G_{1}$ are also connected and aperiodic.

Proof. Write $G_{2}$ in the form $G_{2}=\operatorname{Diag}\left(G_{2}\right)+G_{2}^{\prime}$, where $\operatorname{Diag}\left(G_{2}\right)$ is a diagonal matrix whose elements are the diagonal of $G_{2}$, and $G_{2}^{\prime}$ is the residual $G_{2}-\operatorname{Diag}\left(G_{2}\right)$. Since $G_{2}$ is non-negative then so is $G_{2}^{\prime}$. We thus have that

$$
G_{2} G_{1}=\operatorname{Diag}\left(G_{2}\right) G_{1}+G_{2}^{\prime} G_{1}
$$

Denote by $g_{i i}^{2}$ and $g_{i j}^{1}$ for $1 \leq i \leq n$ as the elements of $\operatorname{Diag}\left(G_{2}\right)$ and $G_{1}$ respectively. Then

$$
\operatorname{Diag}\left(G_{2}\right) G_{1}=\left(\begin{array}{ccc}
g_{11}^{2} g_{11}^{1} & \cdots & g_{11}^{2} g_{1 n}^{1} \\
\vdots & & \vdots \\
g_{n n}^{2} g_{n 1}^{1} & \cdots & g_{n n}^{2} g_{n n}^{1}
\end{array}\right)
$$

Clearly if $G_{1}$ is connected and aperiodic, then so is $\operatorname{Diag}\left(G_{2}\right) G_{1}$. The matrix product is non-negative since $G_{1}$ is also non-negative, such that when added to $\operatorname{Diag}\left(G_{2}\right) G_{1}$ the properties of connectedness aperiodicity are preserved. A similar argument follows for $G_{1} G_{2}$.

LeMMA 14: Let $\left\{G_{\gamma(t)}\right\}_{t \geq 0}$ be a sequence of connected aperiodic transition matrices, then there exists a sufficiently large $\tau>0$ such that

$$
G^{\tau}=G_{\gamma(t)} \cdots G_{\gamma(t+\tau)}
$$

is a scrambling matrix.

Proof. The proof follows from the fact that a network that is connected and aperiodic induces a primitive transition matrix. That is let $G$ be such a matrix, then there exists a sufficiently large $t$ such that $G^{t}$ is a positive matrix. Since positive stochastic matrices are scrambling, it follows that the product of a sequence of connected aperiodic matrices is scrambling. 
The following lemma then directly follows from the above two

LEMMA 15: Let $T_{2}<T_{1}<T$ be sufficiently large integers. If $\gamma$ is such that there exist a $T_{2} \geq 0$ and $T_{1} \geq 0$ where by for every time interval $\left[t, t+T_{2}\right)$ a connected communication network obtains, such that within the time interval $\left[t, t+T_{1}\right)$ a scrambling connected network obtains, then

$$
\lim _{T \rightarrow \infty} \prod_{t=0}^{T} G_{\gamma(t)}=\mathbf{e v}^{\prime}
$$

The expression on the right hand side of (5.3.6) then follows directly from Lemma 15

\subsubsection{Proof of Theorem 6}

The general assumption in all the proofs below is that the sequence $\{G(n)\}_{n \geq 2}$ is common knowledge

(i) There are two parts to the proof of Theorem $6(i)$; when the prior beliefs are observable and when they are not. We first prove for the case in which the realized prior beliefs are observable, that is for all $i \in N$, each $\mu_{j, 0}$ for all $j \in N_{i, 1}$ is observable to $i$. Under this assumption, the public belief of each $i$ is normally distributed with mean

$$
\mu_{i, \infty}(n)=\frac{\sigma^{2}}{n+\sigma^{2}} \mu_{i, 0}+\frac{1}{n+\sigma^{2}} \sum_{j=0}^{n} s_{j}
$$

The variance of $\mu_{i, \infty}(n)$ is then

$$
\operatorname{var}\left[\mu_{i, \infty}(n)\right]=\frac{\sigma^{4} \eta^{2}}{\left(n+\sigma^{2}\right)^{2}}+\frac{n \sigma^{2}}{\left(n+\sigma^{2}\right)^{2}}
$$

From Chernoff bound, it follows that

$$
\begin{aligned}
\left.\lim _{n \rightarrow \infty} \mathbb{P}\left(\left|\mu_{i, \infty}(n)-\bar{\mu}\right|>\epsilon\right)\right) & \leq \lim _{n \rightarrow \infty}\left(\frac{\operatorname{var}\left[\mu_{i, \infty}(n)\right]}{\epsilon}\right) \\
& =\lim _{n \rightarrow \infty}\left(\frac{\sigma^{4} \eta^{2}}{\left(n+\sigma^{2}\right)^{2}}+\frac{n \sigma^{2}}{\left(n+\sigma^{2}\right)^{2}}\right)=0
\end{aligned}
$$

It is easy to see that the right hand side of (5.7.3.2) will be zero if and only if both $\sigma$ and $\eta$ are finite such that $\sigma^{4} \eta^{2}<\infty$. This conditions applies to all $i \in N$ The limit of the variance of $X$ is

$$
\lim _{n \rightarrow \infty} \operatorname{var}_{i, \infty}(n)=\lim _{n \rightarrow \infty} \frac{\sigma^{2}}{n+\sigma^{2}}=0 .
$$


Now we prove for the case in which the realized priors are unobservable. From Theorem 5, if for each $i \in N, \mu_{j, 0}$ for all $j \in N_{i, 1}$ are unobservable but it is common knowledge that $\mu_{0} \sim \mathcal{N}\left(v, \eta^{2} \mathbf{I}\right)$, where $\mu_{0}$ is a column vector of all $\mu_{i, 0}$, then the public belief of each $i$ is normally distributed with mean

$\mu_{i, \infty}(n)=\frac{\left(1+\sigma^{2}\right)\left(1+\eta^{2} \sigma^{2}\right)}{\left(1+\sigma^{2}\right)\left(1+\eta^{2} \sigma^{2}\right)+n-1} \mu_{i, 1}+\frac{1}{\left(1+\sigma^{2}\right)\left(1+\eta^{2} \sigma^{2}\right)+n-1} \sum_{l \in N \backslash\{i\}} \mathbb{E}_{i}\left[s_{l} \mid \boldsymbol{v}\right]$

For the sake of notational convenience, let $\varphi=\left(1+\sigma^{2}\right)\left(1+\eta^{2} \sigma^{2}\right)$, and recall that $\mathbb{E}_{i}\left[s_{l} \mid v\right]=\sigma^{2}\left(\mu_{l, 0}-v_{l}\right)+s_{l}$ for all $i \in N$ and all $l \in N \backslash\{i\}$. We can then rewrite $(5 \cdot 7 \cdot 3 \cdot 3)$ as

$$
\mu_{i, \infty}(n)=\frac{\varphi}{\varphi+n-1} \mu_{i, 1}+\frac{\sigma^{2}}{\varphi+n-1} \sum_{l \in N \backslash\{i\}}\left(\mu_{l, 0}-v_{l}\right)+\frac{1}{\varphi+n-1} \sum_{l \in N \backslash\{i\}} s_{l}
$$

The variance associated with $\mu_{i, \infty}(n)$ in $(5 \cdot 7 \cdot 3 \cdot 4)$ is

$$
\operatorname{var}\left[\mu_{i, \infty}(n)\right]=\frac{\varphi^{2} \sigma^{4} \eta^{2}+\varphi^{2} \sigma^{2}}{(\varphi+n-1)^{2}\left(1+\sigma^{2}\right)^{2}}+\frac{\sigma^{4} \eta^{2}}{(\varphi+n-1)^{2}}+\frac{(n-1) \sigma^{2}}{(\varphi+n-1)^{2}}
$$

Similarly from $(5 \cdot 7 \cdot 3 \cdot 5)$, the $\lim _{n \rightarrow \infty} \operatorname{var}\left[\mu_{i, \infty}(n)\right]=0$ if and only if $\eta$ and $\sigma$ are finite. Note that the sequence of random variables $\left\{\left(\mu_{l, 0}-v_{l}\right)\right\}_{l \in N \backslash\{i\}}$ is of mean zero and variance $\eta^{2}$. This implies that if each term in the sum on the right hand side of (5.7.3.4) converges in probability, then the first and the second term both converge to zero and the third term converges to $\bar{\mu}$. It then follows from $(5 \cdot 7 \cdot 3.2)$ that $\mu_{i, \infty}(n) \stackrel{p}{\rightarrow} \bar{\mu}$.

If $\eta$ and $\sigma$ are finite, then limit of the variance of $X$ when priors beliefs are not observable is

$$
\lim _{n \rightarrow \infty} \frac{\sigma^{2}\left(1+\eta^{2} \sigma^{2}\right)}{\left(1+\sigma^{2}\right)\left(1+\eta^{2} \sigma^{2}\right)+n-1}=0 .
$$

(ii) From (5.7.3.2) it follows that when the conditions of Theorem 6 (ii) are satisfied, $\mu_{i, \infty}(n) \stackrel{p}{\rightarrow} \bar{\mu}$.

\subsubsection{Proof of Theorem 7}

(i) Consider the case in which $G(n)$ is doubly stochastic such that $\sum_{j=1}^{n} g_{i j}(n)=$ $\sum_{i=1}^{n} g_{i j}(n)$. Let $\mathbf{z}$ and $\mathbf{y}$ be the left and right eigenvalues of $G(n)$ associated with the leading eigenvalue. If $G(n)$ is doubly stochastic then $\mathbf{z}=\mathbf{y}$, such that

$$
\mathbf{z}^{\prime} G(n)=\mathbf{z}^{\prime} \quad \text { and } \quad G(n) \mathbf{z}=\mathbf{z}
$$


Without loss of generality we can assume that $\|\mathbf{z}\|=\mathbf{z}^{\prime} \mathbf{z}=1$. Specifically, $\mathbf{z}=\frac{1}{\sqrt{N}} \mathbf{e}$, where $\mathbf{e}$ is a length $n$ vector of ones. From Lemma 12 it follows that $\mathbf{v}=\frac{1}{n} \mathbf{e}$, such that for each $i \in N$

$$
\mu_{i, \infty}(n)=\frac{1}{n} \sum_{i=1}^{n} \mu_{i, 1}
$$

(ii) Recall that

$$
\begin{aligned}
\mu_{i, \infty}(n) & =\sum_{j=1}^{n} v_{j}(n)\left(\frac{\sigma^{2}}{1+\sigma^{2}} \mu_{j, 0}+\frac{1}{1+\sigma^{2}} s_{j}\right) \\
& =\frac{1}{1+\sigma^{2}} \bar{\mu}+\frac{\sigma^{2}}{1+\sigma^{2}} \sum_{j=1}^{n} v_{j}(n) \mu_{j, 0}+\frac{\sigma^{2}}{1+\sigma^{2}} \sum_{j=1}^{n} v_{j}(n) \varepsilon_{j}
\end{aligned}
$$

where the second equality follows from the fact that $s_{j}=\bar{\mu}+\varepsilon_{j}$ and that $\sum_{j=1}^{n} v_{j}(n)=1$. Let the summation components on the right hand side of 5.7.4.1 be denoted as follows

$$
V_{\mu}(n)=\sum_{j=1}^{n} v_{j}(n) \mu_{j, 0}, \quad \text { and } \quad V_{\varepsilon}(n)=\sum_{j=1}^{n} v_{j}(n) \varepsilon_{j} .
$$

Since $\bar{\mu}$ is a parameter, it follows that any variance in $\mu_{i, \infty}(n)$ is due to the variances in $V_{\mu}(n)$ and $V_{\varepsilon}(n)$. This in turn implies that for $\mu_{i, \infty}(n)$ to converge in probability, then both $V_{\mu}(n)$ and $V_{\varepsilon}(n)$ must also converge in probability to their respective limits. Consider first the in which the communication network is balanced, in which case (5.7.4.1) becomes.

$$
\mu_{i, \infty}(n)=\frac{1}{1+\sigma^{2}} \bar{\mu}+\frac{\sigma^{2}}{1+\sigma^{2}} \frac{1}{n} \sum_{j=1}^{n} \mu_{j, 0}+\frac{\sigma^{2}}{1+\sigma^{2}} \frac{1}{n} \sum_{j=1}^{n} \varepsilon_{j}
$$

This implies that both $V_{\mu}(n)$ and $V_{\varepsilon}(n)$ are simply sample averages of the random variables $\mu_{i, 0}$ and $\varepsilon_{i}$ of all $i \in N$ respectively. It then follows from the law of large numbers that $V_{\varepsilon}(n)$ converge in probability to zero, and $V_{\mu}(n)$ converges in probability to $\bar{\mu}$ only if $\mu_{0} \sim \mathcal{N}\left(\bar{\mu}, \eta^{2} \mathbf{I}\right)$. This in turn implies that $\mu_{i, \infty}(n)$ also converges in probability to $\bar{\mu}$. That is

$$
\mu_{i, \infty}(\infty)=\frac{1}{1+\sigma^{2}} \bar{\mu}+\frac{\sigma^{2}}{1+\sigma^{2}} \bar{\mu}=\bar{\mu}
$$

Generally speaking, both $V_{\mu}(n)$ and $V_{\varepsilon}(n)$ are sample weighted averages of $n$ random variable $\mu_{i, 0}$ and $\varepsilon_{i}$ respectively drawn with probabilities $v_{i}(n)$ for 
each $i \in N$, from normal distributions with means $\bar{\mu}$ and zero variance. These sample means converge in probability if and only if their variances converge to zero for large $n$. That is

$$
\lim _{n \rightarrow \infty} \operatorname{var}\left[V_{\mu}(n)\right]=0 \quad \text { and } \quad \lim _{n \rightarrow \infty} \operatorname{var}\left[V_{\varepsilon}(n)\right]=0
$$

The respective variances are

$$
\operatorname{var}\left[V_{\mu}(n)\right]=\eta^{2} \sum_{j=1}^{n} v_{j}^{2}(n) \text { and } \operatorname{var}\left[V_{\varepsilon}(n)\right]=\sigma^{2} \sum_{j=1}^{n} v_{j}^{2}(n),
$$

which converge to zero if and only if the summand $\sum_{j=1}^{n} v_{j}^{2}(n)$ converges to zero with $n$. This sum converges to zero if each square $v_{j}^{2}(n)$ converges to zero with large $n$. In fact it is enough to say that $\max _{i \in N}\left(v_{i}(n)\right) \rightarrow 0$ for large $n$. This condition is satisfied by asymptotically balanced networks in that if $G(n)$ is asymptotically balanced, then $G(n)$ converges to doubly stochastic matrix $S$. Where for any doubly stochastic matrix, the elements of the corresponding weight vector $v_{i}(n)=\frac{1}{n}$, converge to zero for large $n$.

In the case of the variance of public belief, it follows directly that

$$
\begin{aligned}
\operatorname{var}_{i, \infty}(n) & =\sum_{j=1}^{n} v_{j}(n) \frac{\sigma}{1+\sigma} \\
& =\frac{\sigma}{1+\sigma} \sum_{j=1}^{n} v_{j}(n)=\frac{\sigma}{1+\sigma}
\end{aligned}
$$

\subsubsection{Proof of Proposition 12}

Given $G(n)$, the closest doubly stochastic matrix $S(n)$ to $G(n)$ is that which minimizes the quantity $\|S(n)-G(n)\|_{F}^{2}$. Our objective is then as follows:

$$
\begin{array}{lcl}
\min _{S(n) \in \mathcal{S}(n)} & \|S(n)-G(n)\|_{F}^{2} & \\
\text { subject to } & S(n) \mathbf{z}=\mathbf{z} & \mathbf{z}^{\prime} S(n)=\mathbf{z}^{\prime}
\end{array}
$$

where $\mathcal{S}(n)$ is the set of all doubly stochastic matrices of size $n$, and $\mathbf{z}$ is the left and right eigenvectors corresponding to the first (unit) eigenvalue of $S(n)$. We assume without loss of generality that $\|\mathbf{z}\|=\mathbf{z}^{\prime} \mathbf{z}=1$. The elements of $G(n)$ are denoted by $g_{i j}(n)$ and those for $S(n)$ by $s_{i j}$. It is easy to see that the objective function to be minimized is of the form

$$
f(G(n))=\left(s_{11}-g_{11}\right)^{2}+\cdots+\left(s_{1 n}-g_{1 n}\right)^{2}+\cdots+\left(s_{n 1}-g_{n 1}\right)^{2}+\cdots+\left(s_{n n}-g_{n n}\right)^{2}
$$


Note that the constraints together make a total of $2 n$ linear equations, which leads to $2 n$ Lagrange multipliers denoted (in vector form) by $2 \alpha=\left(2 \alpha_{1}, \cdots, 2 \alpha_{n}\right)$ and $2 \beta=\left(2 \beta_{1}, \cdots, 2 \beta_{n}\right)$. Note also that the factor of 2 in $\alpha$ and $\beta$ is to account for the factor of 2 in the derivative of $f(G(n))$ below. The Kuhn-Tucker conditions yield the following matrix equation.

$$
(S(n)-G(n))+\alpha \mathbf{z}^{\prime}+\mathbf{z} \beta^{\prime}=0
$$

By substituting for $S(n)=G(n)-\alpha \mathbf{z}^{\prime}-\mathbf{z} \beta^{\prime}$ into $S(n) \mathbf{z}=\mathbf{z}$ and $\mathbf{z}^{\prime} S(n)=\mathbf{z}^{\prime}$, together with the assumption that $\mathbf{z}^{\prime} \mathbf{z}=1$, one obtains the following set of simultaneous equations.

$$
\begin{gathered}
G(n) \mathbf{z}-\alpha-\mathbf{z} \beta^{\prime} \mathbf{z}=\mathbf{z} \\
\mathbf{z}^{\prime} G(n)-\mathbf{z}^{\prime} \alpha \mathbf{z}^{\prime}-\beta^{\prime}=\mathbf{z}^{\prime},
\end{gathered}
$$

which can be written in matrix form as

$$
\left[\begin{array}{cc}
I & \mathbf{z z}^{\prime} \\
\mathbf{z z}^{\prime} & I
\end{array}\right]\left[\begin{array}{l}
\alpha \\
\beta
\end{array}\right]=\left[\begin{array}{l}
G(n) \mathbf{z}-\mathbf{z} \\
G(n)^{\prime} \mathbf{z}-\mathbf{z}
\end{array}\right]
$$

We can then solve for $\alpha$ and $\beta$ by multiplying both sides of $(5 \cdot 7 \cdot 5 \cdot 4)$ by the inverse of $\left[\begin{array}{cc}I & \mathbf{z z}^{\prime} \\ \mathbf{z z} & I\end{array}\right]$. That is

$$
\left[\begin{array}{ll}
I & 0 \\
0 & I
\end{array}\right]\left[\begin{array}{l}
\alpha \\
\beta
\end{array}\right]=\frac{1}{1-\mathbf{z z}^{\prime}}\left[\begin{array}{cc}
I & -\mathbf{z z}^{\prime} \\
-\mathbf{z z} & I
\end{array}\right]\left[\begin{array}{c}
G(n) \mathbf{z}-\mathbf{z} \\
G^{\prime}(n) \mathbf{z}-\mathbf{z}
\end{array}\right]
$$

which yields,

$$
\alpha=G(n) \mathbf{z}-\mathbf{z} \quad \text { and } \beta=G^{\prime}(n) \mathbf{z}-\mathbf{z}
$$

The assumption that $\mathbf{z}^{\prime} \mathbf{z}=1$ also implies that $\mathbf{z}=\frac{1}{\sqrt{n}} \mathbf{e}$. Substituting for $\alpha$ and $\beta$ in (5.7.5.1) and noting that $G(n) \mathbf{e}=\mathbf{e}$, we obtain

$$
S(n)=G(n)+\frac{1}{n} \mathbf{e e}^{\prime}-\frac{1}{n} \mathbf{e e}^{\prime} G(n)
$$

It then follows that

$$
\begin{aligned}
\phi(n)^{2}=\|S(n)-G(n)\|_{F}^{2} & =\frac{1}{n^{2}}\left\|\mathbf{e e}^{\prime}-\mathbf{e e}^{\prime} G(n)\right\|_{F}^{2} \\
& =\frac{1}{n} \sum_{j=1}^{n}\left(1-\sum_{i=1}^{n} g_{i j}(n)\right)^{2} .
\end{aligned}
$$




\subsubsection{Proof of Theorem 8}

The proof follows from Proposition 12, where a network is said to be asymptotically balanced if

$$
\lim _{n \rightarrow \infty} \phi(n)=0 .
$$

For Theorem $8(i)$, we have that

$$
\begin{aligned}
\phi^{2}(n) & =\frac{1}{n} \sum_{j=1}^{n}\left(1-\sum_{i=1}^{n} g_{i j}(n)\right)^{2} \\
& =\frac{1}{n} \sum_{j=1}^{n}\left(1-2 \sum_{i=1}^{n} g_{i j}(n)+\left(\sum_{i=1}^{n} g_{i j}(n)\right)^{2}\right) \\
& =\frac{1}{n}\left(n-2 \sum_{i=1}^{n} \sum_{j=1}^{n} g_{i j}(n)+\sum_{j=1}^{n}\left(\sum_{i=1}^{n} g_{i j}(n)\right)^{2}\right) \\
& \leq \frac{1}{n}\left(d_{\max }^{i n}(n)\left(\sum_{i=1}^{n} \sum_{j=1}^{n} g_{i j}(n)\right)-n\right) \\
& =d_{\max }^{\text {in }}(n)-1
\end{aligned}
$$

such that $\lim _{n \rightarrow \infty} \phi(n)=0$ if $\lim _{n \rightarrow \infty} d_{\text {max }}^{\text {in }}(n)=1$.

For the case of Theorem $8(i i)$, since for $n>n^{\prime}$ the quantity $D(n)$ is constant say $c$, then for all $n>n^{\prime}$

$$
\phi^{2}(n)=\frac{1}{n} c
$$

In which case $\lim _{n \rightarrow \infty} \frac{1}{n} c=0$.

\subsubsection{Proof of Proposition 13}

Under Bayesian rational learning, the proof follows directly from the fact that learning stops after the two agents that form the longest geodesic (diameter of the network) have communicated their private informations (see proof of Theorem 5). The time it takes private beliefs to become public is thus $t_{c}=D\left(G_{\gamma_{c}}\right)$, in which case the convergence rate is basically $\frac{1}{t_{c}}$. The relationship between the diameter and the second largest eigenvalue of a graph is a well studied concept in graph theory (e.g Chung [28]). Generally it assumes the form,

$$
D\left(G_{\gamma_{c}}\right) \leq \frac{\ln (c)}{\ln \left(1 / \lambda_{2}\left(G_{\gamma_{c}}\right)\right)}
$$

where $c$ is some constant. 
In the case of Bayesian bounded-rational learning mechanism, it is well known that a homogeneous Markov chain with transition matrix $G_{\gamma_{c}}$ whose second largest eigenvalue is $\lambda_{2}\left(G_{\gamma_{c}}\right)$ converges at the equal to $\lambda_{2}\left(G_{\gamma_{c}}\right)$. We write $\lambda_{i}$ for $\lambda_{i}\left(G_{\gamma_{c}}\right)$ for the sake of notational cumbersomeness. Let $\mathbf{z}_{\mathbf{i}}$ and $\mathbf{y}_{\mathbf{i}}$ be the right and left eigenvectors associated with the eigenvalue $\lambda_{i}$, and let $\lambda_{i}$ in ordered as $\lambda_{1}>\lambda_{2} \geq \cdots$. Then the above argument follows from the eigendecomposition of $G_{\gamma_{c}}$ (assuming that $G_{\gamma_{c}}$ is connected such that $\lambda_{2}\left(G_{\gamma_{c}}\right)<1$, and that $G_{\gamma_{c}}$ is actually eigendecomposable), where the convergence rate is defined precisely as,

$$
\begin{aligned}
r_{B B R} & =\lim _{t \rightarrow \infty} \| G_{\gamma_{c}}^{t}{ }^{-1}{ }^{-}{ }^{\frac{1}{t}} \\
& =\lim _{t \rightarrow \infty}\left\|\left(\mathbf{z}_{\mathbf{1}} \mathbf{y}_{\mathbf{1}}{ }^{\prime-}{ }_{1}-{ }^{-}\right)+\sum_{i=2}^{n} \lambda_{i}^{t} \mathbf{z}_{\mathbf{i}} \mathbf{y}_{\mathbf{i}}{ }^{\prime-} \mathbf{1}\right\|^{\frac{1}{t}} \\
& =\left|\lambda_{2}\right| \lim _{t \rightarrow \infty}\left\|\mathbf{z}_{\mathbf{2}} \mathbf{y}_{\mathbf{2}}{ }^{\prime-} \mathbf{1}+\sum_{i=3}^{n}\left(\frac{\lambda_{i}}{\lambda_{2}}\right)^{t} \mathbf{z}_{\mathbf{i}} \mathbf{y}_{\mathbf{i}}{ }^{\prime-} \mathbf{1}\right\|^{\frac{1}{t}}=\left|\lambda_{2}\right|
\end{aligned}
$$

\subsubsection{Proof of Proposition 14}

First consider the case of Bayesian rational learning mechanism where

$$
U_{i t}\left(a_{i t}^{*}(n), X\right)=-\frac{1}{\delta^{t}} \mathbb{E}_{i, t}\left[\left(\mu_{i, t}(n)-X\right)^{2}\right]=-\frac{1}{\delta^{t}} \operatorname{var}_{i, t}(n)
$$

For a given $\delta$ and $n, U_{i t}\left(a_{i t}^{*}(n), X\right)$ is a non-monotone function of time. The variance $\operatorname{var}_{i, t}(n)$ a generally non-increasing function of time for a given $n$, and $\frac{1}{\delta^{t}}$ is an increasing function of time. Note that the time until the learning process ends under Bayesian ration learning is equal to the diameter $D(G(n))$ of the network. This implies that for a given $\delta$ and $n$, there exists a positive integer $t_{i, e}$ such that at $t=t_{i, e} \leq D(G(n)), U_{i t}\left(a_{i t}^{*}(n), X\right)$ is minimum. The time $t=t_{i, e}$ is the optimal exit time for agent $i$ and it generally depends on $i$ 's position in the network.

The exit time can be ordered with respect to $D(G(n))$ for groups of agents. Let $Q(G(n))$ be a set of agents in $G(n)$ whose optimal exit time is less than the diameter of the graph. That is

$$
Q(G(n))=\left\{i \in N: t_{i, e}<D(G(n))\right\}
$$

and let $\# Q(G(n))$ denote the respective cardinality. Then for two networks $G_{1}(n)$ and $G_{2}(n)$ such that $G_{1}(n)>G_{2}(n)$, then it must be $Q\left(G_{1}(n)\right) \geq Q\left(G_{2}(n)\right)$

Lемма 16: Given a sequence of networks $\{G(n)\}_{n \geq 2}$ and the corresponding sequence $\{Q(G(n))\}_{n \geq 2}$, correct asymptotic learning obtains if and only if

$$
\lim _{n \rightarrow \infty} \# Q(G(n))=0
$$


If condition (5.7.8.2) is not fulfilled then even for a large $n$ there exists a finite number of agents who exit the game after receiving signals only from a finite number of other agents. Lemma 16 can be proved by contradiction.

Consider the contrary case in which $\lim _{n \rightarrow \infty} Q(G(n))=Q_{e}$, that is $Q_{e}$ is a set of agents that exited the game after receiving the private information of a finite number of other agents. Denote by $a_{i, e}$ for the corresponding action taken by an agent $i \in Q_{e}$ at the end of the learning process, and by $n_{i, e}$ as the number of agents whose signals $i$ received before exiting the game. Let $n_{e}=\max _{i \in Q_{e}}\left\{n_{i, e}\right\}$. The above definitions and notations imply that

$$
a_{i, e}(n)=\lim _{n \rightarrow \infty} a_{i, t}(n)=\mu_{i, \infty}(n)
$$

such that the variance of $a_{i, e}(n)$ is

$$
\operatorname{var}\left[a_{i, e}(n)\right] \equiv \operatorname{var}\left[\mu_{i, \infty}(n)\right]
$$

Given the sequence of networks $\{G(n)\}_{n \geq 2}$, then for an agent $i \in Q_{e}$ for whom $n_{i, e}=n_{e}$, we have

$$
\begin{aligned}
\lim _{n \rightarrow \infty} \operatorname{var}\left[a_{i, e}(n)\right] & =\operatorname{var}\left[\mu_{i, \infty}(\infty)\right] \\
& =\operatorname{var}\left[\frac{\sigma^{2}}{n_{e}+\sigma^{2}} \mu_{i, 0}+\frac{1}{n_{e}+\sigma^{2}} \sum_{j=0}^{n_{e}} s_{j}\right] \\
& =\frac{\sigma^{4} \eta^{2}+n_{e} \sigma^{2}}{\left(n_{e}+\sigma^{2}\right)^{2}}
\end{aligned}
$$

From Chernoff bound, it follows that

$$
\begin{aligned}
\lim _{n \rightarrow \infty} \lim _{t \rightarrow \infty} \mathbb{P}\left(\left|a_{i, t}(n)-\bar{\mu}\right|>\epsilon\right) & >\lim _{n \rightarrow \infty} \lim _{t \rightarrow \infty}\left(1-\frac{\operatorname{var}\left[a_{i, t}(n)\right]}{\epsilon}\right) \\
& =1-\frac{\sigma^{4} \eta^{2}+n_{e} \sigma^{2}}{\left(n_{e}+\sigma^{2}\right)^{2}}>0
\end{aligned}
$$

In which case $\lim _{n \rightarrow \infty} \lim _{t \rightarrow \infty} a_{i, t}(n) \neq \bar{\mu}$. If this is true for an agent $i \in Q_{e}$ for whom $n_{i, e}=n_{e}$, and from the fact that for all $i \in Q_{e} n_{i, e} \leq n_{e}$, then (5.7.8.4) must be true for all $i \in Q_{e}$.

Lemma 17: Condition (5.7.8.2) in Lemma 16 is satisfied if and only if for a sequence $\{G(n)\}_{n \geq 2}$ of networks and the corresponding sequence $\{D(G(n))\}_{n \geq 2}$

$$
\lim _{n \rightarrow \infty} \frac{1}{n} D(G(n))=0
$$


The proof of Lemma 17 follows directly from the argument that for a sequence $\{G(n)\}_{n \geq 2}$, if by contradiction the corresponding sequence $\{D(G(n))\}_{n \geq 2}$ is such that $D(G(2))<D(G(2))<\cdots$, then similarly for the sequence $\{Q(G(n))\}_{n \geq 2}$ it must be that $Q(G(2)) \leq Q(G(2)) \leq \cdots$ with at least one strict inequality. In which case condition (5.7.8.2) in Lemma 16 will not be fulfilled.

In the case of bounded-rational learning, the variance of $X$ is constant over time and $n$, which implies that the "discounted" expected loss is non-increasing, unlike the the case of rational learning in which it is non-monotonic in nature. Agents therefore do not have any incentive to wait until longer before taking an action $a \in A$. That is $\lim _{n \rightarrow \infty} \lim _{t \rightarrow \infty} a_{i, t}(n)=\bar{\mu}$ can occur only if the network is complete, such that agents do not have to wait for more than one or two periods before taking an action in $A$. 


\section{VALORIZATION}

This final Chapter of the dissertation discusses the valorization opportunities that this $\mathrm{PhD}$ thesis presents. This is in accordance with article 23.5 of the Regulation governing the attainment of doctoral degrees at Maastricht University. I start by providing the summary of the socio-scientific implications of the thesis findings. The social and economic relevance of the study will be followed with a description of the potential target groups for whom the results may be of interest. I will then present the degree of innovativeness of the research methods used and how the results of the study will be disseminated to a wider audience.

This dissertation has focused on three aspects of social and economic interactions: coordinated change in strategic behavior, opinion formation and the nature of public beliefs, and the role of the structure of social interactions in shaping both aspects.

The analysis and results of the first part of the project can be directly applied to the scientific study of how social institutions and technologies may arise and evolve. Social institutions and technologies are dynamically similar in that they both require coordinated change in expectations and behavior of individuals. Our analysis of coordinated change in strategic behavior therefore directly extends to these specific cases of social and economic interactions. Social institutions can be formally defined as mechanisms that facilitate interactions and coordination among individuals of any given society. Examples of social institutions include social norms, forms of corporate governance, terms of economic contracts and private property rights. Social technologies in this context refer to technological products with positive returns to adoption, such as information technologies. This dissertation thus contributes to our understanding of how such institutions and technologies evolve. And in particular, it shows how innovations can bring about change in social institutions and technologies.

This thesis finds that $(a)$ the payoff gains from the potentially new social institutional or technology and $(b)$ the structure of day-to-day social interactions, matter in 
shaping their evolution. These two factors determine whether or not the new institution and technology can gain a foothold in the society. Our study shows that for societies in which connections between individuals are sparse, incremental changes are feasible. By contrast, for societies in which individuals are highly connected, only radical changes are feasible. These findings are particularly relevant in the current Information and Social Ages in which societal interactions are shaped by digital technologies because social interactions such as online relationships, collaboration and information sharing are becoming more and more structured.

Beyond the above mentioned socio-scientific relevance, the findings in this thesis are also of relevance to firms and policy makers. Social norms and interactions influence productivity and organizational efficiency in corporations and public organizations. Norms define expected behavior and hence accepted levels of performance by members of an organization. Our findings then imply that when thinking about changing the working norms within an organization, not only does one have to consider the potential reward from the desired norm but also the structure of interactions among the members of the organization. Perhaps most importantly, the results in Chapter 3 imply that if the structure of interactions is known, then the design of rewards to be offered for adopting a new norm can be determined.

In relation to firms as target groups, our findings can directly be applied in designing market expansionary strategies. The thesis theoretically shows that if the preferences over a set of consumer or technological products exhibit network externalities, then for a given structure of social interactions among consumers, one can determine the level of innovation on a product required for it to takeover (at least the largest share of) the market. Examples of consumer products that exhibit network externalities range from durable goods in which buyers care about post-purchase services to IT products in which its benefits depend on how many others in one's social network adopt it. In the case where two competing products with network externalities co-exist within the market, our findings imply that having knowledge of the network of interactions among consumers enables determination of the level of incremental innovation on the product for it to gain a larger share of the market. Similarly, when a new product is introduced to the market, it is not only the relative gains that the new product offers to the consumers but also the structure of interactions among consumers that determines its success.

Our results are also of relevance to policy makers. The success of any economic policy depends on public opinion towards it. Consider an example of the current debate on the exact cause of climate change. After examining nearly 12,000 peerreviewed scientific papers, Cook et al. [29] found that $97.1 \%$ of them endorsed the 
consensus position that humans are causing global warming. By contrast, only $41 \%$ of the American public says that global warming is anthropogenic. The success of any economic policy aiming at reducing anthropogenic factors such as $\mathrm{Co}$ emissions will then depend on how it affects the $59 \%$ of the population with an alternative opinion on causes of global warming. The second part (Chapter 5) of this thesis studies conditions under which public consensus and disagreement may arise and how fast structured societies converge to stable public opinion. We show how historical factors (prior beliefs), the manner in which individuals assimilate new information and networks governing information exchange interactively shape public opinion. Knowledge of these factors can be used by policy makers to promote desirable economic policies.

In terms of innovativeness, this thesis introduces novel analytical methods that are not limited to the field of social sciences. The methods introduced include probability techniques for studying dynamic interaction systems that involve experimentation and communication among interacting entities. It also introduces theoretical methods for analyzing processes in which interactions among entities are decentralized. Such interaction systems can be found in the field of biological sciences and computer science. Our theoretical methods can for example be employed to study flocking and herding behavior in social animals, or biological evolutionary processes. Similarly, they can also be applied to the study of consensus and coordination in sensor and electric networks. The theoretical methods introduced will therefore be of use to a wider scientific circle.

Finally, the dissemination of the outcomes of this project is already in progress. The results have been presented to international audiences in conferences and seminars. Presentations have been given at The Centre d'Economie de La Sorbonne and Paris School of Economics, University of Cergy Pontoise THEMA, Cournot seminars at Bureau d'Economie Théorique et Appliquée (BETA), University of Strasbourg, Royal Economic Society Postgraduate Presentation Meeting \& Job Market, 1oth Workshop on Networks in Economics and Sociology at Utrecht School of Economics, and Maastricht Lecture Series in Economics. The four main chapters of the thesis have been rewritten into journal article formats, all of which are under submission for publication in top peer-reviewed economic journals. 

[1] Acemoglu, D., Chernozhukov, V., and Yildiz, M. (2009). Fragility of asymptotic agreement under bayesian learning. Technical report.

[2] Acemoglu, D., Dahleh, M. A., Lobel, I., and Ozdaglar, A. (2011). Bayesian learning in social networks. The Review of Economic Studies, 78(4):1201-1236.

[3] Aldous, D. (1987). On the markov chain simulation method for uniform combinatorial distributions and simulated annealing. Probability in the Engineering and Informational Sciences, 1(O1):33-46.

[4] Aldous, D. and Fill, J. A. (1994). Reversible markov chains.

[5] Alon, N. (1986). Eigenvalues and expanders. Combinatorica, 6(2):83-96.

[6] Alon, N. and Milman, V. D. (1985). $\lambda_{1}$, isoperimetric inequalities for graphs, and superconcentrators. Journal of Combinatorial Theory, Series B, 38(1):73- 88.

[7] Alós-Ferrer, C. and Netzer, N. (2010). The logit-response dynamics. Games and Economic Behavior, 68(2):413-427.

[8] Alós-Ferrer, C. and Weidenholzer, S. (2007). Partial bandwagon effects and local interactions. Games and Economic Behavior, 61(2):179-197.

[9] Alós-Ferrer, C. and Weidenholzer, S. (2008). Contagion and efficiency. Journal of Economic Theory, 143(1):251-274.

[10] Anderson, S. P., de Palma, A., and Thisse, J.-F. (1992). Discrete Choice Theory of Product Differentiation. The MIT Press.

[11] Asavathiratham, C. (2001). The influence model: a tractable representation for the dynamics of networked Markov chains. Massachusetts Institute of Technology, Dept. of Electrical Engineering and Computer Science, Ph.D Thesis.

[12] Bala, V. and Goyal, S. (1998). Learning from neighbours. Review of Economic Studies, 65(3):595-621.

[13] Banerjee, A. V. (1992). A simple model of herd behavior. The Quarterly Journal of Economics, 107(3):797-817. 
[14] Beggs, A. (2005). Waiting times and equilibrium selection. Economic Theory, 25(3):599-628.

[15] Bergin, J. and Lipman, B. L. (1996). Evolution with state-dependent mutations. Econometrica, 64(4):943-56.

[16] Bikhchandani, S., Hirshleifer, D., and Welch, I. (1992). A theory of fads, fashion, custom, and cultural change as informational cascades. Journal of Political Economy, 100(5):992-1026.

[17] Billings, L. and Schwartz, I. B. (2008). Identifying almost invariant sets in stochastic dynamical systems. Chaos: An Interdisciplinary Journal of Nonlinear Science, 18(2):023122.

[18] Binmore, K., Samuelson, L., and Young, P. (2003). Equilibrium selection in bargaining models. Games and Economic Behavior, 45(2):296-328.

[19] Blume, L. E. (1995). The statistical mechanics of best-response strategy revision. Games and Economic Behavior, 11(2):111-145.

[20] Blume, L. E. (2003). How noise matters. Games and Economic Behavior, 44(2):251271.

[21] Bollobás, B. (1981). The diameter of random graphs. Transactions of the American Mathematical Society, 267(1):41-52.

[22] Bonacich, P. and Lloyd, P. (2001). Eigenvector-like measures of centrality for asymmetric relations. Social Networks, 23(3):191-201.

[23] Bremaud, P. (1999). Markov chains: Gibbs fields, Monte Carlo simulation, and queues, volume 31. springer.

[24] Brown, G. W. (1951). Iterative solution of games by fictitious play. Activity analysis of production and allocation, 13(1):374-376.

[25] Camerer, C. and Ho, T.-H. (1999). Experience-weighted Attraction Learning in Normal Form Games. Econometrica, 67(4):827-874.

[26] Catoni, O. (1999). Simulated annealing algorithms and markov chains with rare transitions. In Azéma, J., Émery, M., Ledoux, M., and Yor, M., editors, Séminaire de probabilités XXXIII, pages 69-119. Springer, Berlin Heidelberg.

[27] Chamley, C. and Gale, D. (1994). Information revelation and strategic delay in a model of investment. Econometrica, 62(5):1065-85. 
[28] Chung, F. R. (1989). Diameters and eigenvalues. Journal of the American Mathematical Society, pages 187-196.

[29] Cook, J., Nuccitelli, D., Green, S. A., Richardson, M., Winkler, B., Painting, R., Way, R., Jacobs, P., and Skuce, A. (2013). Quantifying the consensus on anthropogenic global warming in the scientific literature. Environmental Research Letters, 8(2):024024.

[30] Cooper, D. J., Garvin, S., and Kagel, J. H. (1997). Signalling and Adaptive Learning in an Entry Limit Pricing Game. RAND Journal of Economics, 28(4):662-683.

[31] Cripps, M. W., Ely, J. C., Mailath, G. J., and Samuelson, L. (2008). Common learning. Econometrica, 76(4):909-933.

[32] Demarzo, P. M., Vayanos, D., and Zwiebel, J. (2003). Persuasion bias, social influence, and unidimensional opinions. The Quarterly Journal of Economics, 118(3):909968.

[33] Diaconis, P. and Stroock, D. (1991). Geometric bounds for eigenvalues of markov chains. The Annals of Applied Probability, pages 36-61.

[34] Dixit, A. K. and Weibull, J. W. (2007). Political polarization. Proceedings of the National Academy of Sciences, 104(18):7351-7356.

[35] Dokumac1, E. and Sandholm, W. H. (2011). Large deviations and multinomial probit choice. Journal of Economic Theory, 146(5):2151-2158.

[36] Durrett, R. (2006). Random Graph Dynamics. Cambridge Series on Statistical and Probabilistic Mathematics. Cambridge University Press.

[37] Ellison, G. (1993). Learning, local interaction, and coordination. Econometrica, 61(5):1047-1071.

[38] Ellison, G. (2000). Basins of attraction, long-run stochastic stability, and the speed of step-by-step evolution. Review of Economic Studies, 67(1):17-45.

[39] Erev, I. and Roth, A. E. (1998). Predicting How People Play Games: Reinforcement Learning in Experimental Games with Unique, Mixed Strategy Equilibria. American Economic Review, 88(4):848-81.

[40] Fiedler, M. (1973). Algebraic connectivity of graphs. Czechoslovak Mathematical Journal, 23(98):298-305. 
[41] Foster, D. and Young, P. (1990). Stochastic evolutionary game dynamics. Theoret. Population Biol.

[42] Freidlin, M. and Wentzell, A. D. (1984). Random perturbations of dynamical systems. New York: Springer Verlag.

[43] Froyland, G. (2005). Statistically optimal almost-invariant sets. Physica D: Nonlinear Phenomena, 200(3):205-219.

[44] Fudenberg, D. and Kreps, D. M. (1993). Learning mixed equilibria. Games and Economic Behavior, 5(3):320-367.

[45] Fudenberg, D. and Kreps, D. M. (1995). Learning in extensive-form games i. self-confirming equilibria. Games and Economic Behavior, 8(1):20-55.

[46] Gale, D. and Kariv, S. (2003). Bayesian learning in social networks. Games and Economic Behavior, 45(2):329-346.

[47] Geanakoplos, J. D. and Polemarchakis, H. M. (1982). We can't disagree forever. Journal of Economic Theory, 28(1):192 - 200.

[48] Goeree, J. K. and Holt, C. A. (1999). Stochastic game theory: For playing games, not just for doing theory. Proceedings of the National Academy of Sciences, 96(19):10564-10567.

[49] Golub, B. and Jackson, M. O. (2010). Naïve learning in social networks and the wisdom of crowds. American Economic Journal: Microeconomics, 2(1):112-49.

[50] Hofbauer, J. and Sandholm, W. H. (2002). On the global convergence of stochastic fictitious play. Econometrica, 70(6):2265-2294.

[51] Hofbauer, J. and Sandholm, W. H. (2007). Evolution in games with randomly disturbed payoffs. Journal of Economic Theory, 132(1):47-69.

[52] Horn, R. A. and Johnson, C. R. (1990). Matrix Analysis. Cambridge University Press.

[53] Huck, S., Kübler, D., and Weibull, J. (2012). Social norms and economic incentives in firms. Journal of Economic Behavior \& Organization, 83(2):173 - 185.

[54] Jackson, M. O. (2008). Social and Economic Networks. Princeton University Press. Princeton University Press.

[55] Jackson, M. O. and Yariv, L. (2007). Diffusion of Behavior and Equilibrium Properties in Network Games. American Economic Review, 97(2):92-98. 
[56] Kalai, E. and Lehrer, E. (1993). Subjective equilibrium in repeated games. Econometrica: Journal of the Econometric Society, pages 1231-1240.

[57] Kandori, M., Mailath, G. J., and Rob, R. (1993). Learning, mutation, and long run equilibria in games. Econometrica, 61(1):29-56.

[58] Kandori, M. and Rob, R. (1998). Bandwagon effects and long run technology choice. Games and Economic Behavior, 22(1):30-60.

[59] Kim, C. and Wong, K.-C. (2010). Long-run equilibria with dominated strategies. Games and Economic Behavior, 68(1):242-254.

[6o] Kirman, A. P. and Vriend, N. J. (2001). Evolving market structure: An ace model of price dispersion and loyalty. Journal of Economic Dynamics and Control, 25(3):459502.

[61] König, M., Tessone, C. J., and Zenou, Y. (2009). A dynamic model of network formation with strategic interactions. Technical report.

[62] Krasucki, P. (1996). Protocols forcing consensus. Journal of Economic Theory, 7O(1):266-272.

[63] Kreindler, G. E. and Young, H. P. (2013). Fast convergence in evolutionary equilibrium selection. Games and Economic Behavior, 80:39-67.

[64] Kreindler, G. E. and Young, H. P. (2014). Rapid innovation diffusion in social networks. Proceedings of the National Academy of Sciences, 111 (Supplement 3):1088110888.

[65] Kuran, T. and Sandholm, W. H. (2008). Cultural integration and its discontents. The Review of Economic Studies, 75(1):201-228.

[66] Lee, I. H., Szeidl, A., and Valentinyi, A. (2003). Contagion and state dependent mutations. The B.E. Journal of Theoretical Economics, 3(1):1-29.

[67] Lee, I. H. and Valentinyi, A. (2000). Noisy contagion without mutation. Review of Economic Studies, 67(1):47-56.

[68] Lelarge, M. (2012). Diffusion and cascading behavior in random networks. Games and Economic Behavior, 75(2):752-775.

[69] Lettau, M. and Uhlig, H. (1999). Rules of thumb versus dynamic programming. American Economic Review, 89:148-174. 
[70] Levin, D. A., Peres, Y., and Wilmer, E. L. (2009). Markov chains and mixing times. American Mathematical Society.

[71] Maruta, T. (1997a). On the Relationship between Risk-Dominance and Stochastic Stability. Games and Economic Behavior, 19(2):221-234.

[72] Maruta, T. (1997b). On the relationship between risk-dominance and stochastic stability. Games and Economic Behavior, 19(2):221 - 234.

[73] McKelvey, R. D. and Palfrey, T. R. (1992). An Experimental Study of the Centipede Game. Econometrica, 6o(4):803-36.

[74] Michihiro, K. and Rob, R. (1998). Bandwagon effects and long run technology choice. Games and Economic Behavior, 22(1):30-60.

[75] Mitra, P. (2009). Entrywise bounds for eigenvectors of random graphs. The Electronic Journal of Combinatorics, 16(1):Research Paper R131, 18 p.

[76] Mohar, B. (1989). Isoperimetric numbers of graphs. Journal of Combinatorial Theory, Series B, 47(3):274-291.

[77] Montanari, A. and Saberi, A. (2010). The spread of innovations in social networks. Proceedings of the National Academy of Sciences.

[78] Morris, S. (2000). Contagion. Review of Economic Studies, 67(1):57-78.

[79] Morris, S., Rob, R., and Shin, H. S. (1995). Dominance and Belief Potential. Econometrica, 63(1):145-57.

[8o] Mueller-Frank, M. (2013). A general framework for rational learning in social networks. Theoretical Economics, 8(1):1-40.

[81] Murto, P. and Välimäki, J. (2011). Learning and Information Aggregation in an Exit Game. Review of Economic Studies, 78(4):1426-1461.

[82] Myatt, D. P. and Wallace, C. (2003). A multinomial probit model of stochastic evolution. Journal of Economic Theory, 113(2):286-301.

[83] Nachbar, J. H. (2005). Beliefs in repeated games. Econometrica, 73(2):459-480.

[84] Nasar, S. (1998). A Beautiful Mind. Simon and Schuster, New York.

[85] Nash, J. (1960). Non-cooperative Games. PhD thesis, Princeton University. 
[86] Newman, M. E., Watts, D. J., and Strogatz, S. H. (2002). Random graph models of social networks. Proceedings of the National Academy of Sciences, 99(suppl 1):25662572.

[87] Newman, M. E. J. (2003). The structure and function of complex networks. SIAM Review, 45(2):167-256.

[88] Nöldeke, G. and Samuelson, L. (1997). A dynamic model of equilibrium selection in signaling markets. Journal of Economic Theory, 73(1):118-156.

[89] Okada, D. and Tercieux, O. (2012). Log-linear dynamics and local potential. Journal of Economic Theory, 147(3):1140-1164.

[90] Opolot, D. and Azomahou, T. (2014a). Beliefs dynamics in communication networks. Technical report.

[91] Opolot, D. and Azomahou, T. (2014b). Epsilon-stability and the speed of learning in network games. Technical report.

[92] Parikh, R. and Krasucki, P. (1990). Communication, consensus, and knowledge. Journal of Economic Theory, 52(1):178-189.

[93] Robinson, J. (1951). An iterative method of solving a game. Annals of mathematics, pages 296-301.

[94] Robson, A. J. and Vega-Redondo, F. (1996). Efficient Equilibrium Selection in Evolutionary Games with Random Matching. Journal of Economic Theory, 70(1):6592.

[95] Rosenberg, D., Solan, E., and Vieille, N. (2009). Informational externalities and emergence of consensus. Games and Economic Behavior, 66(2):979-994.

[96] Roth, A. E. and Erev, I. (1995). Learning in extensive-form games: Experimental data and simple dynamic models in the intermediate term. Games and economic behavior, 8(1):164-212.

[97] Ryan, B. and Gross, N. (1943). The diffusion of hybrid seed corn in two Iowa communities. Rural Sociology, 8(1):15-24.

[98] Sandholm, W. (2010). Population Games and Evolutionary Dynamics. Economic Learning and Social Evolution. MIT Press.

[99] Sandholm, W. H. (2001). Almost global convergence to p-dominant equilibrium. International Journal of Game Theory, pages 107-116. 
[10o] Sandholm, W. H., Tercieux, O., and Oyama, D. (2014). Sampling best response dynamics and deterministic equilibrium selection. Theoretical Economics.

[101] Seneta, E. (1979). Coefficients of ergodicity: Structure and applications. Advances in Applied Probability, 11(3):576-590.

[102] Seneta, E. (1993). Non-negative matrices. New York: Wiley.

[103] Sethi, R. and Yildiz, M. (2012). Public disagreement. American Economic Journal: Microeconomics, 4(3):57-95.

[104] Sethi, R. and Yildiz, M. (2013). Perspectives, opinions, and information flows. Working paper, Columbia University and the Santa Fe Institute.

[105] Shapley, L. S. et al. (1964). Some topics in two-person games. Advances in game theory, 52:1-29.

[106] Simon, H. and Ando, A. (1961). Aggregation of variables in dynamic systems. Econometrica, 29(2):111-138.

[107] Sinclair, A. (1992). Improved bounds for mixing rates of markov chains and multicommodity flow. Combinatorics, Probability and Computing, 1(04):351-370.

[108] Sinclair, A. and Jerrum, M. (1989). Approximate counting, uniform generation and rapidly mixing markov chains. Information and Computation, 82(1):93-133.

[109] Smith, L. and Sorensen, P. (2000). Pathological outcomes of observational learning. Econometrica, 68(2):371-398.

[110] Takacs, C. (2006). On the fundamental matrix of finite state markov chains, its eigensystem and its relation to hitting times. Mathematica Pannonica, 183:193.

[111] Vega-Redondo, F. (1997). The evolution of walrasian behavior. Econometrica, $65(2): 375-384$.

[112] Wolfowitz, J. (1963). Products of indecomposable, aperiodic, stochastic matrices. Proceedings of the American Mathematical Society, 14(5):733-737.

[113] Young, H. P. (1993). The evolution of conventions. Econometrica, 61(1):57-84.

[114] Young, H. P. (2009). Innovation diffusion in heterogeneous populations: Contagion, social influence, and social learning. The American economic review, pages 1899-1924. 
[115] Young, P. H. (2011a). The dynamics of social innovation. Proceedings of the National Academy of Sciences, 108(4):21285-21291.

[116] Young, P. H. (2011b). The dynamics of social innovation. Proceedings of the National Academy of Sciences, 108(Supplement 4):21285-21291. 

UNITED NATIONS UNIVERSITY

\section{UNU-MERIT}

2015

\section{Daniel C. Opolot}

The evolution of beliefs and strategic behavior

\section{Alejandro Lavopa}

Structural Transformation and Economic Development: Can Development Traps be Avoided?

\section{Jinjin Zhao}

Urban water management reform: The case of China

\section{4}

\section{Dirk Crass}

The Impact of Brands on Innovation and Firm Performance: Empirical Evidence from Germany

\section{Samyukta Bhupatiraju}

The Geographic Dimensions of Growth and Development

\section{François Lafond}

The evolution of knowledge systems

\section{Annalisa Primi}

Promoting Innovation in Latin America: What Countries Have Learned (and What They Have Not) in Designing and Implementing Innovation and Intellectual Property Policies

\section{Fatoumata Lamarana Diallo}

Evaluation of Meal and Deworming Programs for Primary Schools in Rural Senegal

\section{3}

\section{Anant Kamath}

Information Sharing through Informal Interaction in Low-Tech Clusters

\section{Flavia Pereira de Carvalho}

What we talk about when we talk about Brazilian Mulitantionals: an investigation on Brazilian FDI, economic structure, innovation and the relationship between them

\section{Jun Hou}

Complementarity in Innovation and Development: A Cross-country

Comparison

\section{Rufin Baghana}

Impacts of Government Incentives to R\&D, Innovation and Productivity:

A Microeconometric Analysis of the Québec Case

\section{Lilia I. Stubrin}

High-Tech Activities in Emerging Countries: A Network perspective on the Argentinean biotech activity

\section{2}

\section{Abdul Waheed}

Innovation Determinants

and Innovation as a Determinant:

Evidence from Developing Countries

\section{Bilal Mirza}

Energy Poverty and Rural Energy Markets in Pakistan 


\section{Benjamin Engelstätter}

Enterprise Software and Video Games: An Empirical Analysis

\section{Fulvia Farinelli}

Natural Resources, Innovation and Export Growth: The Wine Industry in Chili and Argentina

\section{Rodolfo Lauterbach}

Innovation in Manufacturing: From Product Variety and Labor Productivity Growth to Economic Development in Chile

\section{Kirsten Wiebe}

Quantitative Assessment of Sustainable Development and Growth in Sub-Saharan Africa.

\section{Julio Miguel Rosa}

Organizational Strategies, Firms' Performance and Spatial Spillovers. The Canadian Case in Research and Development.

\section{Johannes Wilhelmus Marie Boels} Joseph Schumpeter, honderd jaar economische ontwikkeling. Een historischtheoretische beschouwing.

\section{1}

\section{Daniel Vertesy}

Interrupted Innovation: Emerging economies in the structure of the global aerospace industry.

\section{Tina Saebi}

Successfully managing alliance portfolios: an alliance capability view.

\section{Nora Engel}

Tuberculosis in India - A case of innovation and control.

\section{Evans Mupela}

Connectivity and growth in Sub-Saharan Africa: The role of communication satellites

\section{Nantawan Kwanjai}

Cross cultural intelligence amid intricate cultural webs: A tale of the UnDutchables in the land of 1002 smiles

\section{Lina Sonne}

Innovation in Finance to Finance Innovation: Supporting pro-poor entrepreneur-based innovation

\section{0}

\section{Fernando Santiago}

Human Resources Management Practices and Learning for Innovation in Developing Countries: Pharmaceutical Firms in Mexico

\section{Zakaria Babutsidze}

Essays on Economies with Heterogenous Interacting Consumers

\section{Bertha Vallejo}

Learning and Innovation Under Changing Market Conditions: The Auto Parts Industry in Mexico

\section{Donatus Ayitey}

Technical Change, Competitiveness and Poverty Reduction: A Study of the Ghanaian Apparel Industry

\section{Sergey Fillipov}

Multinational Subsidiary Evolution: Corporate Change in New EU Member States 


\section{Asel Doranova}

Technology Transfer and Learning under the Kyoto regime; Exploring the

Technological Impact of CDM projects in developing countries

\section{9}

\section{Alexis Habiyaremye}

From Primary Commodity Dependence to Diversification and Growth". "Absorptive Capacity and Technological Catch Up in Botswana and Mauritius".

\section{Yoseph Getachew}

The Role of Public Capital in Economic Development

\section{Sandra Leitner}

Embodied Technological Change and Patterns of Investment in Austrian Manufacturing

\section{Semih Akçomak}

The Impact of Social Capital on Economic and Social Outcomes

\section{Abraham Garcia}

The Role of Demand in Technical Change

\section{Saurabh Arora}

Coherence in socio-technical systems: a network perspective on the innovation process

\section{8}

\section{Rutger Daems}

Medicines for the developing world

\section{Johannes Hanel}

Assessing Induced Technology: Sombart's Understanding of Technical Change in the History of Economics

\section{Rifka Weehuizen}

Mental Capital: the economic significance of mental health

\section{Danielle Cloodt}

The relationship between R\&D partnership formation, social embeddedness and innovative performance

\section{Sabine Fuss}

Sustainable Energy Development under Uncertainty

\section{7}

\section{Tobias Kronenberg}

Reconciling Environmental Conservation with Economic Prosperity: The Feasibility of Double Dividends in the Short and Long Run

\section{Viktoria Kravtsova}

Assessing the Impact of Foreign Direct Investment in Transition Economies

\section{Suhail Sultan}

The Competitive Advantage of Small and Medium Sized Enterprises: The Case of Jordan's Natural Stone Industry

\section{6}

\section{Bulat Sanditov}

Essays on Social Learning and Imitation

\section{Mamata Parhi}

Dynamics of New Technology Diffusion: A Study of the Indian Automotive Industry

\section{Andreas Reinstaller}

Social structures and the innovation process: Their role in the demand of firms and consumers 


\section{Rose Kiggundu}

Innovation systems and development: the journey of a Beleaguered Nile Perch

Fishery in Uganda

\section{Thomas Pogue}

The Evolution of Research Collaboration in South African Gold Mining: 1886-1933

\section{Geoffrey Gachino}

Foreign Direct Investment, Spillovers and Innovation: The Case of Kenyan

Manufacturing Industry

\section{0. Önder Nomaler}

Technological Change, International Trade and Growth: An Evolutionary, Multi-

Agents-Based Modeling Approach

\section{5}

39. Samia Satti Osman Mohamed-Nour Change and Skill Development in the Arab Gulf Countries

\section{Elad Harison}

Intellectual Property Rights: Economics and Policy Analysis

\section{Daniel Dalohoun}

The relationship between R\&D partnership formation, social embeddedness and innovative performance: a multi-level approach of social embeddedness

\section{Müge Ozman}

Networks, Organizations and Knowledge

\section{Bas Straathof}

Product variety and economic growth: The counteracting effects of scale and idiosyncrasy

\section{Wilfred Schoenmakers}

Knowledge Flows between Multinational Companies: A Patent Data Analysis

\section{Myriam Cloodt}

Mergers and Acquisitions (M\&As) in HighTech Industries: Measuring the Post-M\&A Innovative Performance of Companies

\section{4}

\section{Paola Criscuolo}

R\&D Internationalisation and Knowledge Transfer. Impact on MNEs and their Home Countries

\section{Maarten Verkerk}

Trust and Power on the Shop Floor

\section{Gottfried Leibbrandt}

Adoption, harmonization and succession of network technologies across countries

\section{Mark Sanders}

Skill Biased Technical change - Its Origins, the Interaction with the Labour Market and Policy Implications

\section{3}

\section{Nadine Roijakkers}

Inter-firm cooperation in high-tech industries: a study of R\&D partnerships in pharmaceutical biotechnology

\section{Viki Sonntag}

Speed, Scale and Sustainability

\section{Masaru Yarime}

From End-of-Pipe Technology to Clean Technology 


\section{Stéphane Malo}

The combinatorial Chemistry Revolution Sustaining a Superior Performance

Position through Technological Learning

\section{2}

\section{Annelies Hogenbirk}

Determinants of Inward Foreign Direct

Investment: the Case of the Netherlands

\section{1}

23. John Adeoti

Technology Investment in Pollution

Control in Sub-Saharan Africa: The Case of the Nigerian Manufacturing Industry

\section{Edward Huizenga}

Innovation Management: How

Frontrunners Stay Ahead. An Empirical

Study on Key Success Factors in the ICT

sector

\section{0}

\section{Machiel van Dijk}

Technological Change and the Dynamics of Industries. Theoretical Issues and Empirical evidence from Dutch Manufacturing

\section{9}

\section{Jan Cobbenhagen}

Managing Innovation at the Company Level: A Study on Non-Sector-Specific Success Factors

\section{Marjolein Caniëls}

Regional Growth Differentials: The Impact of Locally Bounded Knowledge Spillovers

\section{8}

\section{Aldo Geuna}

Resource allocation and knowledge production: Studies in the economics of university research

\section{6}

\section{Reinoud Joosten}

Dynamics, Equilibria, and Values

\section{Hugo Kruiniger}

Investment, R\&D, and the Financing

Decisions of the Firm

\section{5}

\section{Hans van Meijl}

Endogenous Technological Change: The Case of Information Technology.

Theoretical Considerations and Empirical Results

\section{René Kemp}

Environmental Policy and Technical

Change. A Comparison of the

Technological Impact of Policy Instruments

\section{Rohini Acharya}

The Impact of New Technologies on Economic Growth and Trade. A Case Study of Biotechnology

\section{Geert Duysters}

The Evolution of Complex Industrial Systems. The Dynamics of Major IT Sectors

\section{Marjan Groen}

Technology, Work and Organisation, A Study of the Nursing Process in Intensive Care Units 
10. Huub Meijers

On the Diffusion of Technologies in a Vintage Framework; Theoretical

Considerations and Empirical Results

\section{Theon van Dijk}

The Limits of Patent Protection. Essays on the Economics of Intellectual Property

Rights

\section{Hans Voordijk}

Naar Integrale Logistiek in Bedrijfsketens, Ontwikkelingen in de Bouw

\section{3}

\section{Paul Diederen}

Technological Progress in Enterprises and Diffusion of Innovations. Theoretical

Reflections and Empirical Evidence.

\section{Ben Dankbaar}

Economic Crisis and Institutional Change. The crisis of Fordism from the perspective of the automobile industry

\section{Hanno Roberts}

Accountability and Responsibility: The Influence of Organisation Design on

Management Accounting

\section{2}

\section{Bart Verspagen}

Uneven Growth Between Interdependent Economies. An Evolutionary View on Technology Gaps, Trade and Growth

\section{Sjoerd Romme}

A Self-organization Perspective on Strategy Formation

\section{John Spangenberg}

Economies of Scale, and Atmosphere in Research Organisations

\section{8}

\section{John Hagedoorn}

Evolutionary and heterodox innovation analysis: a study of industrial and technological development in process control and information technology 


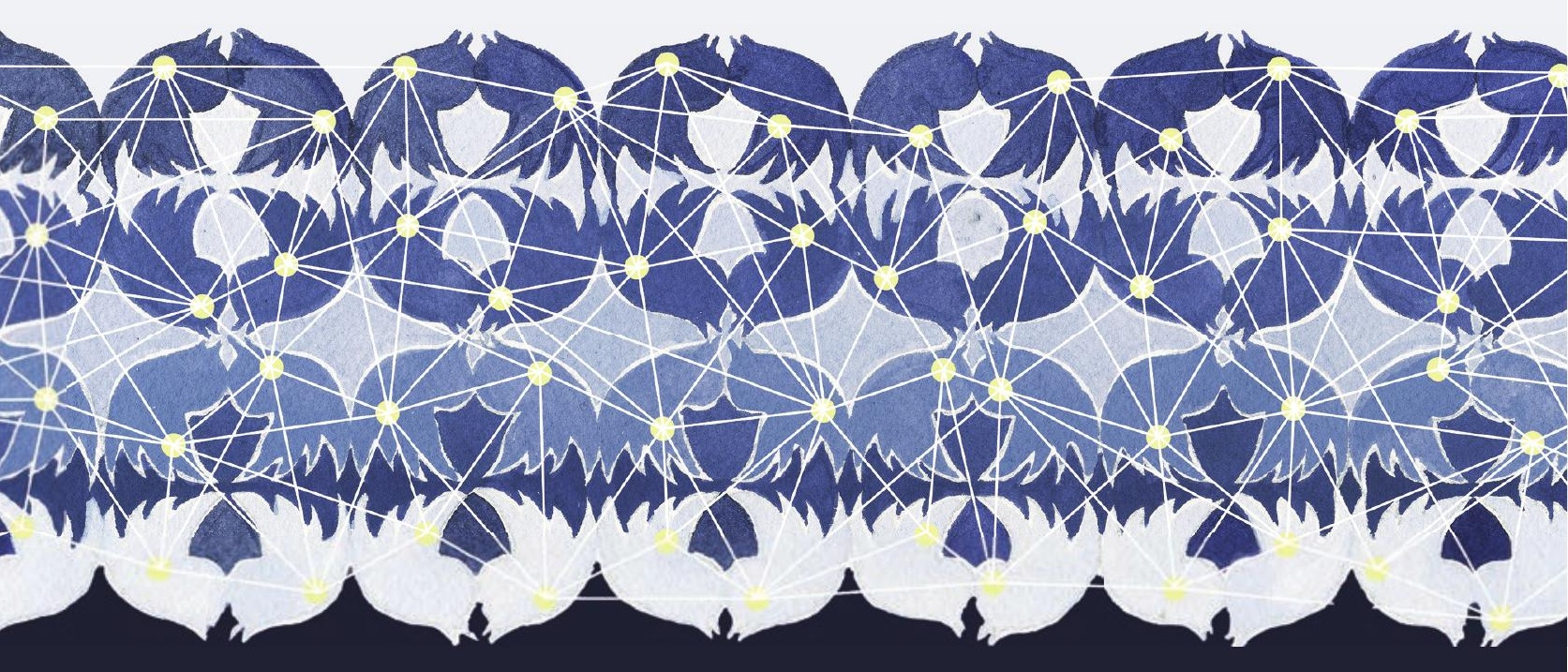

